University of Tennessee Health Science Center UTHSC Digital Commons

\title{
Diffusion Tensor Imaging in Pediatric Brain Tumor Patients
}

Nicholas S. Phillips

University of Tennessee Health Science Center

Follow this and additional works at: https://dc.uthsc.edu/dissertations

Part of the Diagnosis Commons

\section{Recommended Citation}

Phillips, Nicholas S. , "Diffusion Tensor Imaging in Pediatric Brain Tumor Patients" (2009). Theses and Dissertations (ETD). Paper 201. http://dx.doi.org/10.21007/etd.cghs.2009.0244.

This Dissertation is brought to you for free and open access by the College of Graduate Health Sciences at UTHSC Digital Commons. It has been accepted for inclusion in Theses and Dissertations (ETD) by an authorized administrator of UTHSC Digital Commons. For more information, please contact jwelch30@uthsc.edu. 


\title{
Diffusion Tensor Imaging in Pediatric Brain Tumor Patients
}

\author{
Abstract \\ In this dissertation, we outline our efforts to introduce an advanced MRI imaging technique called \\ Diffusion Tensor Imaging (DTI) to the pediatric brain tumor population. We discuss the theory and \\ application of DTI as it was performed in a series of translational investigations at St. Jude Children's \\ Research Hospital. We present evidence of how the introduction of this technique impacted diagnosis, \\ and treatment. And finally, we demonstrate how DTI was used to investigate cognitive morbidities \\ associated with cancer treatment and how this research provided insight into the underlying \\ pathophysiology involved in the development of these treatment sequela. \\ This research has generated important insights into the fundamental causes of neuroanatomical and \\ cognitive deficits associated with cancer and cancer therapy. The use of DTI has permitted us to identify \\ potential targets for improved radiological and surgical techniques as well as targets for pharmacological \\ and behavioral interventions that might improve cognitive function in cancer survivors. The discoveries \\ here afford us an opportunity to reduce the negative effects of cancer therapy on patients treated in the \\ future while maintaining successful survival rates.

\section{Document Type} \\ Dissertation \\ Degree Name \\ Doctor of Philosophy (PhD)

\section{Program} \\ Biomedical Engineering and Imaging \\ Research Advisor \\ Robert J. Ogg Ph.D.

\section{Keywords} \\ DTI, imaging, pediatrics, brain tumors, cancer, MRI

\section{Subject Categories} \\ Analytical, Diagnostic and Therapeutic Techniques and Equipment | Diagnosis | Medicine and Health \\ Sciences

\section{Comments} \\ Six month campus-only embargo expired December 2009
}




\title{
Diffusion Tensor Imaging in Pediatric Brain Tumor Patients
}

\author{
A Dissertation \\ Presented for \\ The Graduate Studies Council \\ The University of Tennessee \\ Health Science Center
}

\author{
In Partial Fulfillment \\ Of the Requirements for the Degree \\ Doctor of Philosophy \\ In the Joint Graduate Program in Biomedical Engineering and Imaging \\ From The University of Tennessee \\ and \\ The University of Memphis
}

By

Nicholas S. Phillips

May 2009 
Chapter 3 (C) 2003 by American Journal of Neuroradiology. Chapter 4 (C) 2008 by Journal of Neurosurgery Pediatrics.

Chapter 5 (C) by 2005 Journal of Neurosurgery. All other material (C) 2009 by Nicholas S Phillips. 


\section{Dedication}

This dissertation is dedicated to my wife, Carolyn Phillips, who has the faith and patience of a saint. 


\section{Acknowledgments}

I thank my wife, Carolyn Phillips, for her love and support. I wish to thank my advisor Dr. Rob Ogg for his guidance and friendship. I also appreciate the support of my committee. To Dr. Waleed Gaber, I wish to thank him for his help and guidance in unraveling the mysteries of the mathematics of modern physics. Dr. Gene Reddick, I wish to thank for his continous support, reassurances and collaboration. Dr. Kathleen Helton, I wish to thank for her early vision and creating opportunities for us to move our work from the "bench to the bedside". To Dr. Xiaoping Xiong, I wish to thank for his help on our early manuscripts and always keeping me honest. I also wish to thank Dr. Rick Boop and Dr. Robert Sanford for their interest in our work in those early years and their subsequent collaboration. I do not believe we would have been nearly as successful without their support. I also wish to thank Matt Scoggins and Scott Malone, whose advice and friendship always made my life a little bit easier. Finally, I wish to thank Ping Zou Stinnett for her patience and help. Without her I do not believe I would have survived those early days. 


\begin{abstract}
In this dissertation, we outline our efforts to introduce an advanced MRI imaging technique called Diffusion Tensor Imaging (DTI) to the pediatric brain tumor population. We discuss the theory and application of DTI as it was performed in a series of translational investigations at St. Jude Children's Research Hospital. We present evidence of how the introduction of this technique impacted diagnosis, and treatment. And finally, we demonstrate how DTI was used to investigate cognitive morbidities associated with cancer treatment and how this research provided insight into the underlying pathophysiology involved in the development of these treatment sequela.

This research has generated important insights into the fundamental causes of neuroanatomical and cognitive deficits associated with cancer and cancer therapy. The use of DTI has permitted us to identify potential targets for improved radiological and surgical techniques as well as targets for pharmacological and behavioral interventions that might improve cognitive function in cancer survivors. The discoveries here afford us an opportunity to reduce the negative effects of cancer therapy on patients treated in the future while maintaining successful survival rates.
\end{abstract}




\section{Table of Contents}

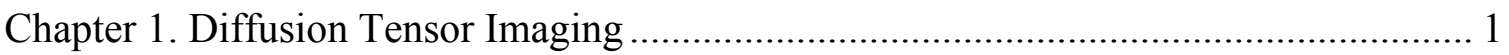

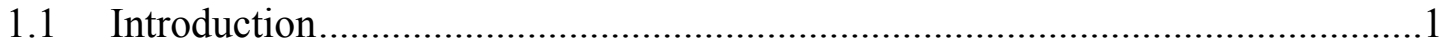

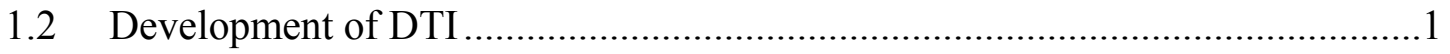

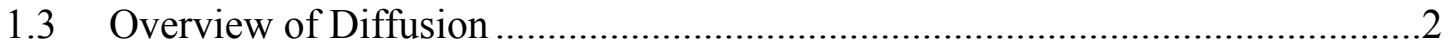

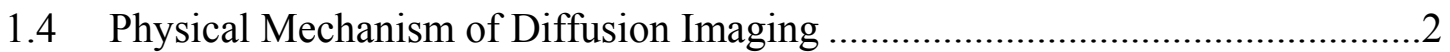

1.5 Assessment of White Matter Pathways Using DTI ..........................................

1.6 Application of Diffusion Tensor Imaging in Biological Tissues........................10

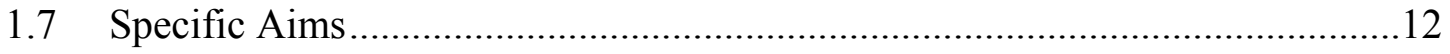

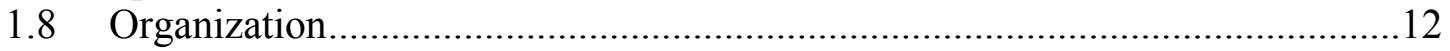

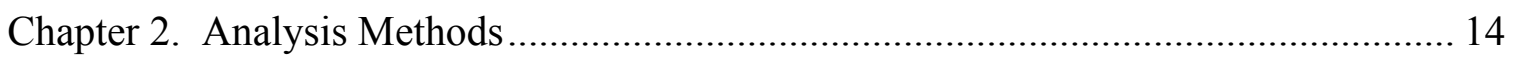

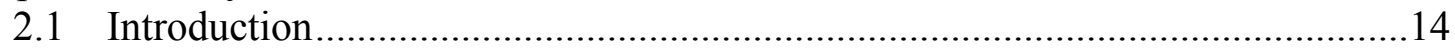

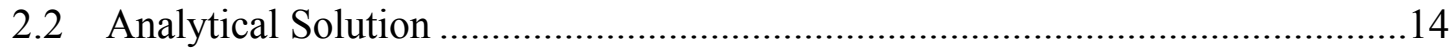

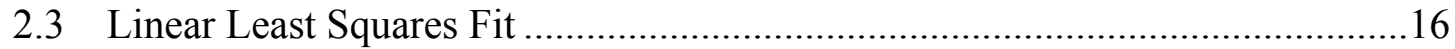

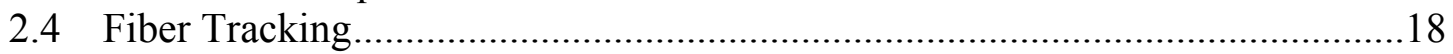

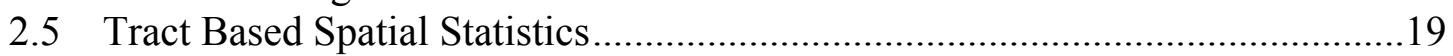

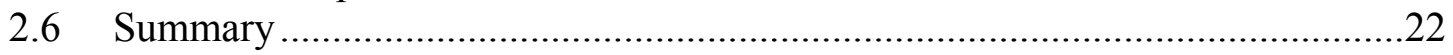

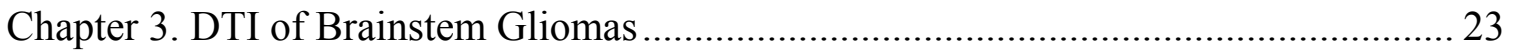

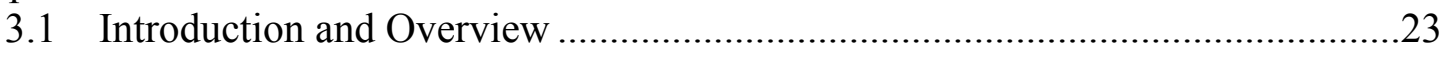

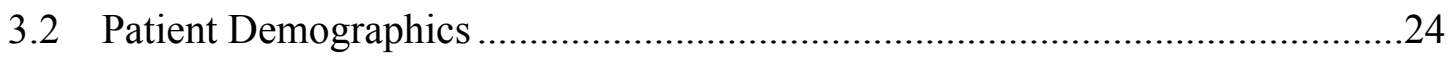

3.3 Conventional Magnetic Resonance Imaging …………..................................24

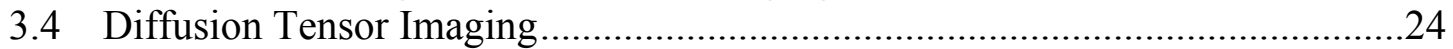

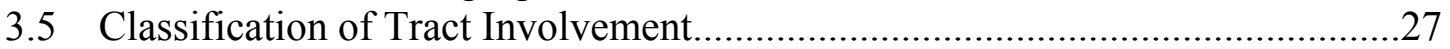

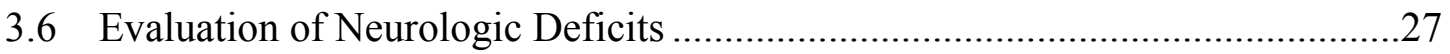

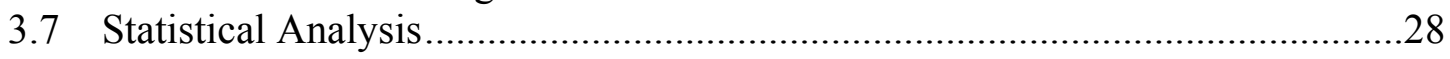

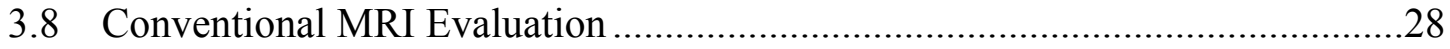

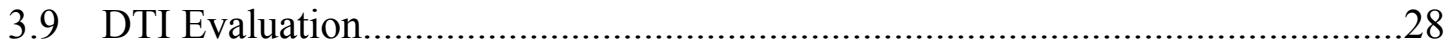

3.10 White Matter Tract Involvement ………………............................................

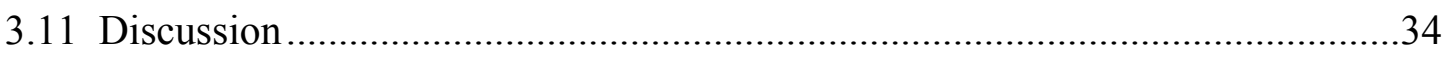

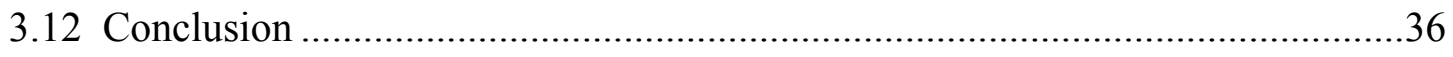

Chapter 4. Axonal Degeneration in Pediatric Brain Tumor Patients................................. 37

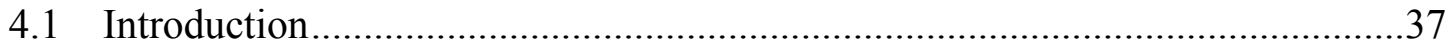

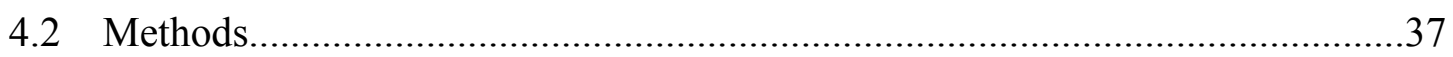

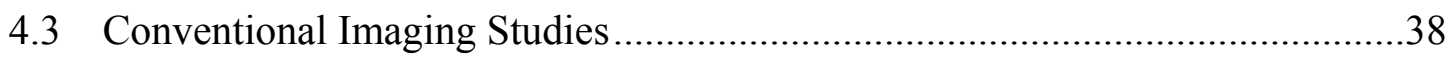

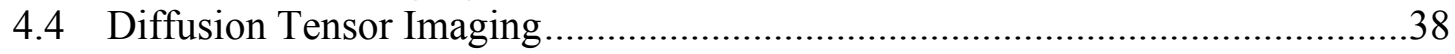

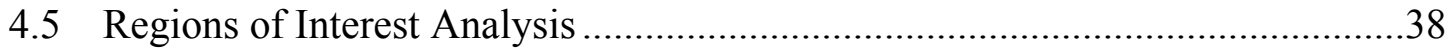

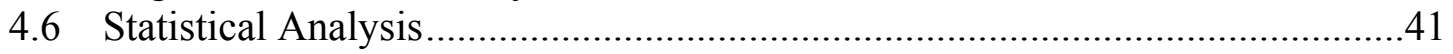

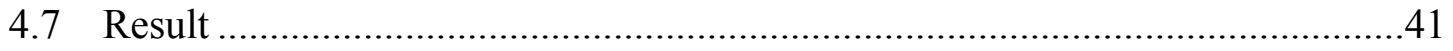

4.7.1 Conventional MR Imaging ................................................................

4.7.2 Color Map Analysis ...........................................................................44

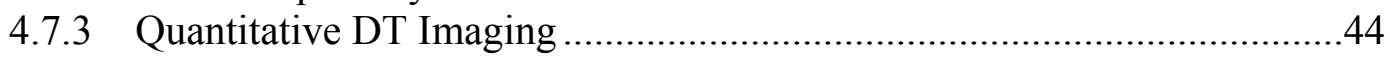




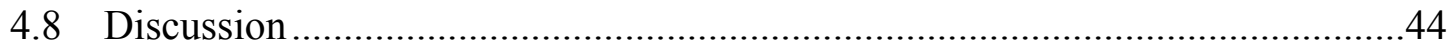

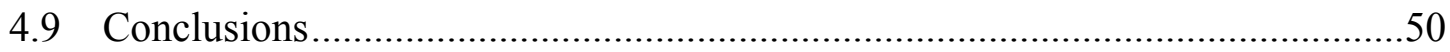

Chapter 5. Utility of DTI in Surgical Planning ........................................................... 51

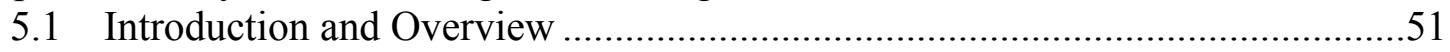

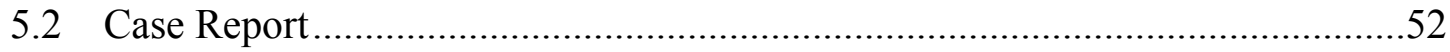

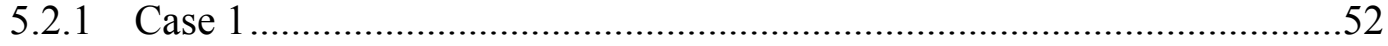

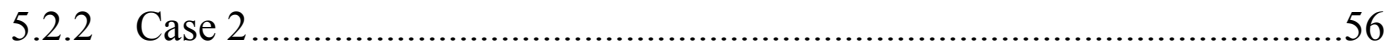

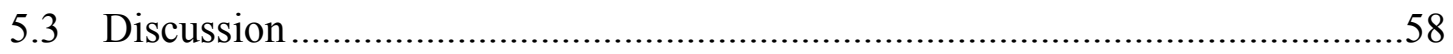

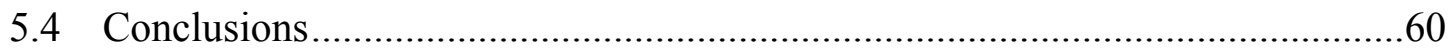

Chapter 6. Evaluation of Medulloblastoma Therapy with DTI ....................................... 61

6.1 Medulloblastoma: Overview and Contemporary Therapy ...................................61

6.2 Neurocognitive Sequelae of Medulloblastoma Therapy.......................................61

6.3 The Ventral Visual System and Orthographic Processing in the Human

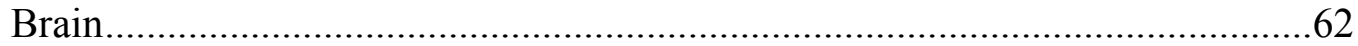

6.4 Radiation Dose Distribution in Medulloblastoma Patients...................................64

6.5 DTI in Evaluation of Therapy-Induced Changes...............................................64

6.6 Experimental Design and Methods .................................................................66

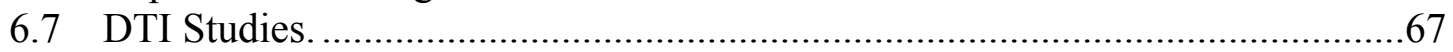

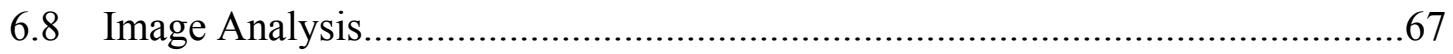

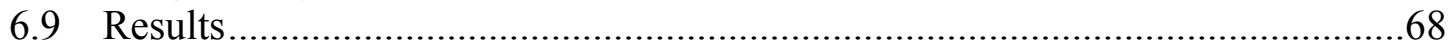

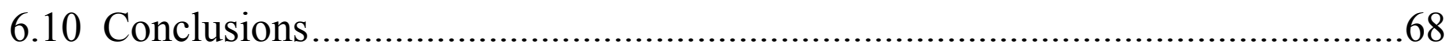

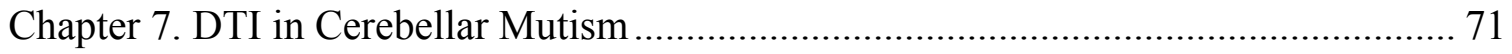

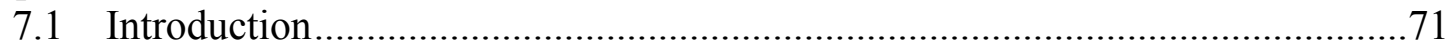

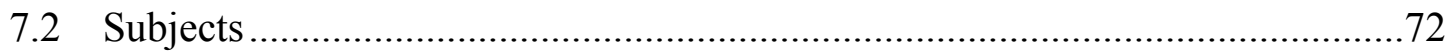

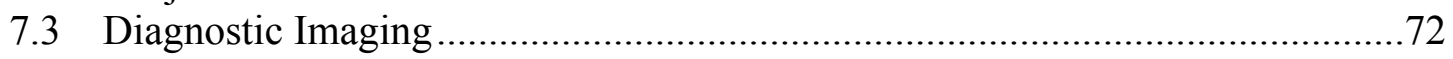

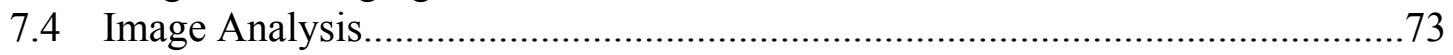

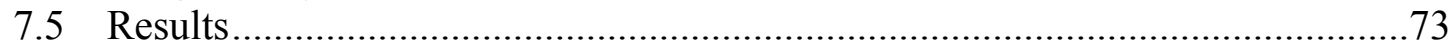

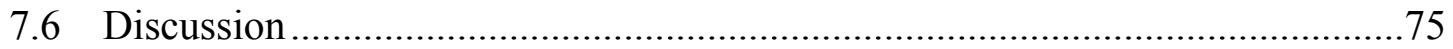

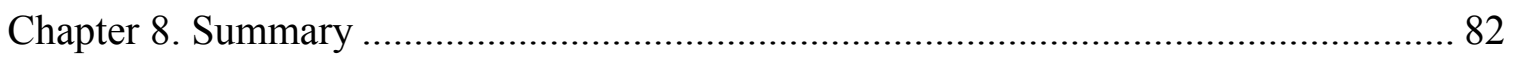

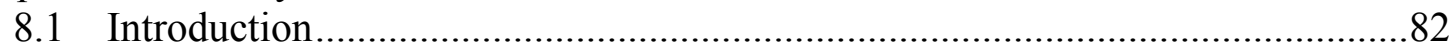

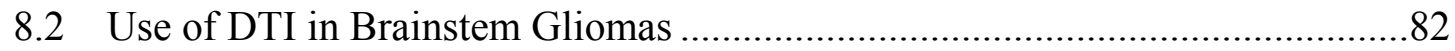

8.3 Axonal Degeneration in Pediatric Brain Tumor Patients ....................................83

8.4 Utility of DTI in Surgical Planning .............................................................8

8.5 Evaluation of Medulloblastoma Therapy with DTI.............................................84

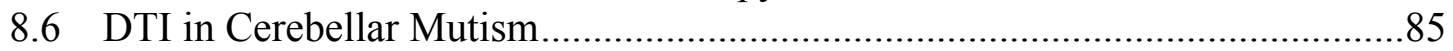

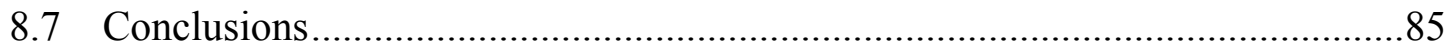

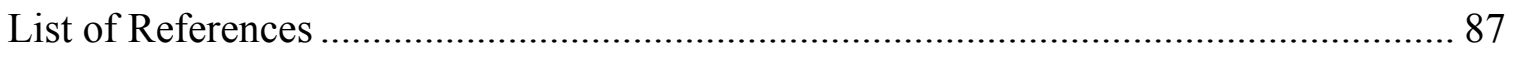

Appendix A. Modified SPM99 Poldrack Diffusion Calculation ..................................... 102

Appendix B. SPM2 DTI Preprocessing Toolbox ………............................................... 110 
Appendix C. Modified SPM2 (Volkmar) DICOM Convert Files

Appendix D. SPM2 Volkmar Diffusion Regression................................................... 132

Appendix E. TBSS Preprocessing Batch Files for Parallel Processing ........................... 141

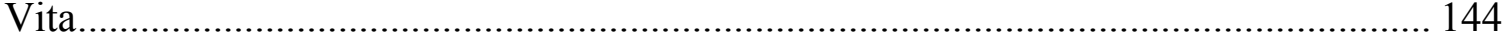




\section{List of Tables}

Table 3.1 Comparison of conventional MRI and DTI measures in 7 pediatric patients with brainstem gliomas.

Table 3.2 Color map assessment of diffusion tensor images of brainstem white matter in pediatric patients with brainstem gliomas.

Table 3.3 Neurological deficits in 7 pediatric patients with brainstem gliomas..............33

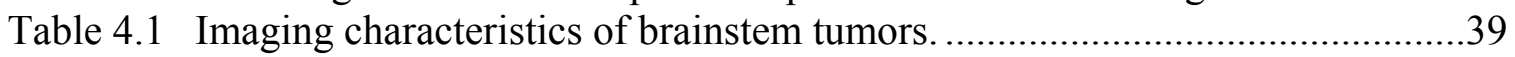

Table 4.2 Analysis of colormap at the level of the brainstem......................................46

Table 4.3 Comparison of median FA and ADC values obtained in the sensory tracts of volunteers and patients.

Table 4.4 Comparison of median FA and ADC values obtained in the motor tracts of volunteers and patients.

Table 6.1 Langston modification of Chang staging for medulloblastoma.....................62

Table 6.2 SJMB03 treatment schemes based on risk assessment. ..............................63

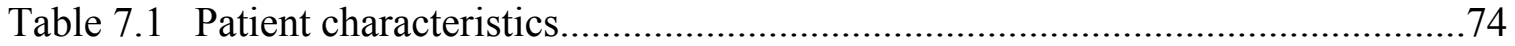

Table 7.2 Frequency of T2 involvement in baseline imaging.................................... 76

Table 7.3 Frequency of T2 involvement in immediate post-operative imaging..............77 


\section{List of Figures}

Figure 1.1 Phase dispersion of transverse magnetization..............................................

Figure 1.2 Stejskal-Tanner spin echo pulse sequence..............................................

Figure 1.3 Diffusion tensor color map of the averaged brains of 6 medulloblastoma

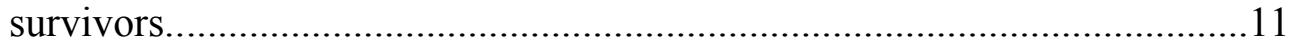

Figure 2.1 Outline of the general procedure for performing Tract-Based Spatial Statistics (TBSS) .............................................................................20

Figure 3.1 MRI and DTI images of a 15-year-old patient with diffuse brainstem

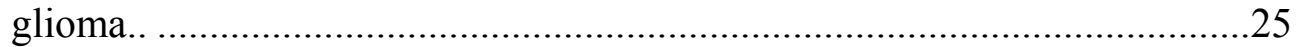

Figure 3.2 Axial diffusion tensor color maps of the brainstem at the level of the middle cerebellar peduncles...............................................................26

Figure 3.3 Axial diffusion tensor color maps demonstrating tract invasion.................31

Figure 3.4 Diffusion tensor imaging parameters for major white matter tracts in the

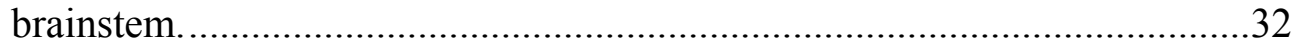

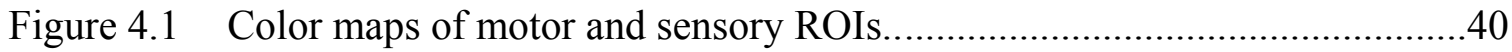

Figure 4.2 White matter images of representative focal tumor.................................42

Figure 4.3 Diffusely infiltrating medullary tumor................................................ 43

Figure 4.4 Classic pontine glioma.....................................................................45

Figure 5.1 Case 1 showing unenhanced and contrast-enhanced T1-weighted sagittal (upper) and axial (lower) images obtained at the referring hospital.............53

Figure 5.2 Case 1 showing diffusion tensor imaging colormaps. ................................55

Figure 5.3 Case 2 showing preoperative T2-weighted (left) and raw (center) and masked (right) DT axial images obtained in adjacent slices at the pontomedullary junction.

Figure 5.4 Conventional and DT images obtained in a 9-year-old female patient with a diffusely infiltrative pontine glioma.

Figure 6.1 Combined MRI and radiation dosimetry map shows that the splenium of the corpus callosum is disproportionately affected by the radiation therapy.....

Figure 6.2 Example of time course of FA changes of a single ROI of the splenium in three month intervals over one year for 8 random subjects.

Figure 7.1 Depicts splay between SCP's (A) and above/below ratio (B) in a patient

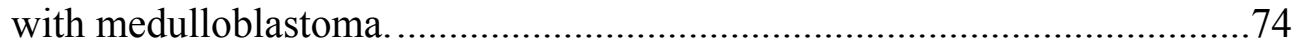

Figure 7.2 Post-operative scan of patients with CMS ............................................ 78 


\section{List of Abbreviations}

$\mathrm{AC}$

ADC

ALL

$\mathrm{CC}$

CNS

CSF

CT

DTI

EPI

FA

fMRI

FMRIB

FOV

FSL

IRB

MNI

MRI

PC

PT

PDD

RA

ROI anterior commissure

apparent diffusion coefficient

acute lymphoblastic leukemia

corpus collosum

central nervous system

cerebral spinal fluid

computed tomography

diffusion tensor imaging

echo planar imaging

fractional anisotropy

functional magnetic resonance imaging

functional MRI of the brain

field of view

FMRIB's software library

institutional review board

Montreal Neurological Institute

magnetic resonance imaging

posterior commissure

patient

principal diffusion direction

relative anisotropy

region of interest 
SJMB03

SNR

SPM

TBSS

TE

TR

XRT
St. Jude Medulloblastoma Protocol '03

signal to noise ratio

statistical parametric mapping

tract-based spatial statistics

echo time

repetition time

radiation therapy 


\section{Chapter 1. Diffusion Tensor Imaging}

\subsection{Introduction}

Historically, a diagnosis of a pediatric brain tumor was uniformly fatal. Only within the last 40 years have innovations in technology, pharmacology and surgical techniques afforded children a reasonable chance for survival. One of the most important innovations was the introduction of advanced imaging modalities, such as Computed Tomography (CT) and Magnetic Resonance Imaging (MRI). These modalities allowed physicians and surgeons to peer into the "black box" of the skull and detect structural abnormalities and tumors for the first time. Prior to these techniques, clinicians had no direct way to visualize the brain. With improved imaging, clinicians soon began to develop effective strategies for surgical resection and medical treatments. Imaging studies also began to reveal subclasses and subtypes of tumors and that allowed researchers to drive their investigation in new and novel ways. This led to a dramatic shift in survival curves among children with infratentorial tumors. It was with this history in mind, that I turned my attention to investigate and introduce advanced imaging tools that would improve the diagnosis and treatment of pediatric brain tumor patients. Concurrently, I developed a series of studies with these new set of tools to evaluate morbidities associated with cancer treatment.

In this dissertation, I outline our efforts to introduce one such advanced MRI imaging technique called Diffusion Tensor Imaging (DTI) to the pediatric brain tumor population. I will discuss the theory and application of DTI as it was performed in a series of translational investigations at St. Jude Children's Research Hospital. I will present evidence of how the introduction of this technique impacted diagnosis, and treatment. And finally, I will demonstrate how DTI was used to investigate cognitive morbidities associated with cancer treatment and how this research provided insight into the underlying pathophysiology involved in the development of these treatment sequela.

\subsection{Development of DTI}

In the early 1970's, Lauterbaur and Damadian produced the first Magnetic Resonance Imaging (MRI) studies and demonstrated the difference in the relaxation times $\mathrm{T} 1$ and $\mathrm{T} 2$ between normal and cancerous tissues. Almost immediately, the medical importance of MRI became apparent and medical research centers began the enormous task of developing MRI systems for hospital use. Over the following 30 years, there was an explosion of technological and scientific advancement in the field of MRI. Scanners became faster and more sensitive, and MR phenomena that initially created problems and artifacts, soon became the basis for new imaging techniques [1]. Improvements in MR imaging hardware and computer capabilities led to faster image acquisition techniques and allowed imaging of rapidly changing physiological processes [2]. For example, with the advent of Echo Planar Imaging (EPI), newer clinical 
applications such as diffusion, perfusion and Blood Oxygen Level Dependant (BOLD) imaging became available.

These advances in MR gradient design and pulse sequences opened up the possibility of studying white matter pathways in vivo. Diffusion Tensor Imaging (DTI) is a non-invasive imaging technique that measures water diffusion in tissue to infer orientation of white-matter axonal fibers in vivo. Until the advent of DTI, the only reliable techniques used to study axonal pathways were invasive procedures that are only feasible in primate or post-mortem human brains [3-5]. The utility of DTI lies in the many types of images that can be calculated from the information contained in the tensor formalism. These maps can provide scalar information regarding the magnitude and spatial aniosotropy of diffusion in a tissue, as well as vector maps that describe directionality of diffusion. In this chapter, we will describe the physics of DTI and relate that to the physical properties of tissue.

\subsection{Overview of Diffusion}

"Molecular diffusion is a stochastic thermal phenomenon characterized by Brownian motion" [6]. This process can be restricted or unrestricted. In biological tissue, diffusion of water molecules is affected by microstructures and macromolecules. These structures often restrict diffusion asymmetrically. If diffusion is greater in one direction, then the diffusion is said to be anisotropic. Diffusion that is equal in all directions is isotropic.

Interestingly, in MRI, diffusion of water molecules in the presence of a heterogeneous gradient magnetic field causes changes in the phase dispersion of the transverse magnetization (Fig 1.1). This phase dispersion translates into attenuated MRI signal. The degree of signal loss is related to the physical and physiological environment of the biological tissue [6]. Several MRI techniques have been designed to exploit these tissue diffusion properties. Diffusion Tensor Imaging (DTI) is one of these techniques that measures the "spatial orientation dependency" of the diffusion process [6].

\subsection{Physical Mechanism of Diffusion Imaging}

To better understand the effects of diffusion on signal attenuation, we need to discuss the underlying MRI data acquisition methods used to exploit tissue diffusion property differences. Spin-spin relaxation, (or T2), is the process in which transverse magnetization decays by loss of phase coherence. For example, in homogenous tissue, the spin sees a magnetic field which is increased or decreased due to the magnetic moment of the other spins [6]. Differences in angular precession can be the result of spatial or temporal variations in the local magnetic field. Additional variations can be caused by the nuclear moment of neighboring atoms or due to inhomogeneties in the externally applied field. The result is the same in all cases, dephasing of the sample spins. 


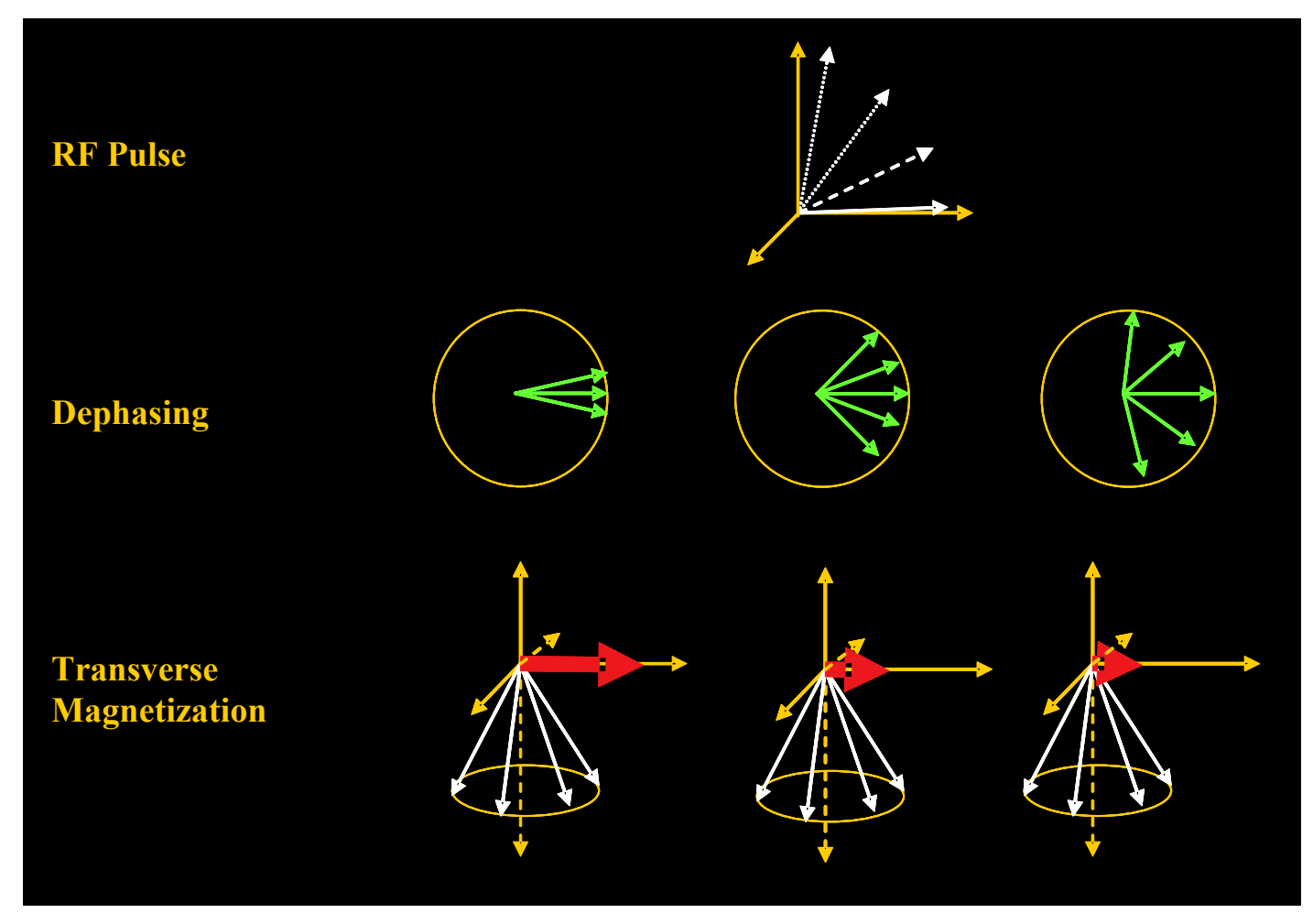

Figure 1.1 Phase dispersion of transverse magnetization. This figure describes the effect of dephasing of spins on the transverse magnetization during a diffusion imaging sequence. After the RF pulse is applied net magnetization (red arrow) is tipped into the transvers plane, as time progresses, the phase of the spins become inhomogeneous. This leads to a decrease in the overall strength of the net transverse magnetization (red arrow) and thus MRI signal loss. 
The physical implications of this type of interaction can be explained first by describing spin-spin relaxation at the local level. An isolated spin is only affected by the externally applied magnetic field. As spins move together, (due to random thermal motion), their magnetic fields begin to interact. Some spins will increase the field and some will decrease the field. As soon as the spins move apart, their fields no longer interact and begin to precess at the Lamour (external) frequency again. However, because of the brief increase or decrease in precession during their interaction, the spins will be slightly out of phase. It is these random interactions between spins that cause a cumulative loss in phase that result in an overall loss of signal.

In a pulse NMR experiment, transverse magnetization will decay with an exponential time constant T2. The rate of signal loss is given as Eq 1.1. This decay rate is usually much greater than or equal to the longitudinal recovery rate (T1). The value of $\mathrm{T} 2$ is described as the time after excitation when the signal amplitude has lost $63 \%$ of its strength. And unlike T1, T2 is unrelated to field strength.

$$
\begin{aligned}
& \frac{d M_{x y}}{d t}=-\frac{M_{x y}}{T 2}+\gamma(M \times B)_{x y} \text { (in rotating frame, external field cancels) } \\
& \frac{d M_{x y}}{d t}=-\frac{M_{x y}}{T 2} \\
& M_{x y}=M_{0} e^{\frac{-t}{T 2}}
\end{aligned}
$$

Pure $\mathrm{T} 2$ decay $(\mathrm{T} 2 *)$ is the sum total of both random interactions and fixed effects (Eq 1.2). For example, in the static condition (such as bone) where fixed effects truly dominate, T2 is too short for MR measurement with commonly used techniques for in vivo imaging. While in pure water, random effects dominate and $\mathrm{T} 2$ decay takes several seconds. Normally one of these mechanisms will dominate. In clinical MRI, the value of T2 is primarily determined by intra-voxel dephasing due to the chemical composition of the sampled tissue, since molecular diffusion is restricted in healthy tissue. Every tissue has a different magnetic susceptibility which distorts the externally applied magnetic field at tissue borders. Tissue rich in paramagnetic molecules, (venous blood for instance), will produce large fields in the applied field relative to diamagnetic tissue (such as brain tissue). Thus the protons of water molecules in tissue come under the influence of the extra magnetic fields produced so that dephasing occurs in tens of ms.

$$
\frac{1}{T 2^{*}}=\frac{1}{T 2_{\text {random }}}+\frac{1}{T 2_{\text {fixed }}}
$$

The transverse magnetization decay rate $(1 / \mathrm{T} 2 *)$ is also influenced by the recovery rate constant along the $\mathrm{z}$ axis $(1 / \mathrm{T} 1)$, as well as the external field inhomogeneties $\left(\gamma \Delta \mathrm{H}_{0}\right)(\mathrm{Eq} 1.3)$. To offset the contribution of $1 / \mathrm{T} 1$ to $\mathrm{T} 2 *$ decay, a long interval between 'rf' pulse excitations (TR) is used in most T2 techniques. As TR $\rightarrow \infty$, 
$\mathrm{T} 1$ is allowed to recover completely, thus minimizing any contribution of $\mathrm{T} 1$ to $\mathrm{T} 2 *$. On the other hand, the external field inhomogeneties, $\mathrm{T} 2$ fixed, is both machine and sample dependant [7]. These factors often lead to faster T2* decay than expected. However, unlike $\mathrm{T} 2_{\text {random }}, \mathrm{T} 2_{\text {fixed }}$ is recoverable. That is to say, the effects of $\mathrm{T} 2_{\text {fixed }}$ decay can be reversed by the application of the spin-echo technique. This well known technique uses a second "refocusing" rf pulse to recover $\mathrm{T} 2_{\text {fixed }}$ signal loss. Though this technique offers of method for $\mathrm{T}_{\text {fixed }}$ recovery, it is not absolute. For example, if the spins move, due to random motion, in the interval between the application of the first and second rf pulse, they will experience imperfect refocusing. Thus recovery can only be complete if the gradient does not change over time.

$$
\frac{1}{T 2^{*}} \cong \frac{1}{(2 T 1)}+\frac{1}{T 2_{\text {random }}}+\gamma \Delta H_{0}
$$

In all cases, however, $\mathrm{T} 2_{\text {random }}$ decay is not affected by the spin-echo technique. Random fluctuations in the local intrinsic fields do not stay fixed and are therefore irreversible. Thus intrinsic loss predominates in the spin-echo technique. Therefore we measures $\mathrm{T} 2$ rather than $\mathrm{T} 2 *$ at $\mathrm{TE}(\mathrm{Eq} 1.4)$.

$$
M_{x y}(t)=M_{x y}(0)\left\{\begin{array}{l}
e^{-t / T 2^{*}} \\
e^{-t / T 2} e^{-(T E-t) / T 2_{\text {fixed }}} \\
e^{-t / T 2} e^{-(t-T E) T 2_{\text {fixed }}}=e^{-t / T 2^{*}} e^{T E / T 2_{\text {fixed }}}
\end{array}\right\} \begin{gathered}
0<t<\tau \\
\tau<t<2 \tau=T E(\mathrm{Eq} 1.4) \\
t>2 \tau=T E
\end{gathered}
$$

Observations have demonstrated a diffusion exponent dominates the signal decay for longer times [7]. To account for this phenomenon we modify Eq 1.1 by adding terms to describe the contribution of transverse magnetization due to anisotropic diffusion of free water [1].

$$
\frac{d M_{x y}}{d t}=-\frac{M_{x y}}{T 2}-i \gamma \overrightarrow{G(t)} \vec{r} M_{x y}+\stackrel{\vec{D}}{\nabla} \nabla M_{x y}
$$

Where $\mathrm{D}$ is an apparent diffusion coefficient, $\nabla$ is a column vector defined as: $\nabla^{T}=\left(\vec{i} \frac{d}{d x}, \vec{j} \frac{d}{d y}, \vec{k} \frac{d}{d z}\right)^{T}, \gamma$ is the gyromagnetic constant of the hydrogen proton, and $\mathrm{G}$ is the strength of the applied gradient. The solution for this modified Bloch equation can be found by first solving the Eq 1.1 without the diffusion term [1].

$$
M_{x y}(t)=M_{x y}(0) \exp \left(-i \gamma \vec{r} \int_{0}^{t} \vec{G}\left(t^{\prime}\right) d t^{\prime}-\frac{t}{T 2}\right)
$$

Substituting: $\vec{k}(t)=\gamma \int_{0}^{t} \vec{G}\left(t^{\prime}\right) d t^{\prime}$ 


$$
M_{x y}(t)=M_{x y}(0) \exp \left(-i \vec{k}(t) \vec{r}-\frac{t}{T 2}\right)
$$

Substituting: $M_{x y}(0)=A(t)$

$$
M_{x y}(t)=A(t) \exp \left(-i \vec{k}(t) \vec{r}-\frac{t}{T 2}\right)
$$

Introducing this in to Eq 1.5 we see:

$$
\frac{d A(t)}{d t}=\nabla^{T}(\vec{D} \nabla A(t))=-k^{\vec{T}}(t) \vec{D} k(\vec{t}) A(t)
$$

Substituting: $\nabla=-i \vec{k}(t)$

$$
M_{x y}(t)=M_{x y}(0) \exp \left[-\int_{0}^{t}\left(\vec{k}^{T}\left(t^{\prime}\right) \vec{D} \vec{k} t^{\prime}\right) d t^{\prime}\right] \exp \left(-i \vec{k}(t) \vec{r}-\frac{1}{T 2}\right)
$$

This continuity equation describes the effects of the apparent anisotropic diffusion (first exponent) and the rate of change due to precession and relaxation (second exponent). In practice, these diffusion effects are suppressed by a series of $\pi$-pulses prior to the readout gradient. However, as we have already discussed, recovery is complete only if the phase gradient does not change. Taking Eq 1.8, we can derive the effects of a gradient on the measured signal using a bipolar pulse of duration $2 \tau_{\mathrm{b}}$ [7].

$$
\int_{0}^{t} G\left(t^{\prime}\right) d t^{\prime}=\left\{\begin{array}{lc}
G t^{\prime \prime} & \text { for }: t^{\prime \prime}<\tau_{b} \\
-G\left(t^{\prime \prime}-2 \tau_{b}\right) & \text { for }: \tau_{b}<t^{\prime \prime}<2 \tau_{b}
\end{array}\right\}
$$

Solving Eq 1.8, for $\mathrm{t}=2 \tau_{\mathrm{b}}$, we find:

$$
\ln A_{b i}\left(2 \tau_{b}\right)=-D \gamma^{2} \int_{0}^{\tau_{b}} G^{2} t^{\prime \prime 2} d t^{\prime \prime}-D \gamma^{2} \int_{0}^{2 \tau_{b}} G^{2}\left(t^{\prime \prime}-2 \tau_{b}\right)^{2} d t^{\prime \prime}
$$

Substituting: $\mathrm{u}=2 \tau_{\mathrm{b}}-\mathrm{t}$ " for the second integral gives the solution:

$$
\ln A_{b i}\left(2 \tau_{b}\right)=-\frac{2}{3} D \gamma^{2} G^{2} \tau_{b}^{3}
$$

This demonstrates that signal loss due to diffusion is generally governed by the square of the gradient $(\mathrm{G})$ and the cube of the gradient duration [7]. Thus applying the bipolar pulse along any axis gives a measured signal proportional to: 


$$
M(T E) \propto \exp \left(-\frac{2}{3} D \gamma^{2} G^{2} \tau_{b}^{3}-T E / T 2\right)
$$

When Eq 1.12 is solved for the spin echo case (a single $\pi$-pulse applied at time $\tau$ to reset phase clock) (Fig 1.2), we derive, [7].

$$
b=\ln A_{b i}=-\frac{2}{3} D \gamma^{2} G^{2} \delta^{2}\left(\Delta-\frac{1}{3} \delta\right)
$$

This ' $b$ ' factor describes the net effect of diffusion sensitization using bipolar gradients and must be calculated appropriately for the method. This technique can be used to determine the diffusion weighting along the direction of an applied gradient. In practice this is accomplished by using a spin echo technique similar to the StejskalTanner sequence. As seen in Fig 1.2, the two lobe gradients $(\delta)$ are inserted between the excitation and refocusing pulse and the acquisition gradient, respectively [1]. This experiment is repeated using a fixed echo time, with varying diffusion gradient amplitude in the same direction for two or more scans. These scans are then solved, as system of linear equations, for the diffusion coefficient (D) for a fixed direction.

So as we have seen, spins undergoing random thermal motion in a gradient field undergo a phase shift which generates imperfect refocusing and thus signal loss [1]. In tissues, diffusion is restricted by the presence of cellular and subcellular structures [8] and leads to direction dependant signal changes. The methods described in this section demonstrate a practical method for measuring these diffusion differences.

\subsection{Assessment of White Matter Pathways Using DTI}

As we have discussed, random thermal motion of spins in a gradient field cause a phase shift of their transverse magnetization [8,9]. In a spin-echo sequence this means that the rephasing will be incomplete after the refocusing pulse because the individual spins will have changed position. This leads to a signal (S) attenuation which is proportional to the gradient history (i.e., direction, duration, and strength) and the diffusion coefficient of the protons in tissue (D). A simplified mathematical description of the signal loss due to the diffusion process is given by Eq 1.15. To determine the diffusion coefficient, it is necessary to obtain measurements of at least two different $b$ values. The $b$ value is a function of the gyromagnetic constant of the hydrogen proton $(\gamma)$, the strength of the applied gradient $(\mathrm{G})$ and its duration $(\delta)$. The value of $b$ is changed by changing the strength of the gradient $(\mathrm{G})$. One measurement is carried out with no applied field with an associated signal loss of $\mathrm{S}_{0}$.

$$
\ln \left[S_{i}\right]=\ln S_{0}-\vec{D}\left[\vec{b}_{i}\right]^{T}
$$



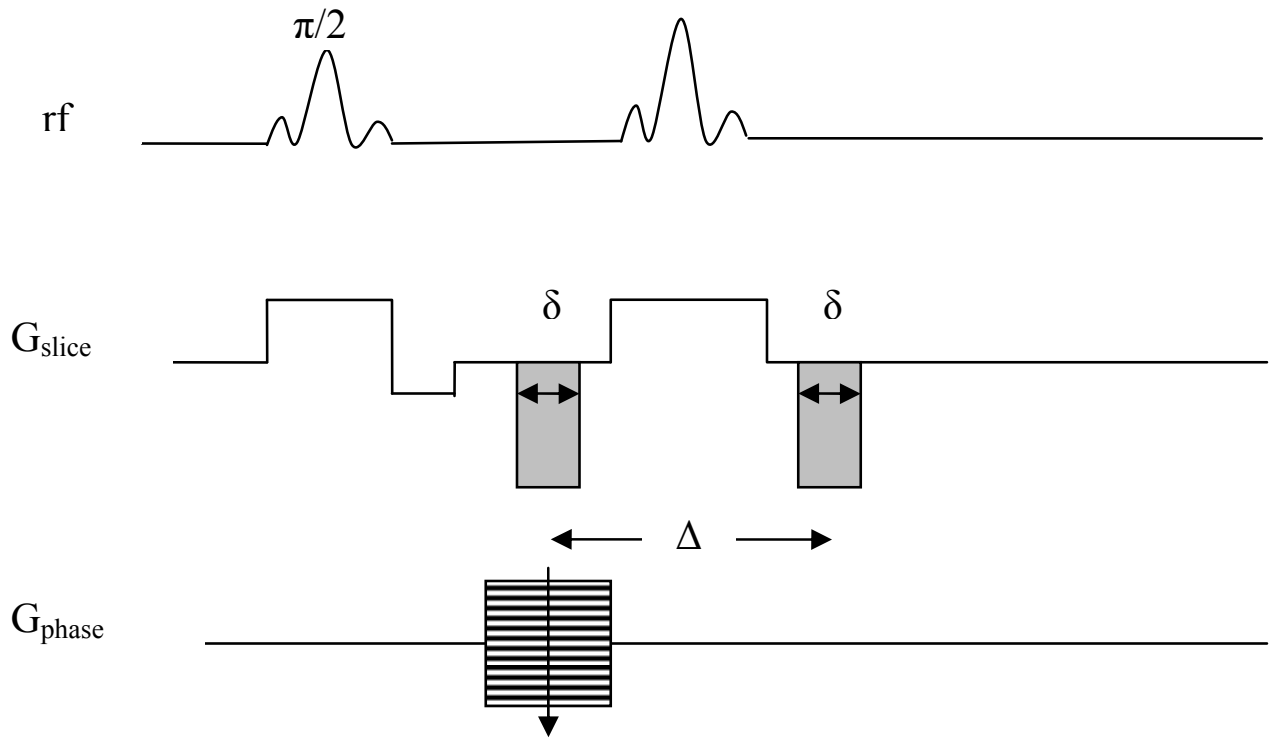

$\mathrm{G}_{\text {readout }}$

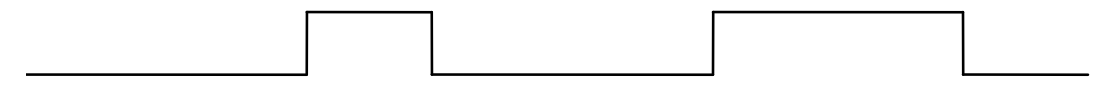

Figure 1.2 Stejskal-Tanner spin echo pulse sequence. 
In the brain, water molecules diffuse preferentially parallel to nerve fibers rather than across them. Thus if the diffusion orientation were known, three measurements along the three perpendicular directions would be sufficient to provide the orientation of the diffusion tensor. However, in biological samples, diffusion orientation is generally not known and it is necessary to know more than 3 gradient directions. Three rules must be must be followed to guarantee a valid tensor calculation when selecting the optimal gradient vector scheme [10]. 1) No two gradients vectors can be the parallel or antiparallel of another vector. 2) If three vectors are coplanar, then the remaining vectors must be linearly independent. 3) Finally, no four vectors may be coplanar. In general this means no less than 6 directions are necessary to calculate a valid tensor [11]. Thus by measuring the diffusion of water molecules in six or more directions we can detect the orientation of the underlying nerve fiber tracts of the brain. In Diffusion Tensor Imaging (DTI), a set of diffusion-weighted images are acquired and analyzed to produce a tensor (T) which describes the diffusion associated with each individual volume element (voxel) (Eq 1.16). Two measures that are commonly used can be derived from the tensor, Apparent Diffusion Coefficient (ADC) and Fractional Anisotropy (FA).

$$
T=\left(\begin{array}{ccc}
D_{x x} & D_{x y} & D_{x z} \\
D_{y x} & D_{y y} & D_{y z} \\
D_{z x} & D_{z y} & D_{z z}
\end{array}\right)
$$

The ADC $\left(\mathrm{mm}^{2} / \mathrm{s}\right)$ is a measure of the magnitude of the diffusion. The calculated diffusion is called "apparent" because in the ratio method it is assumed that the diffusion process is not restricted by the tissue septa and is isotropic [1]. The average apparent diffusion is measured using diffusion-sensitizing gradients applied along 6 non-colinear and non-coplanar directions. The directions are varied in such a way that the optimal off diagonal terms cancel and the diagonal terms are retained with the same weighting $(\mathrm{Eq}$ 1.17) [1].

$$
\int_{0}^{t} k_{i}\left(t^{\prime}\right) D_{i j} k_{j}\left(t^{\prime}\right) d t=\Delta_{i j}=0 ; \quad \text { with } i \neq j
$$

The trace of the diffusion matrix thus gives a full representation of the apparent diffusion in the voxel (Eq 1.18).

$$
A D C=D_{x x}+D_{y y}+D_{z z}
$$

The FA is a dimensionless quantity that is a measure of the anisotropy of water diffusion (Eq 1.19). It is calculated by solving the diffusion tensor for its eigenvectors and corresponding eigenvalues. Since the second order tensor is real and symmetric, the eigenvalues of $\mathrm{T}_{\mathrm{mn}}$ will be real, and the eigenvectors corresponding to the distinct eigenvalues will be orthogonal [12]. If the diffusion is equal in all directions the FA is approximately 0 . Conversely, if the diffusion is principally along only one direction, the FA approaches 1 . 


$$
\begin{aligned}
& \bar{T}_{m n}=\left(\begin{array}{ccc}
\lambda_{1} & 0 & 0 \\
0 & \lambda_{2} & 0 \\
0 & 0 & \lambda_{3}
\end{array}\right) \\
& F A=\sqrt{\frac{3}{2}} \frac{\sqrt{\left(\lambda_{1}-<\lambda>\right)^{2}}+\sqrt{\left(\lambda_{2}-<\lambda>\right)^{2}}+\sqrt{\left(\lambda_{3}-<\lambda>\right)^{2}}}{\sqrt{\lambda_{1}^{2}+\lambda_{2}^{2}+\lambda_{2}^{2}}}
\end{aligned}
$$

where $\langle\lambda\rangle=\frac{\lambda_{1}+\lambda_{2}+\lambda_{3}}{3}$

The benefit of DTI over conventional scalar imaging is the ability to depict orientation information in the image. For example, a color map can be created, based on the eigenvectors and eigenvalues, to convey orientation information about prominent white matter tracts (Fig 1.3). A color is assigned to each voxel, which corresponds to the principal direction of diffusion in that voxel. The limitation of this image is that connectivity of the pixels cannot be demonstrated, especially between slices. To appreciate the true 3-D trajectories of the white matter pathway, computer aided tracking algorithms have been developed.

\subsection{Application of Diffusion Tensor Imaging in Biological Tissues}

Several important findings related to the FA in white matter have particular relevance to the application of DTI to the investigation of pediatric brain tumor patients. A study of children (ages 7 months-12 years) showed that the FA of white matter in the major motor and sensory and transcollosal pathways are essentially constant beyond age 4 , [13], and recent data published in abstract form showed that the FA of the corpus callosum remains stable through age 60 [14]. Hermove et al. (2006) demonstrated that FA changes followed a three step process of rapid change in size and shape during the first 12 months, slow modification in the second 12 months and relative stability after 24 months. Thus, for healthy subjects in the age range we studied in this dissertation, we expected the FA to be constant. Also, though the physiological and functional significance of differences in water diffusion parameters is not known $[15,16]$, a growing body of evidence suggests that the FA reflects functionally significant properties of white matter.

In evaluation of DTI to detect axonal injury, two studies have demonstrated that DTI reliably detects experimental injury and can be correlated to both histological and neurological disability in animal models. MacDonald, et al. (2007) first demonstrated that in regions of verified axonal injury in mouse models, DTI was in fact sensitive with 24 hours of the injury [17]. A follow up study by Macdonald, et al, demonstrated, that changes in Relative Anisotropy (RA) corresponded to the evolution of isolated axonal 


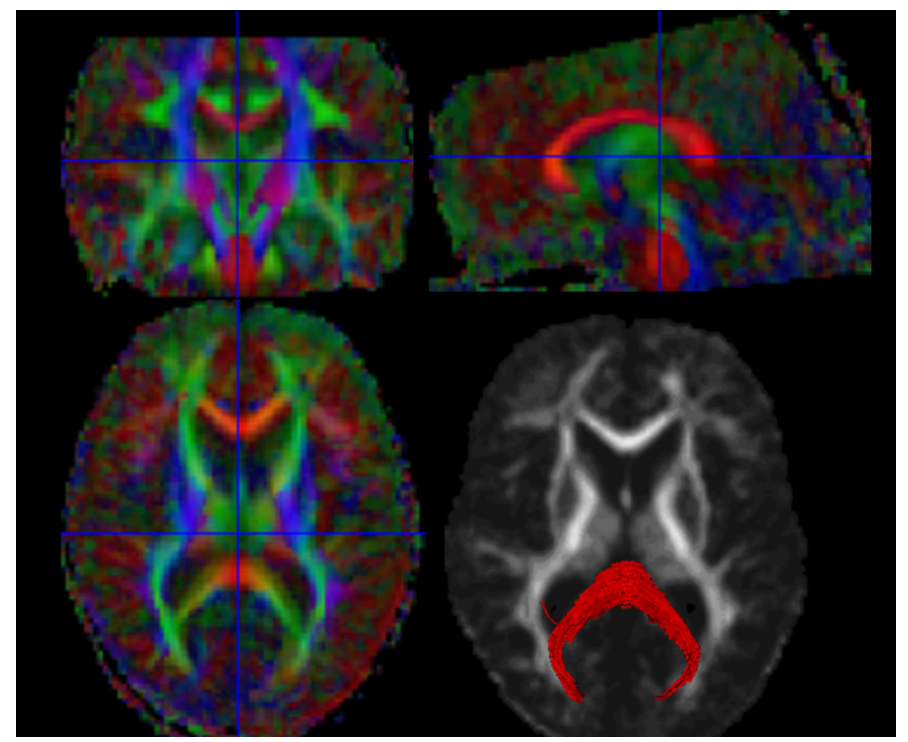

Figure 1.3 Diffusion tensor color map of the averaged brains of 6 medulloblastoma survivors. The colors indicate the principal direction of diffusion in the voxel. Green is anterior to posterior, Blue inferior to superior, and Red is left to right. (Lower Right) A computer aided tractogram is overlaid onto an FA map.

injury in the mouse model over a one to 4 week period [18]. Histological correlates verified that DTI could detect pure axonal injury without gliosis or demylination, as seenin the first 24 hours of injury, as well as being able to observe the later stages of axonal injury often marked by pronounced gliosis. They further demonstrated that DTI was more sensitive to injury than conventional MRI. These studies were further supported by Budde, et al. (2008), who demonstrated that in vivo, DTI measures correlated with neurological impairment in mouse models with Multiple Sclerosis.

Finally, several studies have shown that DTI is useful in the evaluation of cognitive and neuropsychological measures. In a study of dyslexic adults, Klingberg, et al. (2000) found that reading ability in both reading-disabled adults and those with normal reading ability was positively correlated with the FA of water diffusion in a perisylvian area in the left hemisphere [19]. In an attempt to extend Klingberg's findings, Deutsch, et al. (2005), reported that the presence of FA values at early ages correlated to with multiple aspects of reading performance, such as word reading, spelling and rapid naming. Additionally, Schmithorst, et al. (2007), found positive correlation of FA in the left temporal-parietal with higher reading skills and general cognitive ability [20]. Schmithorst and Klingberg's results further agree with Wilke, et al. (2003), which found correlations in brain structure and intelligence with voxel-based morphometry measures in normal pediatric populations [19-21]. All of these studies provide credibility that FA is a robust indicator of underlying white matter organization and changes in FA correlate with reading ability. 


\subsection{Specific Aims}

The work presented in this dissertation represents a program of research using Diffusion Tensor Imaging to evaluate, diagnose and treat pediatric brain tumor patients. I hypothesised that DTI would improve our ability to detect disease involvement in white matter pathways. I hypothesised DTI would impact treatment which may alter the course of disease progression. Finally, I hypothesised that DTI would improve our understanding of how treatment affects cognitive and functional outcomes in pediatric brain tumor survivors. To test these hypotheses, I defined the following specific aims;

1) Validate that invasion of corticospinal, transverse pontine and medial lemniscal tracts by pontine brain stem gliomas can be demonstrated by DTI and show that reduced FA and elevated ADC values correlate with the degree of tract invasion and neurologic deficits.

2) Establish the value of performing DTI and tractography in children with brainstem tumors to demonstrate evidence of axonal degeneration in pediatric patients with brainstem tumors, with possible implications for surgical planning.

3) Establish the feasibility of using DT imaging for preoperative visualization of the white matter tracts of the brainstem in relation to focal low-grade gliomas, and describe these findings in terms of the Epstein and Farmer hypothesis for characteristic growth patterns of low-grade gliomas.

4) Demonstrate the use of DTI to investigate functional anatomy and connectivity in the extrastriate ventral visual pathways and to characterize the relationship in regards to acquired reading deficits and therapy-induced brain damage in pediatric medulloblastoma survivors.

5) Demonstrate the utility of DTI to describe the disrupted physiology underlying the striking neurobehavioral deficits associated with Cerebellar Mutisim.

\subsection{Organization}

In Chapter 2, I will discuss the methods used to derive and analyze the diffusion tensor image experiments outlined in the remainder of this dissertation. Chapter 3 is the first of a series five translational studies that demonstrate the utility of DTI in pediatric brain tumor population. Specifically, Chapter 3 will discuss the use of DTI in the evaluation of brainstem gliomas in pediatric patients. Prior to this study little if any research had been done using DTI to visualize the brainstem. Chapter 4 will discuss how I used DTI to investigate the pathophysiological effects of Brainstem tumors on neighboring white matter tracts. This study will demonstrate how I used DTI to measure axonal degeneration in the motor and sensory pathways. Chapter 5 will discuss how DTI can be used to improve surgical morbidities associated with resection of brainstem tumors. This chapter will demonstrate how improved tractography techniques improved the outcomes of specific surgical cases. Chapter 6 will discuss how I used DTI to investigate neurocognitive sequelae of medulloblastoma therapy. I demonstrate how new 
novel techniques in DTI measures can be used to determine the underlying neural networks damaged by treatments and hopefully can be used to refine treatment regimens. Chapter 7 will discuss how DTI can be used to investigate neurological conditions with unknown etiologies and how these results can open up new avenues of neurocognitive research. Finally, Chapter 8 will summarize all of these studies and discuss how DTI has improved diagnosis, treatment and reduction of morbidities associated with cancer treatment. We will also discuss future avenues of research that these studies have outlined. 


\section{Chapter 2. Analysis Methods}

\subsection{Introduction}

In the previous chapter, I discussed the mathematical basis for the diffusion signal and its measurements with Magnetic Resonance Imaging (MRI). We also outlined a general description of the 3-D tensor and described the calculations of eigenvectors and eigenvalues. In this chapter, I will discuss how the tensor is calculated from the Diffusion Weighted Images (DWI) in six or more directions. I will focus on two well published methods. The first is an exact analytical method for exactly six directions while the second method, using linear least squares, allows for more than six directions and provides the user with a better way to deal with noise present in low signal to noise ratio images. We will also discuss the relative contribution of noise and uncertainty in using these methods and outline methods used to estimate the variance in the derived parameters from the experimental DTI data using bootstrap analysis. Finally, we will complete our discussion on DTI analysis by explaining and comparing two analysis methods used to evaluate the DTI data described in the remainder of this dissertation.

\subsection{Analytical Solution}

As previously discussed, the diffusion tensor can be calculated from diffusion weighted data collected with six or more non-collinear diffusion sensitizing gradient directions. In the special case in which there are exactly six directions, the measured diffusion coefficients can be written as:

$$
\begin{aligned}
& D_{i}=b\left(g_{x i}^{2} D_{x x}+g_{y i}^{2} D_{y y}+g_{z i}^{2} D_{z z}+2 g_{x i} D_{x y}+\right. \\
& \left.2 g_{x i} g_{z i} D_{x z}+2 g_{y i} g_{z i} D_{y z}\right)
\end{aligned}
$$

Where $g_{x i}, g_{y i}$, and $g_{z i}$ are the normalized gradient components and $b$ describes the gradient duration and strength. These six equations in six unknown can be solved as a system of linear equations with such techniques as Cramer's rule.

For the studies discussed in Chapter 3 and 4 of this dissertation, we modified the diffusion calculation routine published by Russ Poldrack for Statistical Parametric Mapping (SPM) 99 (Appendix A). SPM is a free open source software package developed by the Wellcome Trust Center for Neuroimaging at the University College of London. The SPM suite includes tools for spatial processing, statistical modeling, diffusion image processing, and graphical representation.

The method requires we represent the six distinct tensor elements and the logarithm of the $b=0\left(\mathrm{~S}_{0}\right)$ signal intensity image as a seven element column vector $(\alpha)$. 


$$
\alpha=\left[D_{x x}, D_{y y}, D_{z z}, D_{x y}, D_{y z}, \ln \left(S_{0}\right)\right]^{T}
$$

Each individual $\mathbf{b}$ matrix is represented by a six element row vector and is a part of a seven element row vector $\mathbf{B}$.

$$
\begin{aligned}
& b_{i}=\left(b_{x x i}, b_{y y i}, b_{z z i}, 2 b_{x y i}, 2 b_{x z i}, 2 b_{y z i}\right) \\
& B_{i}=\left(-b_{x x i},-b_{y y i},-b_{z z i},-2 b_{x y i},-2 b_{x z i},-2_{y z i}, 1\right)
\end{aligned}
$$

These row vectors are combined into one large N x 7 B matrix. And the logarithm of the predicted signal intensities are given by an $\mathrm{N} x 1$ column vectors $\xi_{\mathrm{i}}$.

$$
\begin{aligned}
\xi_{i}= & B_{i} \alpha=\ln \left(S_{i}\right)=\ln \left(S_{0}\right)-b_{i}: D \\
\xi_{i}= & -b_{x x i} D_{x x}-b_{y y i} D_{y y}-b_{z z i} D_{z z}-2 b_{x y i} D_{x y} \\
& -2 b_{x z i} D_{x z}-2 b_{y z i} D_{y z}+\ln \left(S_{0}\right)
\end{aligned}
$$

For this method, data are collected in each gradient direction and in each corresponding negative direction. These cross-terms are then eliminated by taking the geometric mean of the signal intensities of each pair and the ADC's calculated. These cross-terms are in practice not completely eliminated, but the residual error is much less than the noise associated with the low SNR diffusion weighted images [22]. In the Poldrack method (Eq 2.7), the raw diffusion images are expressed as the logarithm of the individual signal intensities. The apparent diffusion coefficients are subsequently solved as follows:

$$
\begin{aligned}
& D_{x x}=- 1 /(4 * b)\left\{\left(\log D W I_{x z}\right)\right)+\left(\log D W I_{-x z}\right)-\left(\log D W I_{y z}\right)-\left(\log D W I_{-y z}\right) \\
&\left.+\left(\log D W I_{x y}\right)+\left(\log D W I_{-x y}\right)-2 *\left(\log D W I_{l o w b}\right)\right\} \\
& D_{x y}=-1 /(4 * b)\left\{\left(\log D W I_{x y}\right)-\left(\log D W I_{-x y}\right)\right\}(\operatorname{Eq} 2.7) \\
& D_{x z}=-1 /(4 * b)\left\{\left(\log D W I_{x z}\right)+\left(\log D W I_{-x z}\right)\right\} \\
& D_{y y}=-1 /(4 * b)\left\{\left(-\log D W I_{x z}\right)-\left(\log D W I_{-x z}\right)+\left(\log D W I_{y z}\right)+\left(\log D W I_{-y z}\right)\right. \\
&\left.\quad+\left(\log D W I_{x y}\right)+\left(\log D W I_{-x y}\right)-\left(2^{*} \log D W I_{l o w b}\right)\right\} \\
& D_{y z}=-1 /(4 * b)\left\{\left(\log D W I_{y z}\right)-\left(\log D W I_{-y z}\right)\right. \\
& D_{z z}=-1 /(4 * b)\left\{\left(\log D W I_{x z}\right)+\left(\log D W I_{-x z}\right)+\left(\log D W I_{y z}\right)+\left(\log D W I_{-y z}\right)\right. \\
&\left.-\left(\log D W I_{x y}\right)-\left(\log D W I_{-x y}\right)-2 *\left(\log D W I_{l o w b}\right)\right\}
\end{aligned}
$$


The solutions from these equations yield the 6 diffusion terms for the diffusion tensor calculations outlined in Chapter 1.

It should be noted that the observed data contains some element of noise from both random and systemic deviation. Ideally, noise should be eliminated from the data prior to calculation of the tensor derivatives. However, in practice, noise cannot be differentiated in low signal-to-noise ratio (SNR) images. To describe the effects of noise on the diffusion tensor we express noise as a vector ' $\eta$ ' for the noisy data in each acquisition. Transforming Eq 2.5, we see the overall contribution of noise to the measures is expressed as;

$$
\xi=-b_{i}: D+\eta
$$

Noise causes a positive bias in the DTI data $[23,24]$. This noise produces errors of uncertainty in the derived parameters, and cannot be completely described by conventional propagation of error formulas [22]. However, with exactly six gradient directions, if repetitions are averaged before the tensor is calculated, errors in the tensor elements and derived parameters will be minimized because the $b=0$ image are combined prior to calculating the tensor [22].

\subsection{Linear Least Squares Fit}

A more complete method for dealing with noise requires that the individual measured data be treated equally in calculating the tensor. This corresponds to an unweighted linear least fit square fit of the signal intensity (Eq 2.9). The pseudo inverse is then calculated by singular value decomposition which yields the corresponding linear least-squares fit of the logarithms of the signal intensities (Eq 2.10). In general, each individual signal data point is equally accurate and this method reliable, however, the same does not hold for the logarithms. For example, if the variance $\left(\sigma_{i}\right)$ of the signal intensities is equal to the uncertainty in $\ln \left(\mathrm{S}_{\mathrm{i}}\right)$ then it is proportional to $1 / \mathrm{S}_{\mathrm{i}}$. Thus, the least squares fit of the logarithm signal intensities will be more weighted for high $\ln \left(\mathrm{S}_{\mathrm{i}}\right)$ values than of lower $\ln \left(\mathrm{S}_{\mathrm{i}}\right)$ values [22].

$$
\begin{aligned}
& \left(B^{T} B\right)^{-1} B^{T} B \alpha=\alpha=\left(B^{T} B\right)^{-1} B^{T} x \\
& \left(B^{T} B\right)^{-1} B^{T}=B^{\Psi}
\end{aligned}
$$

To deal with this problem, we must weight the contribution of the high and low $\mathrm{S}_{\mathrm{i}}$ values. We do this by adding diagonal $\mathrm{N} x \mathrm{~N}$ matrix of $S_{i}^{2} / \sigma_{i}^{2}$ elements to Eq 2.9.

$$
\Sigma^{-1}=\operatorname{diag}\left(\frac{S_{i}^{2}}{\sigma_{i}^{2}}\right)
$$


This yield's the covariance matrix:

$$
\alpha=\left(B^{T} \Sigma^{-1} B\right)^{-1}\left(B^{T} \Sigma^{-1}\right) x
$$

In theory, each original image should have the same noise level and therefore $\sigma_{\mathrm{i}}$ is the same for each image. In practice, noise is not stochastic and the $\sigma_{\mathrm{i}}$ in the $\Sigma^{-1}$ matrix must be modified to reflect the differing amounts of signal averaging (Eq 2.13). This is accomplished by first calculating a linear least-square fit on the noisy $\mathrm{S}_{\mathrm{i}}$ data and then repeating the calculations with the fitted $\mathrm{S}_{\mathrm{i}}$. This yields a value closer to the original data than the noisy data [22].

$$
B^{T} \Sigma^{-1} B=\left[\begin{array}{ccccccc}
\sum_{i=1}^{N} b_{x x i}^{2} \frac{S_{i}^{2}}{\sigma_{i}^{2}} & \sum_{i=1}^{N} b_{x x i} b_{y y i} \frac{S_{i}^{2}}{\sigma_{i}^{2}} & \ldots & 2 \sum_{i=1}^{N} b_{x x} b_{y z i} \frac{S_{i}^{2}}{\sigma_{i}^{2}} & -\sum_{i=1}^{N} b_{x x i} \frac{S_{i}^{2}}{\sigma_{i}^{2}} \\
\sum_{i=1}^{N} b_{y y} b_{x x i} \frac{S_{i}^{2}}{\sigma_{i}^{2}} & \sum_{i=1}^{N} b_{y y i}^{2} \frac{S_{i}^{2}}{\sigma_{i}^{2}} & \ldots & 2 \sum_{i=1}^{N} b_{y y} b_{y z i} \frac{S_{i}^{2}}{\sigma_{i}^{2}} & -\sum_{i=1}^{N} b_{y y i} \frac{S_{i}^{2}}{\sigma_{i}^{2}} \\
\sum_{i=1}^{N} b_{z z} b_{x x i} \frac{S_{i}^{2}}{\sigma_{i}^{2}} & \sum_{i=1}^{N} b_{z z i} b_{y y i} \frac{S_{i}^{2}}{\sigma_{i}^{2}} & \ldots & 2 \sum_{i=1}^{N} b_{z z i} b_{y z i} \frac{S_{i}^{2}}{\sigma_{i}^{2}} & -\sum_{i=1}^{N} b_{z z i} \frac{S_{i}^{2}}{\sigma_{i}^{2}} \\
2 \sum_{i=1}^{N} b_{x y} b_{x x i} \frac{S_{i}^{2}}{\sigma_{i}^{2}} & 2 \sum_{i=1}^{N} b_{x y} b_{y y i} \frac{S_{i}^{2}}{\sigma_{i}^{2}} & \ldots & 4 \sum_{i=1}^{N} b_{x y} b_{y z i} \frac{S_{i}^{2}}{\sigma_{i}^{2}} & -2 \sum_{i=1}^{N} b_{x y i} \frac{S_{i}^{2}}{\sigma_{i}^{2}} \\
2 \sum_{i=1}^{N} b_{x z i} b_{x x i} \frac{S_{i}^{2}}{\sigma_{i}^{2}} & 2 \sum_{i=1}^{N} b_{x z} b_{y y i} \frac{S_{i}^{2}}{\sigma_{i}^{2}} & \ldots & 4 \sum_{i=1}^{N} b_{x z} b_{y z i} \frac{S_{i}^{2}}{\sigma_{i}^{2}} & -2 \sum_{i=1}^{N} b_{x z i} \frac{S_{i}^{2}}{\sigma_{i}^{2}} \\
2 \sum_{i=1}^{N} b_{y z l} b_{b x x i} \frac{S_{i}^{2}}{\sigma_{i}^{2}} & 2 \sum_{i=1}^{N} b_{y z b} b_{y y i} \frac{S_{i}^{2}}{\sigma_{i}^{2}} & \ldots & 4 \sum_{i=1}^{N} b_{y z i}^{2} \frac{S_{i}^{2}}{\sigma_{i}^{2}} & -2 \sum_{i=1}^{N} b_{y z i} \frac{S_{i}^{2}}{\sigma_{i}^{2}} \\
-\sum_{i=1}^{N} b_{x x i} \frac{S_{i}^{2}}{\sigma_{i}^{2}} & -\sum_{i=1}^{N} b_{y y i} \frac{S_{i}^{2}}{\sigma_{i}^{2}} & \ldots & -2 \sum_{i=1}^{N} b_{y z i} \frac{S_{i}^{2}}{\sigma_{i}^{2}} & -\sum_{i=1}^{N} \frac{S_{i}^{2}}{\sigma_{i}^{2}}
\end{array}\right]
$$

The benefit of this method derives from the fact that the estimate of the $\ln \left(\mathrm{S}_{0}\right)$ is produced by the least-squares fit, while the analytic method averages the $b=0$ signal intensities. Additionally, this method allows for more than 6 gradient directions and/or two or more $b$ factors. And a final advantage is that the covariance matrix can be used to make propagation of errors calculations like those used in Bootstrap analysis.

Implementation of this method for this dissertation was performed by developing in-house modifications of the Volkmar Gaucher DTI processing algorithms originally developed for SPM2. Later, I developed methods and software to implement the DTIfit algorithm developed for FSL (Appendix B, and C). My modifications resolved Siemens' DICOM compatibility issues and decreased processing time by incorporating batch processing and employing a parallel processing algorithm on a 128 node distributed computing network (Appendix D and E). 


\section{$2.4 \quad$ Fiber Tracking}

To investigate the white matter fiber connections in pediatric cancer patient's we evaluated probabilistic tracking and streamline tracking in random samples of patient and volunteer data. Probabilistic tracking was implemented with tools from the FSL software package (Oxford Centre for Functional Magnetic Resonance in the Brain, www.fmrib.ox.ac.uk/fsl). For each set of DTI images a Bayesian Estimation of Diffusion Parameters Obtained using Sampling Techniques (BEDPOST) was generated. The BEDPOST procedure uses a Markov Chain Monte Carlo (MCMC) sampling technique to generate sampling distribution of the diffusion profiles for each voxel. These distributions are then sampled along a voxel-wise principle diffusion direction to generate a probabilistic streamline. Multiple samples of the distribution map allowed me to produce a connectivity distribution for a single seed point. Additionally, this technique can be used to quantify connectivity values between a seed mask and several user defined target masks. The probabilistic tracking output will be used to identify the commissural and ventral visual association fibers for region of interest (ROI) analysis. To improve the utility of this application in the clinical setting, I implemented a parallelized BEDPOST estimation on the 280-cpu IBM BladeServer Linux cluster located in the high performance computing facility at St. Jude Children's Research Hospital. This reduced processing time from 20 hours to less than 20 minutes (Appendix E).

Concurrently, streamline tracking was performed using public domain software (http://www.ia.unc.edu/dev/download/fibertracking/index.htm). This algorithm was first proposed by Mori and $\mathrm{Xu}$, and is based on the extraction of the Principal Diffusion Direction (PDD) of the tensor in each voxel. In general, a PDD is determined for each voxel in the image, giving a vector field of principal diffusion. Starting from a seed point, tracking progresses along the PDD until the diffusion is no longer linear $(\mathrm{FA}<$ $0.17)$. This procedure is applied to every voxel in the target region. Then the reconstructed paths are examined to determine which paths pass through the seed point. Only those fibers that pass through the seed point are retained. For branching fiber tracks, this assures that all possible branches are tracked. To reduce susceptibility to noise, tracking proceeds based on local connectivity rather than a rigid voxel-based grid system. For example, each new connection is judged on its "transition smoothness" (C) (Eq 2.14). A threshold is set for the amount of fiber curvature allowed between transitions. Additionally, local connectivity is quantified in a "statistical sense" (R), so that the tracking terminates if the tracking deviates severely from the connectivity of the neighborhood (Eq 2.15) (http://www.cs.unc.edu/ fillard/).

$$
\begin{aligned}
& C=\left|\overrightarrow{u_{l}} \bullet \overrightarrow{u_{l-1}}\right| \\
& R=\frac{2}{s(s-1)} \bullet \sum_{i=o}^{s-2} \sum_{j=i+1}^{s-1}\left|\overrightarrow{u_{i}} \bullet \overrightarrow{u_{j}}\right|
\end{aligned}
$$


Measures of total fibers tracked and distance tracked were used to evaluate performance. Fiber tracks were also compared to cadaver based anatomic references to determine if tracks were anatomically accurate.

\subsection{Tract Based Spatial Statistics}

I employed Tract Based Spatial Statistics (TBSS) in my translational studies to investigate global differences in FA in the medulloblastoma patient populations (Chapters 6 and 7) [28]. The strength of this analysis lies in its ability to conduct cross subject voxel wise comparisons that are alignment invariant. It also improves on the fibertracking method, because it does not require manual intervention to specify a tract of interest.

The basic approach (outlined in Fig 2.1) includes first aligning the native FA images of all subjects into a standard brain space using a combined global correction and local correction transformation (Eq 2.16).

$$
T(x, y, z)=T_{\text {global }}(x, y, z)+T_{\text {local }}(x, y, z)
$$

The global transformation is accomplished using 6 degrees of freedom rigid transformation (Eq 2.17). These six parameters characterize translation in the $\mathrm{x}, \mathrm{y}$ and $\mathrm{z}$ directions and rotations around the $\mathrm{x}, \mathrm{y}$ and $\mathrm{z}$ axis. Once these transformation parameters have been determined, the images are resampled producing a realigned image.

$$
T_{\text {global }}(x, y, z)=\left(\begin{array}{lll}
\theta_{11} & \theta_{12} & \theta_{13} \\
\theta_{21} & \theta_{22} & \theta_{23} \\
\theta_{31} & \theta_{32} & \theta_{33}
\end{array}\right)\left(\begin{array}{l}
x \\
y \\
z
\end{array}\right)+\left(\begin{array}{c}
\theta_{14} \\
\theta_{24} \\
\theta_{34}
\end{array}\right)
$$

This affine transformation produces only grossly aligned images. Local differences require a more meticulous model for image registration. The method implemented by TBSS uses an approach based on Free-Form Deformation (FFD) and Bsplines [25]. The basic idea is to deform an image by moving control points of an underlying mesh to minimize the registration cost function (Eq 2.20) [26]. The FreeForm Deformation is best described as a 3-D tensor product of a 1-D cubic B-spline (Eq 2.18).

$$
T_{\text {local }}(x, y, z)=\sum_{l=0}^{3} \sum_{m=o}^{3} \sum_{n=0}^{3} B_{l}(u) B_{m}(v) B_{n}(w) \varphi_{i+1, j+m, k+n}
$$

Where $\varphi_{\mathrm{i}, \mathrm{j}, \mathrm{k}}$ denotes a $n_{x} * n_{y} * n_{z}$ mesh of control points with uniform spacing and $i=$ $\left[x / n_{x}\right]-1, j=\left[y / n_{y}\right]-1, k=\left[z / n_{z}\right]-1, u=x / n_{x}-\left[x / n_{x}\right], v=y / n_{y}-\left[y / n_{y}\right], w=z / n_{z}-\left[z / n_{z}\right]$ and where $\mathrm{B}_{1}$ represents the $\mathrm{I}^{\text {th }}$ basis function of the B-spline [26] (Eq 2.19). 


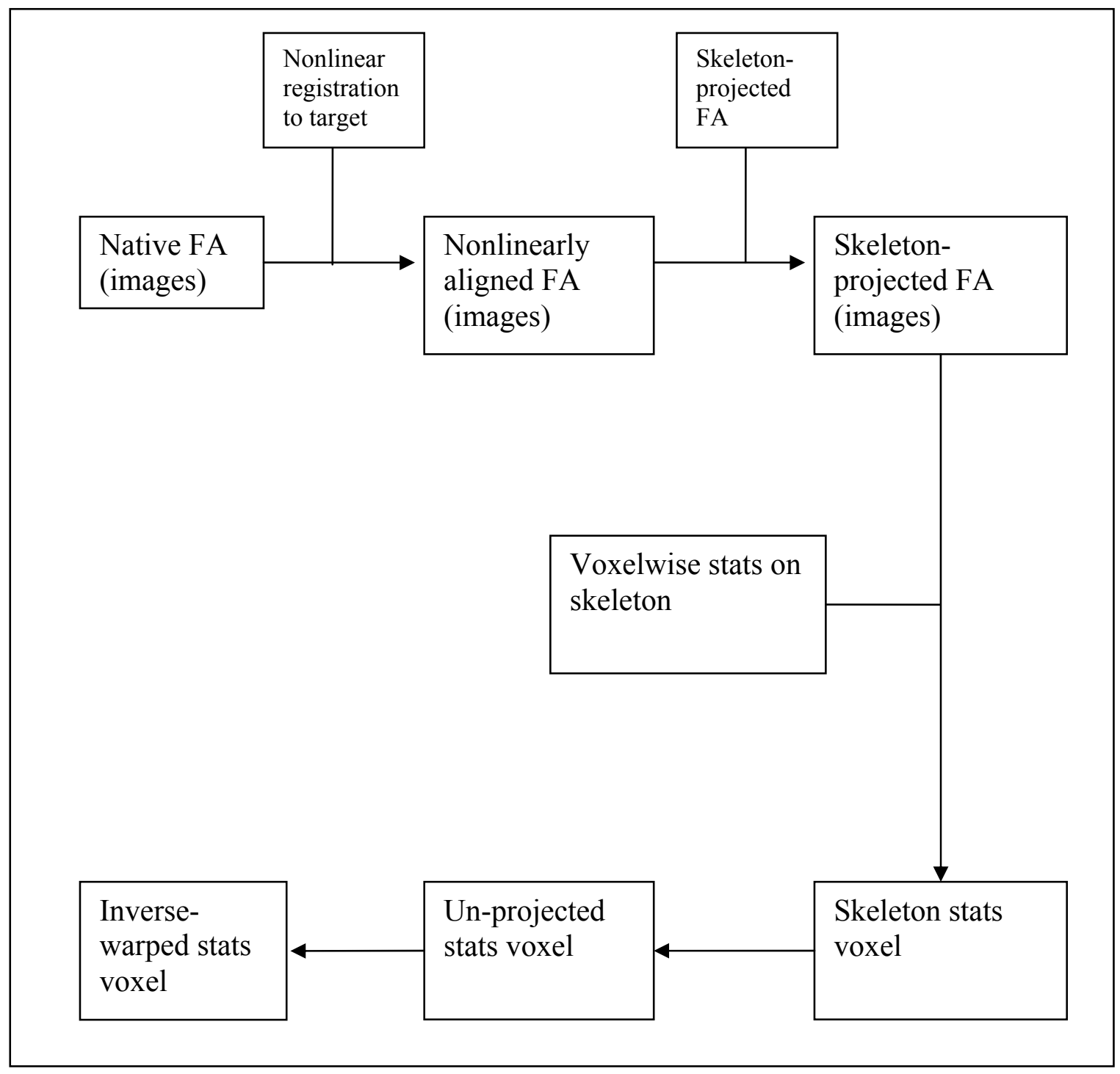

Figure 2.1 Outline of the general procedure for performing Tract-Based Spatial Statistics (TBSS). 


$$
\begin{aligned}
& B_{0}(u)=(1-u)^{3} / 6 \\
& B_{1}(u)=\left(3 u^{3}-6 u^{2}+4\right) / 6 \\
& B_{2}(u)=\left(-3 u^{3}+3 u^{2}+3 u+1\right) / 6 \\
& B_{3}(u)=u^{3} / 6
\end{aligned}
$$

Changing the control points only affects the local neighborhood and does not affect the global rigid deformation. The optimal transformation minimizes the cost function associated with both the global $(\Theta)$ and local transformation parameters $(\Phi)$.

$$
C(\Theta, \Phi)=-C_{\text {simularity }}\left(I\left(t_{0}\right), T(I(t))\right)+\lambda C_{\text {smooth }}(T)
$$

The $\lambda$ term describes the trade off between the alignment of two image volumes, the $C_{\text {simularity }}$ term represents the cost associated with the image similarity and the $C_{\text {smooth }}$ is the cost associated with the smoothness transformation.

$$
\begin{aligned}
C_{\text {smooth }} & =\frac{1}{V} \int_{0}^{x} \int_{0}^{x} \int_{0}^{z}\left[\left(\frac{\partial^{2} T}{\partial x^{2}}\right)^{2}+\left(\frac{\partial^{2} T}{\partial y^{2}}\right)^{2}+\left(\frac{\partial^{2} T}{\partial z^{2}}\right)+2\left(\frac{\partial^{2} T}{\partial x y}\right)^{2}+\left(\frac{\partial^{2} T}{\partial x z}\right)^{2}\right. \\
& \left.+2\left(\frac{\partial^{2} T}{\partial y z}\right)^{2}\right] d x d y d z
\end{aligned}
$$

The similarity cost function is defined as the amount of information one image A contains about the second image B. To avoid any dependency TBSS uses normalized mutual information as its measure of image alignment [26]. If both images are aligned, the mutual information is maximized.

$$
C_{\text {simularity }}=(A, B)=\frac{H(A)+H(B)}{H(A, B)}
$$

Next the mean aligned FA images for all subjects is created and then used to create a mean FA skeleton that represents the centers of the major tracts of all subjects. Generally this is accomplished by searching along all voxels in a direction perpendicular to the tract direction and finding the voxel with the highest FA. The contiguous sets of maximal FA values found in this way are connected to represent the tract skeleton.

To achieve this, I first estimated the local surface perpendicular direction. Because FA values decline as you move out of the tract into the surrounding grey matter, FA values will be higher on one side of the voxel than on the other. Exploiting this fact, we can take the first derivative of the FA image and find the vector from the current voxel to the local maxima (within a $3 \times 3 \times 3$ voxel neighborhood). This should point in the direction perpendicular to the tract center. This method fails at voxels that lie close to (within $0.1 \mathrm{~mm}$ ) of the local maxima [27]. 
To determine the perpendicular in the case of local maxima lying close to the current voxel, an alternative method is used. The second derivative is used to determine the direction of local maximal change in a $3 \times 3 \times 3$ neighborhood. In essence, the mean of an opposing pair of voxels is subtracted from the center value and the direction in which the value is maximized is assumed to be the direction perpendicular to the tract [27].

Once the local perpendicular directions are estimated, the search for the tract skeleton can be begun. The FA values of neighboring voxels perpendicular to the local tract surface are compared. The FA with the maximal value is then labeled as the center of the tract and marked as lying on the skeleton. This is performed at all voxels were the FA is above a threshold value determined subjectively. This eliminates unnecessary processing and restricts analysis to voxels within white matter.

Each subjects aligned FA image is then projected on the mean FA skeleton. In this way, the projected FA value is taken from the centers of the tracts in the original FA [28]. This also allows for residual misalignment between subjects to be corrected. To do this, each aligned FA image is searched for the maximum FA value nearest the projected skeleton in a direction perpendicular to the skeletal tract. The maximum FA value is then mapped to the skeleton voxel. "Thus any systemic differences in the exact location between groups of subjects will thus not bias the comparison FA values between groups"[27,28]. Finally, voxelwise statistics across subjects on the skeletonized FA is conducted.

\subsection{Summary}

In this chapter, I discussed the implementation of two methods to calculate the diffusion tensor from the diffusion weighted images. I compared and contrasted these methods with respect to their relative treatment of noise and propagation of errors in the calculated tensors and demonstrated the relative strengths and weakness of each method. I also discussed my contributions to the implementation of these methods in our series of translational studies. Finally, I presented two methods used to analyze the diffusion tensor measures and determine white matter integrity, namely Fiber Tracking and Tract Based Spatial Statistics.

In the following chapters, the reader will see how these techniques were applied to a series of translational trials. Each study had its own unique set of criteria and restrictions for the choice of each of the above outlined methods, so that no one method was chosen for all of the following studies. To facilitate an objective and straight forward presentation of each study, I will simply summarize the technique used for each study in the methods and materials sections and allow the reader focus on the resulting data with the appreciation of the limitations and strengths of those methods outlined in this chapter. 


\section{Chapter 3. DTI of Brainstem Gliomas}

\subsection{Introduction and Overview}

Diffuse brainstem gliomas (BSGs) comprise a group of malignant tumors with a much poorer prognosis than those of focal brainstem tumors. BSGs account for about $15 \%$ of pediatric brain tumors and comprise approximately $20 \%$ to $30 \%$ of posterior fossa tumors $[29,30]$. Histopathologically, these diffuse pontine tumors are usually differentiated WHO grade II fibrillary astrocytomas or WHO grade III anaplastic astrocytomas at diagnosis and are known to infiltrate between normal axonal fibers [30]. Although the appearance of focal brainstem tumors and that of diffuse brainstem tumors typically differ by conventional magnetic resonance imaging (MRI), distinguishing focal from diffuse involvement is sometimes imprecise. The discovery of a method that distinguishes between these 2 types of tumors is valuable, as the treatment and prognosis for the brainstem tumors are substantially different [31]. On MRI T2W images, focal tumors are typically well marginated, often enhance, may be exophytic, and occupy less than $50 \%$ of the axial diameter of the brainstem, whereas diffuse tumors are poorly marginated, rarely enhance, occupy more than $50 \%$ of the axial diameter of the brainstem, lack an exophytic component, and commonly engulf the basilar artery [29].

Conventional MRI has demonstrated prognostic value in treatment of brainstem tumors [30], but white matter appears homogenous on MRIs; therefore, this method cannot precisely define essential aspects of tract location, displacement, or invasion. In contrast, diffusion tensor imaging (DTI) detects anisotropic diffusion, thereby allowing the visualization of major fiber tracts in the brainstem. Thus, DTI may provide information about tumor involvement in white matter tracts. Recent work on the application of DTI in supratentorial tumors has explored quantitative values of the apparent diffusion coefficient (ADC) and fractional anisotropy (FA) to characterize tumors [32-35], peritumoral involvement of tract [36,37], and the appearance of tumoraltered white matter tracts [38]. On the basis of these studies, DTI has become an important component of preoperative evaluation and treatment planning for patients with supratentorial tumors in certain institutions [36].

Current management of suspected focal lesions in the brainstem involves stereotactic biopsy or resection [30], depending on the location and size of the tumor. Surgical options have an acceptable but real risk [39] and are essential in determining therapy for focal brainstem lesions. The use of DTI to assess tumor involvement of brainstem tracts or to assist surgical planning for such cases has not been reported.

We report a study in which we tested the hypothesis that invasion of corticospinal, transverse pontine and medial lemniscal tracts by pontine BSGs can be demonstrated by DTI data showing reduced FA and elevated ADC values. Additionally, we evaluated the correlation between the degree of tract invasion and neurologic deficits. 


\subsection{Patient Demographics}

DTI data were analyzed in a retrospective study of 3 groups. The experimental group consisted of 6 patients with diffuse BSGs and 1 with a focal brainstem tumor. The patient group's mean age was 7.7 years (range, $6-15$ years). The second group consisted of 4 patients with cancer who served as patient controls. The mean age of this group was 16.5 years (range, 12-20 years), and the sites and types of primary tumor were prostatic rhabdomyosarcoma, noncranial metastatic melanoma, pineal glioma, and parafalcine Rosai-Dorfman histiocytic lesion. Patients from these 2 groups underwent MRI and DTI imaging procedures at St. Jude during 2002-2003. The third group consisted of 5 healthy adult controls that were nonsymptomatic graduate students and MRI technicians whose ages ranged from 21 to 37 years. Clinical data were obtained by chart review, as approved by the institutional review board (IRB).

\subsection{Conventional Magnetic Resonance Imaging}

Magnetic resonance imaging was performed on a 1.5-Tesla Siemens Symphony scanner using a quadrature head coil. Conventional imaging included the following: FLASH T1W TR 165-218 msec, TE $4 \mathrm{msec}$, FOV $158 \times 210$, slice thickness $5 \mathrm{~mm} / 0 \mathrm{~mm}$ gap, matrix $256 \times 256,2 \mathrm{NEX}$ in the sagittal, axial and coronal planes; axial double echo T2W TR 4000-6000 msec, TE $16-109 \mathrm{msec}$, FOV $210 \times 210$, slice thickness $5 \mathrm{~mm} / 0$ gap, matrix 512 ×512, 2 NEX; and axial FLAIR TR 9000-10,000 msec, TE $112 \mathrm{msec}$, TI 2400 , FOV $158 \times 210$, slice thickness $5 \mathrm{~mm} / 0$ gap, matrix $144 \times 256$, 2 NEX.

\subsection{Diffusion Tensor Imaging}

Diffusion-weighted, echo-planar images were acquired with a double spin-echo sequence (Reese, et al. 2003) [40] $(230 \mathrm{~mm} \times 230 \mathrm{~mm} \times 200 \mathrm{~mm}$ FOV, TR 10,000 $\mathrm{msec}$, TE $100 \mathrm{msec}, 4$ acquisitions per series). Diffusion encoding was applied along 6 noncollinear directions $(b=1000)$, and 1 image was acquired without diffusion encoding. Image acquisitions were realigned using the realignment tools within Statistical Parametric Mapping (SPM99) (Wellcome Institute of Neurology, London, UK). Diffusion tensors were calculated using the SPM diffusion toolbox software developed for SPM99; this software can be accessed at http://sourceforge.net/projects/spm-toolbox/ [41]. FA, ADC, and eigenvector maps were calculated (Fig 3.1, 3.2). To aid in the visualization of the fiber tracts, we used an RGB-orientation color map to demonstrate fiber shape and direction [42]. FA and ADC were evaluated in 6 regions of interest (ROIs) hand drawn under the supervision of experienced neuroradiologists (K.J.H., J.W.L.). The ROIs were drawn around the bilateral corticospinal tracts, transverse pontine fibers, and medial lemnisci at the level of the middle cerebellar peduncles. Analyses of the DTI data were performed blind to the results of the patient's neurologic evaluation. 


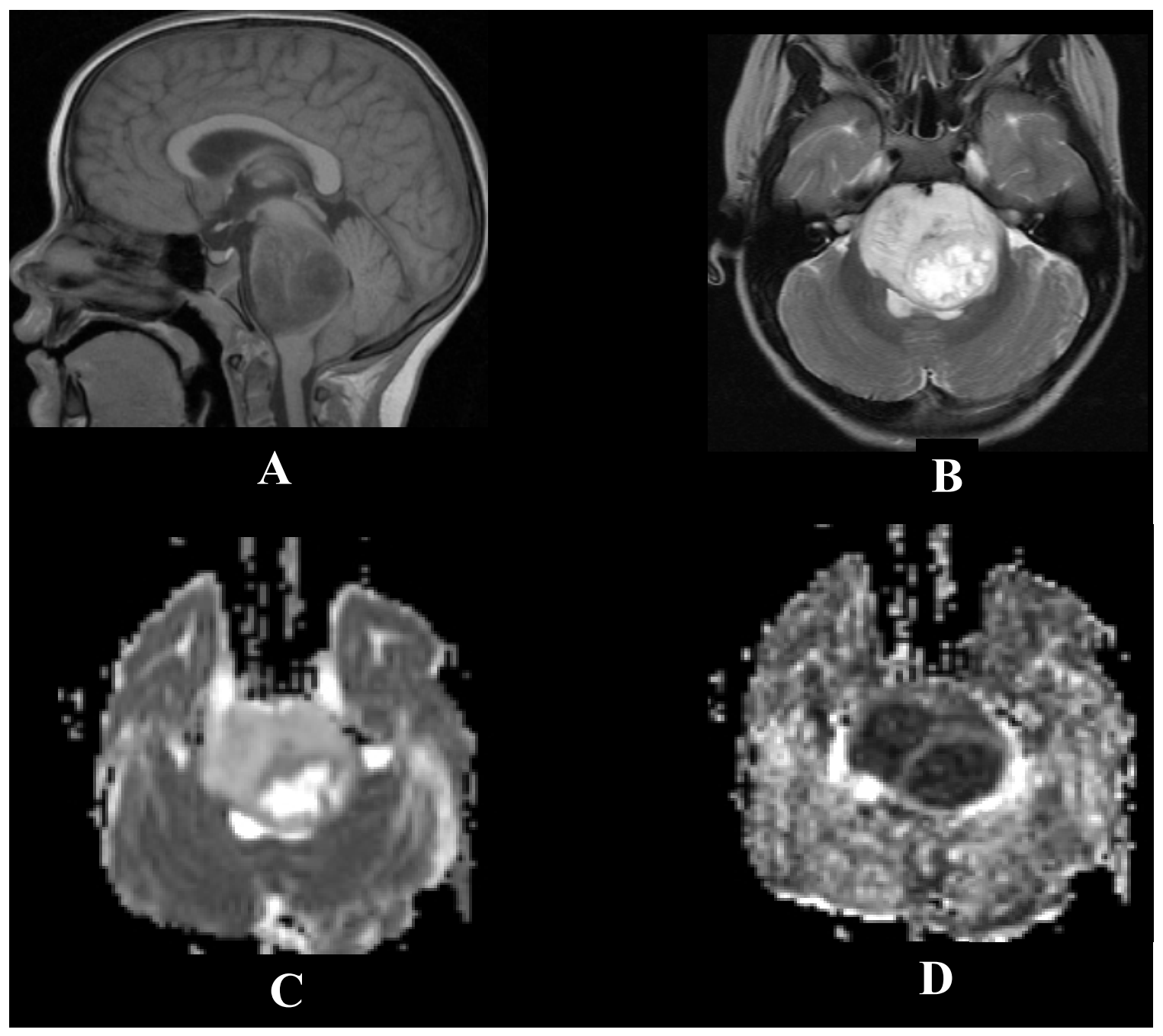

Figure 3.1 MRI and DTI images of a 15-year-old patient with diffuse brainstem glioma. A) Sagittal T1W image. The brainstem glioma is diffusely infiltrative. $B$ ) Axial T2W image. The pons appears expanded and hyperintense with an area of focal necrosis. C) Axial ADC map. The tumor demonstrates elevated diffusion, and the necrosis is extremely hyperintense. D) Axial FA map. The tumor demonstrates diminished fractional anisotropy, and necrosis is hypointense. 


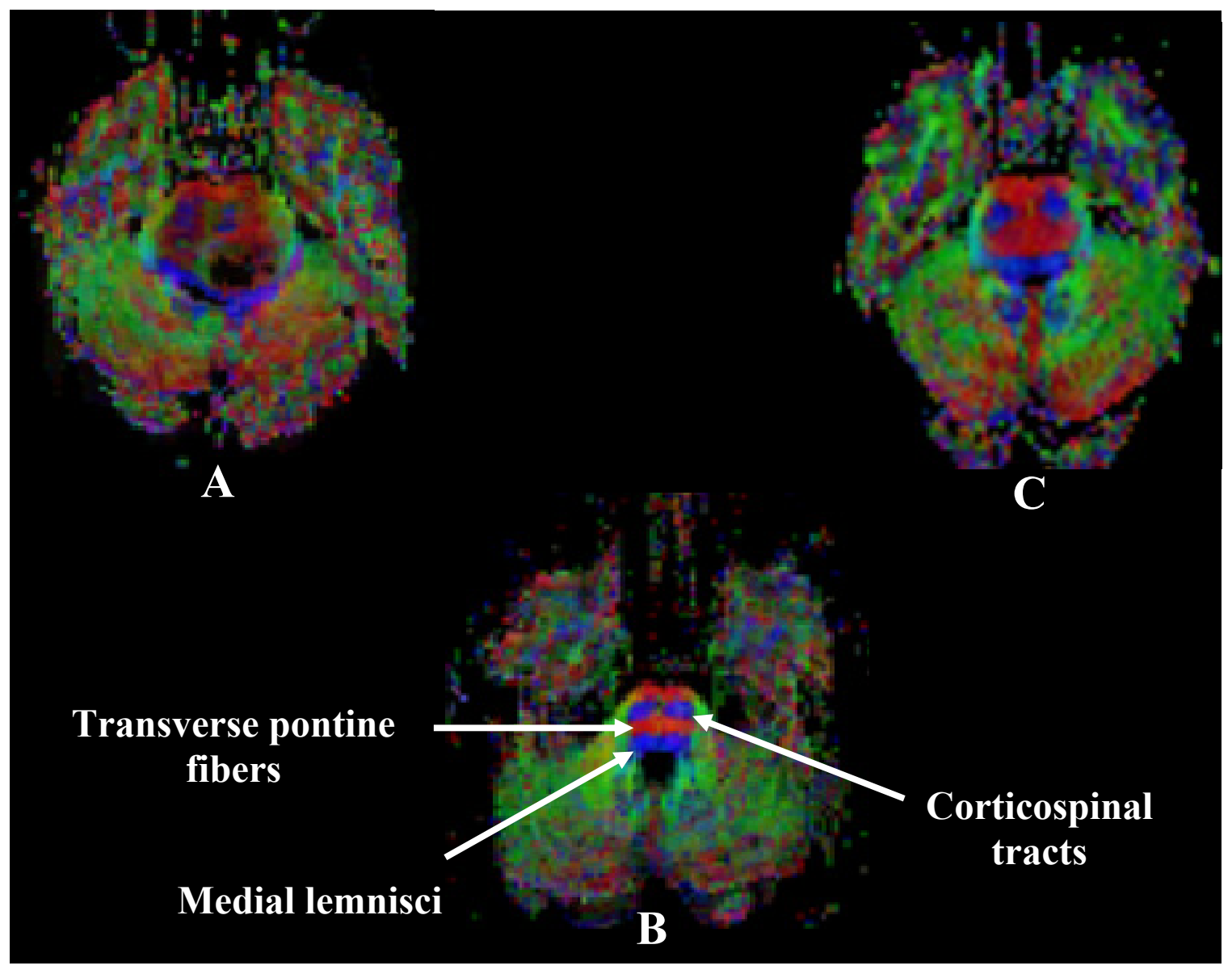

Figure 3.2 Axial diffusion tensor color maps of the brainstem at the level of the middle cerebellar peduncles. A) Patient with a brainstem glioma showing destruction of the normal anisotropy of the corticospinal tracts and posterior displacement of the medial lemnisci. B) Control image showing normal corticospinal tracts transverse pontine fibers and medial lemnisci. $C$ ) Patient with a brainstem glioma showing a diffusely infiltrating pattern. 
Both conventional MRIs (axial pre- and post-contrast T1W, T2W), and colorcoded DTI maps were analyzed for obvious tract disturbance and areas of hemorrhage or necrosis. We utilized ROIs of the white matter tracts on the color maps to obtain quantitative FA and ADC values. We also used corresponding T2W images at the level of the middle cerebellar peduncles and adapted criteria developed by Witwer et al. (2002), to classify fiber tracts into 1 of 5 categories: normal, displaced, edematous, infiltrated, or destroyed. In contrast to the Witwer study, we did not use a contralateral "normal" brain image as a control, because diffuse pontine tumors were most often bilateral. We also gathered quantitative ADC data for more in depth analysis of white matter involvement, though we did not use that information to determine tract involvement, as per Witwer's adapted criteria. Instead, we used the appearance and locations of the corticospinal, transverse pontine, and medial lemniscal tracts on the FA image and color maps (Fig 3.2).

\subsection{Classification of Tract Involvement}

Quantitative FA and ADC values from the volunteer group were used to define normal anisotropy and location of the tracts. Displaced tracts were defined as those that maintained normal anisotropy but were in abnormal locations, had an abnormal orientation on the color-coded map, or both. Edematous tracts were those that maintained normal anisotropy and orientation but demonstrated high signal intensity on T2-weighted images. Infiltrated tracts were those that demonstrated reduced aniosotropy but were still identifiable on color-coded maps. Disrupted tracts were those that had markedly reduced aniosotropy and no identifiable tracts on the color-coded map. Quantitative ADC and FA values from each ROI in the BSG group were compared with those values from the same ROI in the patient and normal control groups.

\subsection{Evaluation of Neurologic Deficits}

We retrospectively reviewed the neurologic evaluations performed on each patient with BSG before their MRI examination. Neurologic information for motor and cranial nerve findings was complete in all 7 patients, although sensory information was available for only 3 patients. DTI ROI analysis was blinded to neurologic findings. Neurologic deficits were classified into 4 categories: corticospinal, cranial nerve, sensory, and ataxia. The severity of deficit within each category was also graded as: absent if no deficit was present; mild if neurologic deficit resulted in only minimal loss of function, or in the case of cranial nerves $<50 \%$ loss of function; and moderate/severe if neurologic deficit resulted in significant loss of function, or in the case of cranial nerves $>50 \%$ loss of function. Correlation analyses were then performed to determine whether the neurologic deficit scores were associated with the quantitative DTI measurements of the corresponding anatomic structures. 


\subsection{Statistical Analysis}

The exact Kruskal-Wallis test was performed, and if a pair-wise comparison was necessary, the exact Wilcoxon-Mann-Whitney test was also employed. All tests were performed using StatXact 5 software (Cytel Software Corporation, Cambridge, MA), which was implemented using SAS version 9.1 (SAS Institute Inc, Cary, NC, USA). A $P$-value $\leq 0.05$ was considered statistically significant for the 3 -group comparisons, and a $P$-value $\leq 0.0167$ (i.e., $0.05 / 3$ ) was considered statistically significant for pair-wise comparisons. We combined data from the 2 control groups and compared the combined data with that from the BSG group. Also, we combined data sets of the 2 lower grades (absent and mild) from individual neurologic deficit categories and compared the combined data with that from the moderate/severe grade of the same deficit by using the exact Wilcoxon-Mann-Whitney test; a $P$-value $\leq 0.05$ was considered statistically significant for these comparisons.

\subsection{Conventional MRI Evaluation}

Tumors ranged in size. The axial dimensions ranged from 2.6 to $4.6 \mathrm{~cm}$ (median, $3.3 \mathrm{~cm}$; mean, $3.2 \mathrm{~cm}$ ), and the anterior-posterior dimensions ranged from 1.8 to $4.7 \mathrm{~cm}$ (median, $3.6 \mathrm{~cm}$; mean, $3.6 \mathrm{~cm}$ ). One patient's tumor had an associated focally enhanced exophytic component, and another patient's tumor had focal enhancement surrounding a region of necrosis (Table 3.1). Tumors were moderately to severely hypointense on T1W images and moderately to severely hyperintense on $\mathrm{T} 2 \mathrm{~W}$ images. The mass effect was minimal $(n=4)$, mild $(n=1)$, or moderate $(n=2)$. Only one patient had hydrocephalus at the time of imaging, but another patient had previously received a shunt. No tumor hemorrhage or metastatic disease was detected.

\subsection{DTI Evaluation}

Evaluation of the DTI color maps revealed several patterns of tumor infiltration. Overall, the tumors appeared to expand the transverse pontine components and, to a lesser extent, the corticospinal tracts (Table 3.1). In 3 patients, at least one corticospinal tract was so infiltrated that both the corticospinal and corticobulbar components were visible (Fig 3.3). The corticospinal tracts were displaced $(n=3)$ or remained in their expected locations $(n=4)$ with various degrees of expansion. In one patient, the left corticospinal tract was diminished in size and appeared slightly isotropic. In 5 patients, the sensory tracts (including the medial lemnisci and multiple cranial nerve nuclei) were posteriorly displaced, often thinned, and draped over the substance of the tumor; however, the color map of those tracts indicated that they maintained their anisotropy. 
Table 3.1 Comparison of conventional MRI and DTI measures in 7 pediatric patients with brainstem gliomas.

\begin{tabular}{|c|c|c|c|c|c|c|c|c|c|}
\hline \multirow[b]{2}{*}{$\begin{array}{c}\text { Patient } \\
\text { No. }\end{array}$} & \multicolumn{4}{|c|}{ Tumor Characteristics } & \multicolumn{5}{|c|}{ Conventional Magnetic Resonance Imaging } \\
\hline & Location & $\begin{array}{l}\text { Size } \\
(\mathrm{cm})\end{array}$ & T1W & $\mathbf{T} 2 \mathbf{W}$ & Enhancement & Focality & Necrosis & Mass Effect & Hydrocephalus \\
\hline 1 & $\begin{array}{l}\text { midbrain / } \\
\text { pons }\end{array}$ & $\begin{array}{l}4.6 x \\
3.6\end{array}$ & hypo & hyper & No & No & No & Moderate & Shunt in place \\
\hline 2 & pons & $\begin{array}{l}3.0 \mathrm{x} \\
3.4\end{array}$ & hypo & hyper & No & No & No & Minimal & No \\
\hline 3 & pons & $\begin{array}{l}2.8 \times \\
3.1\end{array}$ & hypo & hyper & No & No & No & Minimal & No \\
\hline 4 & pons & $\begin{array}{l}3.2 \mathrm{x} \\
4.3\end{array}$ & $\begin{array}{l}\text { sev } \\
\text { hypo }\end{array}$ & $\begin{array}{l}\text { sev } \\
\text { hyper }\end{array}$ & No & No & No & Minimal & No \\
\hline 5 & pons & $\begin{array}{l}3.8 x \\
4.5\end{array}$ & $\begin{array}{l}\text { sev } \\
\text { hypo }\end{array}$ & $\begin{array}{l}\text { sev } \\
\text { hyper }\end{array}$ & $\begin{array}{l}\text { Surronding } \\
\text { necrosis }\end{array}$ & No & $\mathrm{Yes}^{\mathrm{a}}$ & Moderate & Mild \\
\hline 6 & pons & $\begin{array}{l}3.9 x \\
4.7\end{array}$ & $\begin{array}{l}\text { sev } \\
\text { hypo }\end{array}$ & $\begin{array}{l}\text { sev } \\
\text { hyper }\end{array}$ & No & No & No & Mild & No \\
\hline $7^{\mathrm{b}}$ & pons & $\begin{array}{l}1.6 \mathrm{x} \\
1.0 \\
1.0 \mathrm{x} \\
0.2\end{array}$ & hypo & hyper & Minimal focal & Yes & No & Minimal & No \\
\hline
\end{tabular}


Table 3.1 (Continued).

\section{Diffusion Tensor Imaging}

\begin{tabular}{|c|c|c|c|}
\hline $\begin{array}{l}\text { Patient } \\
\text { No. }\end{array}$ & Corticospinal Tracts & Transvers Pontine Tracts & Sensory Tracts \\
\hline 1 & Bilateral severe exp, distinct CB & Severe exp of left tract & Posterior displ, noninfiltrated \\
\hline 2 & $\begin{array}{l}\text { Mild exp, lateral displ of right tract, Mild } \\
\text { reduction, medical deviation, and slightly } \\
\text { isotropic left tract }\end{array}$ & Severe bilateral exp & Posterior displ, noninfiltrated \\
\hline 3 & $\begin{array}{l}\text { Bilateral severe exp, minimal posterior displ } \\
\text { of right tract }\end{array}$ & Mild bilateral exp & Normal \\
\hline 4 & Bilateral severe exp & Severe bilateral exp & $\begin{array}{l}\text { Bilateral grossly posterior displ } \\
(\mathrm{L}>\mathrm{R})\end{array}$ \\
\hline 5 & Bilateral severe exp, distinct CB & Severe bilateral exp, necrosis & $\begin{array}{l}\text { Bilateral grossly posterior displ } \\
(\mathrm{L}>\mathrm{R})\end{array}$ \\
\hline 6 & $\begin{array}{l}\text { Bilateral severe exp, posterior displ, distinct } \\
\text { right } \mathrm{CB}\end{array}$ & $\begin{array}{l}\text { Severe, pominently anterior } \\
\text { exp }\end{array}$ & $\begin{array}{l}\text { Bilateral grossly posterior displ } \\
(\mathrm{L}>\mathrm{R})\end{array}$ \\
\hline 7 & Mild exp of left tract & Mild exp & Normal \\
\hline
\end{tabular}

a The necrotic region measured $2.2 \times 2.5 \mathrm{~cm}$.

${ }^{\mathrm{b}}$ Patient 7's tumor had an exophytic component that measured $1.0 \times 0.8 \mathrm{~cm}$.

Abbreviations: hypo, hypointense; hyper, hyperintense; sev, severe; exp, expansion; CB, corticobulbar tracts; displ, displacement; deviat, deviation. 


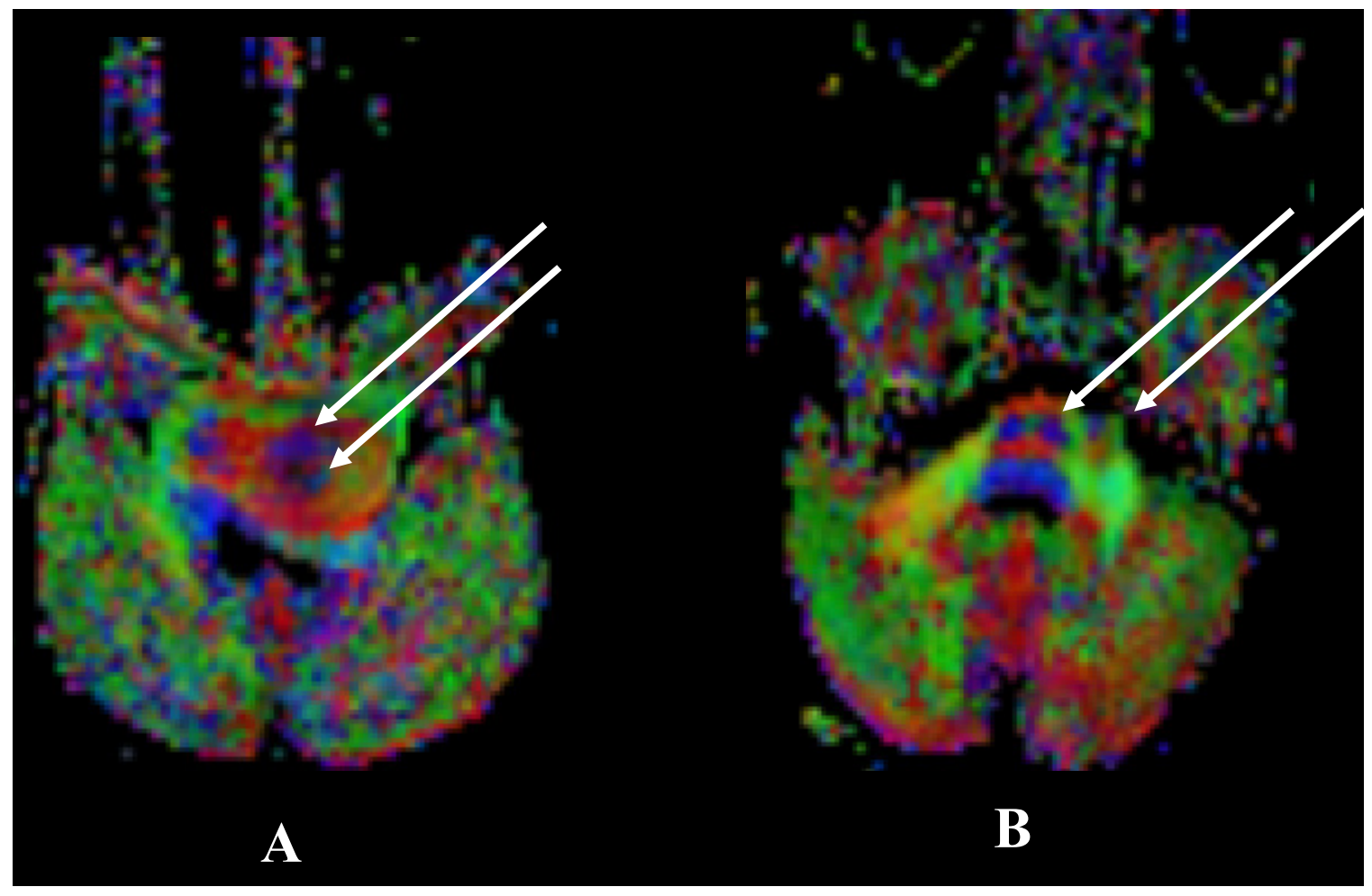

Figure 3.3 Axial diffusion tensor color maps demonstrating tract invasion.

A) 6 year-old patient with a diffusely infiltrating pontine glioma. The left corticospinal tract is enlarged, compared with the right, and tumor infiltration separates the corticospinal (anterior arrow) and corticobulbar (posterior arrow) components. B) 8 yearold patient with a focally exophytic pontine tumor. Mild lateral expansion of left corticospinal tract (medial arrow), with focally exophytic tumor (lateral arrow).

\subsection{White Matter Tract Involvement}

In accordance with the criteria listed in the Methods, we classified the status of the white matter tracks in each ROI by using their appearance on T2W images, the color maps, and FA values. The corticospinal tracts and transverse pontine fibers were more often infiltrated or destroyed than were the medial lemnisci (Table 3.2).

Median FA and ADC values of the BSG cohort at the 6 ROIs were compared with those in both control groups. The 3-way comparisons revealed that FA values of all 6 tracts were significantly decreased, and ADC values were significantly increased in patients with BSGs (Fig 3.4). In the pair-wise comparisons, the FA values of the BSG group significantly differed from those in the patient controls in the following ROIs: bilateral corticospinal tract, bilateral transverse pontine, and left medial lemniscus (Fig 3.4 A). A marginal difference was observed in the ADC values of the left corticospinal and bilateral transverse pontine tracts (Fig 3.4 B) and in the FA value of the right medial lemniscus. Interestingly, despite the differences in age and prior therapies in the patient control group, there was no significant difference in any of the ROIs between the 
Table 3.2 Color map assessment of diffusion tensor images of brainstem white matter in pediatric patients with brainstem gliomas.

\begin{tabular}{|c|c|c|c|c|c|c|}
\hline \multirow{2}{*}{$\begin{array}{l}\text { Patient } \\
\text { No. }\end{array}$} & \multicolumn{2}{|c|}{$\overline{\text { Corticospinal Tract }}$} & \multicolumn{2}{|c|}{ Transverse Pontine Tracts } & \multicolumn{2}{|c|}{ Medial Lemnisci } \\
\hline & Left & Right & Left & Right & Left & Right \\
\hline 1 & $\mathrm{D} / \mathrm{I}$ & $\mathrm{I}$ & $\mathrm{I}$ & $\mathrm{I}$ & $\mathrm{D}$ & $\mathrm{D}$ \\
\hline 2 & $\mathrm{I}$ & $\mathrm{X} / \mathrm{I}$ & $\mathrm{I}$ & $\mathrm{I}$ & $\mathrm{D}$ & $\mathrm{D}$ \\
\hline 3 & I & I & $\mathrm{I}$ & $\mathrm{I}$ & $\mathrm{D} / \mathrm{I}$ & $\mathrm{D} / \mathrm{I}$ \\
\hline 4 & I & I & $\mathrm{X} / \mathrm{I}$ & $\mathrm{X} / \mathrm{I}$ & $\mathrm{D}$ & $\mathrm{D}$ \\
\hline 5 & $\mathrm{X} / \mathrm{I}$ & $\mathrm{X} / \mathrm{I}$ & $\mathrm{X} / \mathrm{I}$ & $\mathrm{X} / \mathrm{I}$ & I & I \\
\hline 6 & I & $\mathrm{X} / \mathrm{I}$ & I & I & $\mathrm{N}$ & I \\
\hline 7 & I & $\mathrm{N}$ & $\mathrm{N}$ & $\mathrm{N}$ & $\mathrm{N}$ & $\mathrm{N}$ \\
\hline
\end{tabular}

The condition of the tracts was classified as; displaced (D), edematous (E), infiltrated (I), normal $(\mathrm{N})$, or destroyed $(\mathrm{X})$.
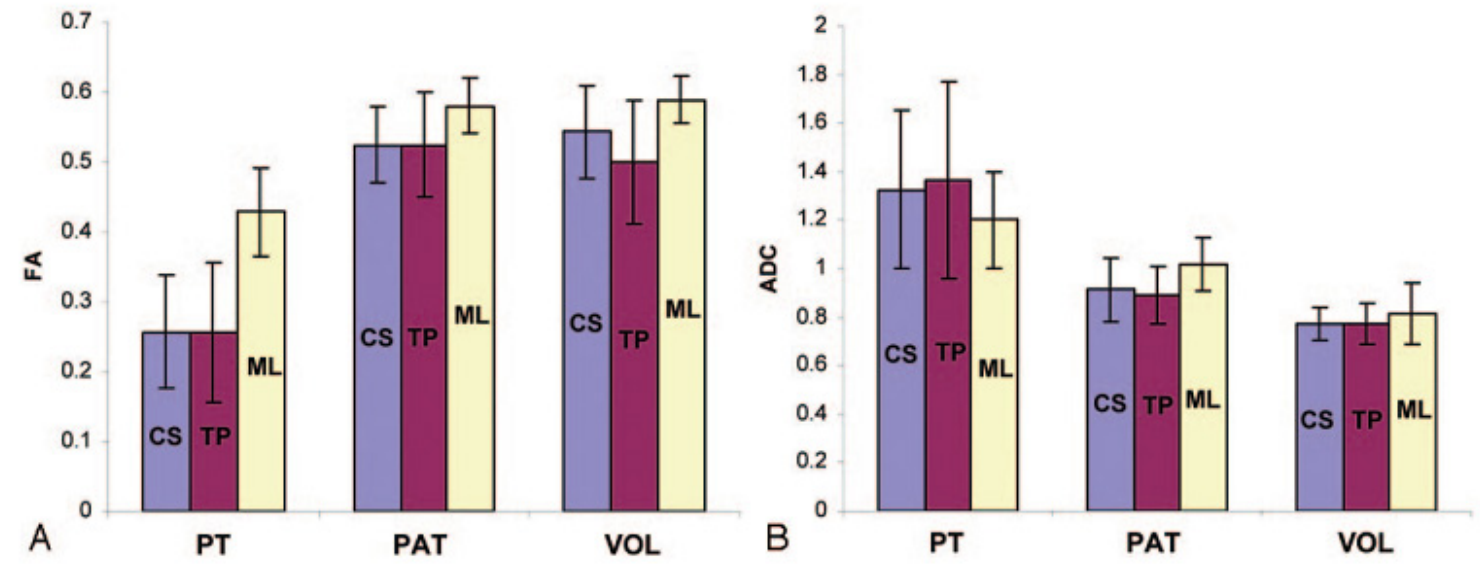

Figure 3.4 Diffusion tensor imaging parameters for major white matter tracts in the brainstem. A) Fractional anisotropy (FA). B) Apparent diffusion coefficient (ADC). Data are shown for the corticospinal (CS, blue bars), transverse pontine (TP, red bars), and medial lemnisci (ML, yellow bars). Subject groups included patients with brainstem glioma (PT), patient control (PAT), and healthy volunteer (VOL) groups. 
patient control and volunteer groups; only a marginal difference was observed in the $\mathrm{ADC}$ value of the right medial lemniscus. Consequently, a pair-wise comparison of data from the BSG cohort and the combined control groups demonstrated significant differences in FA and ADC measures in all 6 ROIs.

Although we attempted to determine whether there was a correlation between the severity of neurologic deficits in the patients with BSGs and the median FA and ADC values of their corticospinal, transverse pontine, and sensory tracts, this analysis was limited by small sample size, because the neurologic examination data were gathered retrospectively and sensory data was incomplete. In spite of these limitations, the comparison of severity of cranial nerve deficits with the average FA value of the transverse pontine tract showed a marginal difference $(p=0.057)$, which could be clinically significant. We compared data from patients with normal or mild cranial nerve deficits with that from patients with moderate/severe cranial nerve deficits. We performed a similar comparison using data from patients with normal or mild ataxia and those with severe ataxia. There was no significant difference in any comparison.

Various combinations of the following symptoms were experienced by the 7 patients with BSGs at time of imaging: long tract signs $(n=3)$, cranial nerve deficits ( $n=$ 5 ), and ataxia $(n=4)$ (Table 3.3). Sensory involvement was noted as "normal" in 3 patients and not recorded in the evaluations of the other four.

Table 3.3 Neurological deficits in 7 pediatric patients with brainstem gliomas.

\begin{tabular}{|c|c|c|c|c|}
\hline \multirow{2}{*}{$\begin{array}{l}\text { Patient } \\
\text { Number }\end{array}$} & \multicolumn{4}{|c|}{ Neurological Deficit (grade and location) } \\
\hline & Corticospinal & Cranial Nerves & Sensory & Ataxia \\
\hline 1 & Mild (left) & Mild (left) VI & n.a & Absent \\
\hline 2 & Mild (right) & M/S (left) VI,VII,IX & n.a & Absent \\
\hline 3 & Absent & Absent & n.a & Absent \\
\hline 4 & Absent & $\mathrm{M} / \mathrm{S}$ (bilat) VI, (left) VII, IX & Absent & $\begin{array}{l}\mathrm{M} / \mathrm{S} \text { (bilat), } \\
\text { worse on left }\end{array}$ \\
\hline 5 & Absent & $\begin{array}{l}\text { M/S Left gaze, VI, VII, and } \\
\text { partial IX }\end{array}$ & Absent & $\begin{array}{l}\text { Severe gait, } \\
\text { moderate } \\
\text { bilateral limb } \\
\text { dysmetria }\end{array}$ \\
\hline 6 & $\begin{array}{l}\text { Mild (bilat) } \\
\text { worse on left }\end{array}$ & $\begin{array}{l}\mathrm{M} / \mathrm{S} \text { (bilat) VI, IX, worse on } \\
\text { right }\end{array}$ & n.a & $\begin{array}{l}\text { Mild gait, left } \\
\text { limb } \\
\text { dysmetria }\end{array}$ \\
\hline 7 & Absent & Absent & Absent & $\begin{array}{l}\text { Mild limb } \\
\text { dysmetria }\end{array}$ \\
\hline
\end{tabular}

Deficits were classified into 2 grades: absent/mild and moderate/severe (M/S), n.a., not available. 


\subsection{Discussion}

We describe quantitative and significantly altered DTI measures (FA and ADC) in the brainstem tracts of 7 patients with BSGs $(P<0.05)$, compared with those values in the two control groups. BSGs comprise approximately $15 \%$ of pediatric brain tumors, and the most common subtype of BSG is diffuse pontine glioma [43]. Most children with apparent diffuse pontine gliomas die of disease within 18 months of diagnosis, despite contemporary radiation therapy and chemotherapy [43-45]. The current principal diagnostic modality for BSGs is MRI, and there are several key grading systems that use MRI findings to classify brainstem tumors by location and focality [31,44,46,47]. There is still debate in the literature about whether the presence of enhancement is a useful prognostic sign $[48,49]$. However, there is no question that patients with focal tumors, as seen on conventional $\mathrm{T} 2 \mathrm{~W}$ images, benefit from information obtained from the stereotactic biopsy.

A certain percentage of patients with focal BSGs have lower-grade histology, hence they also have different treatment options and prognoses [47]. Lesniak et al (2003) found that of the 57 patients with enhancing brainstem tumors who were surgical candidates, $60 \%$ had low-grade histology (30 juvenile pilocytic astrocytoma, 3 ganglioglioma, 1 oligoglioma), and 40\% had higher-grade histology (12 glioblastoma multiforme, 10 fibrillary astrocytoma and 1 primative neuroectodermal tumor) [49]. Both location and focality as defined by MRI have substantial prognostic significance. The probability of 5-year overall survival of patients with tumors of the midbrain ranged from 72 to $100 \%$; that of patients with tumors of the pons was $18 \%$; that of patients with tumors of the medulla ranged from 50 to $64 \%$; and that of patients with focal tumors ranged from 56 to $100 \%$. The 5 -year overall survival of patients with diffuse brainstem tumors ranged from 18 to $20 \%$ [48]. Six of our 7 patients had classic diffuse BSGs, as determined by imaging; one patient had a focally invasive tumor with an enhancing exophytic component.

The general imaging features of diffuse and focal BSGs have been well described in the literature $[31,48]$. We employed quantitative DTI to explore the relations between the involvement of the corticospinal, transverse pontine, and medial lemnisci brainstem tracts in 7 patients with BSGs with various degrees of invasion. Our analysis revealed both qualitative differences in the DTI color maps and statistically significant differences in the FA and ADC values of these tracts when compared with those of two control groups. Therefore, DTI shows promise in furthering our understanding of the effects of brainstem tumors on nearby white matter tracts.

Stieltjes et al. (2001) recently used the brainstem as a model to evaluate the ability of quantitative DTI to assess densely packed, well-known fiber tracts in 6 healthy adults [50]. They identified the corticospinal tracts; the superior, medial, and inferior cerebellar peduncles; and the medial lemnisci and evaluated their FA and ADC values. In our study, not only were these white matter tracts clearly seen in patients with BSGs, but also substantial differences in the FA and ADC values were found in a comparison of patient and the control groups. Certainly the mean corticospinal tracts and medial 
lemnisci FA values and standard deviations for our patient control and volunteer groups compared favorably with data published by Stieltjes et al. [50].

Sinha et al. explored the regional mean diffusivity (ADC) values of high-grade gliomas in 9 adults [35]. They found clear quantitative differences in ADC values of different tissues; the highest ADC was observed in the necrotic tumor core, followed by edematous brain, enhancing tumor core, enhancing tumor margin, and normal brain. A recent study comparing quantitative $\mathrm{ADC}$ values between three basic histopathologic pediatric tumor types (low-grade gliomas, embryonal tumors, and nonembryonal highgrade tumors) in 12 patients demonstrated that ADC is inversely correlated with tumor cellularity [32]. This finding contributes another diagnostic aspect of tumor characterization. Although we did not examine the boundaries of the diffuse tumors, our finding that the tracts of the patients with BSGs had elevated ADC values and were hyperintense on $\mathrm{T} 2 \mathrm{~W}$ images supports earlier findings of relatively low cellularity of these infiltrating tumors [30]. Recent advances in DTI have permitted the evaluation of white matter tract anisotropy and the calculation of the principal eigenvectors of known white matter tracts [42,51]. Pajevic and Pierpaoli developed methods to create directionally coded color maps of DTI data in which the intensity of the colors corresponds to the magnitude of the anisotropy in the white matter tracts [42,51]. These and other advances were invaluable in taking DTI color mapping to the clinical arena $[52,53]$.

Witwer et al. recently described preoperative DTI color mapping to augment surgical planning in 9 adult patients with supratentorial tumors [36]. In that study, the relations were examined between the primary tumor and characteristics of the nearby white matter tracts (i.e., location, orientation, edema, infiltration, displacement, and disruption), as compared with the contralateral normal hemisphere. Results from this study substantially improved preoperative planning, and they suggested that intact white matter tracts, including those displaced by the tumor but having normal FA values, are present in regions of the brain that appear abnormal on conventional MR images.

In our study, the patterns of diffuse tumor involvement in 6 patients with BSGs were similar by conventional imaging, but their patterns of tract involvement were quite varied by DTI. As one would expect, the color map showed evidence of diffuse infiltration of the corticospinal and transverse pontine tracts. We noted bilateral enlargement of the corticospinal and transverse pontine tracts in many patients but frequent, apparently random displacement of the individual corticospinal tracts and posterior displacement of the medial lemnisci. Patient 7 had a more focal lesion, which enlarged the left corticospinal tract, and an enhancing exophytic component that had been stable for years. We also observed that the corticospinal tracts and transverse pontine fibers were more often infiltrated or destroyed than were the medial lemnisci. This finding supports the common clinical triad of presenting symptoms in children with BSG, i.e., long tract signs, ataxia, and cranial nerve deficits [45]. Clinical features and conventional MRI are important elements of diagnosis and treatment planning for patients with BSGs $[31,48,49]$, and our findings indicate that DTI evaluation may also contribute to clinical management of such cases. A large prospective study would be 
necessary to demonstrate a correlation between decreased FA values and severity of neurologic deficits and to determine if there is a threshold below which decreased FA values become clinically apparent.

Although stereotactic biopsy of suspected focal tumors has acceptable risks, attempted partial resections of low-grade tumors for tumor debulking and decompression can result in serious morbidites. Lesniack et al. argued that in spite of the obvious benefits of careful resection of benign lesions, surgery was not without serious risks [49]. While most of the patients experienced improvement in neurologic deficits after their surgical procedure, significant morbidity was described (i.e., 17.5\% experienced moderate disability, $3.5 \%$ severe disability, and $1.7 \%$ vegetative state). We found that DTI (including color maps) provides a wealth of information that until now has not been available in surgical planning for patients with BSGs. It remains to be seen whether DTI can further our understanding of tract displacement and infiltration and facilitate surgical planning in patients with known focal lesions.

Important limitations of this retrospective study were discrepancy in age between the patient and control groups, and limited sensory information in the neurologic evaluations in the patient group. Although an age-matched control group is generally the ideal in clinical studies, the median age of our patient group was 7 years. This is well above 2 years, the age of relative stability of FA and ADC values in central white matter pathways [13]; therefore it is reasonable to assume that the differences in ages between the patient group and control groups in this study did not contribute to differences in the data. Though this study was limited by the small number of subjects, we found a potential clinically significant association between cranial nerve deficit severity and decreased FA.

\subsection{Conclusion}

This study was the first to evaluate the quantitative DTI measures of FA and ADC in pediatric patients with diffuse and focal BSGs and in those with "normal" brainstem, as determined by conventional MRIs. Our results indicate that DTI analysis can delineate tract invasion and displacement. These tools may help to better discriminate between diffuse and focal brainstem tumors in the future, and may be useful for guiding surgical biopsies. DTI analysis also shows promise of providing quantitative measures of risk stratification, prognosis, and treatment response. We conclude that the results from this retrospective study support the expansion of this research in a large, prospective study in pediatric patients with BSGs. 


\section{Chapter 4. Axonal Degeneration in Pediatric Brain Tumor Patients}

\subsection{Introduction}

Diffusion tensor imaging and tractography are powerful tools to evaluate the integrity of white matter tracts in a variety of central nervous system disease processes. Although DT imaging can detect early axonal degeneration of pyramidal tracts after stroke [54-57], its utility in brain tumors has not been definitively reported [58,59]. Wallerian degeneration (WD) describes the antegrade degeneration of axons and their surrounding myelin sheaths after injury or death of the associated proximal axons or cell bodies [60,61]. In the brain, conventional MR imaging has been used to characterize WD corresponding to axonal and myelin breakdown $[62,63]$. More recently, DT imaging and tractography have been used to quantify the degree of clinical and subclinical injury to white matter tracts [64-66].

It has also been suggested that reductions in anisotropy can correlate with a loss of tract integrity, and DT imaging has shown promise in predicting postoperative motor function following supratentorial tumor surgery [67]. Recent quantitative advances in DT imaging have helped explain the relationship of brainstem tumors to motor and sensory tracts, and DT imaging now provides a sensitive measure of tract invasion [68]. Diffusion tensor imaging also yields additional information for the surgical planning of partially exophytic cervicomedullary brainstem tumors (Phillips, et al. 2005). Further characterization of brainstem tumors by DT imaging may help differentiate focal from diffuse tumors. We explored the value of performing DT imaging and tractography in children with brainstem tumors to demonstrate degeneration of motor and sensory tracts remote from the tumor. We hypothesized that FA would be lower and apparent diffusion coefficient would be higher than those measures obtained in the same regions on MR images obtained in healthy volunteers. Confirmation of this hypothesis would provide evidence of axonal degeneration in pediatric patients with brainstem tumors, with possible implications for surgical planning.

\subsection{Methods}

We performed a cross-sectional analysis of FA, ADC, and tractography results of sensory and motor tracts in 7 pediatric patients harboring brainstem tumors (median age 7 years, range $2-18$ years). The data were retrospectively compared with those obtained earlier in 8 healthy adult volunteers whose ages ranged from 21 to 37 years. At the time of DT imaging, 3 patients had received no tumor-related treatment. One patient had undergone chemotherapy alone. Three patients had undergone biopsy or debulking surgery, and 2 of these had also received posterior fossa irradiation and chemotherapy. Neurological examinations within 30 days of imaging revealed abnormalities of gait (5 patients), cranial nerves ( 3 patients), dysmetria ( 3 patients), spasticity ( 1 patient), and hemiparesis (1 patient). Tumors were characterized by imaging as classic if they were diffusely infiltrating, expansive, occurring in a predominantly pontine location, and as 
other if they were located predominantly outside the pons or had a dominant exophytic component (Table 4.1). All patients harboring tumor had residual disease present at the time of evaluation.

\subsection{Conventional Imaging Studies}

All examinations were performed with a Siemens Symphony 1.5-T imager using a quadrature head coil. Conventional MR images, including T2-weighted sequences, FLAIR images, and pre- and postcontrasted T1-weighted images, were obtained in the patient group for diagnostic purposes.

\subsection{Diffusion Tensor Imaging}

Diffusion weighted, echo planar images were acquired with a double spin echo pulse sequence. Diffusion tensor images were calculated using the Statistical Parametric Mapping DT imaging toolbox (Poldrack; http://sourceforge.net/projects/spm-toolbox/) developed for SPM99. Our threshold for inclusion in white matter tracts was an FA $>0.2$, which corresponds to the default for the Massachusetts General Hospital tractography software. The FA and ADC maps were derived from the full DT at each voxel. To aid in the visualization of fiber tracts, we used red-green-blue orientation color maps to demonstrate the magnitude and direction of fiber tracts (Fig. 4.1) [42]. For descriptive purposes, white matter tracts were classified as follows, based on their appearance on the color maps adapted from Witwer et al: 1) Normal tracts had normal size, location, and orientation with normal FA values. 2) Infiltrated tracts were of various sizes but were normally located and had reduced FA values. 3) Reduced tracts were significantly smaller than those of normal volunteers. 4) Expanded tracts were significantly larger than those of normal volunteers. 5) Displaced tracts were altered in their location but had normal FA values. 6) Destroyed tracts retained no normal signal on the color map, and FA values were close to 0 .

\subsection{Regions of Interest Analysis}

Base DT images were masked to exclude regions of the infratentorium. This reduced misalignment errors due to artifacts from post surgical debris. These images were then realigned to correct for interimage head motion and imager drift, and normalized to the Montreal Neurological Institute brain template using the standard SPM 99 image processing toolbox $[69,70]$.

Coordinates from the normalized images were converted from Montreal Neurological Institute space to Talairach space using the transformation method developed by the Medical Research Council Cognition and Brain Sciences Unit [70,71]. (The transformation is available online at http://www.brainmap.org/icbm2tal/index.html). The probable locations of white matter tracts were identified on FA maps in the axial, 
Table 4.1 Imaging characteristics of brainstem tumors.

\begin{tabular}{|c|c|c|c|c|c|c|c|}
\hline \multirow{2}{*}{\multicolumn{2}{|c|}{$\begin{array}{l}\text { Case Predominant Tumor } \\
\text { No. Location }\end{array}$}} & \multirow{2}{*}{$\begin{array}{l}\text { Imaging } \\
\text { Type }\end{array}$} & \multirow{2}{*}{$\begin{array}{l}\text { Tumor } \\
\text { Size }\end{array}$} & \multicolumn{2}{|c|}{ Signal Intensity - Signal Tensity - } & \multirow[t]{2}{*}{ Enhancement } & \multirow[t]{2}{*}{ Focality } \\
\hline & & & & T1 & $\mathbf{T 2}$ & & \\
\hline 1 & $\mathrm{rt}>$ lt pontomedullary & classic & $5 \times 3.7$ & hypo & hyper & $\begin{array}{l}\text { focally at site of } \\
\text { bleed }\end{array}$ & diffuse \\
\hline 2 & lt $>$ rt pontomedullary & classic & $3 \times 2.1$ & hypo & hyper & none & diffuse \\
\hline 3 & $\begin{array}{l}\text { lt }>\text { rt pons, middle } \\
\text { cerebral peduncle }\end{array}$ & classic & $2.3 \times 2.6$ & hypo & hyper & $\begin{array}{l}\text { minimal } \\
\text { peripheral }\end{array}$ & $\begin{array}{l}\text { diffuse, slightly } \\
\text { eccentric }\end{array}$ \\
\hline \multirow[t]{2}{*}{4} & $\begin{array}{l}\text { It middle cerebullar } \\
\text { peduncle }\end{array}$ & other & $1.7 \times 1$ & hypo & slightly hyper & none & $\begin{array}{l}\text { partially } \\
\text { infiltrative }\end{array}$ \\
\hline & $\begin{array}{l}\text { lateral pons, venrtally } \\
\text { exophytic }\end{array}$ & & $0.7 \times 0.4$ & hypo & hyper & central & $\begin{array}{l}\text { partially focal, } \\
\text { well } \\
\text { marginated }\end{array}$ \\
\hline 5 & lt $>$ rt medulla & other & $2.1 \times 2.2$ & iso & hyper & none & $\begin{array}{l}\text { diffuse } \\
\text { expansion, } \\
\text { poorly } \\
\text { marginated }\end{array}$ \\
\hline 6 & $\begin{array}{l}\mathrm{rt}>\text { dorsally exophytic } \\
\text { medullary mass }\end{array}$ & other & $1.6 \times 1.5$ & hypo & hyper & brilliant & $\begin{array}{l}\text { well } \\
\text { marginated }\end{array}$ \\
\hline 7 & $\begin{array}{l}\text { rt middle cerebellar } \\
\text { peduncle/ pons }\end{array}$ & other & $0.9 \times 1.4$ & iso/hypo & iso/hyper & focal brisk & $\begin{array}{l}\text { poorly } \\
\text { marginated/ } \\
\text { infiltrative }\end{array}$ \\
\hline
\end{tabular}

* hyper $=$ hyperintense , hypo $=$ hypointense $;$ iso $=$ isointense. 


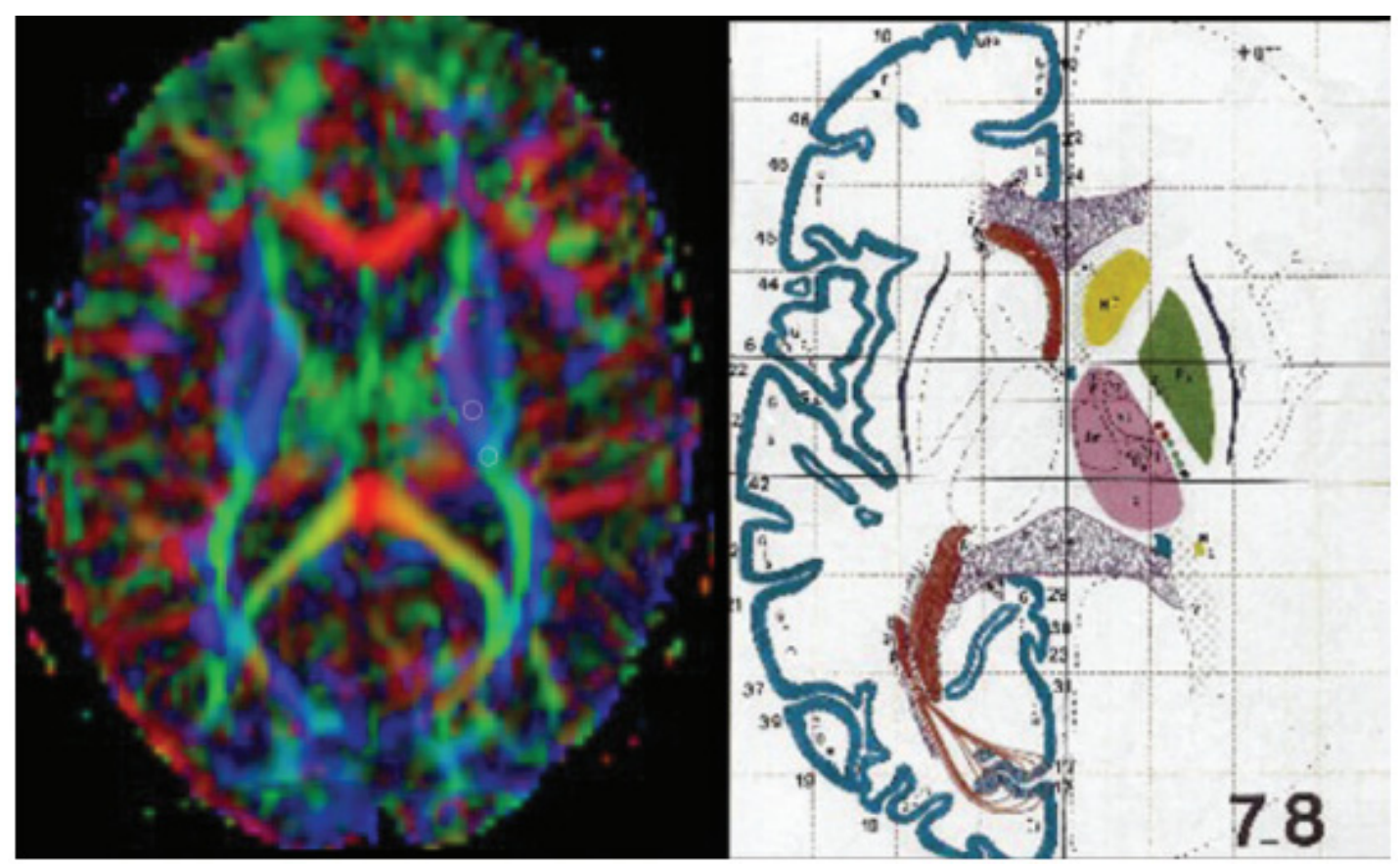

Figure 4.1 Color maps of motor and sensory ROIs. Left: A color map used to demonstrate the direction of fiber tracts. Note the anterior position of the motor and posterior position of the sensory ROIs in the posterior limb of the internal capsule (circles). Right: Precise locations of white matter tracts were identified using Talairach's atlas. Fractional anisotropy, ADC, and eigenvector maps were calculated. Reprinted by permission of Talairach J, Tournoux P: Co-Planar Stereotaxic Atlas of the Human Brain. New York: Thieme Publishers, 1988. 
sagittal, and coronal planes. The locations of the ROIs were iteratively improved by checking the appearance with the determined 3D coordinates fiber tract visualization with red green-blue color maps, FA maps, and ADC maps (Fig 4.1). Additionally, Talairach Deamon information software (Talairach Daemon Client [Version 1.1], Research Imaging Center, University of Texas Health Science Center) and the Thalamic Connectivity Atlas developed by members of the Centre for Functional MRI of the Brain (University of Oxford) were used to confirm coordinate locations in ventral posterolateral thalamic nuclei and supratentorial sensory and motor white matter [72]. The mapping process ensured selection of ROIs with high probability of appropriate sensory and motor connections. The ROIs (average size 16 pixels) were selected in bilateral sensory tracts (referred to as medial lemnisci in the brainstem, and sensory tracts above the tentorium) and included the pons, midbrain, ventral posterolateral thalamic nuclei, internal capsule, and subcortical white matter. The bilateral motor ROIs (that is, corticospinal tracts) were selected at the level of the pons, midbrain, internal capsule, corona radiata, and subcortical white matter. The ROIs could not be placed in the medulla due to artifact from the skull base. They were selected on color maps and then copied to FA and ADC maps. The FA and ADC values were measured at each ROI for a total of 328 ROIs and 656 patient and normal volunteer data points. For visualization, tractographic images were generated using DTI Task Card software (Version 1.69, Massachusetts General Hospital NMR Center) to compare sensory and motor tracts at multiple levels in patients with those in healthy volunteers (Fig 4.2). Additional ROIs (average size 16 pixels) were obtained at the level of the splenium and genu of the corpus collusum (patients and normal volunteers, 14 ROIs and 28 data points each).

\subsection{Statistical Analysis}

The one-sided exact Wilcoxon-Mann-Whitney test was used to assess whether the FA and ADC values at each of the 10 ROIs in the combined patient group was less or greater than those in the healthy volunteer group. We performed the two-sided exact Wilcoxon-Mann-Whitney test to compare FA and ADC values at the level of the splenium and genu of the corpus callosum between the 2 groups. All tests were performed using StatXact-5 software (Cytel SoftwareCorp.) implemented using SAS Version 9.1. A probability value $\mathrm{P}<0.05$ was considered statistically significant.

\subsection{Result}

\subsubsection{Conventional MR Imaging}

Tumors predominantly exhibited a hypointense signal on T1-weighted images and hyperintense signal on T2-weighted images. The tumors included 3 classic and 4 other brainstem tumors. The latter group comprised 2 middle cerebellar peduncle tumors with lateral pontine involvement and 2 medullary tumors (Fig 4.3), 1 of which included 

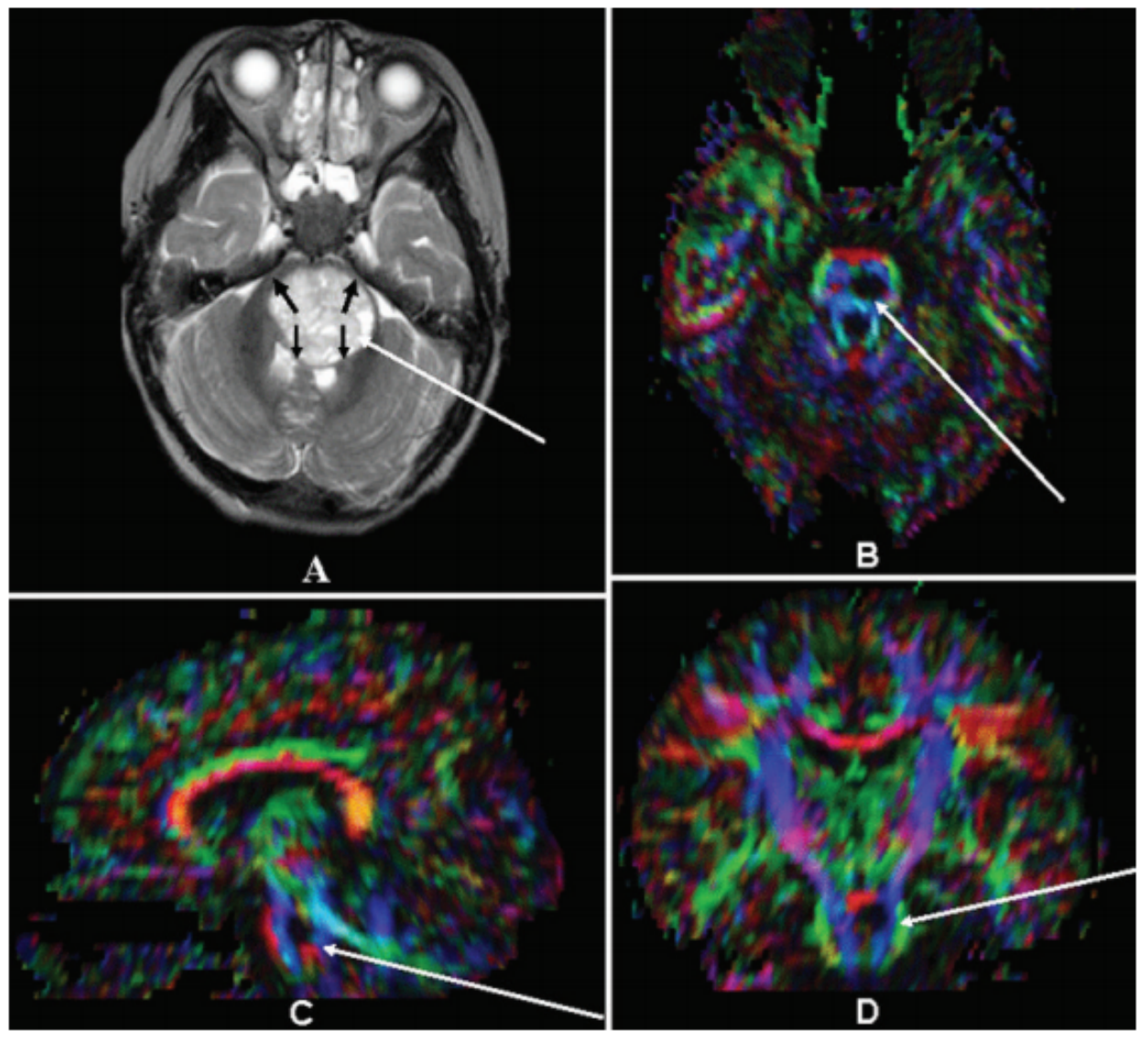

Figure 4.2 White matter images of representative focal tumor. Preoperative T2weighted MR image $(A)$ demonstrating displaced motor (thick black arrows) and sensory (thin black arrows) displaced by tumor (white arrow). Axial $(B)$, sagittal $(C)$, and coronal $(D)$ postoperative tractographic images showing surgical cavity (white arrows). 

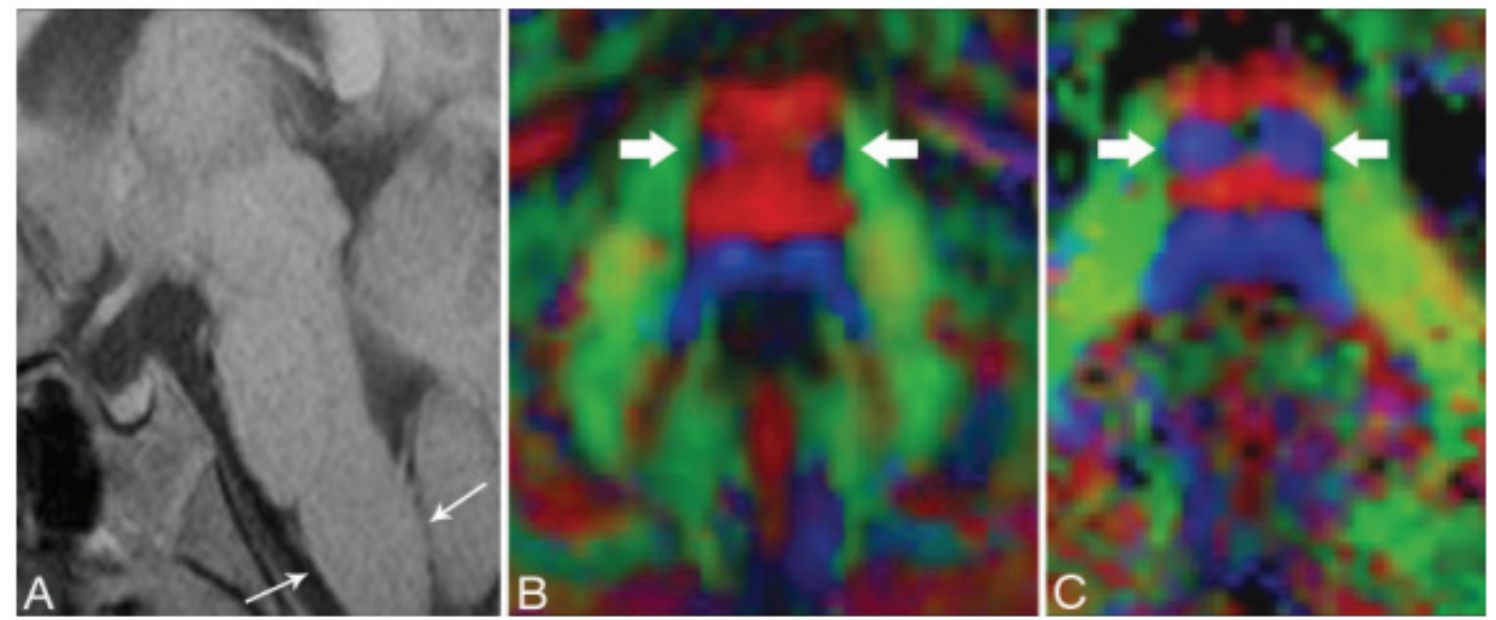

Figure 4.3 Diffusely infiltrating medullary tumor. A) Sagittal T1-weighted MR image demonstrating the diffusely expanded medulla (thin arrows) and normal sized pons. $B$ ) Color map obtained in a patient with medullary tumor at the level of the pons. Bilateral corticospinal tracts (thick arrows) can be seen. C) Color map obtained in a healthy volunteer at the level of the pons. The bilateral corticospinal tracts (thick arrows) are larger, supporting the concept of degeneration of these tracts in a patient with medullary tumor. 
prominent exophytic components. Enhancement was common in both tumor subtypes, ranging from brilliant enhancement of a dorsally exophytic medullary tumor to focal punctate enhancement medially at a site of bleeding in a diffusely infiltrating pontomedullary tumor. The mean tumor size was $2.5 \times 3 \times 2.1 \mathrm{~cm}$ (median size 2.332 .1 $\mathrm{cm}$ ) in the axial and anteroposterior directions. In spite of variable locations, 5 of the 7 tumors had predominantly diffusely infiltrating components (Table 4.1).

\subsubsection{Color Map Analysis}

Color maps at the level of the brainstem tumor were used to evaluate 14 individual sensory (medial lemnisci) tracts, and 14 motor (corticospinal) tracts. Six of 14 medial lemnisci tracts were infiltrated, and 2 were displaced posteriorly. Eleven of 14 corticos pinal tracts were infiltrated, and 1 was destroyed. Four corticospinal tracts were also displaced by the tumor mass; of those, 3 were displaced posteriorly (Fig 4.4). Only 2 corticospinal tracts were considered normal, and those were detected in the dorsally exophytic medullary tumor (Table 4.2).

\subsubsection{Quantitative DT Imaging}

The median FA values were significantly lower $(p<0.05)$ in the combined patient group than the healthy volunteer group in both sensory and motor tract ROIs (Tables 4.3 and 4.4). Significant sensory tract differences were identified from the bilateral pons to the subcortical white matter. Significant motor tracts differences were identified as bilateral pons, left midbrain, and right corona radiata. There was a bimodal pattern of antegrade decreased FA involving second-order $\left(2^{\circ}\right)$ and third-order $\left(3^{\circ}\right)$ sensory axons and a trend toward more pronounced retrograde decreased FA of motor axons closest to the tumor with gradual increases in FA upstream of the tumor. There were also scattered regions with significantly increased ADC in the sensory tracts, (right pons, left midbrain, left internal capsule, and the bilateral subcortical white matter), and motor tracts (right subcortical white matter). There was no significant difference of diffusion parameters in the genu ( $\mathrm{p}=0.69$ and $0.98, \mathrm{FA}$ and ADC, respectively) or splenium ( $\mathrm{p}=0.72$ and 0.96 , FA and ADC, respectively) of the corpus callosum between the patient and volunteer groups.

\subsection{Discussion}

We sought to determine whether DT imaging could be used to analyze the effects of tumor and/or its treatment on the integrity of nearby and distant sensory and motor white matter tracts. We found that FA and ADC values differed significantly in sensory and motor tracts between the patient and healthy volunteer groups but not in the presumably uninvolved tracts of the corpus callosum. These findings have clinical implications for planning biopsies or debulking of unusual brainstem tumors, and they can help improve prognostication of functional tract recovery following therapy. The 

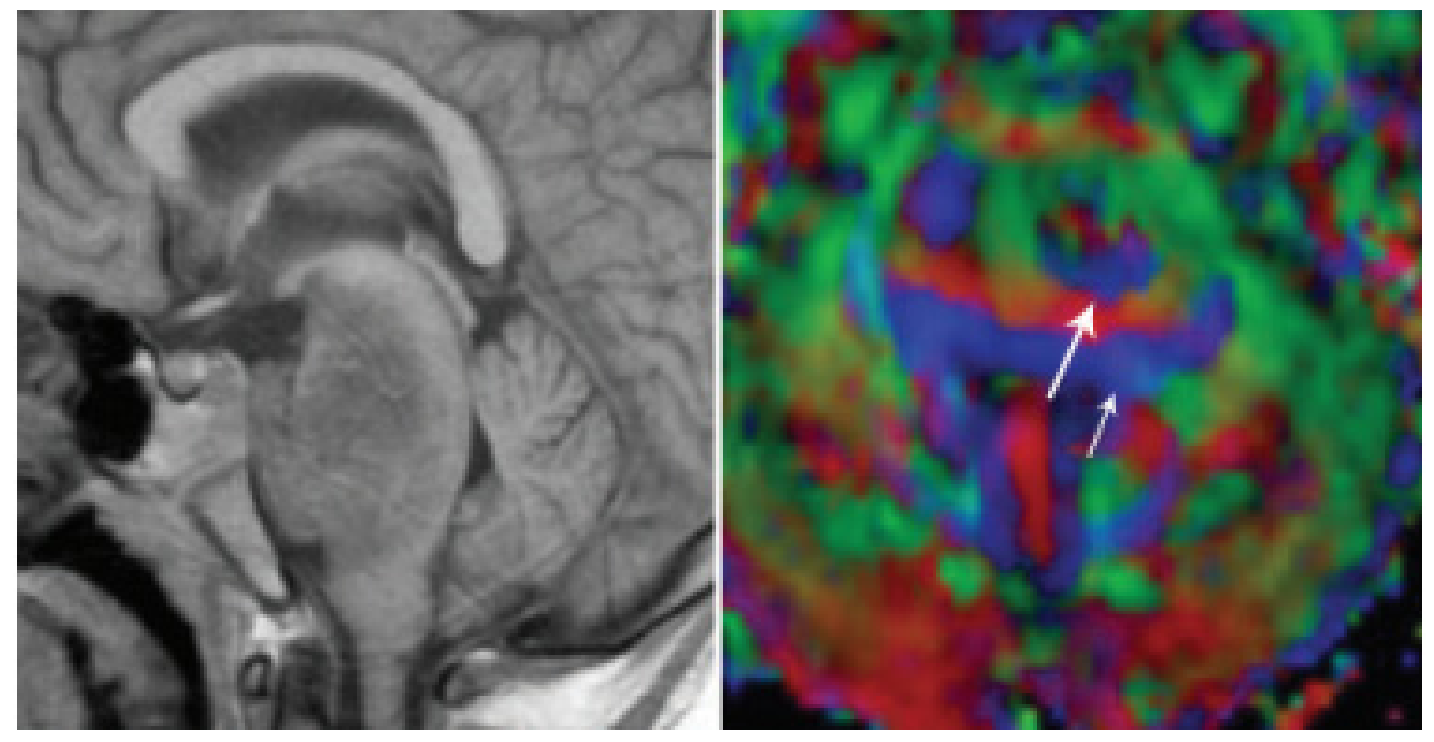

Figure 4.4 Classic pontine glioma. Left: Sagittal T1-weighted MR image revealing a diffusely infiltrative pontine tumor. Right: Axial color map demonstrating a posteriorly displaced and expanded left corticospinal tract (larger white arrow). The left sensory tract is also posteriorly displaced (smaller white arrow). 
Table 4.2 Analysis of colormap at the level of the brainstem.

\begin{tabular}{llllll} 
& \multicolumn{2}{c}{ Corticospinal Motor Tracts } & & \multicolumn{2}{c}{ Medial Lemniscal Sensory Tracts } \\
\cline { 2 - 3 } \cline { 5 - 6 } Case No. & Left & Right & Left & Right \\
\hline 1 & infiltrated, reduced size & infiltrated, reduced size & & normal & normal \\
2 & infiltrated & infiltrated & normal & normal \\
3 & infiltrated, posteriorly & infiltrated, anteriorly & & normal & mildly infiltrated \\
4 & $\begin{array}{l}\text { displaced } \\
\text { infiltrated, posteriorly }\end{array}$ & mildly infiltrated, expanded & & mildly infiltrated, & mildly posteriorly \\
displaced, expanded & & & posteriorly displaced & displaced \\
5 & infiltrated & destroyed & normal & normal & mildly infiltrated \\
6 & normal & infiltrated, posteriorly & infiltrated, expanded & infiltrated & normal \\
7 & displaced, expanded & & & & infiltrated \\
\hline
\end{tabular}


Table 4.3 Comparison of median FA and ADC values obtained in the sensory tracts of volunteers and patients.

\begin{tabular}{|c|c|c|c|c|c|c|c|c|c|c|}
\hline \multirow[b]{2}{*}{ Sensory Tracts } & \multicolumn{5}{|c|}{ Left } & \multicolumn{5}{|c|}{ Right } \\
\hline & Pons & Midbrain & VPL & $\begin{array}{l}\text { Internal } \\
\text { Capsule }\end{array}$ & $\begin{array}{c}\text { Subcortal } \\
\text { Sensory }\end{array}$ & Pons & Midbrain & VPL & $\begin{array}{c}\text { Internal } \\
\text { Capsule }\end{array}$ & $\begin{array}{c}\text { Subcortal } \\
\text { Sensory }\end{array}$ \\
\hline \multicolumn{11}{|l|}{ FA } \\
\hline volunteer & 0.661 & 0.515 & 0.646 & 0.559 & 0.585 & 0.689 & 0.503 & 0.636 & 0.623 & 0.552 \\
\hline patient & 0.542 & 0.395 & 0.527 & 0.377 & 0.424 & 0.555 & 0.444 & 0.584 & 0.410 & 0.424 \\
\hline $\mathrm{p}$ value & $<0.001$ & 0.020 & $<0.001$ & 0.003 & 0.007 & 0.005 & 0.036 & 0.027 & 0.003 & $<0.001$ \\
\hline \multicolumn{11}{|l|}{$\mathrm{ADC}$} \\
\hline volunteer & 1.520 & 1.352 & 1.408 & 1.439 & 1.536 & 1.527 & 1.459 & 1.364 & 1.427 & 1.521 \\
\hline patient & 1.704 & 1.626 & 1.736 & 1.450 & 1.608 & 1.752 & 1.488 & 1.612 & 1.482 & 1.592 \\
\hline $\mathrm{p}$ value & NS & 0.011 & 0.024 & NS & 0.044 & 0.01 & NS & NS & NS & 0.047 \\
\hline
\end{tabular}


Table 4.4 Comparison of median FA and ADC values obtained in the motor tracts of volunteers and patients.

\begin{tabular}{|c|c|c|c|c|c|c|c|c|c|c|}
\hline \multirow[b]{2}{*}{$\begin{array}{l}\text { Motor } \\
\text { Tracts }\end{array}$} & \multicolumn{5}{|c|}{ Left } & \multicolumn{5}{|c|}{ Right } \\
\hline & Pons & Midbrain & $\begin{array}{l}\text { Internal } \\
\text { Capsule }\end{array}$ & $\begin{array}{l}\text { Corona } \\
\text { Radiata }\end{array}$ & $\begin{array}{c}\text { Subcortal } \\
\text { Motor }\end{array}$ & Pons & Midbrain & $\begin{array}{l}\text { Internal } \\
\text { Capsule }\end{array}$ & $\begin{array}{l}\text { Corona } \\
\text { Radiata }\end{array}$ & $\begin{array}{c}\text { Subcortal } \\
\text { Motor }\end{array}$ \\
\hline \multicolumn{11}{|l|}{ FA } \\
\hline volunteer & 0.523 & 0.669 & 0.671 & 0.624 & 0.563 & 0.555 & 0.659 & 0.631 & 0.579 & 0.58 \\
\hline patient & 0.300 & 0.595 & 0.662 & 0.619 & 0.526 & 0.353 & 0.628 & 0.664 & 0.546 & 0.490 \\
\hline$p$ value & 0.003 & 0.016 & NS & NS & NS & 0.001 & NS & NS & 0.039 & NS \\
\hline \multicolumn{11}{|l|}{$\mathrm{ADC}$} \\
\hline volunteer & 1.553 & 1.436 & 1.391 & 1.371 & 1.407 & 1.529 & 1.415 & 1.387 & 1.345 & 1.376 \\
\hline patient & 1.666 & 1.664 & 1.448 & 1.420 & 1.488 & 1.822 & 1.570 & 1.428 & 1.420 & 1.518 \\
\hline$p$ value & NS & NS & NS & NS & NS & NS & NS & NS & NS & 0.020 \\
\hline
\end{tabular}

$\mathrm{NS}=$ not significant; $\mathrm{VPL}=$ ventral posterolateral thalamic nucleus. 
concept that a focal axonal insult could produce cellular changes in distant neurons was first described histopathologically by Waller in 1850, and more recently this process has been documented on conventional MR imaging in patients with subacute and chronic stroke [73]. Although we did not detect T2-weighted hyperintensity of corticospinal or sensory tracts in images from our patient cohort, the color maps and FA calculations demonstrated both severe atrophy and reduced anisotropy in the patient in Case 5 (Fig 4.3) prior to any therapy. Diffusion tensor imaging findings of WD have been described in patients who have suffered cerebrovascular accidents $[65,66,74]$. Histopathologically, WD involves both loss of integrity of directional microfilaments in axons and variable buildup of intracellular debris [75]. Thus, one would expect to find both decreased anisotropy and variable diffusivity in this process. Our data corroborated the findings of reduced FA in sensory tracts and, to a lesser degree, in motor tracts in patients with brainstem tumors and variable changes in ADC.

Witwer and colleagues [36] proposed a grading system to clarify supratentorial tract involvement in which they used color maps and FA measures $[32,36,71,76,77]$. Using Witwer et al.'s grading system, 5 of the 7 tumors were classified as diffusely infiltrating and the other 2 tumors had at least 1 focally infiltrative component (Table 4.1). Normalization of the DT imaging data to Talairach space facilitated the selection of ROIs with high probability of sensory and motor connections and improved precision of comparisons between groups. Where as the presence of the brainstem tumors may have limited the accuracy of spatial normalization in the posterior fossa, the color maps helped guide us to the sensory and motor tracts within the tumor itself [68]. Visual inspection of our images located in the supratentorial brain indicated that the sensory and motor tracts were in their expected locations, and we evaluated these tracts to the level of the subcortical white matter.

We found a significant effect of brainstem tumors along distant sensory and motor tracts. Antegrade degeneration or WD in the sensory tracts followed known paths. Tumors of the medulla/pons produced lesions in the second-order sensory neurons as they ascended to synapse with the third order neurons of the ventral posterolateral nuclei of the thalamus [78]. In the motor tracts, FA changes were more pronounced closest to the tumor, with smaller differences in FA values nearer the cortex. Although this may reflect a more heterogeneous local tumor effect on the motor than the sensory tracts (for example, on 1 side significant differences were seen only to the level of the midbrain, and on the other side they were seen to the corona radiata), we cannot exclude the influence of retrograde axonal degeneration [79].

Diffuse pontine gliomas are rarely biopsied prior to therapy [47]. However, focal brainstem tumors are typically approached for biopsy or resection [80,81]. Recent evidence that specific molecular targets may be present in brainstem tumors, [82], has raised the issue of targeted therapy and with it some renewed interest in obtaining tumor tissue to tailor treatment to specific cellular expression in infiltrating brainstem tumors. Roujeau et al.,[83], recently demonstrated that stereotactic biopsy of diffuse brainstem gliomas was associated with minimal morbidity and yielded highly diagnostic tissue samples that would allow precise tumor characterization necessary for participation in 
new treatment protocols. Diffusion tensor imaging coupled with tractography offers the surgeon the opportunity to visualize functional tracts $[68,84]$ to better target areas of tumor outside these important tracts for biopsy and to choose a trajectory to these areas that will minimize the chances of creating new neurological deficits in cases selected for surgery. Presurgical knowledge of the integrity of the sensory and motor tracts may improve the accuracy of prognosis of functional recovery in tumor patients following surgery. In patients with focal tumors in which the tracts are displaced but not infiltrated, recovery of neurological function following either surgical decompression or shrinkage of the tumor by chemotherapy is probable. On the other hand, the recognition of WD in either sensory or motor tracts would predict, even with a good response to treatment, that those tracts and their specific functions would not be recoverable.

Important limitations of this retrospective study include differences in age between the patient and healthy volunteer groups, technical limitations of acquiring DT imaging data in the lower brainstem, a heterogeneous tumor group, and small sample size. Although an age-matched control group is generally the ideal in clinical studies, the median age of our patient group was 7 years. This is well above 2 years, the age of relative stability of FA and ADC values in central white matter pathways [13]; therefore, it is reasonable to assume that the differences in ages between the patient group and control groups in this study did not contribute to differences in the data. Although it is possible that antegrade neuronal degeneration may have been present in motor tracts as well, quantitative analysis of DT images of the medulla was hindered by artifact from the skull base. Additionally, although we cannot sort out the effects of tumor and/or its treatment, the lack of difference between the patient and volunteer groups' FA and ADC at the genu and splenium of the corpus callosum was reassuring that there were not global white matter tract changes in the patient group.

\subsection{Conclusions}

This study demonstrates the potential value in characterizing brainstem tumors by DT imaging and tractography in children. Our data support the hypothesis that FA values are altered proximal and distal to the brainstem tumors, which indicates axonal degeneration in sensory and motor pathways in this patient cohort. These findings are consistent with a growing body of evidence that FA is useful for evaluating axonal degeneration. Although we cannot judge the clinical efficacy or significance of these findings at this time, applications of these techniques are underway in prospective trials at our institution, and these will help to determine the clinical benefit of DT imaging in the management of brainstem tumors in children. The rigorous methods that we used to locate ROIs may improve the accuracy and reproducibility in future investigations of FA changes in other central nervous system disease processes and may provide valuable information in evaluating risks in planning biopsy or debulking of focal brainstem tumors. 


\section{Chapter 5. Utility of DTI in Surgical Planning}

\subsection{Introduction and Overview}

Brainstem gliomas represent $15 \%$ of pediatric CNS malignancies, and until the early 1980s they were regarded as a single pathological entity with a poor Prognosis [31]. Observations of differences in the clinical course among these patients provided evidence, however, that a subgroup of these tumors was resectable and had a better survival rate [85-87]. The introduction of MR imaging provided both a noninvasive multiplanar technique to further distinguish these tumors by precise location and imaging characteristics and an opportunity to correlate these findings with differences observed in the clinical course.

Based on these initial findings, Epstein and Farmer [88] hypothesized that the characteristic growth patterns of low-grade gliomas were determined in part by anatomical barriers in the normal brainstem architecture [85]. The foundation for this hypothesis was based on the morphological investigation of autopsy findings performed by Scherer [89]. It was his contention that, except when destruction of the underlying architecture is more rapid than development of the infiltrating tumor, the normal brainstem architecture plays a more important role in the structure of the glioma than the inherent properties of the glioma itself.

Epstein and Farmer (1993) described four classes of brainstem gliomas characterized by "stereotypical" growth patterns that were easily discernable on MR imaging and could be used, along with clinical data, to establish a reliable diagnosis and therapeutic plan [88]. They proposed that lesions with low malignant potential would present as focal medullary, cervicomedullary, or dorsally exophytic lesions on MR imaging. Conversely, growth patterns of high-grade malignant lesions would be invasive and infiltrative and would smoothly enlarge the affected area without focal exophytic growth.

Although conventional MR imaging techniques are adequate for differentiating tumor tissue from normal tissue, they are not reliable for demarcating the borders of brain-stem gliomas or for demonstrating white matter tract involvement. Diffusion tensor imaging provides remarkable visualization of white matter tracts and is a promising method for evaluating tracts for tumor infiltration to improve diagnosis and surgical planning [90,91]. Although this technique is gaining acceptance for the evaluation of supratentorial lesions, it is only recently being used in the evaluation of the brainstem and spinal cord [92]. We report on two cases in which we investigated the feasibility of using DT imaging for preoperative visualization of the white matter tracts of the brainstem in relation to focal low-grade gliomas, and we describe these findings in terms of the proposal of Epstein and Farmer. 


\subsection{Case Report}

\subsubsection{Case 1}

This 9-year-old girl presented to her pediatrician with progressive snoring and sleep apnea beginning in December 2001. She underwent an adenoidectomy and had moderate transient improvement of her symptoms. Her mother noted phonation changes as well as a return of snoring and apnea, and the patient was reevaluated in October 2002. A laryngoscopy procedure demonstrated partial paralysis of the right vocal cord and a swallowing study indicated uncoordinated pharyngeal muscle activity. Based on MR images obtained at the local hospital and the patient's clinical history, the referring physicians thought that she had a diffuse (intrinsic) brainstem glioma and did not deem that she was a candidate for surgery (Fig 5.1). The girl was subsequently referred to St. Jude Children's Research Hospital for consultation.

At the time of referral, 19 months after the initial onset of symptoms, the patient exhibited bilateral nystagmus with right gaze preference and partial right vocal cord paralysis. No motor strength or sensory deficits were noted; however, cerebellar dysfunction was present. The initial preoperative differential diagnosis was established as a cervical cord tumor as opposed to a brainstem tumor. Diffusion tensor imaging was included in the preoperative imaging examination to help determine if white matter tracts were invaded or disrupted by the tumor because the overlying peritumoral edema blurred the margins of the tumor.

Surgical planning images were obtained on a 1.5-tesla MR imaging unit (Symphony; Siemens Medical Systems, Erlangen, Germany). The imaging examination included a STIR sequence (TR $6440 \mathrm{msec}$, TE $26 \mathrm{msec}$, matrix 512 X 512), lowresolution (FOV 210 X $210 \times 22 \mathrm{~mm}^{3}$ ) and high-resolution (FOV 149 X149 X $22 \mathrm{~mm}^{3}$ ) DT MR imaging sequences (TR $9700 \mathrm{msec}$, TE $127 \mathrm{msec}$, four acquisitions per series, six noncollinear directions, b-1000), [93], and a T1-weighted volume sequence. Diffusion-weighted, echo planar images were acquired using a flexible quadrature surface coil placed around the base of the skull for optimal imaging of the cervicomedullary junction. Saturation bands were placed in the coronal plane, anterior to the basilar pons and medulla to suppress aliasing artifact during the small FOV sequences as well as phase encode artifact from eye movement. Diffusion tensors were calculated using the Statistical Parametric Mapping DT imaging toolbox [41] (http://sourceforge.net/projects/spm-toolbox/) developed for SPM99 (Wellcome Institute of Neurology, London, United Kingdom). Fractional anisotropy, apparent diffusion coefficient, and eigenvector maps were calculated. To aid in the visualization of the fiber tracts for surgical planning, a red, green, and blue orientation colormap was used to demonstrate fiber tract direction [42].

The MR images revealed a cervicomedullary tumor that was mildly hyperintense and partially enhancing on T2-weighted imaging, with a large dorsally exophytic 


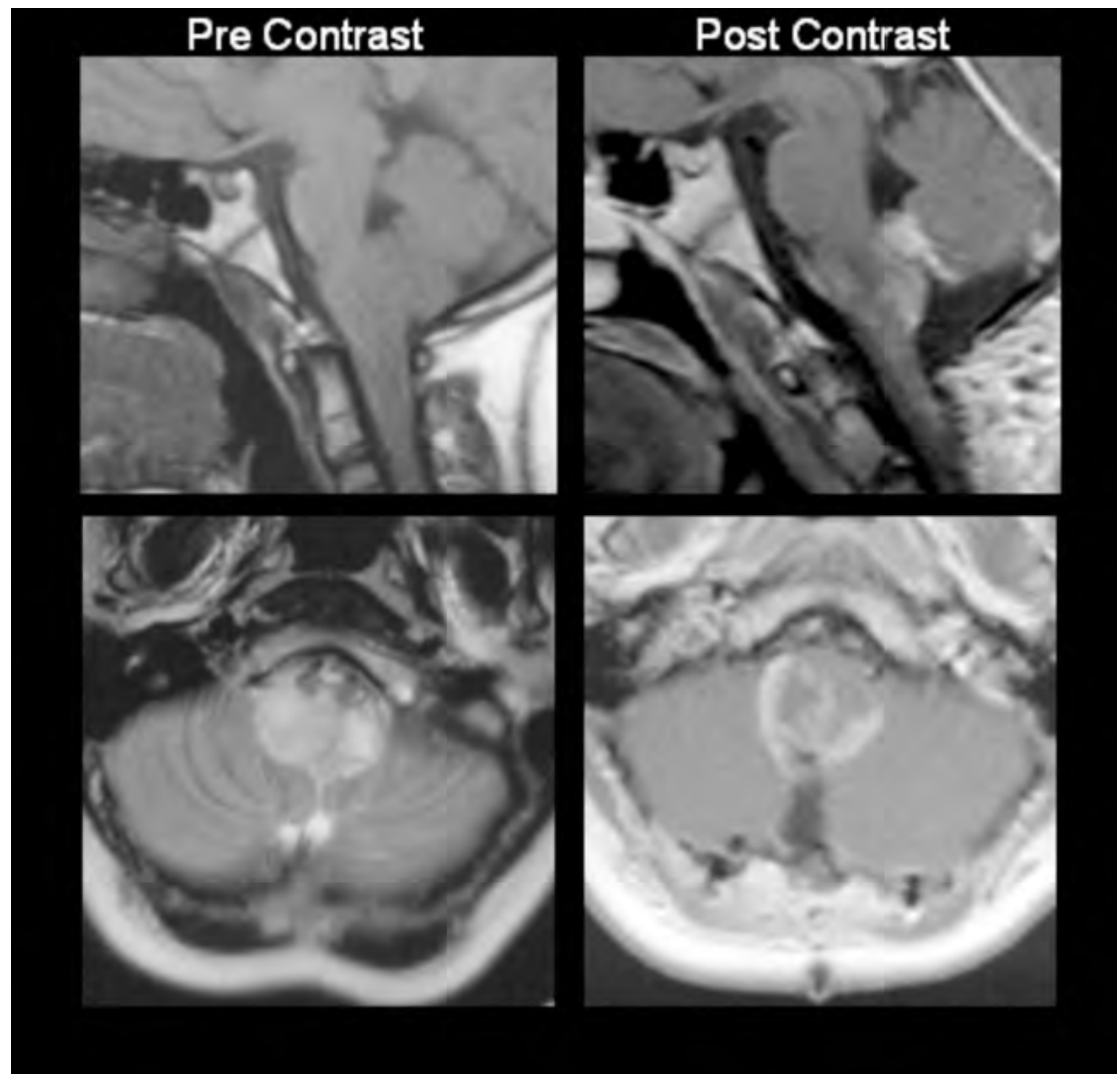

Figure 5.1 Case 1 showing unenhanced and contrast-enhanced T1-weighted sagittal (upper) and axial (lower) images obtained at the referring hospital. A partially enhancing tumor of the medulla and cervical cord is apparent. The neuroimaging findings supported the diagnosis of an invasive glioma of the medulla and cervical cord. 
component extending to the obex and an inferior intramedullary component extending below the level of C-2. Preliminary interpretation of the referral films without DT imaging did not reveal if the cervicomedullary tumor component was diffusely infiltrative or focal. Subsequent comparison of sagittal T2-weighted STIR images used for surgical planning in concert with the DT imaging colormap demonstrated that the signal intensity changes demarcating the borders between tumor tissue and normal tissue by T2-weighted STIR sequences corresponded to regions of tumor surrounded by noninfiltrated white matter fiber tracts of the brainstem and upper cervical cord seen on DT imaging (Fig 5.2 A).

Specifically, preoperative DT imaging demonstrated that caudal tumor growth was cylindrical and well marginated, and that it projected below the pyramidal decussation, to the level of C-2. Cephalic growth was restricted superiorly by the medial lemnisci and projected dorsally toward the obex. Lateral growth was constrained by the spinothalamic and pial fibers in the cervical spinal cord. At the pontomedullary border, lateral growth extended into the middle cerebellar peduncles following the natural path of least resistance. The DT imaging colormap confirmed that the tumor was bound superiorly and laterally by the normal architecture of the brainstem and cervical cord. It also demonstrated that the caudal tumor projection displaced the normal architecture of the pyramidal decussation anterolaterally as it progressed inferiorly to the level of C-2 and occupied the central region of the spinal cord, where the white matter tracts imposed little or no obstacle to growth (Fig 5.2 B).

After a suboccipital craniotomy and C1-3 laminectomy, the dura mater over the cerebellum was opened and a midline myelotomy was performed at the level of C-1 and extended three levels inferiorly. A partial excision of the intraaxial spinal cord tumor was performed, during which the evoked potentials did not change. The dura was closed with a bovine pericardial patch graft and the bone flap was replaced with titanium plates and screws. The patient tolerated the procedure well.

The tumor extended below the obex and descended from the medulla. The upper cervical cord appeared swollen and tense. The intramedullary cervical cord segment of the tumor was well circumscribed and confluent. There was a bridging segment of the tumor dorsal to the cord. At the cervicomedullary junction, the tumor projected from the substantia grisea of the upper cervical cord and exited dorsally. The exophytic component extended superiorly and laterally around the upper portion of the medulla. We believed that it was not prudent to pursue the tumor aggressively at this level.

A gross-total resection of the cervical segment of the tumor and a subtotal resection of the medullary component was achieved. The superior segment of the tumor wrapped around the medulla and could not be safely removed. The patient had transient sensory alterations in her hands and feet from the midline myelotomy but was otherwise intact. She was discharged 5 days postoperatively. Final pathological findings confirmed that the morphological and immunohistochemical features were consistent with a ganglioglioma. 


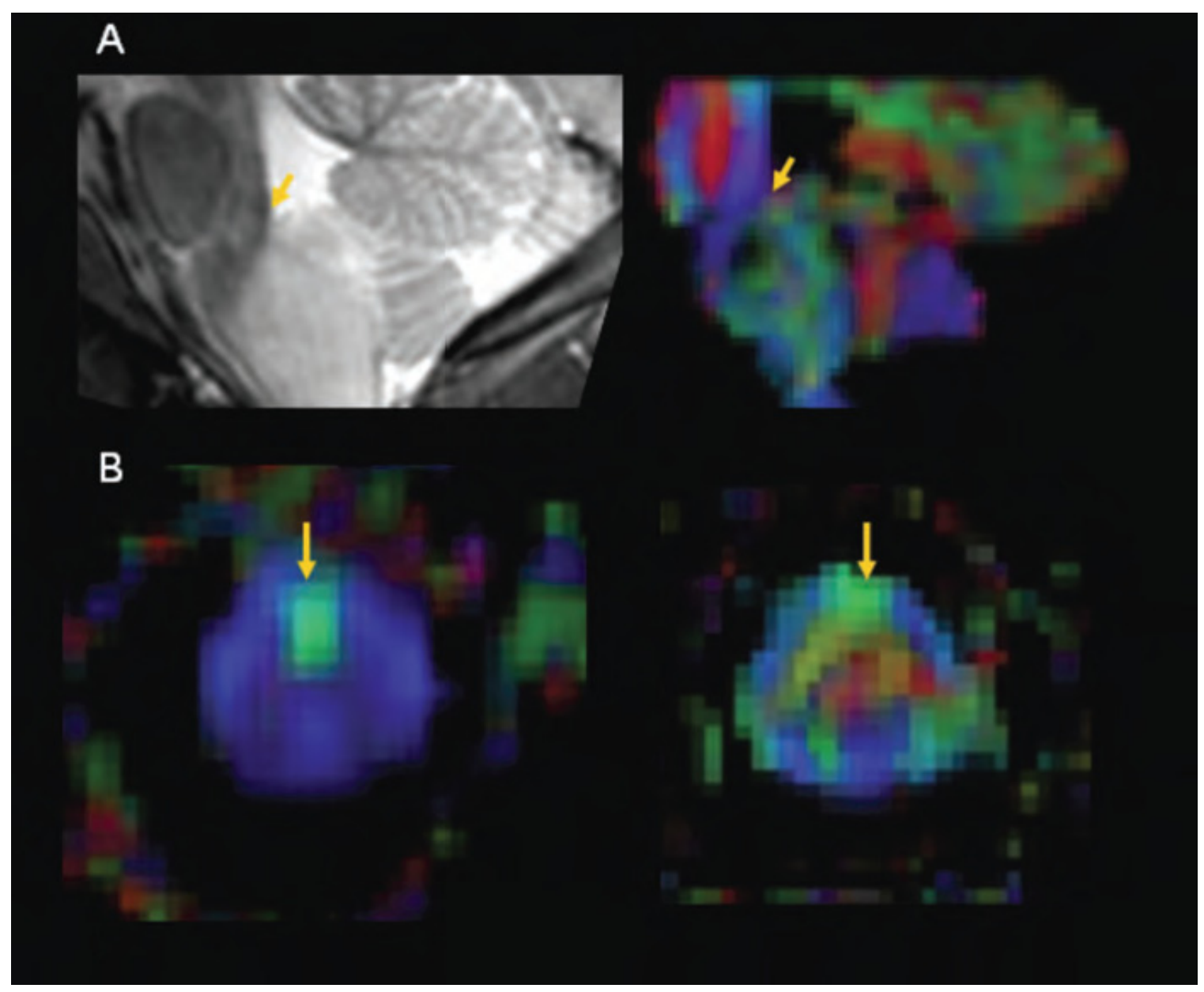

Figure 5.2 Case 1 showing diffusion tensor imaging colormaps. These images demonstrate craniocaudal fibers in blue, left to right traversing fibers in red, and anterior to posterior traveling fibers in green. A) A comparison of preoperative STIR (left) and DT (right) images demonstrates that the changes in signal intensity demarcating the borders between tumor tissue and normal tissue by T2-weighted STIR sequencing corresponding to the medial lemniscus pathway at the pontomedullary junction. $B$ ) Axial DT imaging of the pyramidal tract decussation in an unaffected person (left) compared with the one patient in this case (right) demonstrating that the crisscrossing fiber tract (arrows) was positioned normally and that the tumor had taken up a central location within the cervical cord (in contrast to the apparent diffusely infiltrative appearance on conventional imaging). 


\subsubsection{Case 2}

This 18-year-old woman was referred for evaluation of a recurrent dorsally exophytic brainstem tumor in February 2004. She had initially undergone an incomplete resection of a dorsally exophytic tumor arising from the floor of the fourth ventricle in December 2002. After subsequent recurrence, the tumor was again partially resected, and the patient received radiation therapy in August 2003. Despite these treatments, her symptoms continued to progress and the tumor continued to grow.

At the time of referral, the patient presented with nausea and vomiting, headaches, dizziness, and blurred vision. She had a mild right hemiparesis and a mild left upperextremity hypesthesia. She complained of progressive dysphagia despite maximal steroid therapy. A physical examination revealed poor pharyngeal sensation, a diminished gag reflex, and mild bilateral nystagmus. The patient did not report any facial numbness or pain. An initial preoperative diagnosis was established as a recurrent dorsally exophytic pilocytic astrocytoma.

Surgical planning imaging was obtained on a 1.5-tesla MR imaging unit (Symphony; Siemens Medical Systems). The MR imaging examination included sagittal T1-weighted, axial unenhanced and contrast-enhanced T1-weighted, fluid-attenuated inversion recovery, and T2-weighted images. A three-dimensional magnetizationprepared rapid gradient echo contrast-enhanced sequence with sagittal reconstructions and a low resolution (FOV 230 X 230 X $30 \mathrm{~mm}^{3}$ ) DTI MR sequence (TR $1000 \mathrm{msec}$, TE $100 \mathrm{msec}$, four acquisitions per series, six noncollinear directions, b-1000) were obtained [93]. All images were acquired using a quadrature head coil. Diffusion tensors were calculated and fiber tractography was prepared as previously described.

A dorsally exophytic brainstem glioma arose from the floor of the fourth ventricle and dorsal medulla, extending posteriorly toward the obex and inferiorly into the right foramen of Luschka. The mass was hypointense to the cortex on T1-weighted imaging and hyperintense on T2-weighted imaging. Additionally, the lesion was vividly enhancing on contrast-enhanced imaging. There appeared to be a thin remnant of normal right lateral medulla, which bowed around the mass. This image corresponded to a craniocaudally oriented white matter tract on DT imaging, which was displaced posterolaterally, parallel to the cerebellar peduncles (Fig 5.3).

A suboccipital craniotomy and complete $\mathrm{C}-1$ laminectomy were performed. The dura was dissected from the dural graft patch down to the level of $\mathrm{C} 1-2$. The craniotomy was widened and fresh dural edges were encountered. A careful microdissection of the tumor was performed and a gross-total resection was accomplished. The dura was closed with a bovine pericardial patch. The patient had a persistent tachycardia throughout the surgery, beginning prior to the skin opening, but otherwise she maintained stable vital signs. 


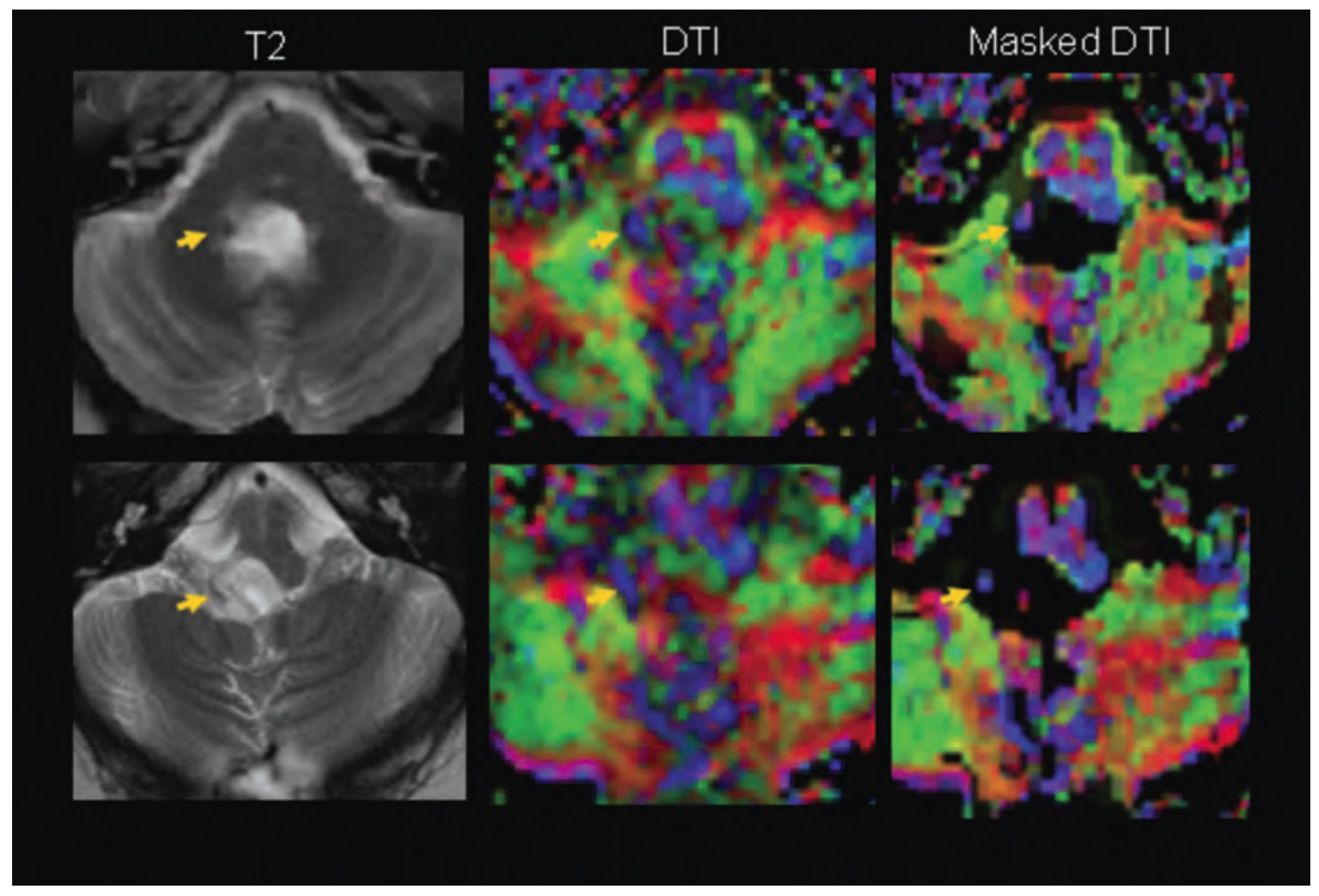

Figure 5.3 Case 2 showing preoperative T2-weighted (left) and raw (center) and masked (right) DT axial images obtained in adjacent slices at the pontomedullary junction. The arrows indicate the correspondence between the fiber tract visualized with DT imaging and the region of normal signal intensity seen on the T2-weighted images. The DT imaging colormaps were masked (trace $<3.5 \times 10^{3} \mathrm{~mm}^{2} /$ second) to suppress color in regions where the diffusion coefficient is higher than in normal tissue to better demonstrate the intact fiber tract, located at the tumor margins (arrows in right). 
On opening of the dura at the C1-2 level, the cerebellar hemispheres and foramen of Magendie were dissected. The tumor could be seen arising from the floor of the fourth ventricle at the obex. The margins of the tumor were circumscribed and could be seen expanding the walls of the medulla. Multiple vessels could be seen perforating the floor of the brainstem and entering the bulk of the tumor. Dissection was performed circumferentially, and care was taken to remove the tumor from the floor of the brainstem. As the dissection proceeded, a plane was created between the brainstem and the floor of the fourth ventricle, and the tumor was completely resected. As observed on the preoperative DT images, a thin band of functional tissue was noted on the right side of the tumor, from which the tumor could be dissected free.

The patient's severe dysphagia continued postoperatively, but no additional cranial nerve deficits were noted. A tracheostomy and feeding gastrostomy were placed, and the patient was discharged home 26 days postoperatively with instructions to take no food or liquids by mouth; however, she died 2 months postoperatively after aspiration of a soft drink.

\subsection{Discussion}

The decision to perform surgery in a patient with a cervicomedullary tumor depends on the clinical history and the focal appearance of the tumor on MR imaging. Surgery is offered only to patients with a focal low-grade tumor and the possibility of gross-total resection or improvement in neurological deficit, because the attendant risk for significant morbidity outweighs the therapeutic benefit of debulking the tumor in high-grade cases. If the clinical history and MR imaging studies conflict on the probability of the tumor being a focal low-grade lesion, surgical treatment is often withheld. This means that some patients who are surgical candidates do not undergo surgery because conventional imaging techniques are unable to differentiate between vasogenic edema and tumor infiltration in the brainstem; therefore, these tumors are misidentified as diffusely infiltrative processes rather than focal low-grade lesions. Consequently, accurate localization of the tumor in relation to important white matter tracts can affect the decision of whether to operate or not, based on the hypothesis of Epstein and Farmer (1987). Using DT imaging in concert with conventional imaging can more accurately demonstrate critical white matter tract infiltration or displacement, and thereby contribute valuable information for the determination of surgical resectability and planning [90].

From a growing series of investigational work, we presented two cases that highlight the potential of DT imaging to identify local white matter tracts in the pons, medulla, and cervical cord and to improve the preoperative assessment of low-grade gliomas. By tracing the white matter architecture of the pontomedullary and cervicomedullary junction in relation to these tumors, it was possible to see that white matter tracts were displaced by the bulk of the tumor but were structurally preserved. This corroborated the prediction of Epstein and Farmer that the underlying white matter 
tracts would "direct" low-grade glioma tumor growth into the stereotypical growth patterns seen on MR imaging.

Both cases demonstrated the improved visualization of white matter involvement in brainstem glioma that can be achieved with DT imaging compared with conventional anatomical imaging. The exact margins of the tumor could not be demarcated using conventional imaging because of the overlying vasogenic edema; therefore, it was difficult to determine whether the tumor was focal or infiltrative at the level of the medulla. With DT imaging, it was clear that the fiber tracts were intact and not infiltrated. These radiological findings were consistent with intraoperative observation and neurological findings that the tumor was well marginated and had caused no focal motor or sensory deficits related to the corticospinal or spinothalamic pathways.

The findings obtained in Case 2 also demonstrated the ability of DT imaging to provide crucial information to the neurosurgeon about the contours of the tumor. In particular, it predicted that the tumor did not infiltrate the spinal tract of the trigeminal nerve that was stretched around the lateral margin of the tumor (Fig 5.3). This finding correlated with the neurological examination, during which no deficits of taste, pain, or light touch sensation to the face were found; rather, it revealed markedly decreased sensation of the pharynx, as well as a diminished gag reflex. This trigeminal tract was not well visualized on conventional imaging and, prior to the DT imaging examination; the band was interpreted as a pial boundary surrounding the tumor. Based on the conventional imaging alone, the tract may have been included in the resection. The DT images clearly demonstrated the presence of a coherent band of functional tissue. In contrast to the focal lesion seen in Fig 5.3, a diffusely infiltrative pontine glioma appears on DT imaging to completely infiltrate and distort involved white matter pathways (Fig $5.4)$.

The DT imaging directly influenced the presurgical planning for this case, by alerting the neurosurgeons to the presence of functional tissue at the lateral borders of the tumor. As a consequence, the patient was spared a complete resection of the involved pathway and an additional postsurgical deficit. We note that effective tests for continuous intraoperative neurophysiological monitoring in the brainstem are limited. Specifically, neurophysiological tests for the afferent branch of the swallowing reflex are still lacking. The DT imaging approach may offer a temporary solution to this problem by providing preoperative mapping of internuclear fascicles.

Diffusion tensor imaging is being used widely to evaluate peritumoral regions in the cerebellum and cerebrum; $[32,35 ; 36]$ however; the application of this technique to the brainstem and to brainstem lesions is limited. We found no reports of DT imaging being used to characterize brainstem pathological features [92,94]. Further work is needed to validate this technique for the wide range of brainstem tumors that occur in children. In addition, quantitative parameters of water diffusion in the brain, such as fractional anisotropy and apparent diffusion coefficient, may help to improve diagnostic evaluation and surgical planning for pediatric brainstem gliomas, beyond the improved visualization demonstrated in this report [32]. 


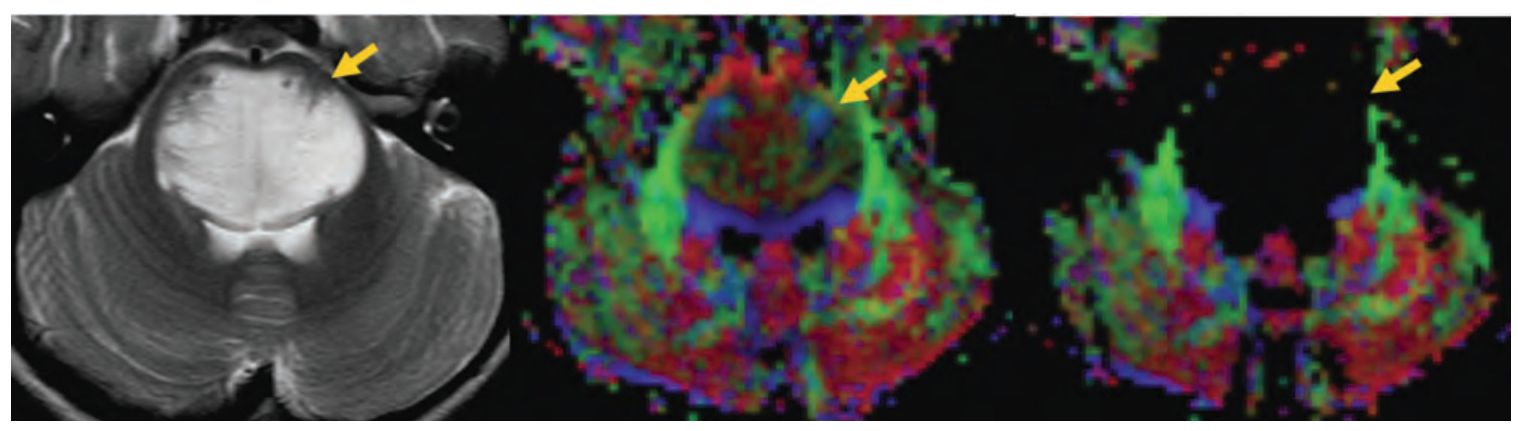

Figure 5.4 Conventional and DT images obtained in a 9-year-old female patient with a diffusely infiltrative pontine glioma. Axial T2-weighted image (left) and unmasked (center) and masked (right; Trace $<3.5 \times 10^{3} \mathrm{~mm}^{2} /$ second) DT images demonstrate that the pyramidal tracts (arrows) are infiltrated and reticular fibers posteriorly displaced and thinned. Both tracts showed markedly diminished FA values, consistent with infiltration.

\subsection{Conclusions}

Diffusion tensor imaging is a useful in demonstrating the relationship between tumor tissue and normal brainstem white matter architecture, and for improving the surgical evaluation and management of pediatric brainstem tumors. 


\section{Chapter 6. Evaluation of Medulloblastoma Therapy with DTI}

\subsection{Medulloblastoma: Overview and Contemporary Therapy}

Medulloblastoma is a subclass of Primitive Neuroectodermal Tumors (PNET) that arises exclusively in the cerebellum. The origin of these cell lines is uncertain although the World Health Organization (WHO) classifies them as embroynal tumors [95]. It is the most frequent malignant brain tumor in children and is uniformly fatal in untreated patients. The peak incidence is between 5 and 8 years of age, with a full third occurring before 3 years of age [95]. These tumors typically arise in the vermis of the cerebellum and often compromise CSF flow through the $4^{\text {th }}$ ventricle. Medulloblastoma are highly malignant tumors that preferentially metastasize to the spinal cord via subarachnoid pathways, though $5 \%$ of patients may present with bone or lymph node involvement. Patients with ventriculoperitoneal shunts have increased risk for peritoneal metastasis.

The current approach to therapy is to assign the child to a risk group based on the Langston modification of the Chang staging for medulloblastoma [95] (Table 6.1). Children in the T1-T3 disease are considered standard risk. T4-M4 classified as high risk. Children with less than $1.5 \mathrm{~cm}^{3}$ of residual tumor are considered standard risk. With contemporary treatment and the absence of metastasis at diagnosis, 5 year survival can be as high as 80 percent with at least 50 percent of children surviving until adulthood [96]. Poor prognostic indicators include age ( $<4 \mathrm{yrs}$ of age), sex (male), extent of disease (leptomeningeal disease), and incomplete surgical resection. The key to long-term survival has been the combined treatment of maximal surgical resection followed by whole-head irradiation with $35 \mathrm{~Gy}$, a posterior fossa boost of $15 \mathrm{~Gy}$, and spinal cord radiation of 35-55 Gy [97]. These tumors are highly malignant and disseminate along the neuroaxis. Fortunately, medulloblastomas are exquisitely radiosensitive and are less likely to recur following brain and spine irradiation.

\subsection{Neurocognitive Sequelae of Medulloblastoma Therapy}

Unfortunately, the majority of these long-term survivors will face life long learning problems and diminished IQ levels. Studies done at St. Jude Children's Research Hospital have noted that damage to healthy neural tissue is correlated with diminished neurocognitve facility and intelligence test score $[13,14,19,98]$. Operative morbidity is reported to occur in approximately $40 \%$ of cases. These post operative sequelae include posterior fossa syndrome of mutism (also known as cerebellar mutism), pharyngeal and respiratory dysfunction and ataxia [99]. Most of these problems resolve in the months following surgery [100]. However, the neurocognitive consequences of cranial irradiation in children, including disturbances in attention, memory and reading, do not appear to improve with time. As part of an institutional study, survivors of acute lymphoblastic leukemia (ALL) and pediatric brain tumors underwent fMRI studies of the primary visual cortex. All subjects had received either total cranial radiation or CNS directed chemotherapy or both. When compared to healthy siblings, recipients of cranial 
Table 6.1 Langston modification of Chang staging for medulloblastoma.

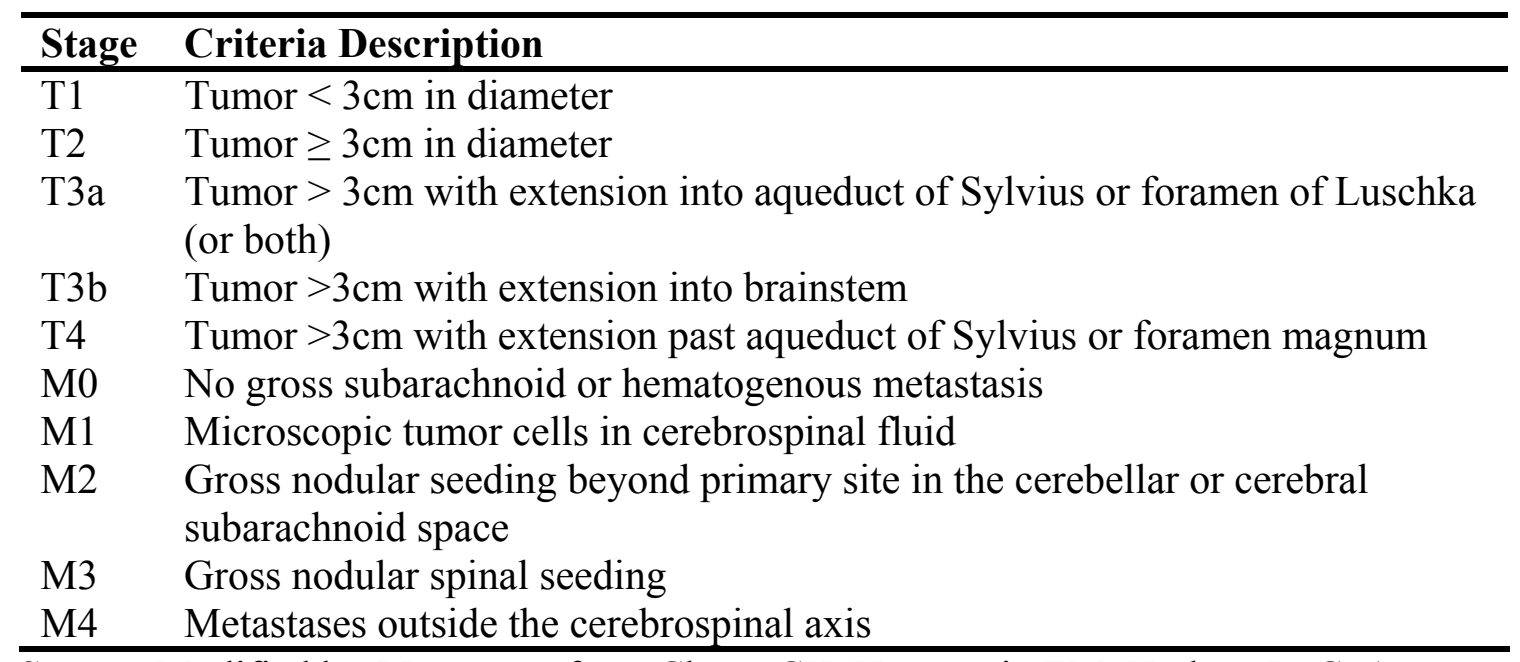

Source: Modified by J Langston from Chang CH, Housepain EM, Herbert Jr. C. An operative staging technic for cerebellar medulloblastomas. Radiology 1969;93:13511359.

RT were associated with smaller volumes of activation in the occipital lobe [101]. Also as part of this series, all subjects underwent fMRI examination using the Conner's Continuous Performance Task (CPT) [102]. The continuous performance task has been shown to be sensitive to brain injury and is most commonly used in the evaluation of Attention Deficit Hyperactive Disorders (ADHD) [103]. In our study, we found significant differences in the behavioral performance and brain activation of survivors compared to healthy controls. Significantly, we found decreased laterality of ventral visual activation in survivors.

The current goal of therapy is to increase survival of these children while decreasing the neurocognitive sequelae associated with standard therapy. In keeping with these goals, St. Jude Children's has been investigating combination chemotherapy and reduced dose CSI in patients. The institutional protocol SJMB03 is a study open to children classified with high-risk disease. "Therapy includes gross total surgical resection, stage related craniospinal irradiation, and 4 cycles of dose intensive chemotherapy followed by stem cell rescue" (Table 6.2) [104].

\subsection{The Ventral Visual System and Orthographic Processing in the Human Brain}

Reading is an acquired skill that involves the fundamental processes of mapping visual (orthographic) input onto auditory (phonologic) and conceptual (semantic) representations in the brain (RO1-grant proposal). A growing body of evidence suggests that a predominately left-lateralized neural network involving posterior superior temporal, inferior parietal, inferior frontal, extrastriate occipital, and occipotemporal areas is involved in normal reading activity [105-110]. Here we focus on orthographic 
Table 6.2 SJMB03 treatment schemes based on risk assessment.

\begin{tabular}{|c|c|c|c|c|}
\hline \multirow[t]{2}{*}{ Staging } & \multicolumn{2}{|c|}{ Radiotherapy } & \multirow[t]{2}{*}{ Chemotherapy } & \multirow{2}{*}{$\begin{array}{l}\text { Duration } \\
\text { of } \\
\text { treatment }\end{array}$} \\
\hline & $\begin{array}{c}\text { Cranio- } \\
\text { spinal }\end{array}$ & $\begin{array}{l}\text { Posterior } \\
\text { fossa }\end{array}$ & & \\
\hline \multicolumn{5}{|l|}{ Avg risk } \\
\hline T1-3B, M0 & 23.4Gy & $54 \mathrm{~Gy}$ & $\begin{array}{l}\text { Vincristine, lomustine, and } \\
\text { cisplatin }\end{array}$ & $56 \mathrm{wks}$ \\
\hline $\mathrm{T} 1-3 \mathrm{~B}, \mathrm{M} 0$ & 23.4Gy & $55.8 \mathrm{~Gy}$ & $\begin{array}{l}\text { Vincrisitine, cyclophosphomide, } \\
\text { cisplatin }\end{array}$ & $56 \mathrm{wks}$ \\
\hline T1-2B, M0 & 25Gy & $55 \mathrm{~Gy}$ & $\begin{array}{l}8 \text { drugs in 1day or carboplatin and } \\
\text { etoposide }\end{array}$ & $21 \mathrm{wks}$ \\
\hline \multicolumn{5}{|l|}{ High risk } \\
\hline M1-3 & 36Gy & $55.8 \mathrm{~Gy}$ & Vincristine, lomustine, cisplatin & $56 \mathrm{wks}$ \\
\hline M2-3 & $36 \mathrm{~Gy}$ & $54 \mathrm{~Gy}$ & $\begin{array}{l}8 \text { drugs in } 1 \text { day or weekly } \\
\text { vincristine }\end{array}$ & $56 \mathrm{wks}$ \\
\hline M2-3 & $35 \mathrm{~Gy}$ & $55 \mathrm{~Gy}$ & $\begin{array}{l}\text { Vincristine, etoposide carboplatin, } \\
\text { or vincristine, etoposide, } \\
\text { cyclophosphamide. }\end{array}$ & 23 wks \\
\hline M2-3 & $35.2 \mathrm{~Gy}$ & $55.2 \mathrm{~Gy}$ & $\begin{array}{l}\text { Ifosfamide, cisplatin, etoposide; or } \\
\text { cytarbine, methotrexate. }\end{array}$ & 56 wks \\
\hline
\end{tabular}

processing via the ventral visual pathway and review the literature on the role of the ventral visual pathway in normal and abnormal reading.

The initial neural process in reading requires a transformation of the retinotopic representation of text in the primary visual cortex into representations that can be recognized independently of location, size, font, etc., and mapped onto corresponding phonologic and semantic representations. Object recognition, including letters and words, occurs in the ventral visual pathway [111,112]. Visual information processing proceeds from the primary visual cortex, through extrastriate visual areas, toward association areas in the temporal lobe and temporoparietal area; neural representationsprogress from discrete retinotopic object features (e.g., form and color) to categorical and individual objects (e.g., faces, letters, or words). Some categories of objects, notably faces, letters, and words, appear to engage specific areas of the brain with reproducible location across individuals. These category-specific sites are often lateralized to one hemisphere of the brain [111].

Orthographic recognition (letters and words) in mature readers is lateralized to the left hemisphere in a location that is nearly symmetric to the Fusifrom Face Area (FFA) [107,111,113-119]. Cohen and colleagues have proposed a dedicated visual word-form area in the left fusiform gyrus [114-116,120], and though the specific computational role of this area is controversial [118,121-123], its importance in orthographic processing is well established. Because of this lateralization, whole-object recognition generally requires integration of information from both the left and right visual fields, so the 
development of word and letter recognition in the left hemisphere requires transcallosal white matter connections between visual areas performing basic feature analysis [120,124-126]. This is a key focus of our research, in light of our finding of abnormal white matter connections development in patients with medulloblastoma [127].

Eden and colleagues showed that the development of reading skill is associated with increasing left lateralization of the extrastriate visual activity [119]. This crosssectional study used an implicit reading paradigm [107] with a simple feature-detection task to avoid performance confounds among subjects aged 6 to 22 years. Increased lateralization in extrastriate visual areas resulted from decreased activation in the right hemisphere; left hemisphere activation was unchanged. The authors suggest that young children use simple feature-based analysis to recognize orthographic stimuli. Then as expertise develops, word and letter recognition becomes connected with phonologic and semantic representations via the ventral visual pathway in the left hemisphere. In contrast to findings of Eden, Schlaggar, et al. reported developmental increase in left ventral visual activation during processing of single words in the context of a wordgeneration task [128]. Left-hemisphere activity in this region was remarkably constant, while right-hemisphere activity increased monotonically with decreasing word-form familiarity [129]. Thus, lateralization of word and letter recognition in the left ventral visual pathway is an important step in the development of normal reading skill, and studies in clinical populations have shown altered activation in this pathway in subjects with reading disabilities.

\subsection{Radiation Dose Distribution in Medulloblastoma Patients}

The effects of radiation on the cerebral vasculature are well documented and include fibrous wall thickening, thrombosis, and luminal occlusion. These changes lead to alterations in blood perfusion, astrocyte enlargement and fluid accumulation. At doses above 50Gy in the brain, this can lead to severe radiation necrosis. To better understand the role of radiation on neurocognitve changes, radiation dosimetry maps from the SJMB96 protocol were combined with MRI images. The fused image sets demonstrated that the splenium of the corpus callosum is disproportionately affected by radiation therapy in medulloblastoma survivors (Fig 6.1). This suggests that the altered patterns of brain activity in the ventral visual pathway may be related to a radiation insult to otherwise healthy nervous tissue [130-132].

\subsection{DTI in Evaluation of Therapy-Induced Changes}

In summary, neurocognitive sequelae with current therapeutic modalities reduce the quality of life for survivors of medulloblastoma and a number of other pediatric and adult cancers. The ventral visual pathway has an essential role in supporting normal reading in the human brain. Thus, the medulloblastoma survivors that we studied served as a model population for acquired dyslexia related to well-defined therapeutic insult. 

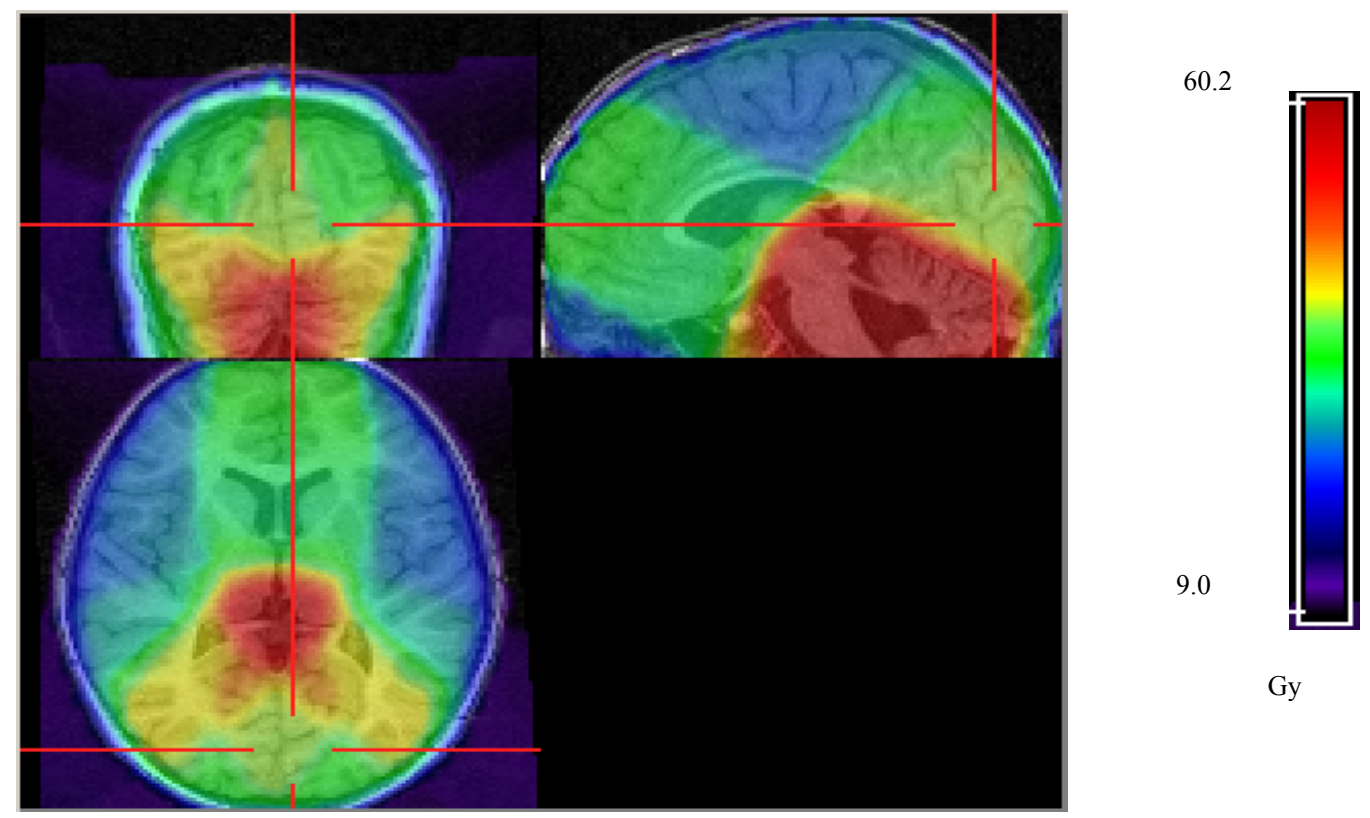

Gy

Figure 6.1 Combined MRI and radiation dosimetry map shows that the splenium of the corpus callosum is disproportionately affected by the radiation therapy. Additionally, white matter pathways associated with the optic radiations and ventrovisual pathway receive greater amounts of radiation than other supratentorial white matter tracts. [133]

The longitudinal imaging studies we performed in patients yielded new insights into the role of the ventral visual system in development of abnormal reading ability. In addition, although our previous results pointed to a reading deficit, it is crucial to our understanding of neurocognitive effects of therapy in patients with cancer to determine the extent to which these problems are selective to reading or whether they affect other visual processing functions supported by cortex.

This study uses Diffusion Tensor Imaging (DTI) to investigate functional anatomy and connectivity in the extrastriate ventral visual pathways and to characterize the relationship in regards to acquired reading deficits and therapy-induced brain damage in pediatric medulloblastoma survivors. We hypothesized that behavioral deficits in reading decoding among medulloblastoma survivors reflect therapy-induced disruption of the maturation of ventral visual processing during the development of reading skills. In analysis of the FA we can assume that there is no longitudinal change in healthy subjects. This allows us to simplify our analysis since age at diagnosis does not enter the model. For patients diagnosed and treated at any age, the FA is starting at approximately the same point. After treatment, we hypothesized that FA would decline because of therapyinduced injury. 


\subsection{Experimental Design and Methods}

We report a longitudinal experimental study to obtain DTI information to test our hypothesis that radiation therapy will produce transient changes in FA and ADC in medulloblastoma survivors. Subjects received serial DTI examinations at 3 month intervals over a 1 year period. All subjects were evaluated at no less than 3 time points during the one year period. Voxelwise analysis of multisubject diffusion date was conducted using Tract Based Spatial Statistics (TBSS) using the FSL software package (Oxford Centre for Functional Magnetic Resonance in the brain, (www.fmrib.ox.ac.uk/fsl) to determine global differences in FA [134].

Subjects were recruited from patient groups comprised of patients diagnosed with medulloblastoma treated at St. Jude since 1996. The extrastriate visual ROIs in this proposal are adjacent to the posterior fossa; therefore, they receive higher, more variable doses of radiation than they do in children with supratentorial PNET. These patients do not have tumor or surgical lesions that might directly affect supratentorial neural systems involved in reading.

All subjects were at least 6 years old at the time of their first evaluation, were enrolled in school or homebound instruction, and was were English-speaking. Specific exclusion criteria include the following:

- Estimated IQ less than 50.

- History of CNS injury or disease (controls) or CNS involvement predating cancer (patients).

- History of documented ADHD, Attention Deficit Disorder, or Learning Disability (controls) or similar history predating cancer (patients).

- Treatment with psychostimulants (e.g., Cylert, Ritalin, or other drugs intended to improve cognitive functions) or psychotropic medications intended to treat mood or thought disorders within the 2 weeks before either MRI or neuropsychological testing (patients or controls).

- A major physical, neurological, or psychiatric condition (such as psychosis, blindness, paresis, poorly controlled seizures) that would preclude participation in the research program (patients or controls). Such a condition will be determined by medical, neurological, and psychiatric reports in the medical chart (patients) or via report from parent or guardian. The functional criteria for exclusion are an inability to perceive and comprehend psychological testing materials or to comply with testing instructions.

- Orthodontic appliances that cause image distortion or signal loss outside the mouth and sinus area.

Eighty patients were enrolled at the time of analysis. The range of age at the time of DTI was 6 to 25 years, that of age at diagnosis was 3 to 18 years, and that of time since completion of therapy was 1 to 10 years. The age at time of DTI or time since diagnosis could advance by up to 2 years for subjects enrolled later. 


\subsection{DTI Studies}

The DTI data was acquired using bipolar diffusion-encoding gradients to reduce gradient-induced eddy currents that cause image distortion and degradation [93,135]. All images were acquired on our 1.5-T Siemens Symphony scanner using a double spin echo EPI pulse sequences with the following parameters: TR, $10.0 \mathrm{~s}$; TE, $100 \mathrm{~ms}$; FOV, 192 mm; matrix, 128 x 128; slice thickness, $3 \mathrm{~mm}$. Gradient pulses for diffusion weighting are characterized by a quantity, $b$, which as described in Chapter 2, is a function of the gradient strength and the timing of the gradient pulses. Seven images, 1 in which $b=0$ and 6 in which $\mathrm{b}=1000 \mathrm{~s} / \mathrm{mm}^{2}$, are used to calculate the diffusion tensor for each voxel. For the $b=1000$ images, the diffusion gradients will be applied along 6 non-collinear, non-coplananer directions in space defined by turning on the $\mathrm{x}, \mathrm{y}$, and $\mathrm{z}$ gradients pairwise as follows (x y z): $110,-110,101,10-1,011$, and $0-11$. The set of 7 images were acquired 4 times. Image realignment and reslicing were performed with the DTI toolkit (http://sourceforge.net/projects/spmtools) under SPM2. Data from the 4 acquisitions were realigned before tensor calculation to correct for linear image drift, which is caused by gradient-induced heating. Realignment parameters were estimated for the $b=0$ images of each acquisition and then applied to all of the diffusion-weighted images for the respective acquisition. The diffusion tensor was solved using a weighted linear least-square fit implemented using the DTIfit algorithm as part of the Functional Magnetic Resonance Imaging of the Brain (FMRIB's) diffusion toolbox (FDT v2.0) in the FSL software package (Oxford Centre for Functional Magnetic Resonance in the brain, (www.fmrib.ox.ac.uk/fsl). Voxel wise statistical analysis was then conducted using Tract Based Spatial Statistics (TBSS, v1.2) [134].

\subsection{Image Analysis}

Pre and post operative imaging was performed on 6 patients. Three month interval imaging was completed on 80 (59 male, 21 female) patients, 6 month imaging completed on 63 patients, 9 month imaging completed and 1 year imaging on 55 patients. Five patients were removed from the final analysis due to metal artifact and poor quality imaging. Thus, 50 patients (40 male, 10 female) comprised the final cohort.

Diffusion tensor imaging preprocessing was performed using the Functional MRI of the Brain (FMRIB) Software Library (FSL) (FMRIB Centre, University of Oxford, Oxford, United Kingdom). Fractional anisotropy (FA) images from the immediate postoperative scan were calculated from the diffusion tensor in each voxel. The tract-based spatial statistics (TBSS) tool in FSL was used to identify tract-based differences in FA values between the CMS and control patients. Tract-based spatial statistics computes a group mean FA skeleton, which represents the centers of all fiber bundles that are common to the subjects involved in the study. The aligned FA image for each subject was projected onto the tract skeleton. The skeletonized FA maps for the groups were compared with the non-parametric permutation procedure called randomize in FSL. Differences in FA between the groups were considered statistically significant for $\mathrm{p} \leq$ 0.05 , after correction for multiple comparisons [134]. 


\subsection{Results}

All patients analyzed were diagnosed with medulloblastoma at the time of their initial imaging and subsequently verified by surgical pathology. Patients were similar with respect to treatment risk, extent of surgical resection, and timing of all scans. However, there was a significant (4:1 ratio) predominance of males to females in the final cohort. A review of several published series reveals that the male-to-female ratio is reported as 2-4:1 and this variation may represent a true male preponderance of this disease $[136,137]$.

On the immediate post-operative scan, no significant differences were seen in the FA of the skeletonized tracts by TBSS after correction for multiple comparisons. Additionally, three month and 6 month interval scans demonstrated no significant differences in white matter FA when compared to baseline scans. Region of interest examinations were performed of the splenium of the corpus callosum to determine if any trends in mean FA could be seen (Fig 6.2). Eight subjects were selected at random and ROI's were manually placed in the splenium of the corpus callosum for each 3 month interval. The FA's were normalized and plotted. This analysis revealed that though FA changes were occurring during treatment, there was wide variance in the normalized FA values. And although normalized FA appears to decrease for individual subjects, a linear regression analysis revealed no significant trend $\left(\mathrm{R}^{2}=0.0005\right)$.

Interestingly, however, by the nine and 12 month interval patients demonstrated significantly reduced FA $(\mathrm{p}<0.05)$ in four areas: 1) Bilateral pulvinar, 2) Gyrus cinguli, 3) Bilateral corona radiata, and 4) Bilateral corticospinal tracts. Additionally, 12 month interval vs baseline also displayed significantly improved FA $(\mathrm{p}<0.05)$ in three areas: 1$)$ The splenium of the corpus callosum, 2) The genu of the corpus callosum, and 3) Right middle temporal lobe.

\subsection{Conclusions}

Several interesting findings presented itself in the final analysis of the SJMB03 data. The easiest to elucidate is the findings of the reduced FA seen in the corticospinal tracts and corona radiata. These findings mimic those discussed in Chapter 4 where we demonstrated the loss of anisotropy in distal white matter pathways correlated with focal insults in brainstem structures. As such, these changes in FA most likely represents the evolution of axonal degeneration changes in the distal motor and sensory pathways of brain stem structures that have received full dose radiation. More difficult to explain is the decrease seen bilaterally in the gyrus cinguli. The cingulate dorsally surrounds the corpus callosum and is divided into anterior and posterior regions. Changes seen in our study are localized to the anterior cingulate region. Interestingly, the gyrus cinguli receives projection fibers from the medial temporal lobe. More specifically, it is closely associated with the parahippocampal gyrus, [138], and harbors numerous fiber bundles that connect the neocortex with medial temporal structures, such as the hippocampus. 


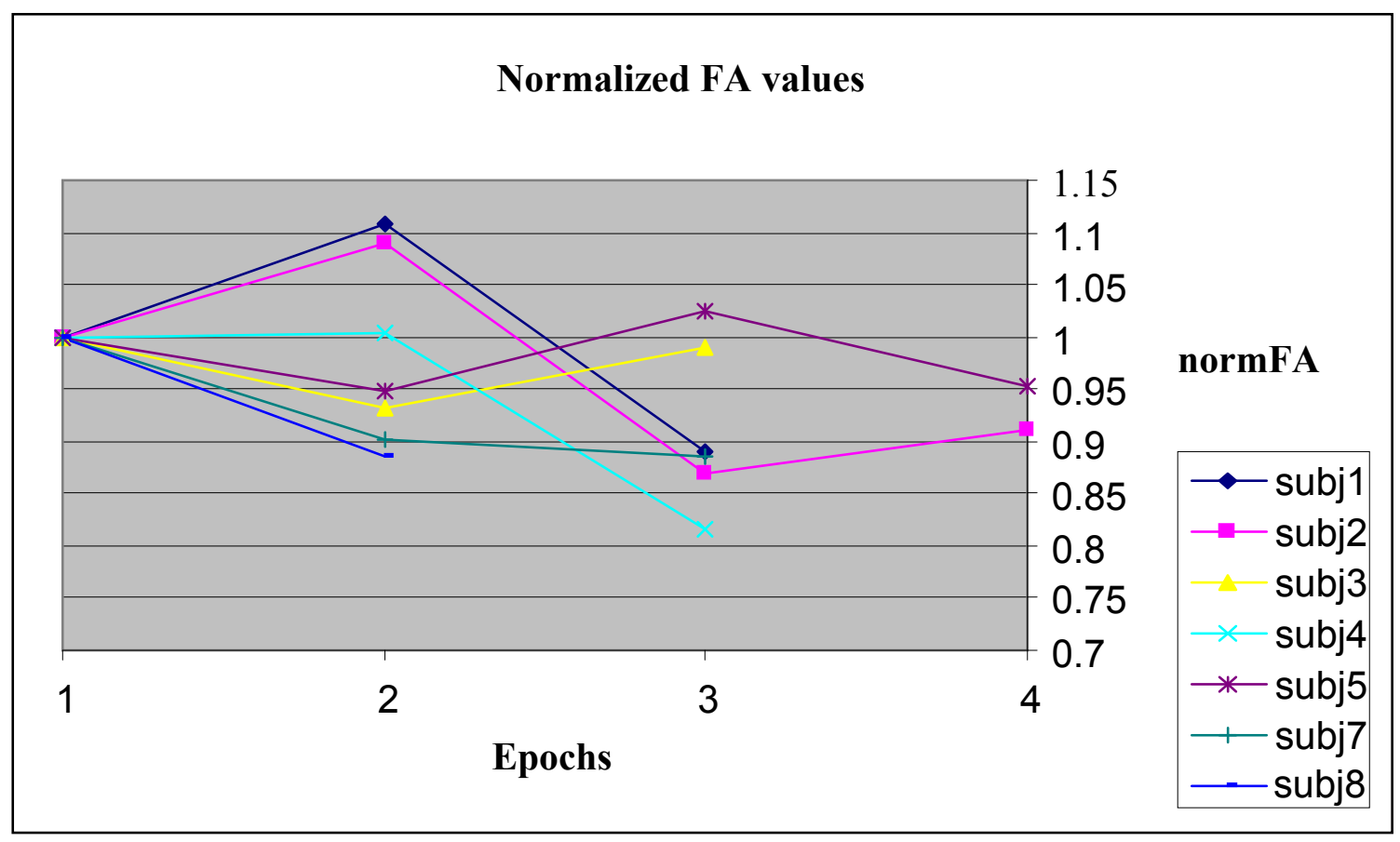

Figure 6.2 Example of time course of FA changes of a single ROI of the splenium in three month intervals over one year for 8 random subjects.

Given this relationship, it becomes plausible to hypothesize that FA changes seen in the cinguluate may represent distal axonal degeneration changes in the middle temporal lobe. This explanation is confounded by the fact that TBSS analysis demonstrated that the FA in the right middle temporal lobe improved after one year when compared to baseline $(\mathrm{p}<0.05)$. Thus changes seen in the middle temporal lobe should lead to an improvement in FA rather than a decline. Thus the changes in the middle temporal lobe may in fact contribute little to the overall FA seen in the cingulum. Additionally, both the genu and splenium of the corpus callosum demonstrated improved FA and would argue against changes in regional association fibers such as those that stream in from the posterior parietal cortex or association fibers which are seen in significant numbers $[139,140]$. Postmortem studies in humans, have demonstrated that the hippocampul component of the cingulate and parahippocamal is poorly developed [141]. Recent studies have revealed the presence of very large pyramidal neurons in the anterior cingulate gyrus that play an important part in executing voluntary motor and premotor controls [142-144]. Given this association, changes in FA seen in the corticospinal and corona radiata, may manifest in this region due to the presence of these corticospinal projections and would explain the reduced FA demonstrated by TBSS.

Until recently, it was thought that the vast majority of visual information and visual attention was purely a cortical phenomenon [145]. However, a growing body of research indicates that the Lateral Nuclear Group (LNG) of thalamic nuclei might play a key role in directing visual attention [146-148]. The pulvinar, the largest group of nuclei of the LNG, is strongly indicated as having a modulatory role in directing visual 
attention. Visual information from the retina passes through the thalamic relay via the lateral geniculate nucleus. These connections pass through the LNG and are parsed in an organized and spatiotopic fashion [145]. Anatomical studies have demonstrated that the pulvinar contains organized retinotopic maps $[149,150]$. And projections from the pulvinar connect to the primary visual cortex (V1) and extrastriate visual areas (V2 and V4), and middle temporal visual area (MT) as well as the frontal eye fields and limbic areas $[149,151,152]$. This suggests that the pulvinar plays a key role in directing visual information and theoretically plays a role in visual attention.

Kastner and Ungerleider (2000), demonstrated that the pulvinar is part of a network controlling visual spatial attention [153]. Karnath, et al. (2002), demonstrated that patients with lesions in the pulvinar exhibited visual spatial neglect [154]. Concurrently, Kastner, et al., (2004), reported functional activation during an fMRI attention paradigm [147]. During the behavioral task the pulvinar was consistently activated across subjects and was strongly modulated by selective attention.

Given this evidence we strongly suspect that the significant decrease in FA in the pulvinar seen in the medulloblastoma patients at one year represents evidence for treatment induced neurotoxicity associated with visuospatial attention deficits. Several studies have demonstrated that white matter anisotropy is associated with neurocognitive function [65,155]. Nagy et al. (2004), demonstrated white matter maturation correlated with neurocognitive development [156].

In our cohort of pediatric medulloblastoma survivors, we have been able to exclude the primary effects of tumor, tumor resection and post operative complications such as posterior fossa syndrome and hydrocephalus. Therefore we speculate that these changes represent white matter damage from cranial radiation and chemotherapy. Unfortunately, our study was unable to detect changes in the shorter intervals of the treatment regimen, and therefore is unable to determine the contribution of radiation and chemotherapy alone in the decline in FA seen in the pulvinar. Our findings therefore can only be considered to be sensitive to the locus of the injury rather than give insight into the mechanism of the injury. However, we theorize that based on the radiation therapy maps that radiation exposure plays a key role. Radiation dose maps for our cohorts demonstrate the pulvinar routinely is exposed to the same dose delivered to the primary tumor site. While chemotherapy, which can affect cognitive ability, is not know to be preferentially absorbed to the thalamus or its substructures [157]. Specifically, vincristine has been detected in the parietal cerebral cortex of animal models, however, the levels detected were much lower than those predicted based on pharmacological models. As a consequence, we predict that in general any effects seen from chemotherapeutic agents would be distributed according to steric hindrance and low permeability at the bloodbrain barrier. And as a consequence we would see cortical and generalized distribution which would manifest as a nonspecific decrease in FA and non-specific local changes. 


\section{Chapter 7. DTI in Cerebellar Mutism}

\subsection{Introduction}

Under normal circumstances, the cerebral cortex and cerebellum are in circuit via afferent and efferent signaling. Impulses originating within the cerebral cortex travel along axons via the corticopontocerebellar pathway to ultimately terminate within the Purkinje cell layer of cerebellum. Axons from these neurons form inhibitory synapses onto the deep cerebellar nuclei. Subsequent excitatory projections from these nuclei to various targets within the spinal column, brainstem, and cerebral cortex are well recognized for their importance in affecting coordination of movement. The efferent pathway which ultimately reciprocates with the cerebral cortex is the dentatothalamocortical (DTC) pathway. This tract is comprised of axons originating within the dentate nuclei that project through the ipsilateral SCP and, after decussation in the midbrain tegmentum, synapse within the contralateral ventrolateral nucleus of the thalamus. From the thalamus, second order axons terminate in the primary motor cortex as well as secondary and tertiary association areas within the frontal and parietal lobes. Thus, in addition to its influence on planned motor activity, the cerebellum via the DTC tract is believed to modulate cognition and behavior [158-160].

This non-traditional view of cerebellar function is supported by the recognition of cognitive, linguistic, and behavioral-affective impairments in children and adults with an injury or functional disturbance of the cerebellum and/or the proximal DTC pathway [99,100,161-164]. Cerebellar mutism syndrome (CMS) is an example of infratentorial injury leading to neurobehavioral dysfunction and is best described in children who have undergone surgical resection for an infratentorial tumor [99,165-167]. In medulloblastoma, the most common malignant brain tumor of childhood, CMS is estimated to occur in $25 \%$ of patients treated with surgery [166-168]. Mutism, oromotor/oculomotor apraxia, emotional lability, axial hypotonia, and cerebellar/brainstem dysfunction are the principal symptoms that typify this syndrome. Interestingly, symptom onset is usually $24-48$ hours after an otherwise unremarkable post-operative period. Treatment is primarily supportive, including, prolonged inpatient rehabilitation as well as gastrostomy and/or tracheostomy placement for severe cases. Recovery is variable. The most distressful symptoms- mutism and emotional lability- are generally transient, abating weeks to months after onset, but, significant long-term cognitive, behavioral, and/or motor deficits are frequently recognized in these children [169-172].

Clinical experience suggests the risk for developing CMS is increased in patients with midline embryonal tumors. Otherwise, there is no identified patient/tumor characteristic, surgical approach, or post-operative injury pattern that is universally accepted as a predictor of CMS $[161,165,173-175]$. The disrupted physiology underlying the striking neurobehavioral deficits is also largely unknown. Unfortunately, deficient understanding of CMS has limited the implementation of aggressive preventative and/or ameliorative measures. 
Perturbation of the cerebello-cerebral network is presumed to underlie many of the manifest symptoms of CMS $[100,176,177]$. Given its role as the primary outflow tract for the cerebellum and the vulnerability of its more proximal aspects to surgical injury, with respect to CMS, the DTC pathway has garnered increased attention $[165,167,168,175]$. To investigate the involvement of the DTC tracts in CMS, we reviewed conventional magnetic resonance imaging (MR) and diffusion tensor imaging (DTI) in a cohort of pediatric embryonal tumor patients. We hypothesized that preoperative tumor position within the $4^{\text {th }}$ ventricle and distance between the superior cerebellar peduncles (SCP) may predict the development of CMS, and, that anisotropy of water diffusion in proximal DTC tracts would be lower in those patients diagnosed with CMS.

\subsection{Subjects}

Between August 2003 and May 2006, 64 children were enrolled on an IRB approved institutional protocol designed for the treatment of children with newly diagnosed embryonal tumors. The majority of patients $(n=62)$ enrolled on this protocol had tumors located within the posterior fossa. After surgical resection, $21 \%$ of these patients (medulloblastoma $\mathrm{n}=10$ and atypical teratoid/rhabdoid tumor $\mathrm{n}=3$ ) were prospectively diagnosed with CMS based upon clinical symptoms and signs.

Retrospective review of post-operative clinical notes was often indeterminate with regard to CMS symptom onset, thus, diagnosis of CMS was arbitrarily reported as the primary surgical date. Of the 49 patients who did not develop CMS after posterior fossa tumor resection, 13 were randomly selected for comparative analyses.

\subsection{Diagnostic Imaging}

Magnetic resonance imaging was performed with a Siemens symphony $1.5 \mathrm{~T}$ or 3T scanner (Siemens Medical Solutions, Erlangen, Germany) using the standard quadrature headcoil. The imaging examination included standard T1-weighted, T2weighted, and FLAIR diagnostic scans and diffusion-weighted images for DTI. Diffusion weighted images were acquired with a single-shot, spin-echo, echo-planar acquisition sequence with eddy current balanced diffusion weighting gradient pulses to reduce distortion [178] ( 6 encoding directions with $\mathrm{b}=1000 \mathrm{sec} / \mathrm{mm}^{2}$; echo time $(\mathrm{TE}) /$ repetition time $(\mathrm{TR})=127 \mathrm{msec} / 10 \mathrm{sec}$; matrix $=128 \times 128$ on $230 \mathrm{~mm} \times 230 \mathrm{~mm}$ field of view (FOV); slices $3 \mathrm{~mm}$ without gap resulting in voxels of $1.8 \times 1.8 \times 3 \mathrm{~mm}$ ). Four measurements of the diffusion data were realigned and resliced (SPM2, [http://www.fil.ion.ucl.ac.uk/spm/]) before diffusion tensor processing and analysis. The diffusion tensor was solved using a weighted linear least-square fit implemented using the DTIfit algorithm as part of the Functional Magnetic Resonance Imaging of the Brain (FMRIB's) diffusion toolbox (FDT v2.0) (http://www.fmrib.ox.ac.uk/fsl/fdt/index.html). Voxel wise statistical analysis was then conducted using Tract Based Spatial Statistics (TBSS, v1.2). 


\subsection{Image Analysis}

Both pre-operative and post-operative conventional MR imaging were completed on all patients. Unfortunately, metal artifact and patient movement sufficiently obscured imaging in 3 patients (all with CMS). Thus, 23 patients (10 with CMS, 13 without CMS) comprised the final cohort. A single neuroradiologist (FHL) who was blinded to the diagnosis of CMS evaluated all conventional imaging. On the pre-operative imaging, tumor location was characterized as either midline or lateral (left vs. right), and the location/laterality of tumor invasion represented by abnormal T2 signal within the cerebral hemispheres, thalami, midbrain, pons, medulla, inferior/middle/superior cerebellar peduncles, and cerebellum were assessed. To characterize the relative cranial/caudal location of tumor, we measured on the sagittal T1-weighted image the extension of tumor above and below the line bisecting the $4^{\text {th }}$ ventricle and calculated the above/below ratio for each patient (Fig 7.1). To characterize preoperative stress on the $\mathrm{SCP}$, we measured on the T2-weighted image the distance (splay) between the superior cerebellar peduncles at the level of the rostral pons (Fig 7.1). A radiological diagnosis of hydrocephalus was noted and the degree of hydrocephalus was calculated using the Evan's Index. We hypothesized that the above/below ratio and SCP splay would be greater in patients with CMS because tumors residing higher in the posterior fossa would cause more preoperative stress on and require greater surgical manipulation of the cerebellar DTC outflow via the SCP. Two post-operative examinations, immediate and delayed, were reviewed. The degree of surgical resection as well as the location/laterality of abnormal T2 signal within similar head regions as the pre-operative scan was assessed. One patient (non-CMS) did not have an immediate post-operative film available for review $(\mathrm{N}=22)$. Fisher's exact test was used to compare proportions between groups (SAS v9.1 ${ }^{\circledR}$; SAS $^{\circledR}$ Institute, Cary, NC).

Diffusion tensor imaging preprocessing was performed using the Functional MRI of the Brain (FMRIB) Software Library (FSL) (FMRIB Centre, University of Oxford, and Oxford, United Kingdom). Fractional anisotropy (FA) images from the immediate post-operative scan were calculated from the diffusion tensor in each voxel. The tractbased spatial statistics (TBSS) tool in FSL was used to identify tract-based differences in FA values between the CMS and control patients. Tract-based spatial statistics computes a group mean FA skeleton, which represents the centers of all fiber bundles that are common to the subjects involved in the study [134]. The aligned FA image for each subject was projected onto the tract skeleton. The skeletonized FA maps for the groups were compared with the non-parametric permutation procedure called randomize in FSL. Differences in FA between the groups were considered statistically significant for $\mathrm{p} \leq$ 0.05 , after correction for multiple comparisons.

\subsection{Results}

All patients diagnosed with CMS were male in contrast to the control group which had 7 females (54\%) (Table 7.1). Otherwise, the 2 groups were similar with respect to age at diagnosis, treatment risk, extent of surgical resection, and timing of all 


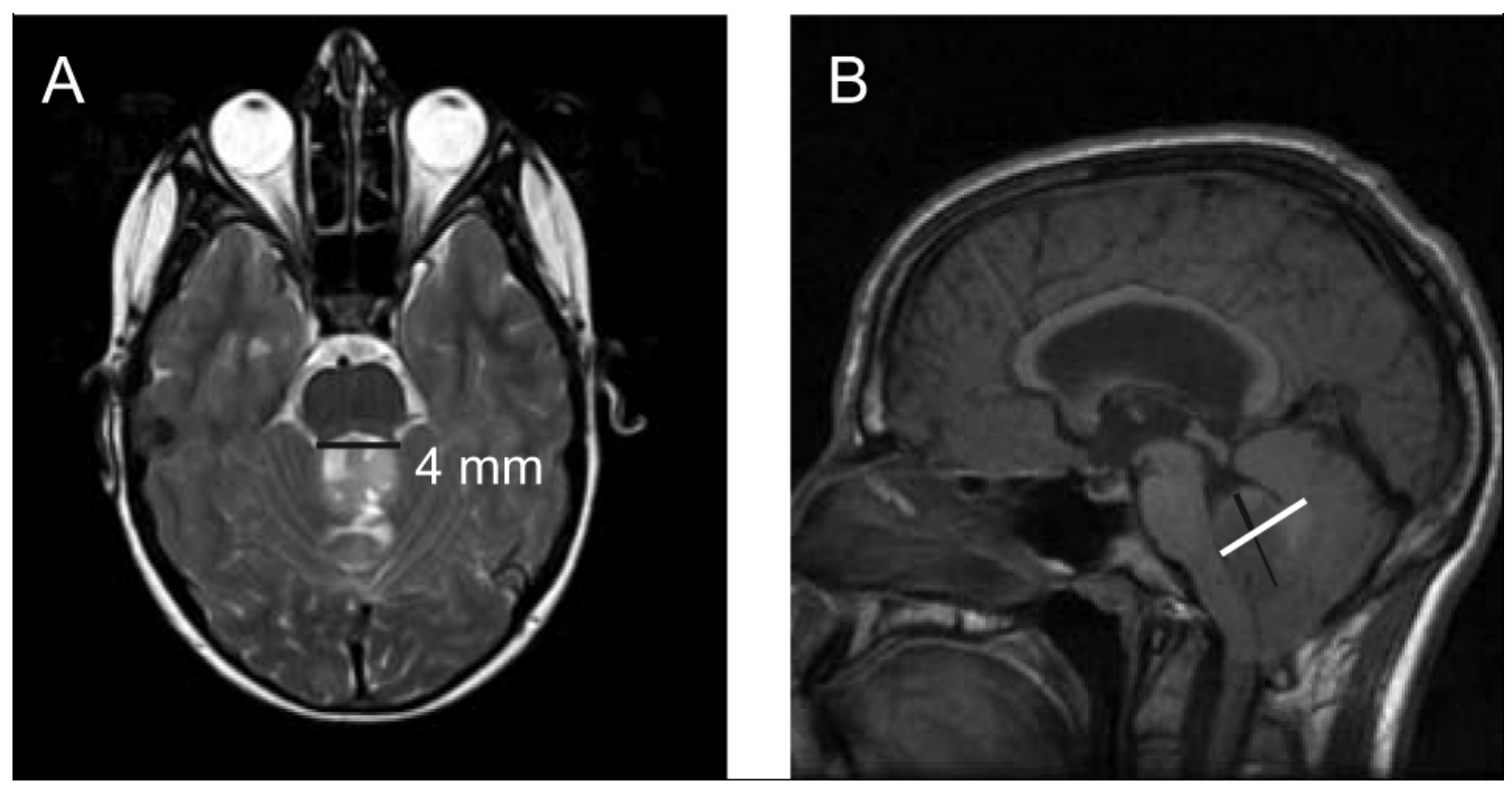

Figure 7.1 Depicts splay between SCP's (A) and above/below ratio (B) in a patient with medulloblastoma.

Table 7.1 Patient characteristics.

\begin{tabular}{lcc}
\hline Demographic Information & \multicolumn{2}{c}{ Cerebellar Mutism Syndrome } \\
\cline { 2 - 3 } & $\mathrm{No}$ & Yes \\
\hline $\begin{array}{l}\text { Gender } \\
\quad \text { Male }\end{array}$ & $\mathrm{N}=6(46 \%)$ & $\mathrm{N}=10(100 \%)$ \\
$\begin{array}{l}\text { Surgery Extent } \\
\quad \text { Gross total resection }\end{array}$ & $\mathrm{N}=10(77 \%)$ & $\mathrm{N}=9(90 \%)$ \\
$\quad \begin{array}{l}\text { Near total (90\%) } \\
\quad \text { Sub total (50-90\%) }\end{array}$ & $\mathrm{N}=1(8 \%)$ & $\mathrm{N}=0$ \\
$\begin{array}{l}\text { Age at Tumor Diagnosis (years; } \\
\text { median, range) }\end{array}$ & $7.8(3.7-20.2)$ & $\mathrm{N}=1(10 \%)$ \\
$\begin{array}{l}\text { Days from Surgery to Baseline Scan } \\
\text { Median (range) }\end{array}$ & $-2(-5,13)$ & $-2(-11,1)$ \\
$\begin{array}{l}\text { Days from Surgery to Post-Op Scan } \\
\text { Median (range) }\end{array}$ & $1.5(1,14)$ & $1(1,10)$ \\
$\begin{array}{l}\text { Days from Surgery to Follow-up Scan } \\
\text { Median (range) } \\
\text { Evan's Index of Hydrocephalus } \\
\text { Median (range) }\end{array}$ & $16(13,26)$ & $17(5,27)$ \\
\hline
\end{tabular}


scans. Consistent with the diagnosis of CMS, all patients exhibited abnormal speech and $8 / 10$ patients had documented irritability and/or agitation in the postoperative period. An increased above/below ratio that reflects a more rostral tumor position within the $4^{\text {th }}$ ventricle was statistically associated with the future development of CMS $(p=0.035)$. Otherwise, no statistical differences were detected at the baseline study between the two groups with regard to tumor invasion or in the splay between SCP $(p=0.11)$ (Table 7.2). The denate nuclei, superior cerebellar peduncle, and midbrain were rarely involved preoperatively. Majority of the patients (78\%) within the cohort had radiological evidence for hydrocephalus and the degree of pre-operative hydrocephalus was similar for both groups.

On the immediate post-operative scan following surgery, patients with CMS were more likely to have abnormal T2 signal involving the pons $(\mathrm{p}=0.029)$, midbrain $(\mathrm{p}=$ $0.003)$, and SCP $(p=0.030)$ than were those without CMS (Table 7.3). With respect to T2 signal abnormalities noted within the pons, dentate, SCP, and midbrain: most patients without CMS $(83 \%)$ had $\leq 1$ of these structures involved. However, the majority of patients with CMS (90\%) had abnormalities either in 3 or more of these structures (Table 7.3). Furthermore, bilateral injury within these structures was more common in patients with CMS than those patients without CMS (80\% vs. 15\%). On the delayed postoperative scan, no statistical differences between the 2 groups were observed. However, patients with CMS tended to have more abnormalities detected within the SCP $(p=0.10)$. Of note, because the dentate nuclei were involved frequently in both groups, no statistical differences were noted.

On the immediate post-operative scan, patients with CMS had significantly reduced FA in four areas: 1) bilateral SCP, 2) bilateral fornices (column), 3) white matter region proximate to the right angular gyrus (Tailerach coordinates $35-7119$ ), and 4) white matter region proximate to the superior frontal gyrus (Tailerach coordinates -2457 20) (Fig 7.2).

\subsection{Discussion}

Despite clinical heterogeneity, post-operative CMS in childhood brain tumor patients is one of the better described neuro-behavioral syndromes that can occur after infratentorial injury [99,165-167]. The diagnosis of CMS appears to be associated with worse neurological and cognitive outcomes [169-172]. One of the most intriguing aspects of this syndrome is that despite apparent pre-surgical similarities, only a subset of patients with posterior fossa tumors develops CMS after definitive resection. Surgical approach, tumor characteristics, and various perioperative factors (concomitant hydrocephalus, infection, and ischemia) have been posited as possible causative mechanisms [99,173,179-181]. However, none of these factors has been conclusively accepted. Preventative or ameliorative interventions are lacking, in part, because of this incomplete understanding. Given its projection to the cerebral cortex, proximal DTC involvement in CMS is presumed, but, this hypothesis has not been systematically examined [167,168,177,182,183]. 
Table 7.2 Frequency of $\mathrm{T} 2$ involvement in baseline imaging.

\begin{tabular}{|c|c|c|c|c|}
\hline \multirow[b]{2}{*}{ Variables } & \multicolumn{3}{|c|}{ Cerebellar Mustism Syndrome } & \multirow[b]{2}{*}{ P Value } \\
\hline & $\begin{array}{c}\text { Total } \\
(\mathrm{N}=23)\end{array}$ & $\begin{array}{c}\text { No } \\
(\mathrm{N}=13)\end{array}$ & $\begin{array}{c}\text { Yes } \\
(\mathrm{N}=\mathbf{1 0})\end{array}$ & \\
\hline Cerebellum & & & & $0.604^{\dagger}$ \\
\hline Bilateral (\%) & $1(100 \%)$ & $1(100.0 \%)$ & $0(0.0 \%)$ & \\
\hline None $(\%)$ & $18(100 \%)$ & $9(50.0 \%)$ & $9(50.0 \%)$ & \\
\hline Unilateral (\%) & $4(100 \%)$ & $3(75.0 \%)$ & $1(25.0 \%)$ & \\
\hline Dentate & & & & $0.104^{\dagger}$ \\
\hline None $(\%)$ & $19(100 \%)$ & $9(47.4 \%)$ & $10(52.6 \%)$ & \\
\hline Unilateral (\%) & $4(100 \%)$ & $4(100.0 \%)$ & $0(0.0 \%)$ & \\
\hline Inferior CP & & & & $0.656^{\dagger}$ \\
\hline Bilateral (\%) & $6(100 \%)$ & $3(50.0 \%)$ & $3(50.0 \%)$ & \\
\hline None $(\%)$ & $15(100 \%)$ & $8(53.3 \%)$ & $7(46.7 \%)$ & \\
\hline Unilateral (\%) & $2(100 \%)$ & $2(100.0 \%)$ & $0(0.0 \%)$ & \\
\hline Middle CP & & & & $0.360^{\dagger}$ \\
\hline None $(\%)$ & $17(100 \%)$ & $9(52.9 \%)$ & $8(47.1 \%)$ & \\
\hline Unilateral (\%) & $5(100 \%)$ & $4(80.0 \%)$ & $1(20.0 \%)$ & \\
\hline Superior CP & & & & $0.127^{\dagger}$ \\
\hline None $(\%)$ & $18(100 \%)$ & $12(66.7 \%)$ & $6(33.3 \%)$ & \\
\hline Unilateral (\%) & $5(100 \%)$ & $1(20.0 \%)$ & $4(80.0 \%)$ & \\
\hline Medulla & & & & $0.476^{\dagger}$ \\
\hline Bilateral (\%) & $3(100 \%)$ & $1(33.3 \%)$ & $2(66.7 \%)$ & \\
\hline None $(\%)$ & $18(100 \%)$ & $10(55.6 \%)$ & $8(44.4 \%)$ & \\
\hline Unilateral (\%) & $2(100 \%)$ & $2(100.0 \%)$ & $0(0.0 \%)$ & \\
\hline Pons & & & & $1.000^{\dagger}$ \\
\hline None $(\%)$ & $22(100 \%)$ & $12(54.5 \%)$ & $10(45.5 \%)$ & \\
\hline Unilateral (\%) & $1(100 \%)$ & $1(100.0 \%)$ & $0(0.0 \%)$ & \\
\hline Midbrain & & & & $1.000^{\dagger}$ \\
\hline None $(\%)$ & $21(100 \%)$ & $12(57.1 \%)$ & $9(42.9 \%)$ & \\
\hline Unilateral (\%) & $2(100 \%)$ & $1(50.0 \%)$ & $1(50.0 \%)$ & \\
\hline \multicolumn{5}{|l|}{ Thalamus } \\
\hline None $(\%)$ & $22(100 \%)$ & $13(59.1 \%)$ & $9(40.9 \%)$ & \\
\hline \multicolumn{5}{|c|}{ Cerebral Hemisphere } \\
\hline None (\%) & $22(100 \%)$ & $13(59.1 \%)$ & $9(40.9 \%)$ & \\
\hline
\end{tabular}


Table 7.3 Frequency of $\mathrm{T} 2$ involvement in immediate post-operative imaging.

\begin{tabular}{|c|c|c|c|c|}
\hline \multirow[b]{2}{*}{ Variables } & \multicolumn{3}{|c|}{ Cerebellar Mutism Syndrome } & \multirow[b]{2}{*}{ P Value } \\
\hline & $\begin{array}{c}\text { Total } \\
(\mathbf{N}=22)\end{array}$ & $\begin{array}{c}\text { No } \\
(\mathrm{N}=12)\end{array}$ & $\begin{array}{c}\text { Yes } \\
(N=10)\end{array}$ & \\
\hline Cerebellum & & & & $0.099^{\dagger}$ \\
\hline Bilateral (\%) & $13(100 \%)$ & $5(38.5 \%)$ & $8(61.5 \%)$ & \\
\hline Unilateral (\%) & $9(100 \%)$ & $7(77.8 \%)$ & $2(22.2 \%)$ & \\
\hline Dentate & & & & $0.646^{\dagger}$ \\
\hline Bilateral (\%) & $2(100 \%)$ & $1(50.0 \%)$ & $1(50.0 \%)$ & \\
\hline None $(\%)$ & $5(100 \%)$ & $4(80.0 \%)$ & $1(20.0 \%)$ & \\
\hline Unilateral (\%) & $15(100 \%)$ & $7(46.7 \%)$ & $8(53.3 \%)$ & \\
\hline Inferior CP & & & & $0.721^{\dagger}$ \\
\hline Bilateral (\%) & $13(100 \%)$ & $6(46.2 \%)$ & $7(53.8 \%)$ & \\
\hline None $(\%)$ & $5(100 \%)$ & $3(60.0 \%)$ & $2(40.0 \%)$ & \\
\hline Unilateral (\%) & $4(100 \%)$ & $3(75.0 \%)$ & $1(25.0 \%)$ & \\
\hline Middle CP & & & & $0.381^{\dagger}$ \\
\hline Bilateral (\%) & $4(100 \%)$ & $1(25.0 \%)$ & $3(75.0 \%)$ & \\
\hline None $(\%)$ & $7(100 \%)$ & $5(71.4 \%)$ & $2(28.6 \%)$ & \\
\hline Unilateral (\%) & $11(100 \%)$ & $6(54.5 \%)$ & $5(45.5 \%)$ & \\
\hline Superior CP & & & & $0.008^{\dagger}$ \\
\hline Bilateral (\%) & $1(100 \%)$ & $1(100.0 \%)$ & $0(0.0 \%)$ & \\
\hline None $(\%)$ & $11(100 \%)$ & $9(81.8 \%)$ & $2(18.2 \%)$ & \\
\hline Unilateral (\%) & $10(100 \%)$ & $2(20.0 \%)$ & $8(80.0 \%)$ & \\
\hline Medulla & & & & $0.308^{\dagger}$ \\
\hline
\end{tabular}




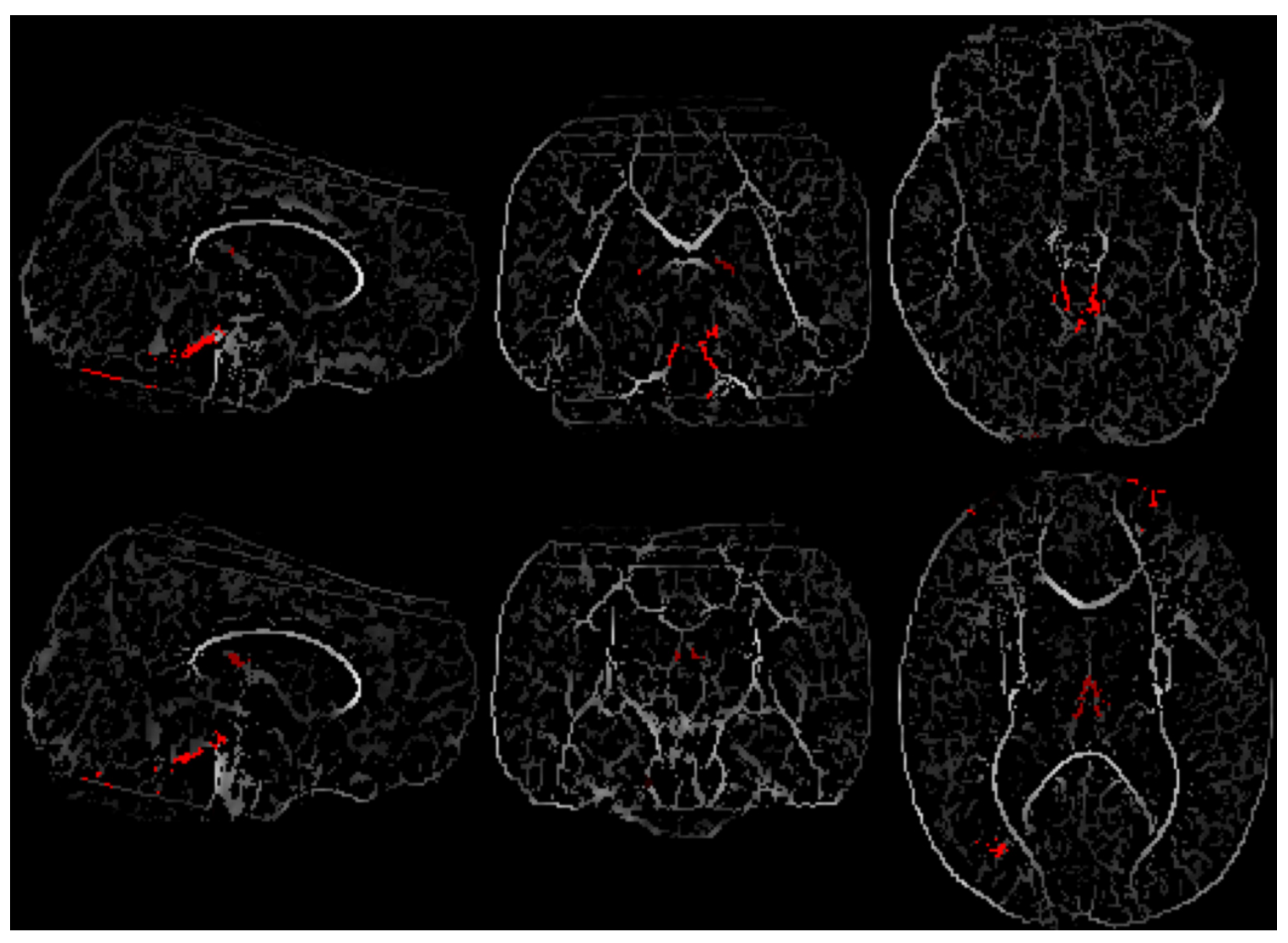

Figure 7.2 Post-operative scan of patients with CMS. These patients had significantly reduced FA in four areas: 1) bilateral SCP, 2) bilateral fornices (column), 3) white matter region proximate to the right angular gyrus (Tailerach coordinates $35-71$ 19), and 4) white matter region proximate to the superior frontal gyrus (Tailerach coordinates -2457 20). The tracts with reduced FA are shown as red overlay on the mean skeletonized FA map (some additional artifact can be seen at the edges of the images). 
This study represents the first blinded review of MR and diffusion weighted imaging obtained prospectively in a cohort of children after recent resection of a posterior fossa embryonal tumor. Comparing patients who did and did not develop CMS, perturbation of the proximal DTC was identified to be a potentially important determinant of this debilitating disorder. On immediate post-operative evaluation, conventional imaging showed evidence of proximal DTC involvement in those patients diagnosed with CMS. Previous studies have suggested that injury to the dentate nuclei is critical to the development of CMS [174,179]. Our findings are generally supportive; however, neither unilateral nor bilateral damage to the dentate was required for development of CMS. Rather, what distinguished patients with CMS was evidence for post-operative injury (increased T2 signal suggestive of edema) to the SCP and midbrain. Concurrent injury to the afferent corticopontine fibers may also be important as the pons was also more frequently involved in patients who developed CMS. When examining the putative posterior fossa anatomical structures (pons, dentate nuclei, SCP's, and midbrain), the majority of patients with CMS (90\%) were noted to have signal abnormalities within 3 or more of these structures. In contrast, none of the unaffected patients had $\geq 3$ structures involved. Furthermore, bilaterality may be an important associative finding as $80 \%$ of patients with CMS vs. $15 \%$ of patients without CMS had evidence of bilateral injury within these structures. Thus, "multiple, bilateral hits" within posterior fossa components of the cortico-cerebellar-cortical circuit may be required for CMS development. In a separate analysis utilizing the same cohort of patients, Miller, et al., observed a positive association between CMS and bilateral damage to the DTC tract as patients with bilateral damage were $\sim 12 \mathrm{x}$ more likely to develop CMS than those with unilateral injury only [184]. These findings are consistent with Crutchfield's early hypothesis that bilateral interruption of the DTC pathways may be responsible for the development of CMS [182].

To the best of our knowledge, the use of diffusion tensor imaging in CMS has not been previously reported. Consistent with our initial hypothesis, decreased integrity (as measured by decreased FA) among the white matter bundles comprising both SCP's was also evident in patients with CMS. Interestingly, whereas bilateral involvement of SCP is apparent on diffusion tensor imaging, on postoperative conventional imaging, unilateral signal abnormalities in these structures were typically observed. This discrepancy between conventional and diffusion tensor imaging suggests that a functional disturbance of the proximal efferent tract without a direct neuroanatomical correlate on conventional MR may be present. As supported by conventional imaging, the observed decreased anisotropy within the bilateral SCP's may not necessarily reflect direct but rather additive effects of remote injury (pons and/or dentate nuclei). Furthermore, as Pollock, et al., have previously suggested, the delayed onset of symptoms in some patients indicate that the involved structures in CMS are not likely directly damaged in surgery [99]. In the future, it would be interesting to correlate the timing of CMS onset with the pattern of injury to determine if patients with direct injury to bilateral SCP's exhibit symptoms sooner than patients with more remote albeit salient injury. Irrespective of timing and pattern of injury, our findings support that disruption of white matter tracts within the SCP's (either through direct injury or remote perturbation) may 
represent the underlying pathophysiological substrate for CMS in patients undergoing posterior fossa surgery.

Based on a hypothesized link between proximal DTC injury and CMS, we also evaluated two novel preoperative measures (tumor above/below ratio and SCP splay) that were designed to predict development of CMS. Indeed, patients with tumors that resided higher in the $4^{\text {th }}$ ventricle were statistically more likely to develop CMS. Tumor in this location may predispose CMS because there is more preoperative stress and increased vulnerability within the proximal DTC tract to operative injury. In this study, there was also a trend toward larger SCP splay in patients with CMS. In light of our findings noted above, a change in surgical approach based on these measures is not warranted.

However, increased post-operative surveillance of patients with tumors that reside high in the fourth ventricle and/or widen the splay of the SCP's should be given. A larger prospective study is required to determine the accuracy and thresholds of these measures for predicting CMS development.

Perhaps unexpectedly, diffusion tensor imaging also identified three supratentorial tracts where FA was lower in patients with CMS than in the control group. Detection of these areas highlights the value of TBSS for efficient and unbiased group comparison of diffusion tensor imaging data. Although speculative, the observed disordered water diffusion within the fornices and white matter tracts proximate to the right angular gyrus and left superior frontal gyrus, areas known to process spatial and temporal information essential for normal cognitive, linguistic, and motor behavior, is provocative and may represent or suggest specific cortical regions relevant to the neurobehavioral manifestations of CMS. Indeed, similar cortical findings using single photon emission tomography (SPECT) have been previously demonstrated. Marien, et $a l$, proposed a cerebello-cerebral diaschisis model to account for altered cortical function in a patient with aphasia following cerebellar ischemic infarct [185]. Later reports although not consistent have noted perfusion deficits measured by SPECT in children with CMS involving frontal as well as parieto-occipital areas that could be subserved by the white matter in which we observed low FA [186-188]. Thus our finding of disrupted cortically-based white matter tracts is consistent with the characterization of CMS as an example of the cerebellar cognitive affective syndrome proposed by Schmahmann [159]. Further elaboration of the neural substrates of CMS, including gray matter and white matter, may help to clarify the controversial role of the cerebellum in cognitve function [189].

The fornices are not a classical component of the DTC pathway. Indeed, decreased FA in these structures may be confounded by increased hydrocephalus in the CMS patients (Evans index $=0.43$ vs. 0.37 in non-CMS patients). Furthermore, to our knowledge anterograde amnesia has not been reported in patients with CMS (albeit, this would be very difficult to assess) as in other patients with bilateral fornical damage. However, several brainstem nuclei, most notably the ventral tegmental area within the midbrain, have reciprocal connections with limbic pathways that include the fornical columns [190]. Therefore, surgical or functional disruption of these nuclei with subsequent alteration of limbic homeostasis may underlie aspects of the behavioral- 
affective disorder commonly encountered in CMS. This suggested mechanism is speculative and needs to be further evaluated.

Several other limitations should be kept in mind when interpreting our data. First, despite well established cardinal signs and symptoms, CMS is a heterogeneous syndrome that has thus far defied a strict diagnostic criterion. Although our patients with CMS were identified prospectively, specific patient symptoms were not uniformly characterized. Thus, like other reports which characterize a CMS population, clinical heterogeneity within this cohort is expected and is not strictly accounted with respect to outcomes. Also, 3 patients had multiple surgeries prior to definitive resection; baseline scan was arbitrarily assigned as the initial surgical resection. Furthermore, because our institution serves as a national referral center, onset of CMS could not always be accurately determined as some patients were referred after primary surgery and onset of symptoms. Related to this issue, the pre-operative MR imaging were often completed at other centers and thus were not of uniform technique or quality. This heterogeneity could have affected the interpretation of our predictive measures. All patients with CMS in this study were male. We believe this to be a clinical anomaly rather that important pathological finding. Nevertheless, gender differences could influence our findings. Finally, the diffusion tensor imaging findings in the supratentorium should be interpreted cautiously. Decreased FA observed proximate to the right angular gyrus (Tailerach coordinates 35 -71 19) and superior frontal gyrus (Tailerach coordinates -24 57 20) could potentially be the result of susceptibility artifacts related to ventricular blood present after surgery, air/tissue interfaces within the frontal sinuses and/or external ear canal, as well as, metal devices (shunt, ommaya) secured on the surface of the skull.

Despite these limitations, our study adds significantly to the growing body of research that implicates the proximal DTC tracts in CMS. Using conventional MR and diffusion tensor imaging, our findings suggest multiple bilateral injuries to the proximal DTC pathways may predispose the development of CMS and that functional disruption of the white matter bundles containing efferent axons within the SCP's is a critical underlying pathophysiological component of CMS. When postoperative injury is remote from the SCP's, the cause or nature of this perturbation remains uncertain. With regard to clinical decision-making in patients with posterior fossa tumors, although measurements of splay and location were not robustly predictive, heightened concern should be given to those patients that harbor tumors high in the $4^{\text {th }}$ ventricle. Prospective studies, including preoperative diffusion tensor imaging, intra-operative or postoperative conventional, functional, and vascular imaging in conjunction with careful neurological and neurocognitive evaluations are needed to validate our findings and further clarify the neuroanatomical and functional substrates of this puzzling syndrome. 


\section{Chapter 8. Summary}

\subsection{Introduction}

In this dissertation, I presented a systemic body of research using Diffusion Tensor Imaging to evaluate, diagnose and treat pediatric brain tumor patients. I have discussed the theory and application of DTI as it was performed in a series of translational investigations at St. Jude Children's Research Hospital. I hypothesized that DTI would improve our ability to detect disease involvement in white matter pathways. I hypothesized that DTI would impact treatment which may alter the course of disease progression. And finally, I hypothesized that DTI would improve our understanding of how treatment affects cognitive and functional outcomes in pediatric brain tumor survivors.

It this chapter, I will outline the pertinent findings for each of the studies conducted for this dissertation in the following sections. I will review the objective of each study, discuss the study design, pertinent findings and finally offer a summary of the conclusions. I will then summarize all of these studies as they pertain to my stated hypotheses and specific aims.

\subsection{Use of DTI in Brainstem Gliomas}

Conventional magnetic resonance imaging (MRI) permits subcategorization of brainstem tumors by location and degree of focality; however assessment of white matter tract involvement by tumor is limited. Diffusion tensor imaging (DTI) is a promising method for visualizing white matter tract tumor involvement supratentorially. Here I investigated the ability of DTI to visualize and quantify white matter tract involvement in brainstem glioma (BSG) patients.

DTI data (whole head echo-planar, 1.5 Tesla) were retrospectively analyzed in 7 patients with BSG (6 diffuse, 1 focal), 4 patient controls, and 5 normal controls. Fractional anisotropy (FA) and apparent diffusion coefficient (ADC) were calculated from the diffusion tensor in 6 regions of interest: the bilateral corticospinal tracts, transverse pontine fibers, and medial lemnisci. Relations between FA and ADC values and results of the neurologic examinations were evaluated.

The corticospinal tracts and transverse pontine fibers were affected more often than the medial lemnisci. The DTI parameters (FA and ADC) were significantly altered in all tracts of patients with BSGs $(P<0.05)$, compared with those values in the control groups. There was potential clinical significance between the severity of cranial nerve deficit severity and decreased FA.

DTI provided superior visualization and quantification of tumor involvement in motor, sensory, and transverse pontine tracts, compared with that provided by 
conventional MRI. Thus, DTI may be a sensitive measure of tract invasion. Further prospective studies are warranted to assess the ability of DTI to delineate tumor focality and improve risk stratification in children with BSGs.

\subsection{Axonal Degeneration in Pediatric Brain Tumor Patients}

Diffusion tensor (DT) imaging has been used to predict postoperative motor function in patients with supra-tentorial tumors. I sought to determine whether DT imaging and white matter tractography could detect axonal degeneration in patients with brainstem tumors.

A cross-sectional, retrospective study of 7 patients with brainstem tumors and 8 healthy volunteers was performed. The DT imaging data were normalized and regions of interest (ROIs) with the highest probability of sensory and motor connections were selected using the Talairach Atlas to identify the 3D millimetric coordinates of white matter tracts. An iterative process involving fractional anisotropy (FA), apparent diffusion coefficients (ADCs), and color maps was developed to precisely select ROIs in the bilateral sensory and motor tracts. The FA and ADC values were calculated for each ROI.

The FA values of sensory and motor tracts significantly differed between the patient and healthy volunteer groups ( $p<002, C>0.05$ ), whereas no significant changes were found in the splenium or genu of the corpus callosum. The FA values were altered proximal and distal to the brainstem tumors with a bimodal peak of antegrade decreased FA involving second- and third-order sensory axons and retrograde decreased FA of motor axons.

This study demonstrates changes in diffusion properties of sensory and motor tracts consistent with degeneration to further characterize brainstem tumors in children, and the results warrant the planning of prospective trials. The rigorous methods I describe may provide valuable information when planning biopsies or debulking of unusual brainstem tumors, as well as improve prognostication of the possible functional tract recovery following therapy.

\subsection{Utility of DTI in Surgical Planning}

Brainstem gliomas are a heterogeneous group of lesions that account for $15 \%$ of all pediatric tumors of the central nervous system. Diagnosis and treatment planning for these tumors is based on the observation of Epstein and Farmer that the growth of lesions with low malignant potential is limited by the anatomical structures of the brainstem.

Surgery is offered only to those patients with a high probability of harboring a low-grade tumor, because the attendant risk for significant morbidity outweighs the therapeutic benefit of debulking the tumor in cases of high-grade tumors. 
I reported two cases that highlight the potential of diffusion tensor (DT) imaging to identify local white matter tracts in the pons, medulla, and cervical cord and to improve the preoperative assessment of low-grade gliomas. Preoperative DT imaging in both cases demonstrated that the white matter tracts were displaced by the bulk of the low-grade tumors but were structurally preserved. Intraoperative and neurological findings were consistent with the pre-operative interpretation of the DT images. These cases demonstrate that DT imaging is a useful method for visualizing the relationship between tumor and normal brainstem white matter architecture, as well as for improving the surgical evaluation and management of pediatric brainstem tumors.

\subsection{Evaluation of Medulloblastoma Therapy with DTI}

Medulloblastoma is a subclass of Primitive Neuroectodermal Tumors (PNET) that arises exclusively in the cerebellum. It is the most frequent malignant brain tumor in children and is uniformly fatal in untreated patients. With contemporary treatment and the absence of metastasis at diagnosis, 5 year survival can be as high as 80 percent with at least 50 percent of children surviving until adulthood. Unfortunately, the majority of these survivors will face life long learning problems and diminished IQ levels. A body of evidence suggests that disturbances in attention, memory and reading may be related to a radiation insult to otherwise healthy nervous tissue.

I hypothesized that behavioral deficits in reading decoding among medulloblastoma survivors reflect therapy-induced disruption of the maturation of ventral visual processing during the development of reading skills. Functional imaging studies indicate that medulloblastoma survivors have delayed development of left lateralized neural networks that are associated with normal reading maturation. The decreased laterality of the extrastriate visual pathway in patients when compared to age related siblings, may involve disruption of the transcommisural white matter pathways.

Nine and 12 month interval patients demonstrated significantly reduced FA $(\mathrm{p}<0.05)$ in four areas: 1) Bilateral pulvinar, 2) Gyrus cinguli, 3) Bilateral corona radiata, and 4) Bilateral corticospinal tracts. Additionally, baseline vs. 12 month image patients also displayed significantly improved FA $(p<0.05)$ in three areas: 1$)$ The splenium of the corpus callosum, 2) The genu of the corpus callosum, and 3) Right middle temporal lobe. The reduced FA seen in the corticospinal tracts and corona radiata findings mimic those

discussed in Chapter 4 were we demonstrated the loss of anisotropy in distal white matter pathways correlated with focal insults in brainstem structures. As such, these changes in FA most likely represents the evolution of axonal degeneration changes in the distal motor and sensory pathways of brain stem structures that have received full dose radiation. Anterior cingulate gyrus changes are most likely represent changes in FA seen in the corticospinal and corona radiata, due to the presence of large pyramidal neurons in the anterior cingulate gyrus that play an important part in executing voluntary motor and premotor controls. 
Anatomical studies have demonstrated that the pulvinar pulvinar plays a key role in directing visual information and theoretically plays a role in visual attention. Given this evidence I strongly suspect that the significant decrease in FA in the pulvinar seen in the medulloblastoma patients at one year represents evidence for treatment induced neurotoxicity associated with visuospatial attention deficits. Kastner and Ungerleider (2000), demonstrated that the pulvinar is part of a network controlling visual spatial attention. Karnath, et al. (2002), and demonstrated that patients with lesions in the pulvinar exhibited visual spatial neglect. This supports the theory that disruption of the pulvinar would induce cognitive deficits in visual spatial attention and possibly impact reading.

\subsection{DTI in Cerebellar Mutism}

Cerebellar mutism syndrome (CMS) is a post-operative complication characterized by cerebellar dysfunction, oromotor/oculomotor apraxia, emotional lability, and mutism. The underlying neuroanatomical substrates of CMS are unknown, but dentatothalamocortical (DTC) tracts have been implicated. I used pre- and postoperative neuroimaging to investigate proximal DTC involvement in childhood embryonal brain tumor patients who developed CMS following tumor resection.

Diagnostic imaging from a cohort of 26 children previously operated for an embryonal brain tumor (13 patients prospectively diagnosed with CMS and 13 nonaffected patients) were evaluated. Pre-operative magnetic resonance (MR) imaging was used to define relevant tumor features, including two potentially predictive measures. Post-operative MR and diffusion tensor imaging were used to characterize operative injury and tract-based differences in anisoptropy of water diffusion.

In patients who developed CMS, initial tumor resided higher in the $4^{\text {th }}$ ventricle ( $p$ $=0.035$ ). Post-operative MR signal abnormalities within the SCP's and midbrain were observed more often in patients with CMS ( $p=0.030$ and 0.003 , respectively). The fractional anisotropy (FA) of water was lower in the bilateral SCP's, in the bilateral fornices, and two cortical areas.

My findings suggest multiple bilateral injuries to the proximal DTC pathways may predispose the development of CMS and that functional disruption of the white matter bundles containing efferent axons within the SCP's is a critical underlying pathophysiological component of CMS. Decreased FA in the fornices and cerebral cortex may be related to the abnormal neurobehavioral symptoms of CMS.

\subsection{Conclusions}

This research has generated important insights into the fundamental causes of neuroanatomical and cognitive deficits associated with cancer and cancer therapy. The use of DTI has permitted me to identify potential targets for improved radiological and 
surgical techniques as well as targets for pharmacological and behavioral interventions that might improve cognitive function in cancer survivors. The discoveries here will afford an opportunity to reduce the negative effects of cancer therapy on patients treated in the future while maintaining successful survival rates.

In Chapter 3 I presented evidence of how the introduction of this technique to pediatric brain stem tumor patients improved visualization of white matter tract invasion and could improve diagnosis and treatment by discriminating focal from diffuse brain stem gliomas. In my study on axonal degeneration I demonstrated that DTI was a useful adjunct to treatment planning and therapy. By demonstrating distant changes in FA correlated to axonal degeneration we consider DTI to be a useful tool in predicting recovery of neurological function presurgically. In Chapter 5, I further demonstrated the utility of DTI in the use of presurgical planning by highlighting two important cases in which both the diagnosis and surgical treatment were directly affected by the DTI studies. Next I implemented my DTI technique to assess post treatment sequela in medulloblastoma survivors and demonstrated DTI's usefulness in identify white matter changes important to cognition and reading. I also emphasized how this information could used to impact radiation therapy planning with the goal in mind to reduce cognitive sequela while preserving overall survival rates. And finally, I demonstrated how DTI was used to investigate the post surgical cognitive morbidity called cerebellar mutism and how this technique demonstrated post surgical changes in directly involved and indirectly involved white matter pathways. I believe that these findings improve the overall understanding of the underlying pathopysiology of the CMS and in time allow for the improvement of surgical outcomes.

Overall, I believe I have demonstrated that DTI can a benefit in the detection and treatment of children with brain tumors. I believe that the employment of DTI as outlined in this series of studies has made a difference in the treatment outcomes of patients. Additionally, the information gained from these studies will lead to new avenues of discovery and innovation that will not only benefit pediatric brain tumor patients, but may ultimately impact children with a wide variety of neurocognitive deficits, such as reading dyslexia and autism. 


\section{List of References}

1. Vlaardingerbroek MT, den Boer JA. Magnetic resonance imaging: Theory and practice, 2nd ed. Heidelberg: Springer, 1999.

2. Poustchi-Amin M, Mirowitz SA, Brown JJ, McKinstry RC, Li T. Principles and applications of echo-planar imaging: A review for the general radiologist. Radiographics 2001;21:767-779.

3. Guye M, Parker GJM, Symms M, et al. Combined functional MRI and tractography to demonstrate the connectivity of the human primary motor cortex in vivo. Neuroimage 2003;19:1349-1360.

4. Rye DB. Tracking neural pathways with MRI. Trends in Neurosciences 1999;22:373-374.

5. Hutchins B, Weber J. A rapid myelin stain for frozen section: Modifications of the Heidenhain procedure. Journal of Neuroscience Methods 1983;7:289-294.

6. Bernstein MA, King KF, Zhou XJ. Handbook of MRI pulse sequences. Ansterdam: Elsevier, 2004.

7. Haacke EM, Brown RW, Thompson MR, Venkatesan R. Magnetic resonance imaging: Physical principles and sequence design. New York: Wiley-Liss, 1999.

8. Le Bihan D, Mangin JF, Poupon C, et al. Diffusion tensor imaging: Concepts and applications. Journal of Magnetic Resonance Imaging 2001;13:534-546.

9. Stejskal EO, Tanner JE. Spin diffusion measurements: Spin echoes in presence of a time-dependent field gradient. Journal of Chemical Physics 1965;42:288.

10. Kingsley PB. Introduction to diffusion tensor imaging mathematics: Part III. Tensor calculation, noise, simulations, and optimization. Concepts in Magnetic Resonance Part A 2006;28A:155-179.

11. Kingsley PB. Introduction to diffusion tensor imaging mathematics: Part II. Anisotropy, diffusion-weighting factors, and gradient encoding schemes. Concepts in Magnetic Resonance Part A 2006;28A:123-154.

12. Heinbockel J. Introduction to tensor calculus and continuum mechanics. Canada: Trafford Publishing, 1996:181.

13. Mukherjee P, Miller JH, Shimony JS, et al. Diffusion-tensor MR imaging of gray and white matter development during normal human brain maturation. American Journal of Neuroradiology 2002;23:1445-1456. 
14. Hasan KM, Kanabar BP, Santos RM, Ewing-Cobbs L, Narayana PA. Age dependence of the fractional anisotropy of genu and splenium of human corpus callosum using optimized DT-MRI. Proceedings of the International Society of Magnetic Resonance in Medicine 2004;12:338.

15. Norris DG. The effects of microscopic tissue parameters on the diffusion weighted magnetic resonance imaging experiment. Nuclear Magnetic Resonance in Biomedicine 2001;14:77-93.

16. Szafer A, Zhong J, Gore JC. Theoretical model for water diffusion in tissues. Magnetic Resonance in Medicine 1995;33:697-712.

17. Mac Donald CL, Dikranian K, Song SK, Bayly PV, Holtzman DM, Brody DL. Detection of traumatic axonal injury with diffusion tensor imaging in a mouse model of traumatic brain injury. Experimental Neurology 2007;205:116-131.

18. Mac Donald CL, Dikranian K, Bayly P, Holtzman D, Brody D. Diffusion tensor imaging reliably detects experimental traumatic axonal injury and indicates approximate time of injury. Journal of Neuroscience 2007;27:11869-11876.

19. Klingberg T, Hedehus M, Temple E, et al. Microstructure of temporo-parietal white matter as a basis for reading ability: Evidence from diffusion tensor magnetic resonance imaging. Neuron 2000;25:493-500.

20. Schmithorst VJ, Wilke M, Dardzinski BJ, Holland SK. Cognitive functions correlate with white matter architecture in a normal pediatric population: A diffusion tensor MRI study. Human Brain Mapping 2005;26:139-147.

21. Wilke M, Sohn JH, Byars AW, Holland SK. Bright spots: Correlations of gray matter volume with IQ in a normal pediatric population. Neuroimage 2003;20:202-215.

22. Kingsley PB. Introduction to diffusion tensor imaging mathematics: Part III. Tensor calculation, noise, simulations, and optimization. Concepts in Magnetic Resonance Part A 2006;28A:155-179.

23. Miller AJ, Joseph PM. The use of power images to perform quantitative-analysis on low SNR MR-images. Magnetic Resonance Imaging 1993;11:1051-1056.

24. Dietrich O, Herlihy A, Dannels WR, et al. Diffusion-weighted imaging of the spine using radial k-space trajectories. Magnetic Resonance Materials in Physics Biology and Medicine 2001;12:23-31.

25. Rueckert D, Sonoda LI, Hayes C, Hill DLG, Leach MO, Hawkes DJ. Nonrigid registration using free-form deformations: Application to breast MR images. IEEE Transactions on Medical Imaging 1999;18:712-721. 
26. Jin J, Wang Q, Shen Y. Global optimization of medical image registration based on nonlinear correlation measurement. Conference Proceedings of the IEEE Enginering in Medicine and Biology Society 2005;4:3390-3393.

27. Smith SM, Jenkinson M, Johansen-Berg H, et al. Tract-based spatial statistics: Voxelwise analysis of multi-ssubject diffusion data. Neuroimage 2006;31:14871505.

28. Smith SM, Johansen-Berg H, Jenkinson M, et al. Acquisition and voxelwise analysis of multi-subject diffusion data with Tract-Based Spatial Statistics. Nature Protocols 2007;2:499-503.

29. Barkovich AJ. Concepts of myelin and myelination in neuroradiology. American Journal of Neuroradiology 2000;21:1099-1109.

30. Lesniak MS, Klem JM, Weingart J, et al. Brain stem gliomas: A classic system based on magnetic resonance imaging. Pediatric Neurosurgery 1991;16:73-83.

31. Barkovich A. Pediatric neuroimaging, 3rd ed. Philadelphia: Lippincott, Williams and Wilkins, 2003.

32. Gauvain KM, McKinstry RC, Mukherjee $\mathrm{P}$, et al. Evaluating pediatric brain tumor cellularity with diffusion-tensor imaging. American Journal of Roentgenology 2001;177:449-454.

33. Tien RD, Felsberg GJ, Friedman H, Brown M, MacFall J. MR imaging of highgrade cerebral glioms: Value of diffusion-weighted echoplanar pulse sequences. American Journal of Roentgenology 1994;671-677.

34. Guo AC, Cummings TJ, Dash RC, Provenzale JM. Lymphomas and high-grade astrocytomas: Comparison of water diffusibility and histologic characteristics. Radiology 2002;224:177-183.

35. Sinha S, Bastin ME, Whittle IR, Wardlaw JM. Diffusion tensor MR imaging of high-grade cerebral gliomas. American Journal of Neuroradiology 2002;23:520527.

36. Witwer BP, Moftakhar R, Hasan KM, et al. Diffusion-tensor imaging of white matter tracts in patients with cerebral neoplasm. Journal of Neurosurgery 2002;97:568-575.

37. Clark CA, Barrick TR, Murphy MM, Bell BA. White matter fiber tracking in patients with space-occupying lesions of the brain: A new technique for neurosurgical planning? Neuroimage 2003;20:1601-1608.

38. Field AS, Alexander AL, Wu YC, Hasan KM, Witwer B, Badie B. Diffusion tensor eigenvector directional color imaging patterns in the evaluation of cerebral 
white matter tracts altered by tumor. Journal of Magnetic Resonance Imaging 2004;20:555-562.

39. Valdes-Gorcia J, Espinoza-Diaz DM, Paredes-Diaz E. Stereotactic biopsy of brain stem and posterior fossa lesions in children. Acta Neurochirurgica (Wien ) 1998;140:899-903.

40. Reese TG, Heid O, Weisskoff RM, Wedeen VJ. Reduction of eddy-currentinduced distortion in diffusion MRI using a twice-refocused spin echo. Magnetic Resonance in Medicine 2003;49:177-182.

41. Poldrack R. Diffusion tensor imaging toolbox for SPM 99. http://sourceforge.net/projects/spm-toolbox/ Accessed March 1st 2003.

42. Pajevic S, Pierpaoli C. Color schemes to represent the orientation of anisotropic tissues from diffusion tensor data: Application to white matter fiber tract mapping in the human brain. Magnetic Resonance in Medicine 1999;42:526-540.

43. Allen J. Neurology and neurosurgery, 4th ed. Amsterdam, New York: Excerpta Medica Foundation, 1983.

44. Albright TD, Jessell TM, Kandel ER, Posner MI. Neural science: A century of progress and the mysteries that remain. Cell 2000;100 Suppl:S1-55.

45. Jallo GI, Freed D, Roonprapunt C, Epstein F. Current management of brainstem gliomas. Annals of Neurosurgery 2003;3:1-17.

46. Choux M, Lena G, Do L. Brainstem tumors, 1st ed. New York: Churchill Livingstone, 2000:471-491.

47. Albright AL, Packer RJ, Zimmerman R, et al. Magnetic-resonance scans should replace biopsies for the diagnosis of diffuse brain-stem gliomas: A report from the Childrens Cancer Group. Neurosurgery 1993;33:1026-1030.

48. Fiscbein NJ, Prados MD, Wara W, Russo C, Edwards MS, Barkovish AJ. Radiological classification of brain stem tumors: Correlation of magnetic resonance imaging appearance with clinical outcome. Pediatric Neurosurgery 1996;9-23.

49. Lesniak MS, Klem JM, Weingart J, Carson BS. Surgical outcomes following resection of contrast-enhanced pediatric brainstem gliomas. Pediatric Neurosurgery 2003;39:314-322.

50. Stieltjes B, Kaufmann WE, van Zijl PCM, et al. Diffusion tensor imaging and axonal tracking in the human brainstem. Neuroimage 2001;14:723-735. 
51. Pierpaoli C, Barnett A, Pajevic S, et al. Water diffusion changes in Wallerian degeneration and their dependence on white matter architecture. Neuroimage 2001;13:1174-1185.

52. Basser PJ, Pajevic S, Pierpaoli C, Duda J, Aldroubi A. In vivo fiber tractography using DT-MRI data. Magnetic Resonance in Medicine 2000;44:625-632.

53. Conturo TE, Lori NF, Cull TS, et al. Tracking neuronal fiber pathways in the living human brain. Proceedings of the National Academy of Sciences of the United States of America 1999;96:10422-10427.

54. Khong PL, Zhou LJ, Ooi GC, Chung BHY, Cheung RTF, Wong VCN. The evaluation of Wallerian degeneration in chronic paediatric middle cerebral artery infarction using diffusion tensor MR imaging. Cerebrovascular Diseases 2004;18:240-247.

55. Kuhn MJ, Mikulis DJ, Ayoub DM, Kosofsky BE, Davis KR, Taveras JM. Wallerian degeneration after cerebral infarction: Evaluation with sequential MRimaging. Radiology 1989;172:179-182.

56. Thomalla G, Glauche V, Koch MA, Beaulieu C, Weiller C, Rother J. Diffusion tensor imaging detects early Wallerian degeneration of the pyramidal tract after ischemic stroke. Neuroimage 2004;22:1767-1774.

57. Werring DJ, Toosy AT, Clark CA, et al. Diffusion tensor imaging can detect and quantify corticospinal tract degeneration after stroke. Journal of Neurology, Neurosurgery and Psychiatry 2000;69:269-272.

58. Beppu T, Inoue T, Kuzu Y, Ogasawara K, Ogawa A, Sasaki M. Utility of threedimensional anisotropy contrast magnetic resonance axonography for determining condition of the pyramidal tract in glioblastoma patients with hemiparesis. Journal of Neuro-Oncology 2005;73:137-144.

59. Taoka T, Sakamoto M, Iwasaki S, et al. Diffusion tensor imaging in cases with visual field defect after anterior temporal lobectomy. American Journal of Neuroradiology 2005;26:797-803.

60. Kuhn MJ, Johnson KA, Davis KR. Wallerian degeneration: Evaluation with MR imaging. Radiology 1988;168:199-202.

61. Waller AV. Experiments on the section of the glossopharyngeal and hypoglossal nerves of the frog, and observations of the alterations produced thereby in the structure of their primitive fibers. Philosophical Transactions fo the Royal Society London B, Biological Science 1850;140:423-429.

62. Kuhn MJ, Mikulis DJ, Ayoub DM, Kosofsky BE, Davis KR, Taveras JM. Wallerian degeneration after cerebral infarction: Evaluation with sequential MR imaging. Radiology 1989;172:179-182. 
63. Pennock JM, Rutherford MA, Cowan FM, Bydder GM. MRI: Early-onset of changes in wallerian degeneration. Clinical Radiology 1993;47:311-314.

64. Fellgiebel A, Wille P, Muller MJ, et al. Ultrastructural hippocampal and white matter alterations in mild cognitive impairment: A diffusion tensor imaging study. Dementia and Geriatric Cognitive Disorders 2004;18:101-108.

65. Khong PL, Zhou LJ, Ooi GC, Chung BHY, Cheung RTF, Wong VCN. The evaluation of Wallerian degeneration in chronic paediatric middle cerebral artery infarction using diffusion tensor MR imaging. Cerebrovascular Diseases 2004; 18:240-247.

66. Thomalla G, Glauche V, Koch MA, Beaulieu C, Weiller C, Rother J. Diffusion tensor imaging detects early Wallerian degeneration of the pyramidal tract after ischemic stroke. Neuroimage 2004;22:1767-1774.

67. Laundre BJ, Jellison BJ, Badie B, Alexander AL, Field AS. Diffusion tensor imaging of the corticospinal tract before and after mass resection as correlated with clinical motor findings: Preliminary data. American Journal of Neuroradiology 2005;26:791-796.

68. Helton KJ, Phillips NS, Khan RB, et al. Diffusion tensor imaging of tract involvement in children with pontine tumors. American Journal of Neuroradiology 2006;27:786-793.

69. Friston K, Ashburner J, Poline J, Frith CD, Heather JD, Frackowiak RSJ. Spatial registration and normalization of images. Human Brain Mapping 1995;2:165-189.

70. Brett M, Leff AP, Rorden C, Ashburner J. Spatial normalization of brain images with focal lesions using cost function masking. Neuroimage 2001;14:486-500.

71. Tien RD, Felsberg GJ, Friedman H, Brown M, MacFall J. MR imaging of highgrade cerebral glioms: Value of diffusion-weighted echoplanar pulse sequences. American Journal of Roentgenology 1994;671-677.

72. Hagmann P, Thiran JP, Jonasson L, et al. DTI mapping of human brain connectivity: Statistical fibre tracking and virtual dissection. Neuroimage 2003;19:545-554.

73. Kuhn MJ, Johnson KA, Davis KR. Wallerian degeneration: Evaluation with MR imaging. Radiology 1988;168:199-202.

74. Werring DJ, Toosy AT, Clark CA, et al. Diffusion tensor imaging can detect and quantify corticospinal tract degeneration after stroke. Journal of Neurology Neurosurgery and Psychiatry 2000;69:269-272. 
75. Beaulieu C, Does MD, Snyder RE, Allen PS. Changes in water diffusion due to Wallerian degeneration in peripheral nerve. Magnetic Resonance in Medicine 1996;36:627-631.

76. Castillo M, Smith JK, Kwock L, Wilber K. Apparent diffusion coefficients in the evaluation of high-grade cerebral gliomas. American Journal of Neuroradiology 2001;22:60-64.

77. Holodny A, Ollenschlager M, Melhem, ER. Diffusion imaging in brain tumors. Neuroimaging Clinics of North America 2002;12:107-124.

78. Kingsley RE. Concise text of neuroscience. Philadelphia: Lippincott, Williams \& Wilkins, 2000.

79. Block F, Dihne M, Loos M. Inflammation in areas of remote changes following focal brain lesion. Progress in Neurobiology 2005;75:342-365.

80. Donaldson SS, Laningham F, Fisher PG. Advances toward an understanding of brainstem gliomas. Journal of Clinical Oncology 2006;24:1266-1272.

81. Sandri A, Sardi N, Genitori L, et al. Diffuse and focal brain stem tumors in childhood: Prognostic factors and surgical outcome. Childs Nervous System 2006;22:1127-1135.

82. Gilbertson RJ, Hill DA, Hernan R, et al. ERBB1 is amplified and overexpressed in high-grade diffusely infiltrative pediatric brain stem glioma. Clinical Cancer Research 2003;9:3620-3624.

83. Roujeau T, Machado G, Garnett MR, et al. Stereotactic biopsy of diffuse pontine lesions in children. Journal of Neurosurgery 2007;107:1-4.

84. Phillips NS, Sanford RA, Helton KJ, et al. Diffusion tensor imaging of intraaxial tumors at the cervicomedullary and pontomedullary junctions: Report of two cases. Journal of Neurosurgery 2005;103:557-562.

85. Epstein F, Wisoff J. Intra-axial tumors of the cervicomedullary junction. Journal of Neurosurgery 1987;67:483-487.

86. Robertson PL, Allen JC, Abbott IR, Miller DC, Fidel J, Epstein FJ.

Cervicomedullary tumors in children: A distinct subset of brain-stem gliomas. Neurology 1994;44:1798-1803.

87. Weiner HL, Freed D, Woo HH, Rezai AR, Kim R, Epstein FJ. Intra-axial tumors of the cervicomedullary junction: Surgical results and long-term outcome.

Pediatric Neurosurgery 1997;27:12-18.

88. Epstein F, Farmer J. Brain-stem glioma growth patterns. Journal of Neurosurgery 1993;78:408-412. 
89. Scherer H. Structural development in gliomas. The American Journal of Cancer 1938;34:333-351.

90. Jellison BJ, Field AS, Medow J, Lazar M, Salamat MS, Alexander AL. Diffusion tensor imaging of cerebral white matter: A pictorial review of physics, fiber tract anatomy, and tumor imaging patterns. American Journal of Neuroradiology 2004;356-369.

91. Mori S, Frederiksen K, van Zijl PCM, et al. Brain white matter anatomy of tumor patients evaluated with diffusion tensor imaging. Annals of Neurology 2002;51:377-380.

92. Wheeler-Kingshott CAM, Hickman SJ, Parker GJM, et al. Investigating cervical spinal cord structure using axial diffusion tensor imaging. Neuroimage 2002;16:93-102.

93. Reese TG, Heid O, Weisskoff RM, Wedeen VJ. Reduction of eddy-currentinduced distortion in diffusion MRI using a twice-refocused spin echo. Magnetic Resonance in Medicine 2003;49:177-182.

94. Holder CA, Muthupillai R, Mukundan S, Eastwood JD, Hudgins PA. Diffusionweighted MR imaging of the normal human spinal cord in vivo. American Journal of Neuroradiology 2000;21:1799-1806.

95. Lenhard RE, Osteen RT, Gansler T. The American Cancer Society's Clinical Oncology, 1st ed. Atlanta: American Cancer Society, 2001.

96. Packer RJ, Goldwein J, Nicholson HS, et al. Treatment of children with medulloblastomas with reduced-dose craniospinal radiation therapy and adjuvant chemotherapy: A children's cancer group report. Journal of Clinical Oncology 1999; 17:2127-2136.

97. The Merck Manual, 17th ed. West Point PA: Merck and Co., 1999.

98. Madden DJ, Whiting WL, Huettel SA, White LE, MacFall JR, Provenzale JM. Diffusion tensor imaging of adult age differences in cerebral white matter: Relation to response time. Neuroimage 2004;21:1174-1181.

99. Pollack IF, Polinko P, Albright AL, Towbin R, Fitz C. Mutism and pseudobulbar symptoms after resection of posterior-fossa tumors in children: Incidence and pathophysiology. Neurosurgery 1995;37:885-893.

100. Schmahmann JD, Sherman JC. The cerebellar cognitive affective syndrome. Brain 1998; 121:561-579.

101. Zou P, Mulhern RK, Butler RW, Li C-S, Langston JW, Ogg RJ. BOLD responses to visual stimulation in survivors of childhood cancer. Neuroimage 2005;24:6169. 
102. Conners CK. Conners' Continuous Performance Test. Toronto: Multi-Health Systems Inc., 1995.

103. Riccio CA, Reynolds CR. Continuous performance tests are sensitive to ADHD in adults but lack specificity. A review and critique for differential diagnosis. Annals of the New York Acadamy of Science 2001;931:113-139.

104. Gajjar A, Chintagumpala M, Ashley D. Risk-adapted craniospinal radiotherapy followed by high-dose chemotherapy and stem-cell rescue in children with newly diagnosed medulloblastoma (St Jude Medulloblostoma-96): Long-term results from a prospective, multicentre trial (vol 7, pg 813, 2006). Lancet Oncology 2006;7:797.

105. Fiez JA, Petersen SE. Neuroimaging studies of word reading. Proceedings of the National Academy of Sciences of the United States of America 1998;95:914-921.

106. Joseph R. The limbic language/language axis theory of speech. Behavioral and Brain Sciences 2000;23:439-441.

107. Price CJ, Wise RJS, Frackowiak RSJ. Demonstrating the implicit processing of visually presented words and pseudowords. Cerebral Cortex 1996;6:62-70.

108. Pugh KR, Mencl WE, Jenner AR, et al. Functional neuroimaging studies of reading and reading disability (developmental dyslexia). Mental Retardation and Developmental Disabilities Research Reviews 2000;6:207-213.

109. Turkeltaub PE, Eden GF, Jones KM, Zeffiro TA. Meta-analysis of the functional neuroanatomy of single-word reading: Method and validation. Neuroimage 2002;16:765-780.

110. Binder JR, McKiernan KA, Parsons ME, et al. Neural correlates of lexical access during visual word recognition. Journal of Cognitive Neuroscience 2003;15:372393.

111. Puce A, Allison T, Asgari M, Gore JC, McCarthy G. Differential sensitivity of human visual cortex to faces, letterstrings, and textures: A functional magnetic resonance imaging study. Journal of Neuroscience 1996;16:5205-5215.

112. Grill-Spector K, Kourtzi Z, Kanwisher N. The lateral occipital complex and its role in object recognition. Vision Research 2001;41:1409-1422.

113. Pammer K, Hansen PC, Kringelbach ML, et al. Visual word recognition: The first half second. Neuroimage 2004;22:1819-1825.

114. Cohen L, Lehericy S, Chochon F, Lemer C, Rivaud S, Dehaene S. Languagespecific tuning of visual cortex functional properties of the visual word form area. Brain 2002;125:1054-1069. 
115. Cohen L, Dehaene S, Naccache L, et al. The visual word form area: Spatial and temporal characterization of an initial stage of reading in normal subjects and posterior split-brain patients. Brain 2000;123:291-307.

116. Dehaene S, Le Clec'H G, Poline JB, Le Bihan D, Cohen L. The visual word form area: A prelexical representation of visual words in the fusiform gyrus. Neuroreport 2002;13:321-325.

117. Polk TA, Stallcup M, Aguirre GK, et al. Neural specialization for letter recognition. Journal of Cognitive Neuroscience 2002;14:145-159.

118. Price CJ, Winterburn D, Giraud AL, Moore CJ, Noppeney U. Cortical localisation of the visual and auditory word form areas: A reconsideration of the evidence. Brain and Language 2003;86:272-286.

119. Turkeltaub PE, Gareau L, Flowers DL, Zeffiro TA, Eden GF. Development of neural mechanisms for reading. Nature Neuroscience 2003;6:767-773.

120. Cohen L, Martinaud O, Lemer C, et al. Visual word recognition in the left and right hemispheres: Anatomical and functional correlates of peripheral alexias. Cerebral Cortex 2003;13:1313-1333.

121. Hillis AE, Newhart M, Heidler J, Barker P, Herskovits E, Degaonkar M. The roles of the "visual word form area" in reading. Neuroimage 2005;24:548-559.

122. Price CJ, Devlin JT. The myth of the visual word form area. Neuroimage 2003;19:473-481.

123. Gauthier I, Tarr MJ, Moylan J, Skudlarski P, Gore JC, Anderson AW. The fusiform "face area" is part of a network that processes faces at the individual level. Journal of Cognitive Neuroscience 2000;12:495-504.

124. Van Essen DC, Zeki SM. The topographic organization of rhesus monkey prestriate cortex. Journal of Physiology 1978;193-226.

125. Binder JR, Lazar RM, Tatemichi TK, Mohr JP, Desmond DW, Ciecierski KA. Left hemiparalexia. Neurology 1992;42:562-569.

126. Beversdorf DQ, Ratcliffe NR, Rhodes $\mathrm{CH}$, Reeves AG. Pure alexia: Clinicalpathologic evidence for a lateralized visual language association cortex. Clinical Neuropathology 1997;16:328-331.

127. Palmer SL, Reddick WE, Glass JO, Gajjar A, Goloubeva O, Mulhern RK. Decline in corpus callosum volume among pediatric patients with medulloblastoma: Longitudinal MR imaging study. American Journal of Neuroradiology 2002;23:1088-1094. 
128. Schlaggar BL, Brown TT, Lugar HM, Visscher KM, Miezin FM, Petersen SE. Functional neuroanatomical differences between adults and school-age children in the processing of single words. Science 2002;296:1476-1479.

129. Tagamets MA, Novick JM, Chalmers ML, Friedman RB. A parametric approach to orthographic processing in the brain: An fMRI study. Journal of Cognitive Neuroscience 2000;12:281-297.

130. Reddick WE, White HA, Glass JO, et al. Developmental model relating white matter volume to neurocognitive deficits in pediatric brain tumor survivors. Cancer 2003;97:2512-2519.

131. Reddick WE, Russell JM, Glass JO, et al. Subtle white matter volume differences in children treated for medulloblastoma with conventional or reduced dose craniospinal irradiation. Magnetic Resonance Imaging 2000;18:787-793.

132. Kitahara S, Nakasu S, Murata K, Sho K, Ito R. Evaluation of treatment-induced cerebral white matter injury by using diffusion-tensor MR imaging: Initial experience. American Journal of Neuroradiology 2005;26:2200-2206.

133. Zou P, Phillips N, Zhang Y, et al. BOLD response in pediatric brain tumor patients during treatment. Proceedings of the International Society of Magnetic Resonance in Medicine 2005;13:441.

134. Smith SM, Johansen-Berg H, Jenkinson M, et al. Acquisition and voxelwise analysis of multi-subject diffusion data with Tract-Based Spatial Statistics. Nature Protocols 2007;2:499-503.

135. Alexander AL, Tsuruda JS, Parker DL. Elimination of eddy current artifacts in diffusion-weighted echo-planar images: The use of bipolar gradients. Magnetic Resonance in Medicine 1997;38:1016-1021.

136. Karajannis M, Allen JC, Newcomb EW. Treatment of pediatric brain tumors. Journal of Cellular Physiology 2008;217:584-589.

137. Bloom HJG, Glees J, Bell J. The treatment and long-term prognosis of children with intracranial tumors: A study of 610 cases, 1950-1981. International Journal of Radiation Oncology Biology Physics 1990;18:723-745.

138. Nieuwenhuys R, Voogd J, van Huizen C. The human central nervous system, 4th ed. Berlin, Heidelberg, New York: Springer-Verlag, 2008.

139. Pandya DN, Barbas H. Architecture and connections of the premotor areas in the rhesus-monkey. Behavioral and Brain Sciences 1985;8:595-596.

140. Vanhoesen GW, Rosene DL, Mesulam MM. Subicular input from temporal cortex in the rhesus-monkey. Science 1979;205:608-610. 
141. Braak H, Braak E. Evolution of neuronal changes in the course of Alzheimer's disease. Journal of Neural Transmission-Supplement 1998;127-140.

142. Tanji J, Shima K, Matsuzaka Y. Reward-based planning of motor selection in the rostral cingulate motor area. Sensorimotor Control of Movement and Posture 2002;508:417-423.

143. He SQ, Dum RP, Strick PL. Topographic organization of corticospinal projections from the frontal-lobe: Motor areas on the lateral surface of the hemisphere. Journal of Neuroscience 1993;13:952-980.

144. Hatanaka N, Tokuno H, Hamada I, et al. Thalamocortical and intracortical connections of monkey cingulate motor areas. Journal of Comparative Neurology 2003;462:121-138.

145. Rees G. Visual Attention: The thalamus at the centre? Current Biology 2009;19:R213-R214.

146. Zhang Y, Zou P, Mulhern R, Butler R.W., Laningham FH, Ogg RJ. Brain structural abnormalities in survivors of pediatric posterior fossa brain tumors: A voxel-based morphometry study using free-form deformation. Neuroimage 2008;42[1]: 218-229.

147. Kastner S, O'Connor DH, Fukui MM, Fehd HM, Herwig U, Pinsk MA. Functional imaging of the human lateral geniculate nucleus and pulvinar. Journal of Neurophysiology 2004;91:438-448.

148. McAlonan K, Cavanaugh J, Wurtz RH. Guarding the gateway to cortex with attention in visual thalamus. Nature 2008;456:391-U56.

149. Adams MM, Hof PR, Gattass R, Webster MJ, Ungerleider LG. Visual cortical projections and chemoarchitecture of macaque monkey pulvinar. Journal of Comparative Neurology 2000;419:377-393.

150. Maunsell JHR, Vanessen DC. Functional-properties of neurons in middle temporal visual area of the macaque monkey. Journal of Neurophysiology 1983;49:1148-1167.

151. Asanuma C, Stanfield BB. Medial leminscal axons can innervate the lateral geniculate-nucleus in neonatally enucleated and congenitally blind mice. Anatomical Record 1985;211:A12.

152. Huerta MF, Krubitzer LA, Kaas JH. Frontal eye field as defined by intracortical microstimulation in squirrel-monkeys, owl monkeys, and macaque monkeys: Subcortical connections. Journal of Comparative Neurology 1986;253:415-439.

153. Kastner S, Ungerleider LG. Mechanisms of visual attention in the human cortex. Annual Review of Neuroscience 2000;23:315-341. 
154. Karnath HO, Himmelbach M, Rorden C. The subcortical anatomy of human spatial neglect: Putamen, caudate nucleus and pulvinar. Brain 2002;125:350-360.

155. Khong PL, Leung LHT, Fung ASM, et al. White matter anisotropy in posttreatment childhood cancer survivors: Preliminary evidence of association with neurocognitive function. Journal of Clinical Oncology 2006;24:884-890.

156. Nagy Z, Westerberg H, Klingberg T. Maturation of white matter is associated with the development of cognitive functions during childhood. Journal of Cognitive Neuroscience 2004;16:1227-1233.

157. Greig NH, Soncrant TT, Shetty HU, Momma S, Smith QR, Rapoport SI. Brain uptake and anticancer activities of vincristine and vinblastine are restricted by their low cerebrovascular permeability and binding to plasma constituents in rat. Cancer Chemotherapy and Pharmacology 1990;26:263-268.

158. Baillieux H, De Smet HJ, Paquier PF, De Deyn PP, Marien P. Cerebellar neurocognition: Insights into the bottom of the brain. Clinical Neurology and Neurosurgery 2008;110:763-773.

159. Schmahmann JD. Disorders of the cerebellum: Ataxia, dysmetria of thought, and the cerebellar cognitive affective syndrome. Journal of Neuropsychiatry and Clinical Neurosciences 2004;16:367-378.

160. Schmahmann JD, Pandya DN. The cerebrocerebellar system. Cerebellum and Cognition 1997;41:31-60.

161. Baillieux H, Weyns F, Paquier P, De Deyn PP, Marien P. Posterior fossa syndrome after a vermian stroke: A new case and review of the literature. Pediatric Neurosurgery 2007;43:386-395.

162. Chugani DC, Muzik O, Rothermel R, et al. Altered serotonin synthesis in the dentatothalamocortical pathway in autistic boys. Annals of Neurology 1997;42:666-669.

163. Muller RA, Chugani DC, Behen ME, et al. Impairment of dentato-thalamocortical pathway in autistic men: Language activation data from positron emission tomography. Neuroscience Letters 1998;245:1-4.

164. Martin P, Albers M. Cerebellum and schizophrenia: A selective review. Schizophrenia Bulletin 1995;21:241-250.

165. Aguiar PH, Plese JPP, Ciquini O, Marine R. Transient mutism following a posterior-fossa approach to cerebellar tumors in children: A critical-review of the literature. Childs Nervous System 1995;11:306-310.

166. Gelabert-Gonzalez M, Fernandez-Villa J. Mutism after posterior fossa surgery. Review of the literature. Clinical Neurology and Neurosurgery 2001;103:111-114. 
167. Wells EM, Walsh KS, Khademian ZP, Keating RF, Packer RJ. The cerebellar mutism syndrome and its relation to cerebellar cognitive function and the cerebellar cognitive affective disorder. Developmental Disabilities Research Reviews 2008;14:221-228.

168. Robertson PL, Muraszko KM, Holmes EJ, et al. Incidence and severity of postoperative cerebellar mutism syndrome in children with medulloblastoma: A prospective study by the Children's Oncology, Group. Journal of Neurosurgery 2006; 105:444-451.

169. Doxey D, Bruce D, Sklar F, Swift D, Shapiro K. Posterior fossa syndrome: Identifiable risk factors and irreversible complications. Pediatric Neurosurgery 1999;31:131-136.

170. Huber JF, Bradley K, Spiegler BJ, Dennis M. Long-term effects of transient cerebellar mutism after cerebellar astrocytoma or medulloblastoma tumor resection in childhood. Childs Nervous System 2006;22:132-138.

171. Steinbok P, Cochrane DD, Perrin R, Price A. Mutism after posterior fossa tumour resection in children: Incomplete recovery on long-term follow-up. Pediatric Neurosurgery 2003;39:179-183.

172. Steinlin M, Imfeld S, Zulauf $\mathrm{P}$, et al. Neuropsychological long-term sequelae after posterior fossa tumour resection during childhood. Brain 2003;126:1998-2008.

173. Catsman-Berrevoets CE, Van Dongen HR, Mulder PGH, Geuze DPY, Paquier $\mathrm{PF}$, Lequin $\mathrm{MH}$. Tumour type and size are high risk factors for the syndrome of "cerebellar" mutism and subsequent dysarthria. Journal of Neurology Neurosurgery and Psychiatry 1999;67:755-757.

174. Kusano Y, Tanaka Y, Takasuna H, et al. Transient cerebellar mutism caused by bilateral damage to the dentate nuclei after the second posterior fossa surgery: Case report. Journal of Neurosurgery 2006;104:329-331.

175. Ozgur BM, Berberian J, Aryan HE, Meltzer HS, Levy ML. The pathophysiologic mechanism of cerebellar mutism. Surgical Neurology 2006;66:18-25.

176. Levisohn L, Cronin-Golomb A, Schmahmann JD. Neuropsychological consequences of cerebellar tumour resection in children: Cerebellar cognitive affective syndrome in a paediatric population. Brain 2000;123:1041-1050.

177. Riva D, Giorgi C. The cerebellum contributes to higher functions during development: Evidence from a series of children surgically treated for posterior fossa tumours. Brain 2000;123:1055-1061.

178. Reese TG, Heid O, Weisskoff RM, Wedeen VJ. Reduction of eddy-currentinduced distortion in diffusion MRI using a twice-refocused spin echo. Magnetic Resonance in Medicine 2003;49:177-182. 
179. Ersahin Y, Mutluer S, Cagli S, Duman Y. Cerebellar mutism: Report of seven cases and review of the literature. Neurosurgery 1996;38:60-66.

180. Frassanito P, Massimi L, Caldarelli M, Di Rocco C. Cerebellar mutism after spontaneous intratumoral bleeding involving the upper cerebellar vermis: A contribution to the physiopathogenic interpretation. Childs Nervous System 2009;25:7-11.

181. Kotil K, Eras M, Akcetin M, Bilge T. Cerebellar mutism following posterior fossa tumor resection in children. Turkish Neurosurgery 2008;18:89-94.

182. Crutchfield JS, Sawaya R, Meyers CA, Moore BD. Postoperative mutism in neurosurgery: Report of 2 cases. Journal of Neurosurgery 1994;81:115-121.

183. Koh S, Turkel SB, Baram TZ. Cerebellar mutism in children: Report of six cases and potential mechanisms. Pediatric Neurology 1997;16:218-219.

184. Miller N, Kocak M, Glass J, et al. MRI Evaluation of the proximal efferent cerebellar pathway in pediatric patients with posterior fossa syndrome. Manuscript submitted for publication, 2009.

185. Marien P, Saerens J, Nanhoe R, et al. Cerebellar induced aphasia: Case report of cerebellar induced prefrontal aphasic language phenomena supported by SPECT findings. Journal of the Neurological Sciences 1996;144:34-43.

186. Clerico A, Sordi A, Ragni G, Festa A, Cappelli C, Maini CL. Transient mutism following posterior fossa surgery studied by Single Photon Emission Computed Tomography (SPECT). Medical and Pediatric Oncology 2002;38:445-448.

187. Ersahin Y, Yararbas U, Duman Y, Mutluer S. Single photon emission tomography following posterior fossa surgery in patients with and without mutism. Childs Nervous System 2002;18:318-325.

188. Sagiuchi T, Ishii K, Aoki Y, et al. Bilateral crossed cerebello-cerebral diaschisis and mutism after surgery for cerebellar medulloblastoma. Annals of Nuclear Medicine 2001;15:157-160.

189. Glickstein M. Thinking about the cerebellum. Brain 2006;129:288-290.

190. Morgane PJ, Galler JR, Mokler DJ. A review of systems and networks of the limbic forebrain/limbic midbrain. Progress in Neurobiology 2005;75:143-160. 


\section{Appendix A. Modified SPM99 Poldrack Diffusion Calculation}

$\%$ function spm_diffusion_calc(images,b)

$\%$ spm_diffusion_calc: part of the SPM diffusion tensor toolbox

$\%$ Russ Poldrack, 11/19/00

$\%$ This program is modified by Dr. R Ogg, PZou and NPhillips (Feb 2003).

$\%$ gradient pulse sequence (an interleave single-shot multisection spin-echo EPI),

$\%$ which has one no-gradient, six diffusion gradients.

$\%$

$\%$ This program was also modified by P Zou, and N Phillips (Feb 2003) by vectorizing

$\%$ the base code to take advantage of MATLAB processing.

$\%$

$\%$ The ep2d_diff sequence can neglect spoilers or RO pre-de-phasing gradients. It also

$\%$ can not be expect substantial contributions from the de- and re-phasing parts, because

$\%$ the time between them is short.

$\%$

$\%$ - This routine calculates the diffusion tensor and fractional

$\%$ anisotropy based upon a set of 6 diffusion-weighted images along

$\%$ with a non-diffusion-weighted (low-b) image. The calculation is based on code

$\%$ provided by Mette Weigell at the MGH-NMR Center.

$\%$

$\%$

$\%$ Arguments:

$\%$ images: this should specify a set of 7 images, with the following

$\%$ diffusion weightings (images must in this specific order):

$\%$

$\begin{array}{llll}\% & X & Y & Z\end{array}$

$\% 1$ - $0 \quad 0 \quad 0$ (i.e., no diffusion weighting)

$\% 2-1 \quad 0 \quad 1$

$\% \begin{array}{llll}\% & -1 & 0 & 1\end{array}$

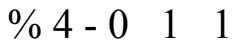

$\% 5-0-1 \quad 1$

$\% 6-1 \quad 1 \quad 0$

$\% 7-\begin{array}{lll}\% & 1 & 0\end{array}$

$\%$

$\%$ b: b value (i.e., amount of diffusion weighting)

$\%$

$\%$ Effects:

$\%$ creates the following files:

$\%$ adc.img - Apparent diffusion coefficient calculated from trace

$\%$ fa.img - fractional anisotropy

$\%$ col1_[1-3].img - images representing the R,G,and B components respectively

$\%$ of a diffusion colormap for the first eigenvector of the tensor

$\%$ col3_[1-3].img - images representing the R,G,and B components respectively 
$\%$ of a diffusion colormap for the third eigenvector of the tensor

$\%$ eig1_ $\{X, Y, Z\}$.img - images representing the $\mathrm{X}, \mathrm{Y}$, and $\mathrm{Z}$ components of the

$\%$ first eigenvector respectively.

$\%$ eig.bfloat - binary image containing tensor and eigenvector information

$\%$ (for use with in-house MGH tools)

$\%$

$\%$ CVS repository and development information can be found at:

$\%$ http://spm-toolbox.sourceforge.net

$\%$

$\%$ The SPM diffusion toolbox is distributed freely under the GNU Public License.

$\%$

vols $=$ spm_vol(images);

[data XYZ] $=$ spm_read_vols(vols);

$\mathrm{a}=$ zeros(size(data));

$\mathrm{a}(:,:,:, 1: 6)=\operatorname{data}(:,:,:, 2: 7)$;

$\mathrm{a}(:,:,:, 7)=$ data(::,::,1); \% put low-b data into 7

$\%$ set up b matrix

if isempty('b'),b=1000;

end;

$\% \mathrm{a}=$ squeeze(reshape(buff,[128 $1287 \mathrm{nslc}])$ );

[xsize,ysize, $n s l c$, foo] $=$ size $(a)$;

$\%$ note - need to switch $\mathrm{k}$ and 1 indices

$\% \%$ FOR DEBUGGING ONLY

tmpx=zeros(xsize,ysize,nslc,6);

lowb_thresh $=50$;

spm_progress_bar('Init',nslc*2);

spm('Pointer','Watch');

prog_counter $=0$;

for $\mathrm{k}=1$ :nslc

fprintf('calculating diffusion coefficients for slice $\# \% \mathrm{~d} \backslash \mathrm{n}$ ',k);

$\%$ imagesc $(\mathrm{a}(:,:, \mathrm{k}, 1))$;

for $\mathrm{i}=1$ :xsize

for $\mathrm{j}=1$ :ysize;

warning off;

for $1=1: 7$

$\operatorname{if}(\operatorname{isinf}(a(i, j, k, l)) \mid \operatorname{isnan}(a(i, j, k, l))) a(i, j, k, l)=0$; end

end;

if $\mathrm{a}(\mathrm{i}, \mathrm{j}, \mathrm{k}, 7)>$ lowb_thresh

for $1=1: 6$ 


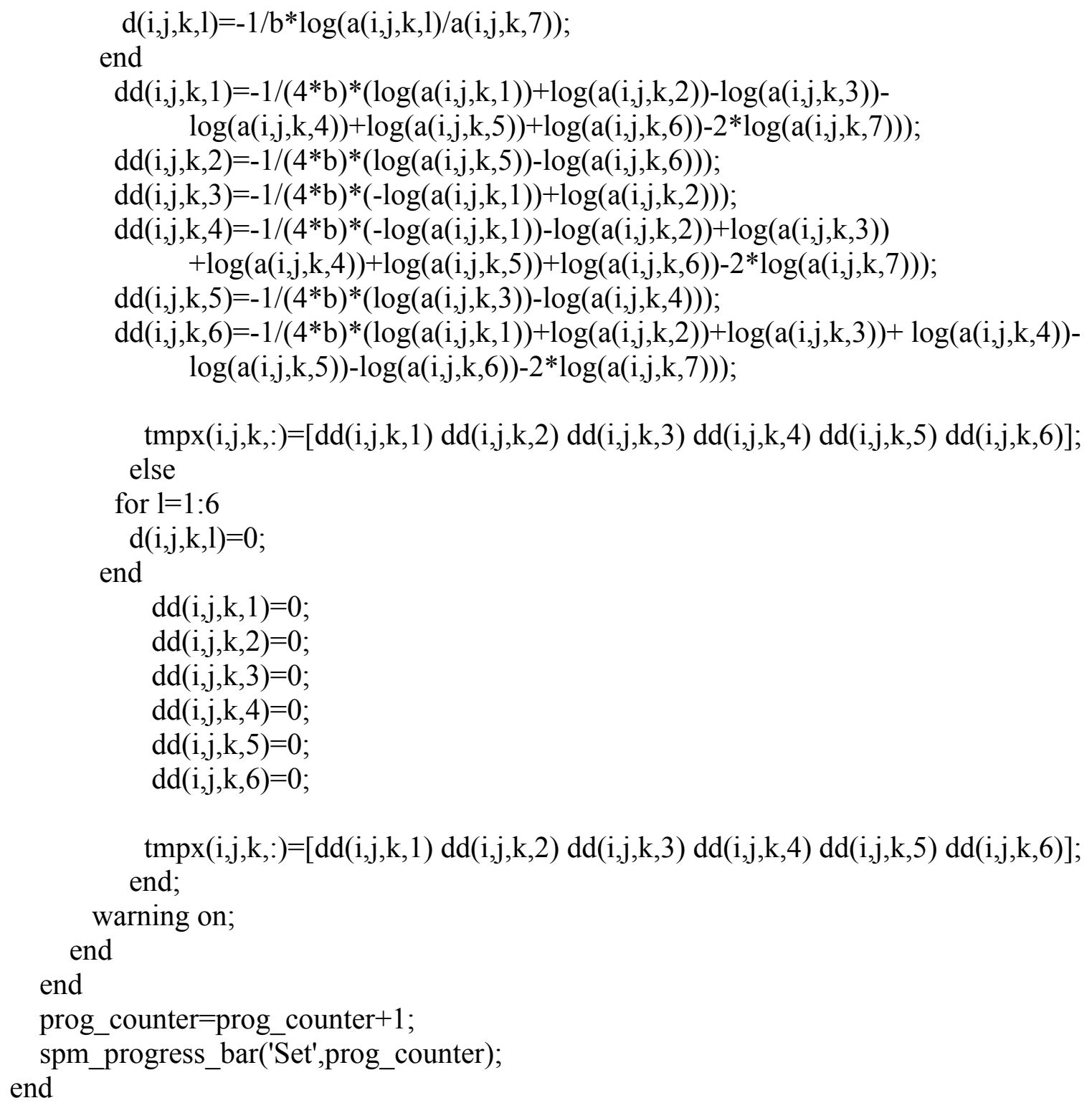




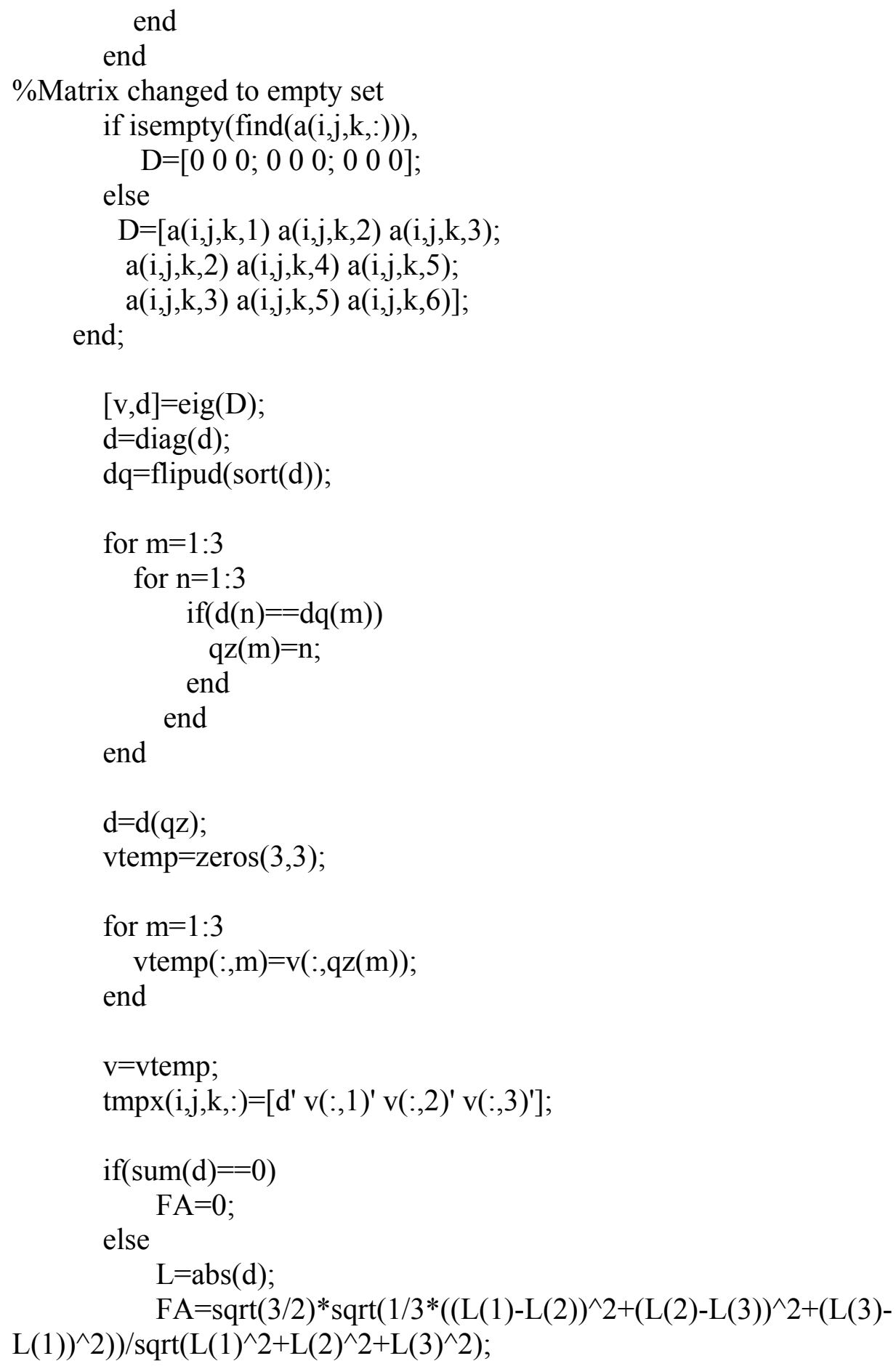




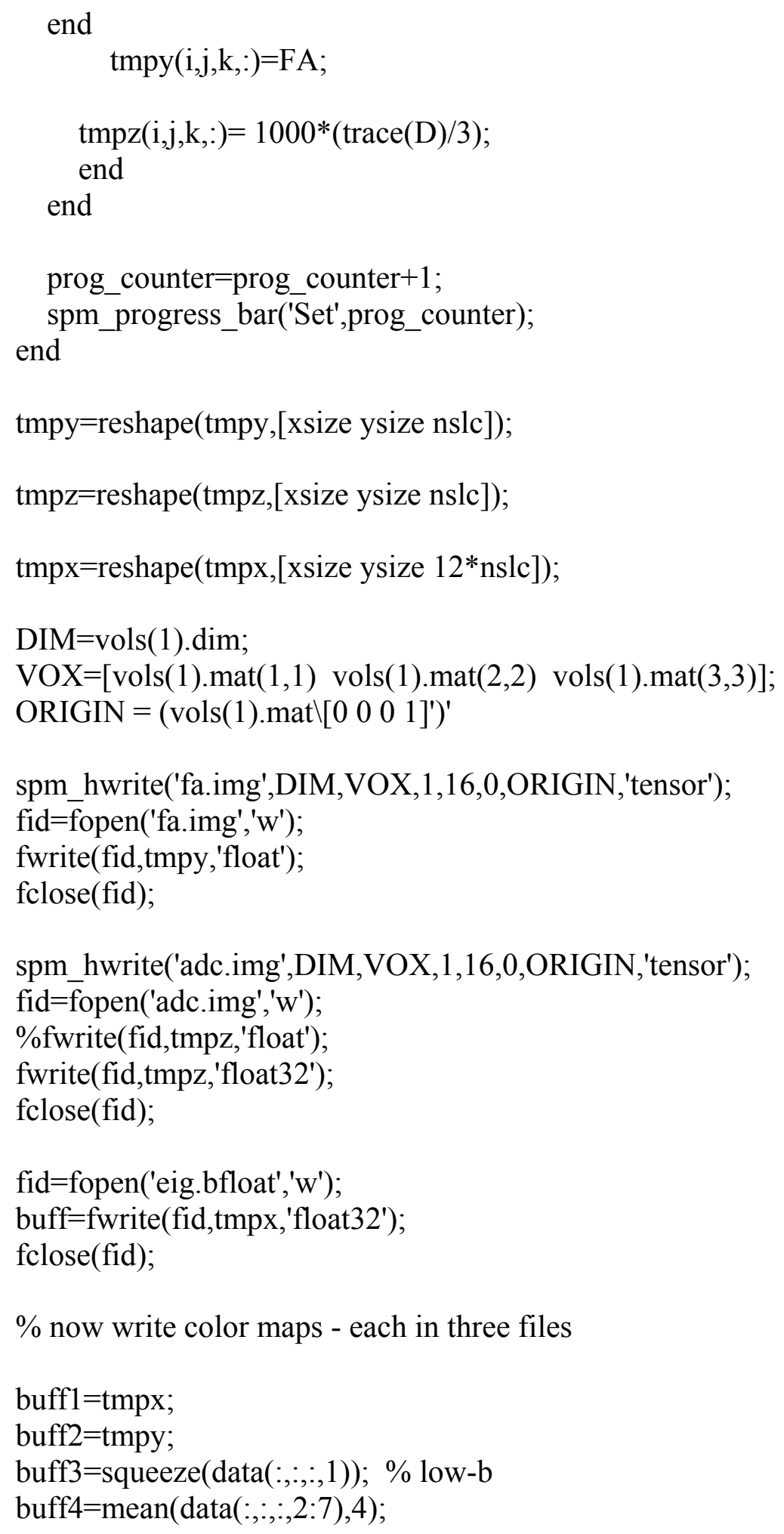

imdeck=squeeze $($ reshape $($ buff1, $[$ xsize ysize $12 *$ nslc $]))$; 
imdeck=permute(imdeck, $[2,1,3])$;

[npix,nim,imd] $=$ getimd(imdeck);

imdeck2=squeeze(reshape(buff2,[xsize ysize nslc]));

imdeck2=permute(imdeck2,[2,1,3]);

[npix,nim,imd] =getimd(imdeck2);

imdeck3=squeeze(reshape(buff3,[xsize ysize nslc]));

imdeck3=permute(imdeck3,[2,1,3]);

[npix,nim,imd] =getimd(imdeck3);

imdeck4=squeeze(reshape(buff4,[xsize ysize nslc]));

imdeck4=permute(imdeck4,[2,1,3]);

[npix,nim,imd] =getimd(imdeck3);

npar $=12$

$\mathrm{n}=$ nslc*npar;

npar2=1;

n2=nslc*npar2;

$\mathrm{L} 1=\operatorname{imdeck}(:,:, 1: \mathrm{nslc})$;

L2=imdeck $(:,:, n s l c+1: 2 *$ nslc $)$;

L3 $=\operatorname{imdeck}(:,:, 2 *$ nslc $+1: 3 *$ nslc $)$;

$\mathrm{E} 1 \mathrm{x}=\operatorname{imdeck}(:,:, 3 *$ nslc $+1: 4 *$ nslc $)$;

$\mathrm{E} 1 \mathrm{y}=\operatorname{imdeck}(:,:, 4 * \mathrm{nslc}+1: 5 *$ nslc $)$;

E1z=imdeck $(:,:, 5 *$ nslc $+1: 6 *$ nslc $)$;

$\mathrm{E} 3 \mathrm{x}=\operatorname{imdeck}(:,:, 6 *$ nslc $+1: 7 *$ nslc $)$;

$\mathrm{E} 3 \mathrm{y}=\operatorname{imdeck}(:,:, 7 *$ nslc $+1: 8 *$ nslc $)$;

$\mathrm{E} 3 \mathrm{z}=\operatorname{imdeck}(:,:, 8 *$ nslc $+1: 9 *$ nslc $)$;

$\mathrm{FA}=$ imdeck2(:,:,:);

lowb=imdeck3(:,:::);

DWI=imdeck4(:,:,:);

Df2=abs(L2-L3);

FAmax $=\max ($ reshape $(F A, n p i x, n s l c))$;

FAmax=reshape(repmat(FAmax,npix,1),[imd(1) imd(2) nslc]);

FAscaled $=$ FA./FAmax;

trace $=\operatorname{abs}(\mathrm{L} 1+\mathrm{L} 2+\mathrm{L} 3)$;

$\%$ mask $=\left((\right.$ lowb $>10) \&($ FAscaled $<1) \&\left(\right.$ trace $\left.\left.<3.5^{*} 10^{\wedge}-3\right)\right)$;

mask $=\left((\right.$ lowb $>10) \&(\mathrm{FA}<1) \&\left(\right.$ trace $\left.\left.<3.5^{*} 10^{\wedge}-3\right)\right)$; 


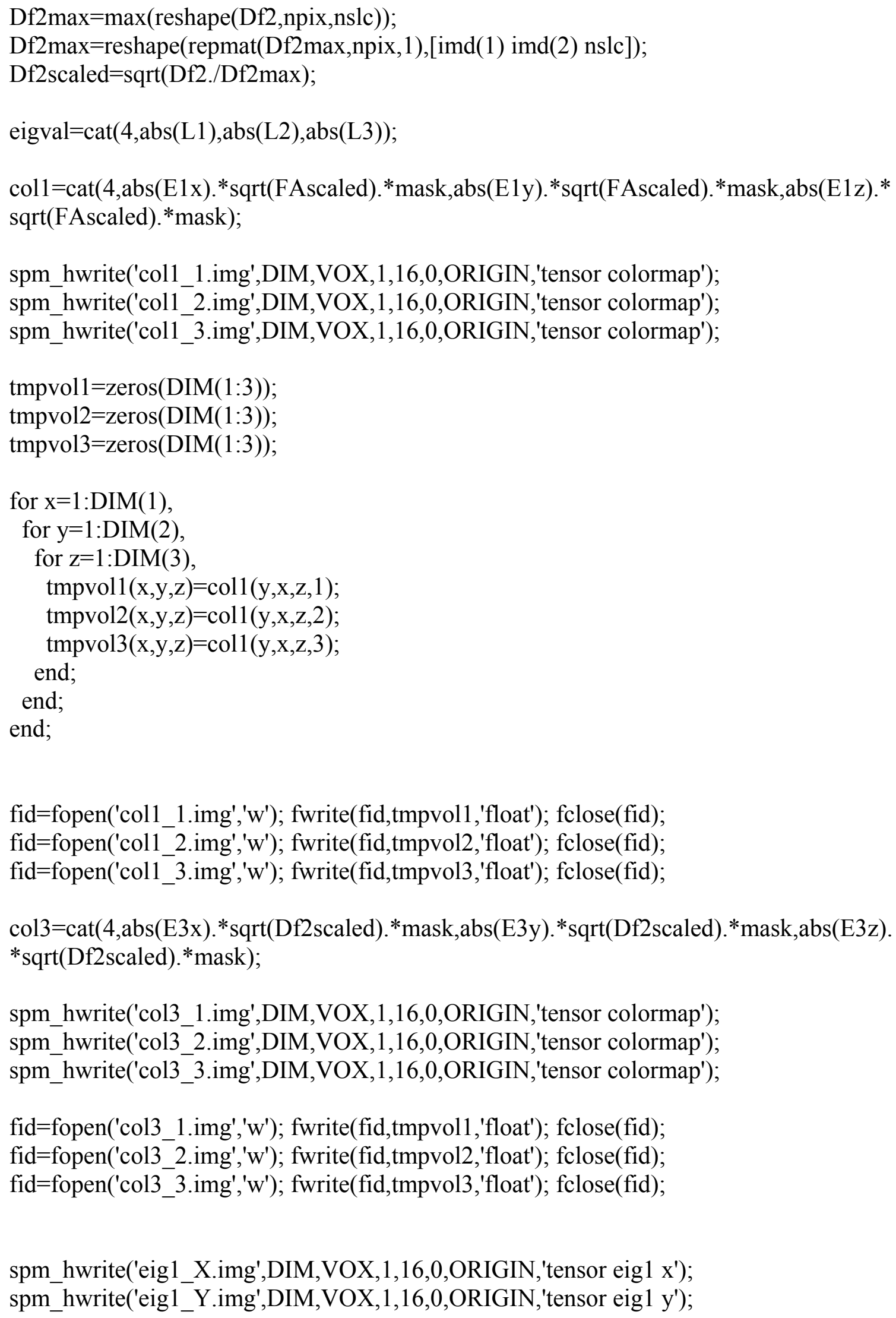

spm_hwrite('eig1_X.img',DIM,VOX,1,16,0,ORIGIN,'tensor eig1 x'); spm_hwrite('eig1_Y.img',DIM,VOX,1,16,0,ORIGIN,'tensor eig1 y'); 
spm_hwrite('eig1_Z.img',DIM,VOX,1,16,0,ORIGIN,'tensor eig1 z');

tmpvolx=zeros(DIM(1:3));

tmpvoly=zeros(DIM(1:3));

tmpvolz=zeros(DIM(1:3));

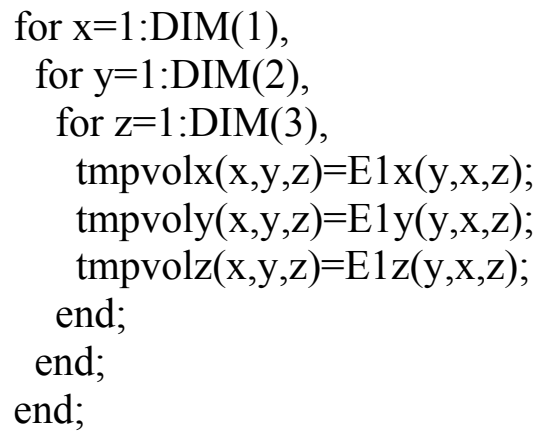

fid=fopen('eig1_X.img','w'); fwrite(fid,tmpvolx,'float'); fclose(fid); fid=fopen('eig1_Y.img','w'); fwrite(fid,tmpvoly,'float'); fclose(fid); fid=fopen('eig1_Z.img','w'); fwrite(fid,tmpvolz,'float'); fclose(fid);

spm_progress_bar('Clear');

spm('Pointer','Arrow'); 


\section{Appendix B. SPM2 DTI Preprocessing Toolbox}

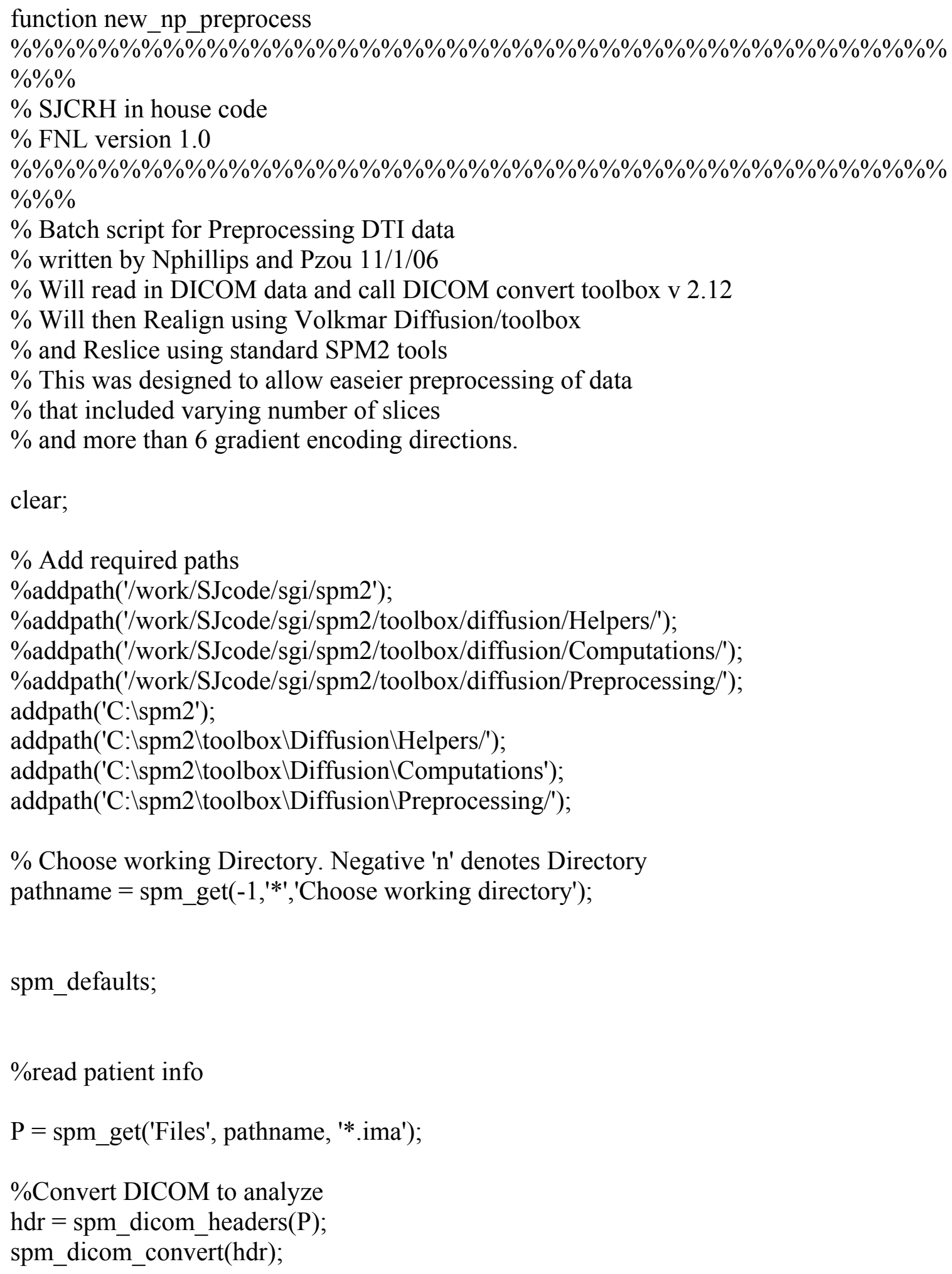


$\%$ Realign

$\%$ FORMAT bchdone $=$ dti_realign $(\mathrm{bch})$

$\%====$

$\%$ Input argument:

$\%$ bch - struct array of batch descriptions with fields

$\% \quad$.files - cell array of image filenames

$\% \quad$ or struct with elements

$\%$.dirs - cell array of directory names

$\% \quad$.filter - filter string

$\% \quad$ The .files field will be filled by calling

$\% \quad$ spm_get('files',bch.files.dirs $\{\mathrm{k}\}, \mathrm{bch}$. files.filter)

$\% \quad$ and concatenating the resulting file name arrays.

$\% \quad$ Images are classified as b0 or b1 by inspection of

$\%$ the diffusion information in userdata.

$\%$.b0corr - Motion correction on b0 images

$\% \quad 0$ - None

$\% \quad 1-$ Stepwise

$\% \quad 2$ - Linear, last stepwise

$\% \quad 3$ - Linear, last linear

$\%$.blcorr - Motion correction on b1 images

$\% \quad 0$ - None

$\% \quad 1-$ Realign xy

$\% \quad 2$ - Realign each diffusion weighting separately

$\% \quad$ (may be used without b0 motion correction)

$\% \quad 3$ - Realign $x y$, then coregister to mean

$\%$ Output argument:

$\%$ bchdone - filled batch structure.

$\%$ bch(subj).files.dirs $\{1\}=$ pathname ;

$\%$ bch(subj).files.filter='s*.img';

Apathname $=$ [pathname, '\analyzedata']; \% \% 11-08-06 pz \%\%\% 11-09-06 nsp

$\mathrm{PP}=$ spm_get('Files', Apathname, 's*.img'); \%\% 11-08-06 pz

bch.files $=$ cellstr(PP); $\quad \% \% \quad 11-08-06 \mathrm{pz}$

bch.b0corr $=2$;

bch.b1 corr $=1$;

bchdone $=$ dti_realign $(\mathrm{bch})$;

$\%$ Reslicing

disp(sprintf('subject \%d, reslicing...', pathname))

spm_reslice $(\mathrm{PP})$;

$\% \% \quad 11-08-06 \mathrm{pz}$ 
$\%$ Add Mask

$\%$ spm_create_mask1(PP(1),45);

clear; 


\section{Appendix C. Modified SPM2 (Volkmar) DICOM Convert Files}

function spm_dicom_convert(hdr,opts,root_dir)

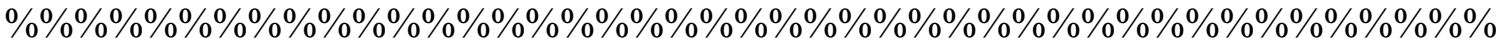

$\% \% \% \% \% \% \% \% \% \%$

$\%$ SJCRH in house code

$\%$ FNL version 2.2

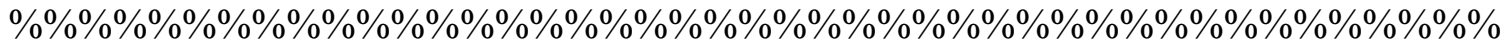

$\% \% \% \% \% \% \% \% \% \%$

$\%$ Convert DICOM images into something that SPM can use

$\%$ FORMAT spm_dicom_convert(hdr,opts)

$\%$ hdr - a cell array of DICOM headers from spm_dicom_headers

$\%$ opts - options

$\% \quad$ 'all' - all DICOM files (default)

$\% \quad$ 'mosaic' - the mosaic images

$\% \quad$ 'standard' - standard DICOM files

$\%$ 'raw' - convert raw FIDs (not implemented)

$\%$

$\%$ Converted files are written to the current directory

$\%$

\% @ line 477-485 and 488-493 pzou 11/8/08 allow output files to be written

$\%$ in working directory. Allows SJCRH FNL batch preprocessing program

$\%$ compatibility.

\%@ line 789: \% nsp 30oct2006 correct z and y flip problem in vector information

\%@ line 462 11/08/06 PZ reasigned dname to a fixed directory (analyzedata), and file

name include series \#

\%@(\#)Hacked by VG to read 128p*128 AcquisitionMatrixText

$\% @$ @\#)Hacked by VG to sort volumes by ICE_dims and read diffusion information

$\%$ diffusion information: flip y direction of gradient vector

\%@(\#)spm_dicom_convert.m 2.17 John Ashburner 03/10/22

$\%$

$\%$ This version has been hacked to allow for conversion of

$\%$ diffusion weighted EPI data acquired with the GE scanners

$\%$ at MRC, Karolinska, Stockholm.

$\%$ The problem with these data is that there is NOTHING in

$\%$ the DICOM headers to distinguish the different volumes

$\%$ acquired with different diffusion gradients. The result

$\%$ is that all slices from the entire dwi/DTI protocol get

$\%$ squeezed into a single volume. This volume will then have

$\%$ multiple slices with the same z-position, and will (in the

$\%$ original version of spm_dicom_convert) cause a warning.

$\%$ I have "solved" this by adding an additional branch to

$\%$ be executed if we have multiple slices at the same location

$\%$ AND data have been acquired on a GE scanner. 
$\%$ Its not pretty, but most of us aren't.

$\%$

$\overline{\%}$ Jesper Andersson

if $\operatorname{nargin}<2$, opts = 'all'; end;

if nargin $<3$, root_dir='study'; end;

[images,guff] = select_tomographic_images(hdr);

[mosaic,standard] = select_mosaic_images(images);

if (strcmp(opts,'all') | strcmp(opts,'mosaic')) \& isempty(mosaic), convert_mosaic(mosaic,root_dir);

end;

if (strcmp(opts,'all') | strcmp(opts,'standard')) \& isempty(standard), convert_standard(standard,root_dir);

end;

return;

$\%$

$-$

$\%$

function convert_raw(hdr)

$\operatorname{disp}\left({ }^{* * *}\right.$ Ignoring Raw Data DICOM Files ***');

return;

$\%$

$-$

$\%$

function convert_mosaic(hdr,root_dir)

spm_progress_barr('Init',length(hdr), ['Writing Mosaic'], 'Files written');

for $\mathrm{i}=1$ :length(hdr),

$\%$ Output filename

\%-----------------------------------------------------------------------

$\%$ fname $=$ sprintf('f\%s-\%.4d-\%.5d-\%.6d.img', strip_unwanted(hdr $\{\mathrm{i}\}$. PatientID), ,..

$\% \operatorname{hdr}\{i\}$.SeriesNumber, $h d r\{i\}$.AcquisitionNumber, hdr $\{i\}$.InstanceNumber);

if isfield(hdr $\{$ i $\}$,'ProtocolName')

hdr $\{$ i $\}$.ProtocolName='unknown';

end;

switch root_dir

case 'study',

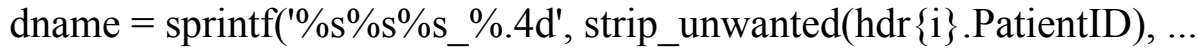


case 'patient',

filesep, strip_unwanted(hdr $\{i\}$.ProtocolName), ...

hdr $\{i\}$.SeriesNumber);

dname $=\operatorname{sprintf}\left(\% \mathrm{os} \% \mathrm{~s} \% \mathrm{~s} \% \mathrm{~s} \% \mathrm{~s} \_\% .4 \mathrm{~d}\right.$ ', strip_unwanted(hdr $\{\mathrm{i}\}$. PatientsName $), \ldots$

filesep, strip_unwanted(hdr $\{$ i $\}$.PatientID), ...

filesep, strip_unwanted(hdr $\{\mathrm{i}\}$.ProtocolName), ...

otherwise hdr $\{i\}$.SeriesNumber);

error('unknown file root specification');

end;

fname $=\operatorname{sprintf('f\% s-\% .5d-\% .5d.img',~strip\_ unwanted(hdr~}\{\mathrm{i}\}$. PatientID), ...

hdr $\{\mathrm{i}\}$.AcquisitionNumber, $\mathrm{hdr}\{\mathrm{i}\}$.InstanceNumber);

if exist(fullfile(pwd,dname)) $\sim=7$

mkdir_rec(dname);

end;

$\%$ Image dimensions and data

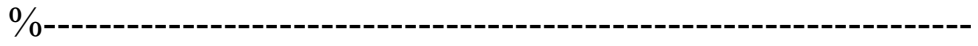

$\mathrm{nc}=\mathrm{hdr}\{\mathrm{i}\}$.Columns;

$\mathrm{nr}=\mathrm{hdr}\{\mathrm{i}\}$.Rows;

$\mathrm{np}=$ read_AcquisitionMatrixText(hdr $\{\mathrm{i}\})$;

$\%$ fix for rectangular mosaics

if $\operatorname{rem}(\mathrm{nc}, \mathrm{np}(1)) \mid \operatorname{rem}(\mathrm{nr}, \mathrm{np}(2))$

$n p=n p([21]) ; \%$ try other way round

if $\operatorname{rem}(\mathrm{nc}, \mathrm{np}(1)) \mid \operatorname{rem}(\mathrm{nr}, \mathrm{np}(2))$,

warning(sprintf('\%s: \%dx\%d wont fit into \%dx\%d.',hdr $\{\mathrm{i}\}$.Filename,...

$\mathrm{np}(1), \mathrm{np}(2), \mathrm{nc}, \mathrm{nr}))$;

return;

end;

end;

$\operatorname{dim}=$ [np read_NumberOfImagesInMosaic $($ hdr $\{\mathrm{i}\})]$;

mosaic $=$ read_image_data $(\operatorname{hdr}\{i\})$;

volume $=\operatorname{zeros}(\operatorname{dim})$;

for $\mathrm{j}=1: \operatorname{dim}(3)$,

img $=$ mosaic $((1: n p(1))+n p(1) * \operatorname{rem}(j-1, n c / n p(1)),(n p(2):-1: 1)+n p(2) *$ floor $((j-$

1)/(nc/np(1))));

if $\sim \operatorname{any}(\operatorname{img}(:))$,

volume $=$ volume $(:,:, 1:(\mathrm{j}-1))$;

break;

end;

volume $(:,:, j)=\mathrm{img}$;

end;

$\operatorname{dim}=[\operatorname{size}($ volume $)$ spm_type('int 16') $]$;

$\%$ Orientation information 


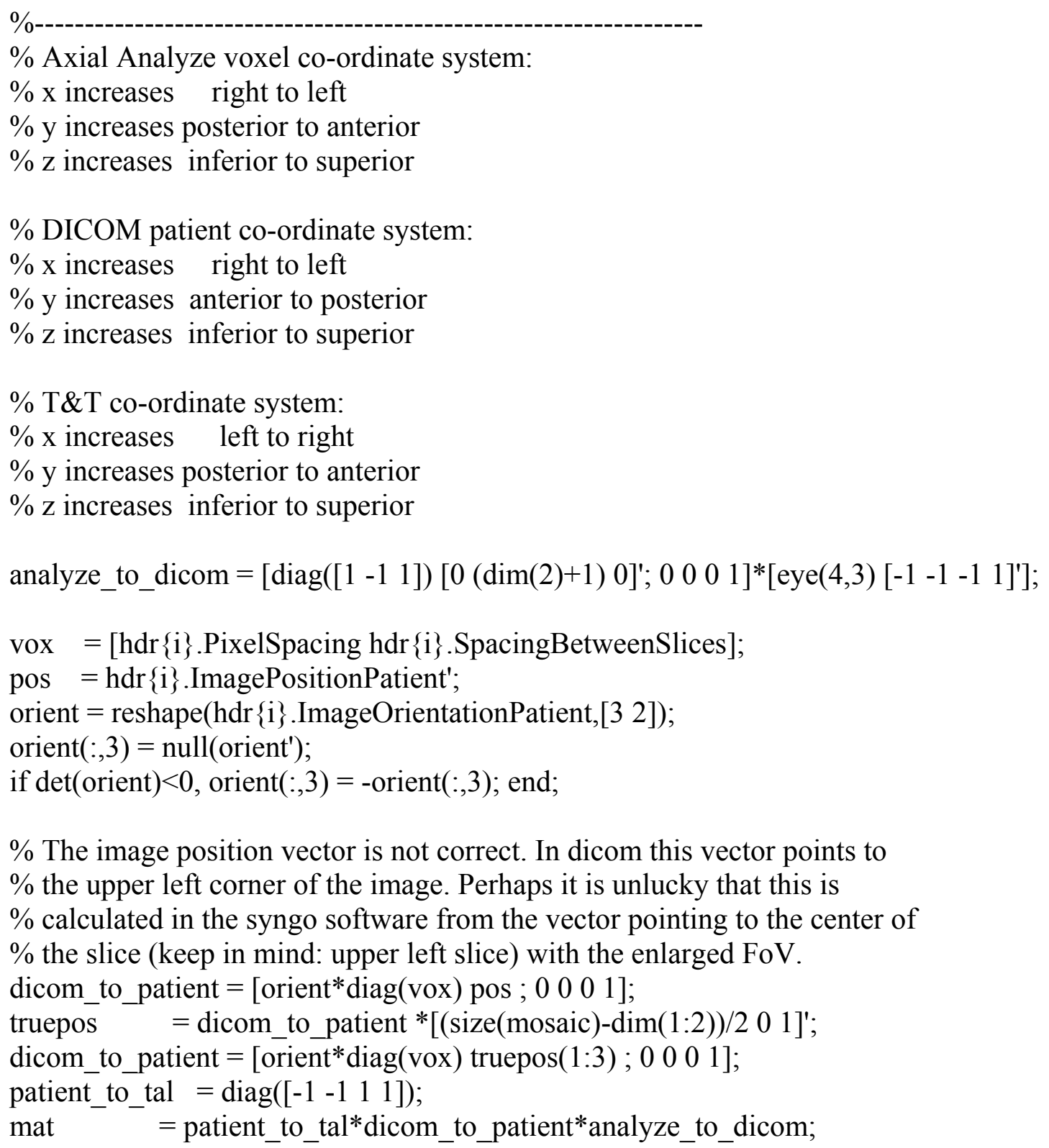

$\%$ The image position vector is not correct. In dicom this vector points to $\%$ the upper left corner of the image. Perhaps it is unlucky that this is $\%$ calculated in the syngo software from the vector pointing to the center of $\%$ the slice (keep in mind: upper left slice) with the enlarged FoV. dicom_to_patient $=\left[\right.$ orient $* \operatorname{diag}(\operatorname{vox})$ pos $\left.; \begin{array}{llll}0 & 0 & 0 & 1\end{array}\right]$;

$\%$ Maybe flip the image depending on SliceNormalVector from 0029,1010

\%liceNormalVector $=$ read SliceNormalVector(hdr $\{i\})$.

SliceNormalVector $=$ read_SliceNormalVector(hdr $\{\mathrm{i}\})$;

if $\operatorname{det}([\operatorname{reshape}(\mathrm{hdr}\{\mathrm{i}\}$.ImageOrientationPatient,[3 2]) SliceNormalVector(:)])<0; volume $=$ volume $(:,:$, end:-1:1); mat $=$ mat $^{*}\left[\operatorname{eye}(3)\left[\begin{array}{llll}0 & 0 & -(\operatorname{dim}(3)-1)\end{array}\right]\right.$; $\left.\begin{array}{lllll}0 & 0 & 0 & 1\end{array}\right]$; end;

$\%$ Possibly useful information 


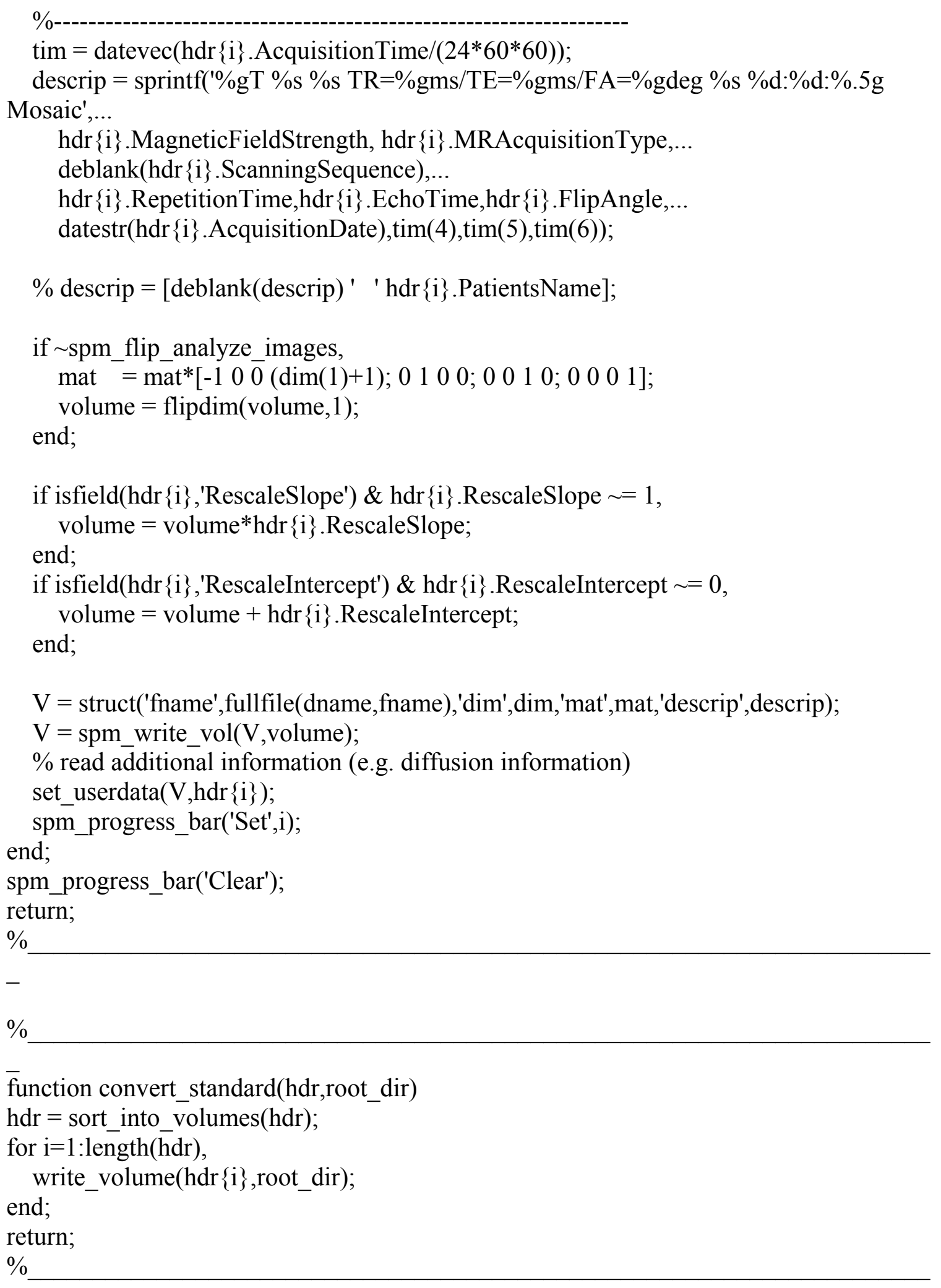




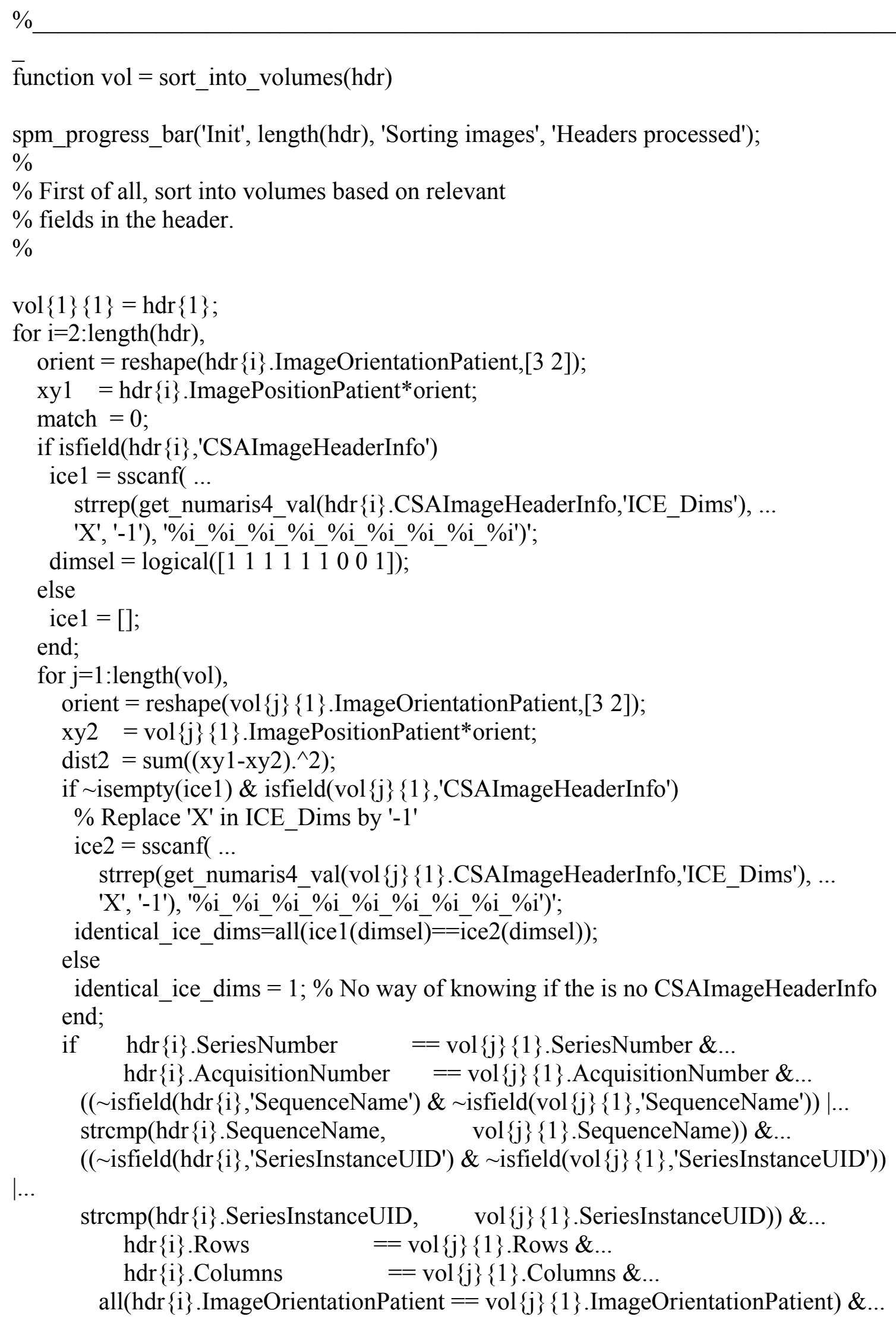




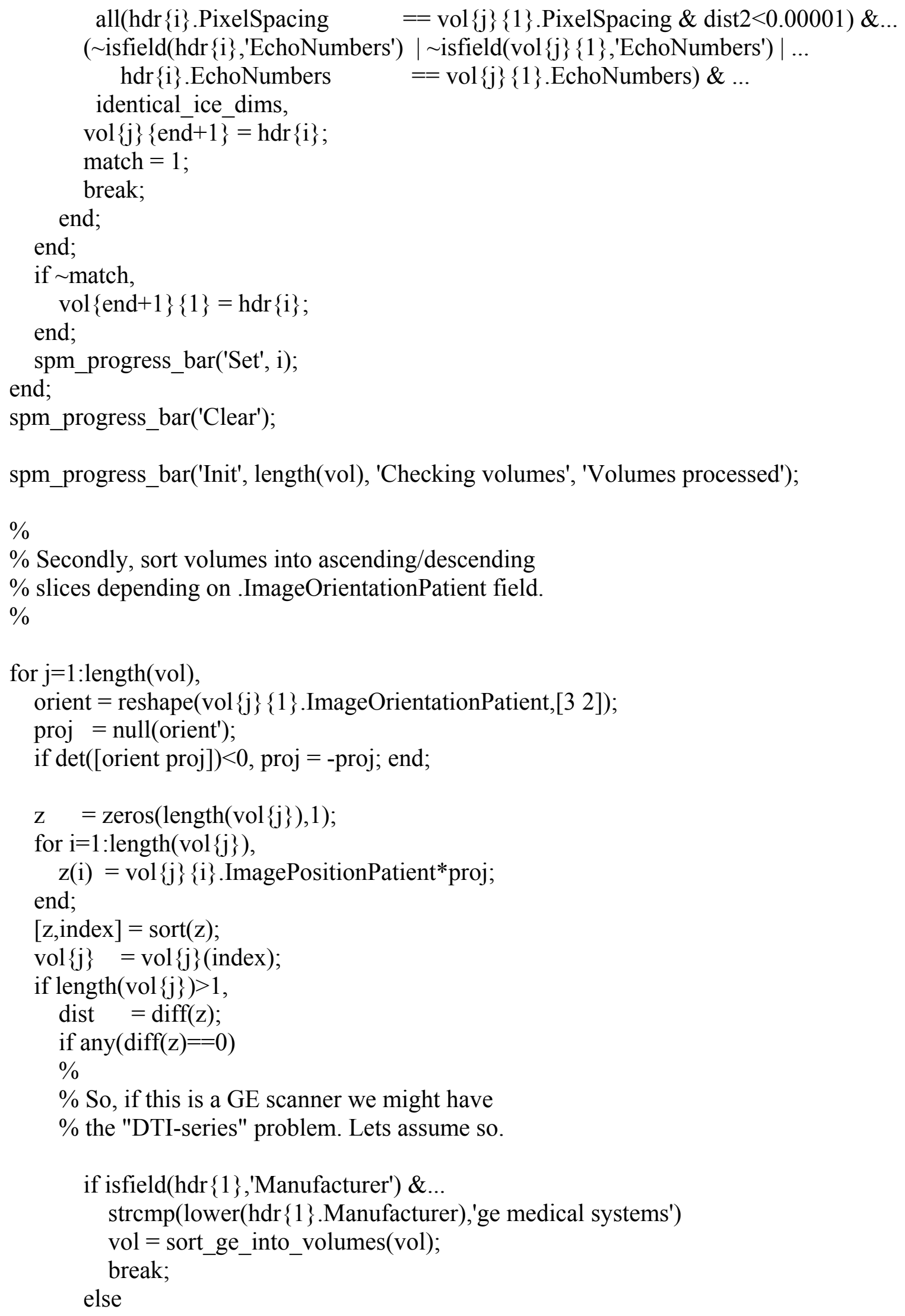


warning('Looks like there is something wrong with the conversion software.'); end

end;

if $\operatorname{sum}\left((\right.$ dist-mean(dist) $\left.) .^{\wedge} 2\right) /$ length(dist $)>0.001$, warning('Variable slice spacing'); end;

end;

spm_progress_bar('Set',j);

end;

spm_progress_bar('Clear');

return;

$\%$

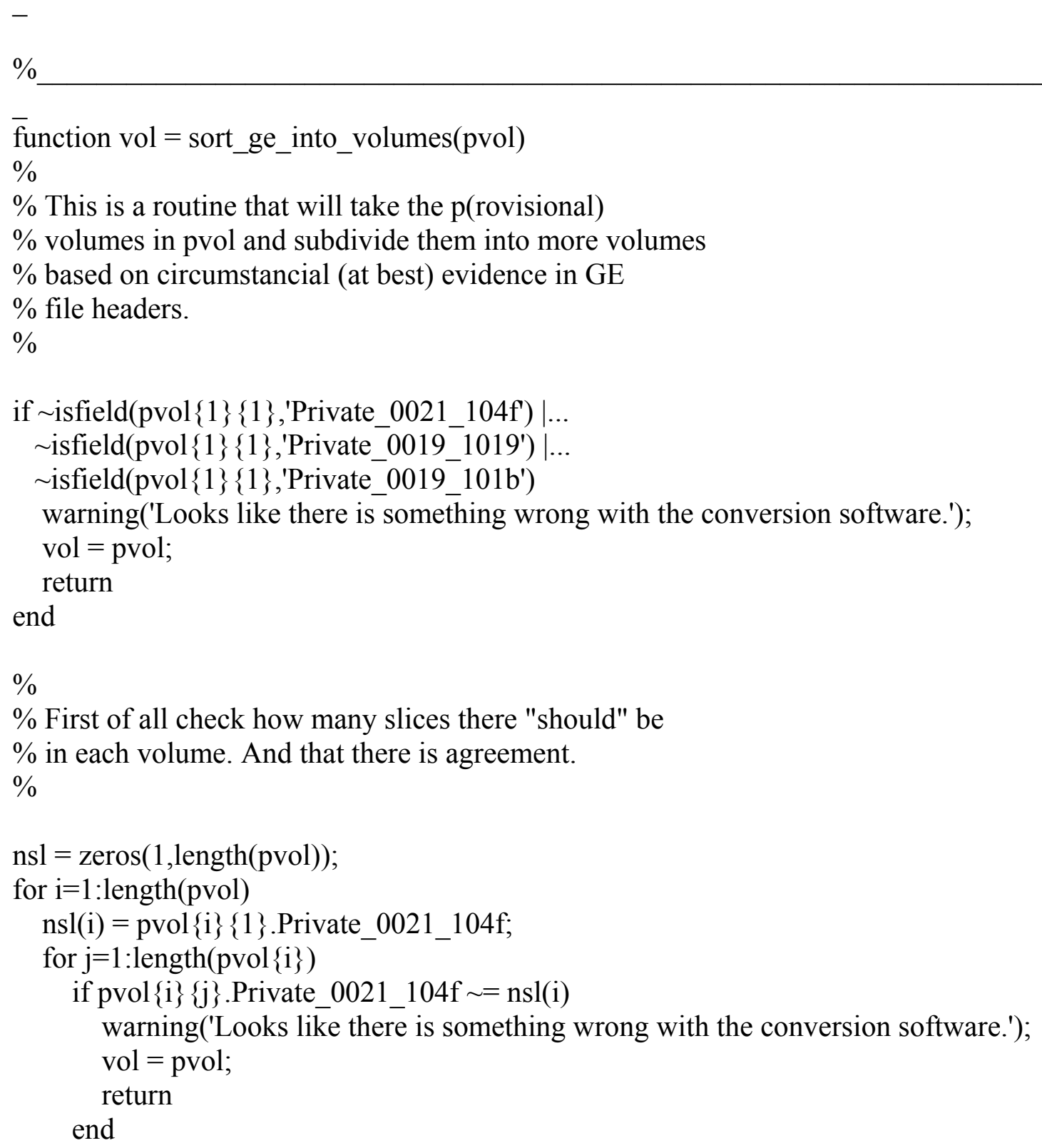




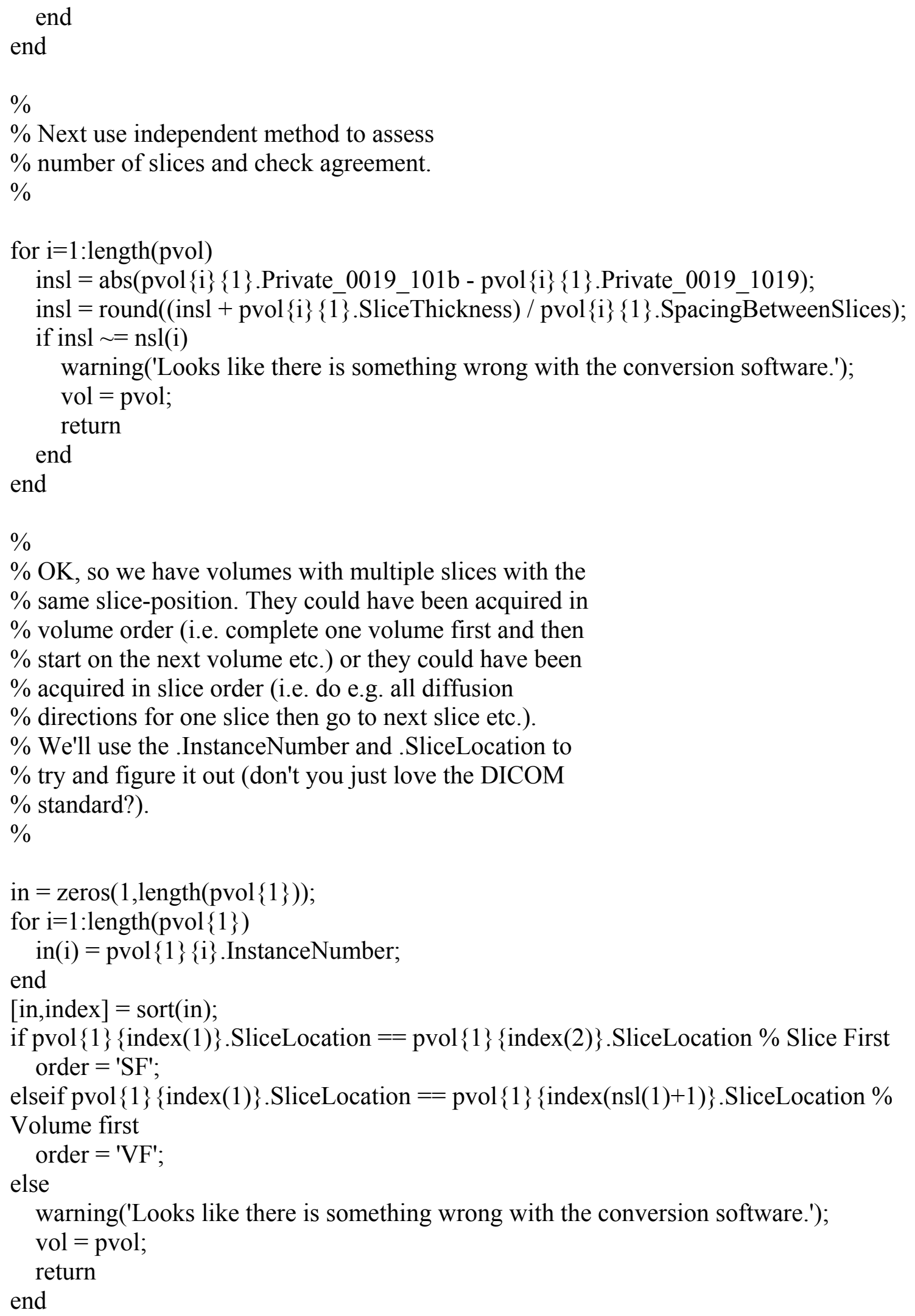




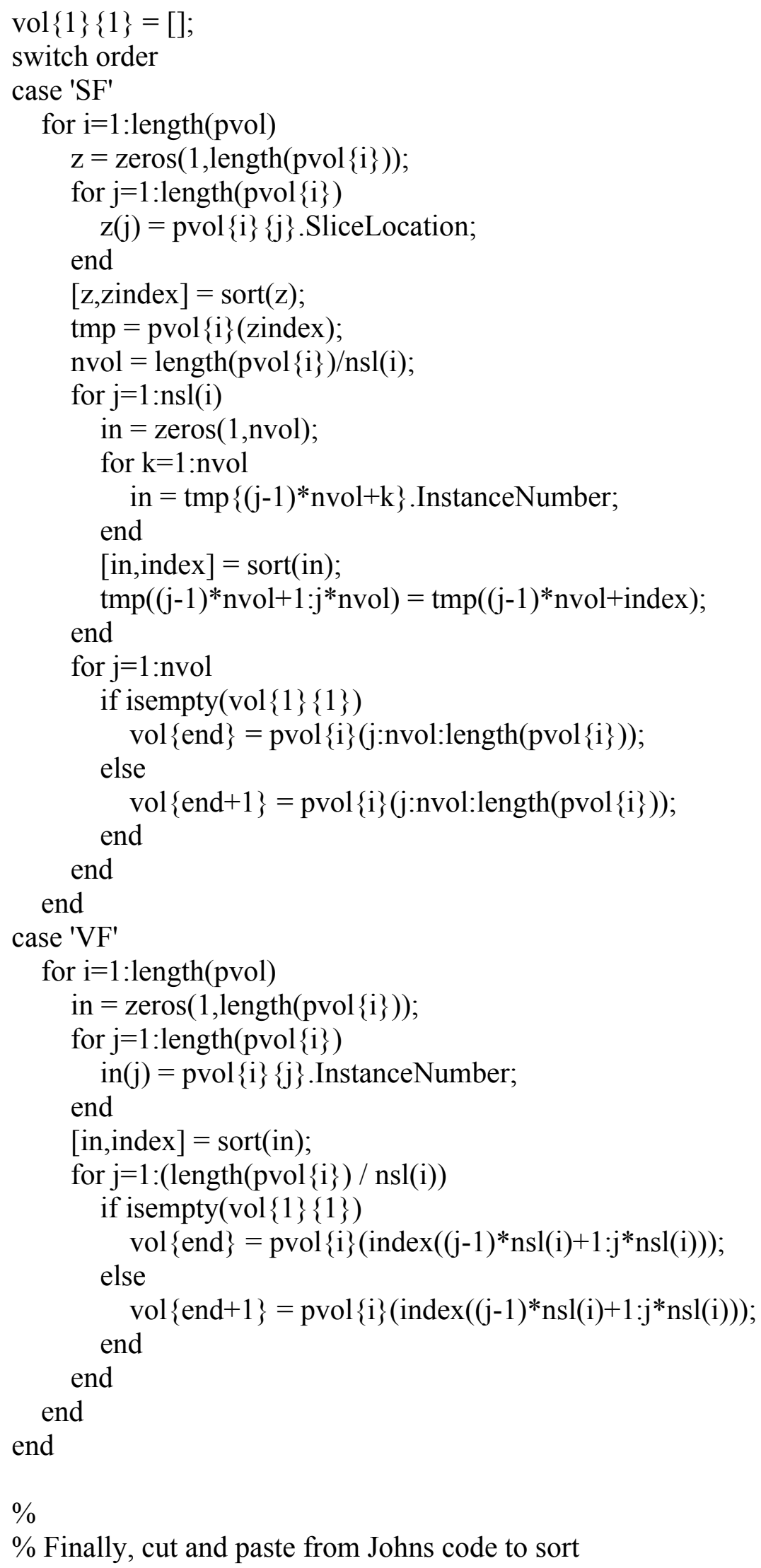

$\%$ Finally, cut and paste from Johns code to sort 


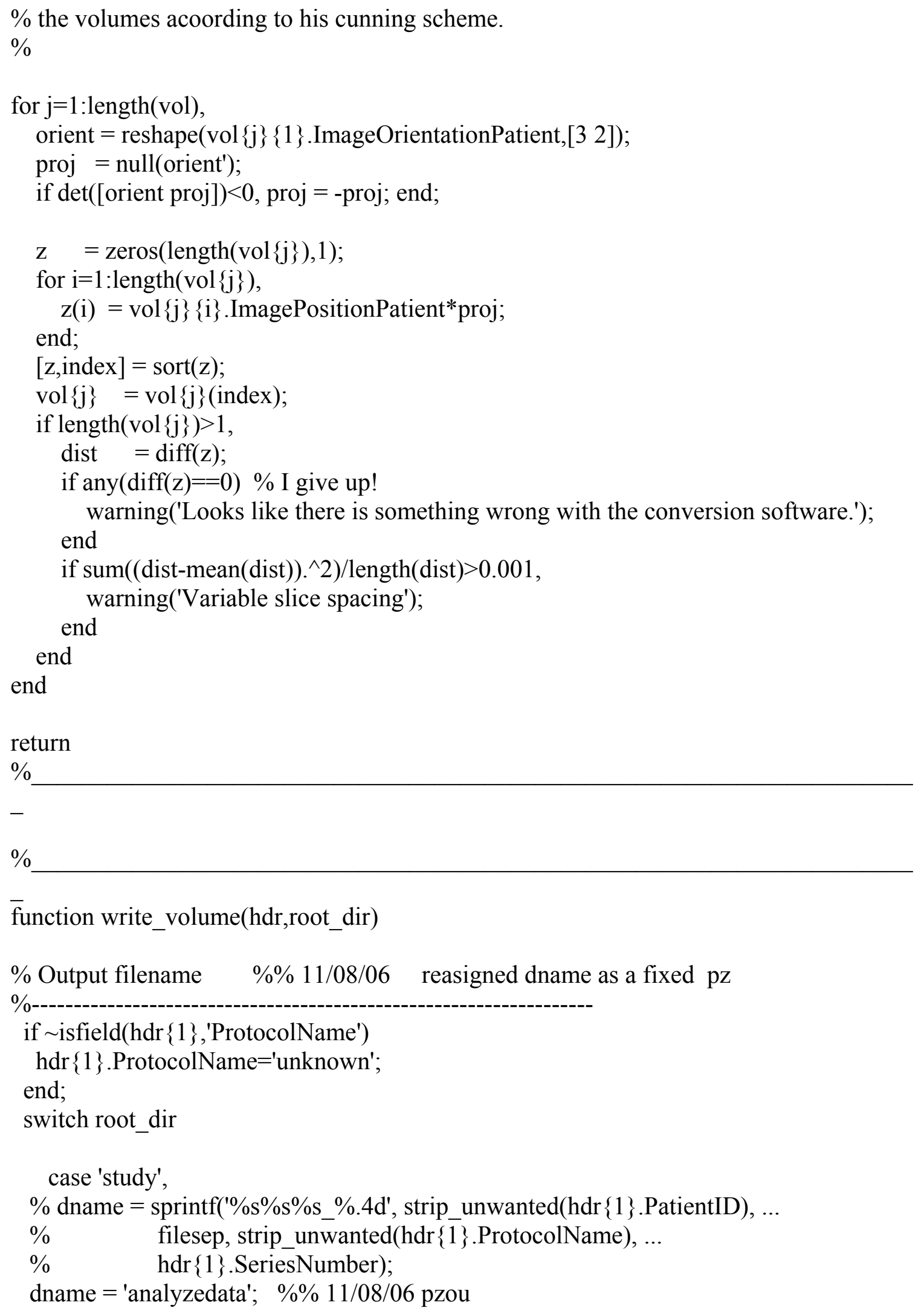




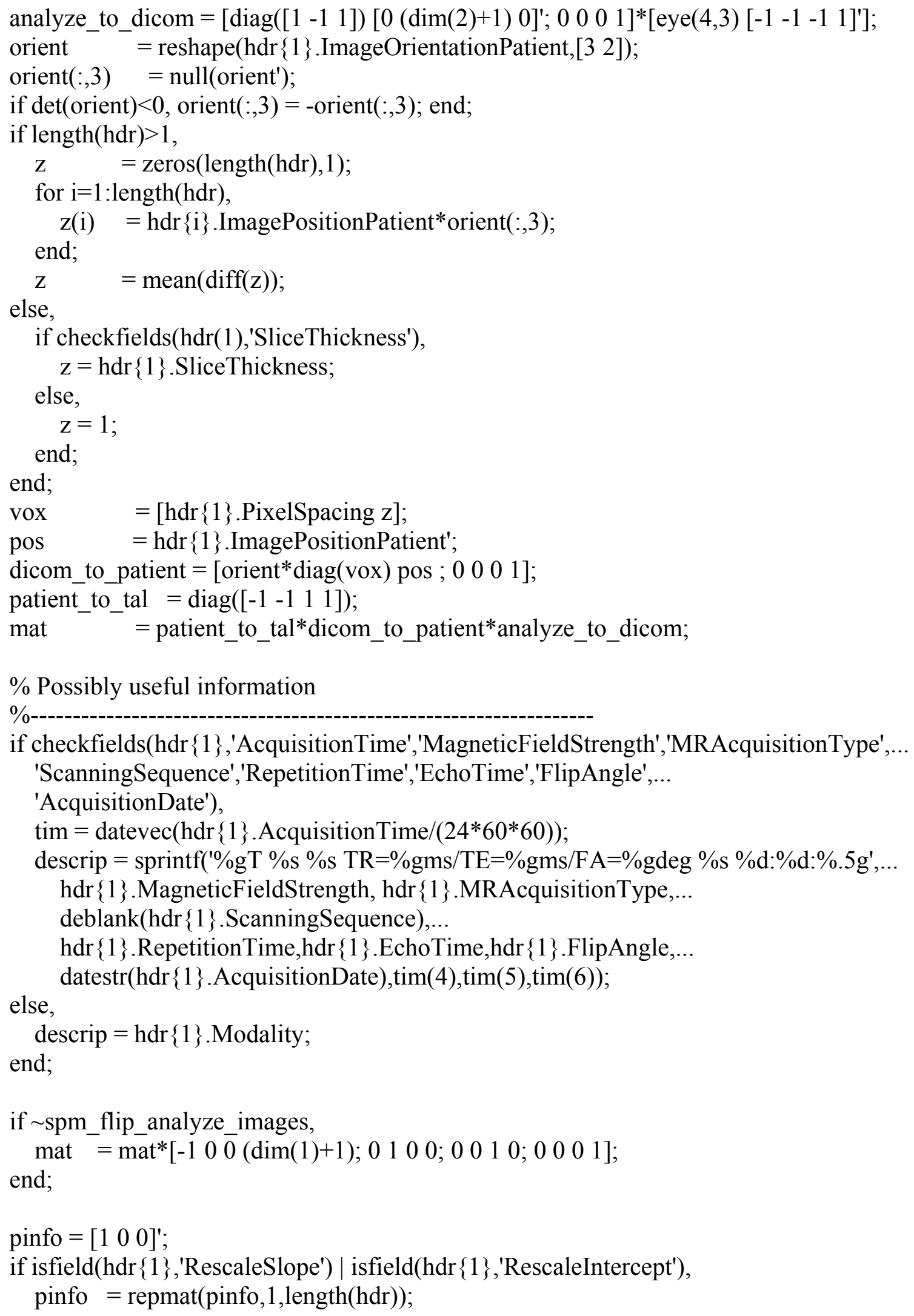




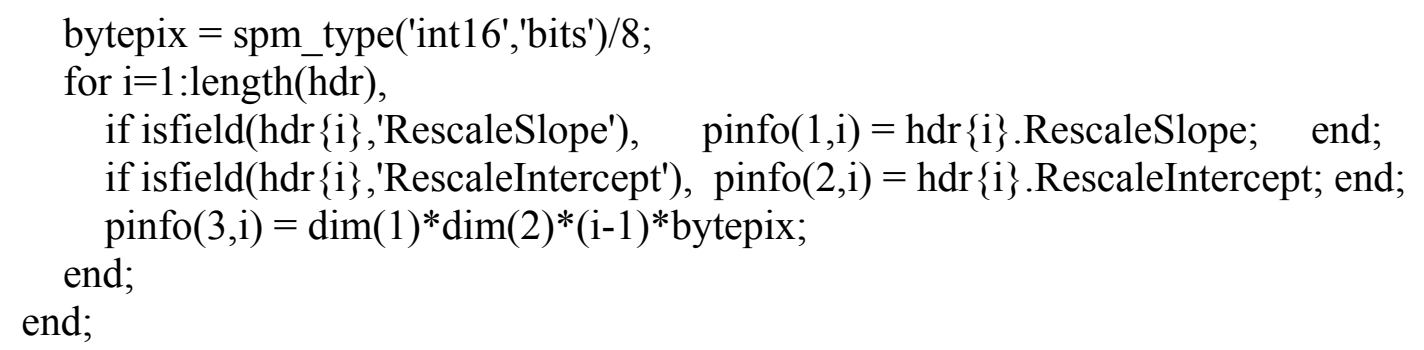




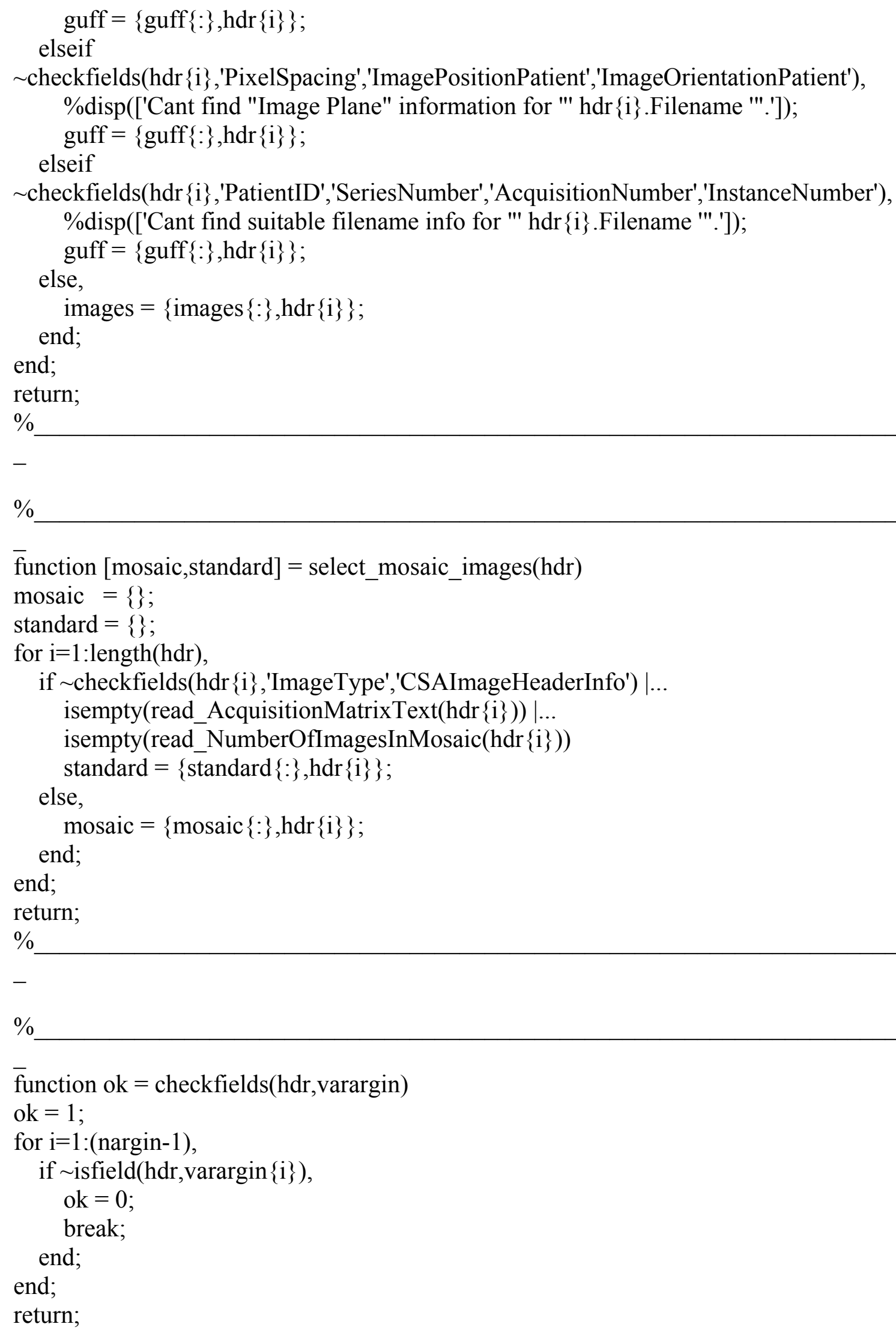




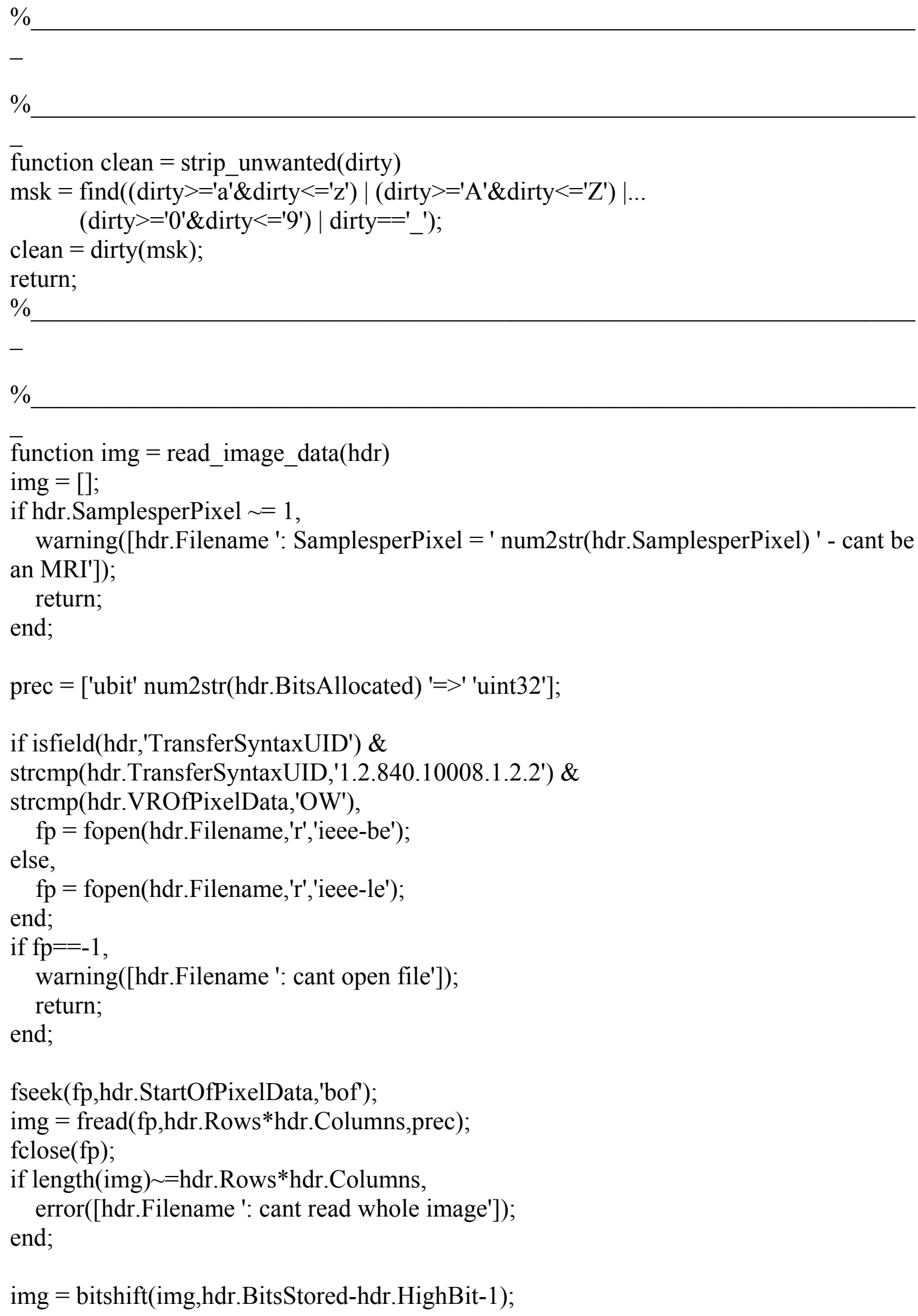




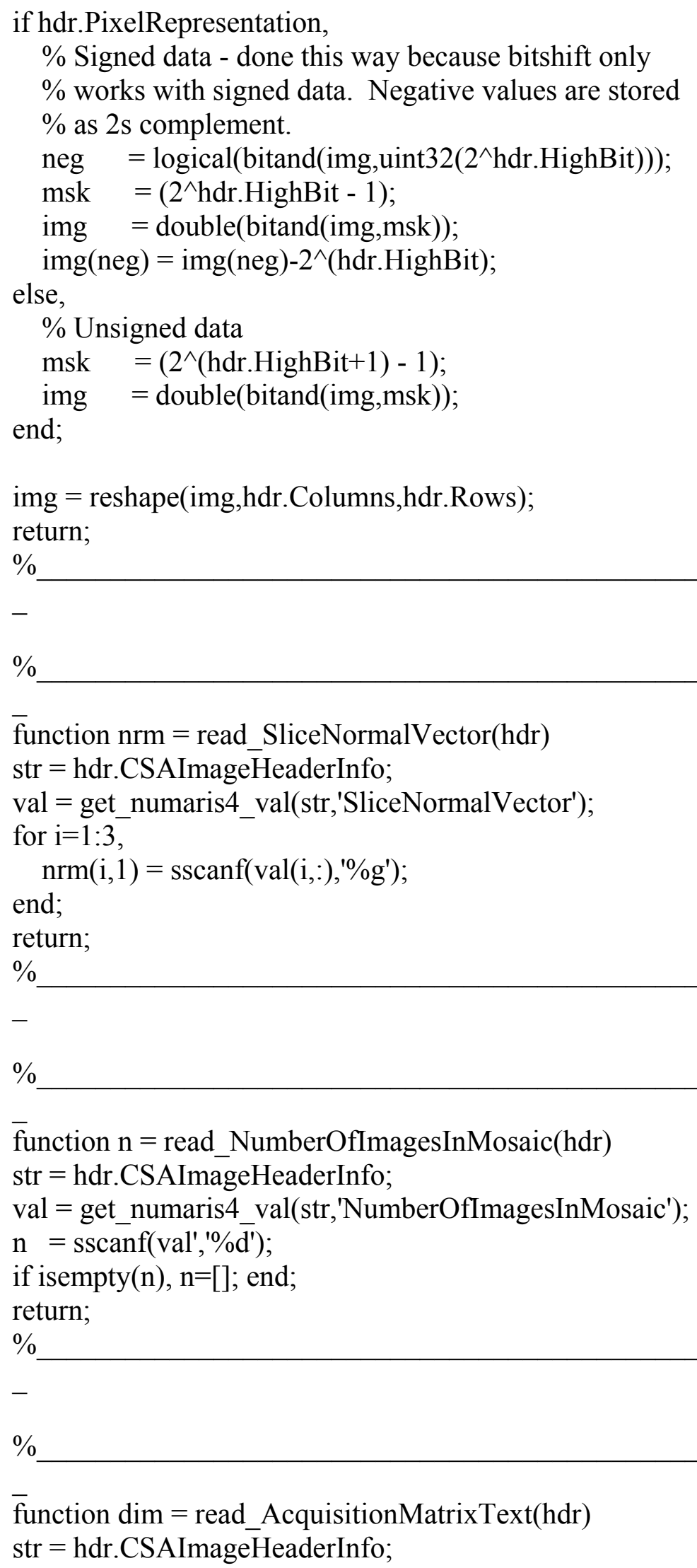




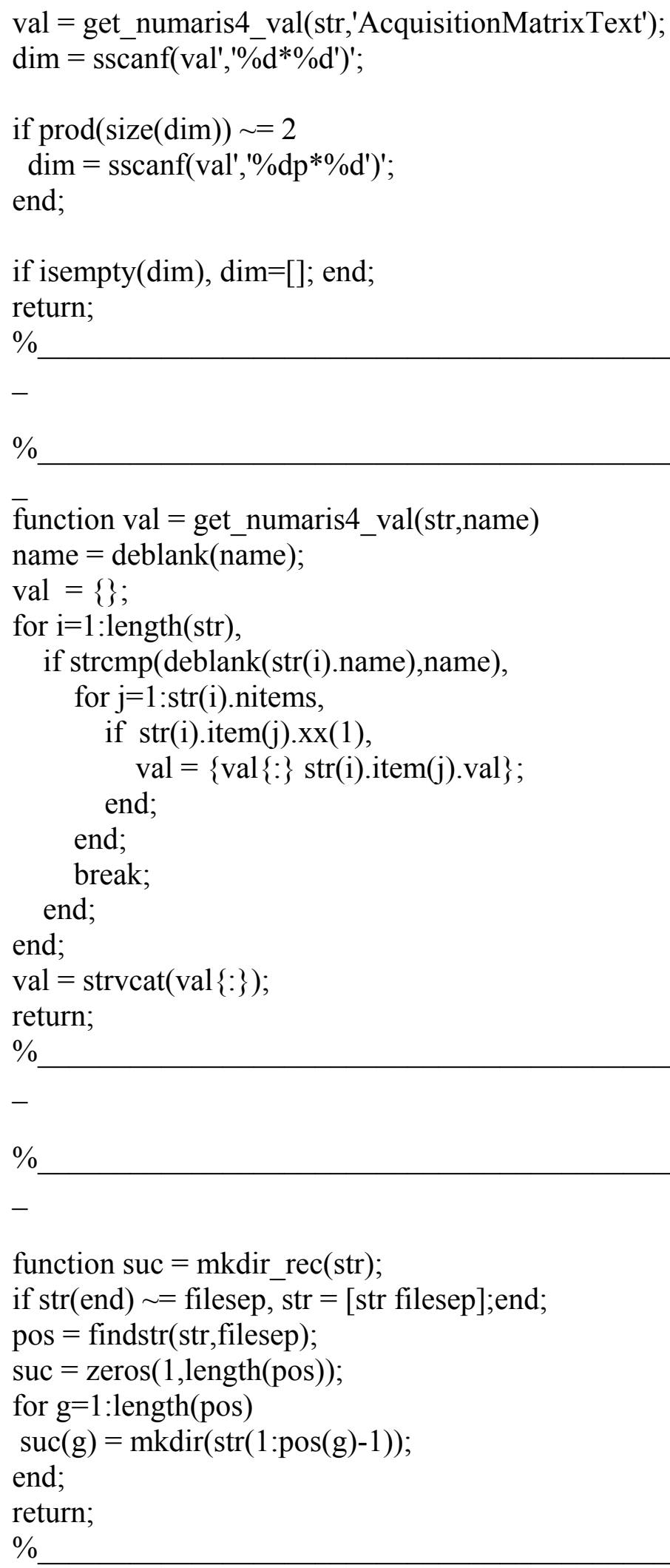




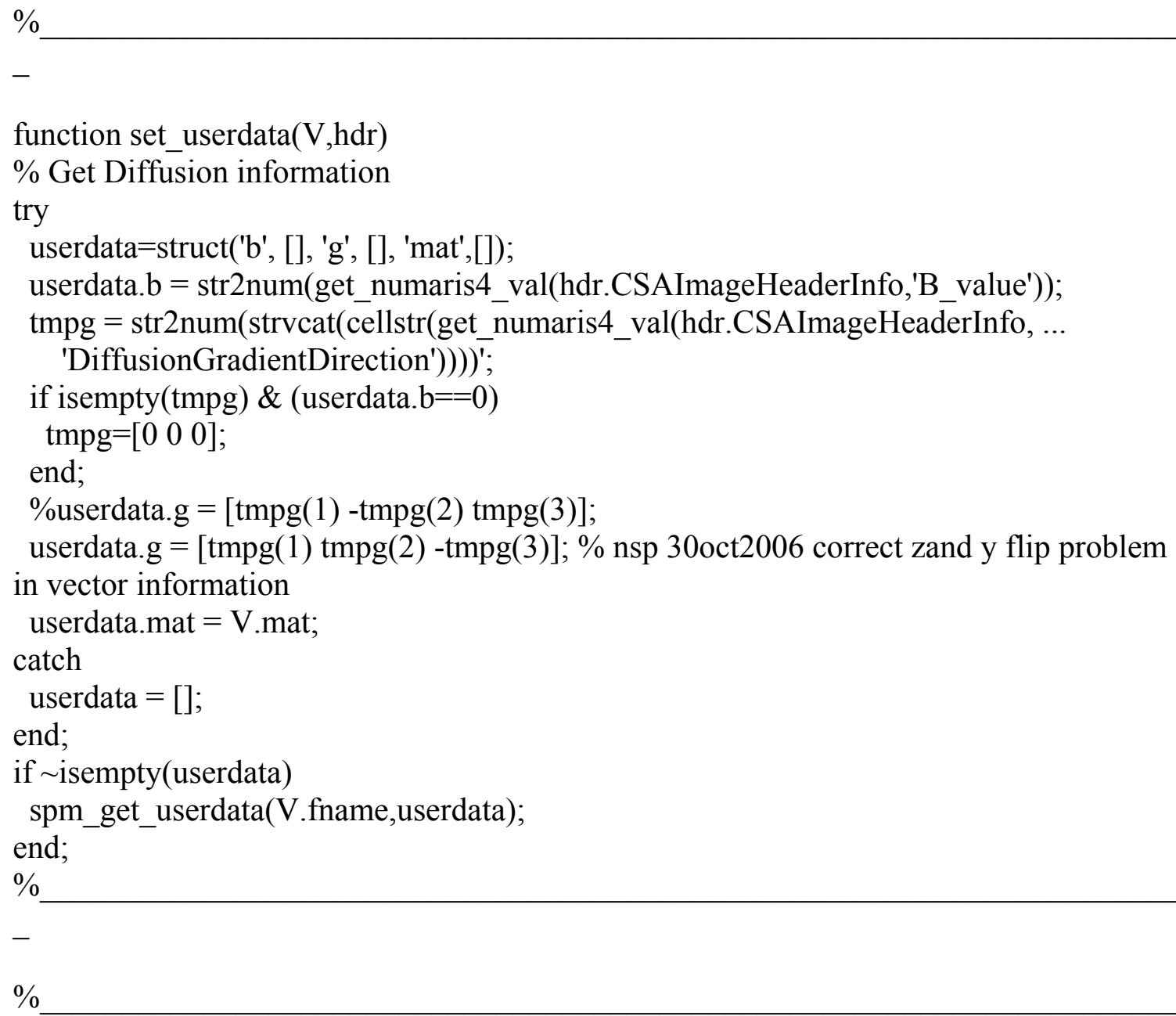




\section{Appendix D. SPM2 Volkmar Diffusion Regression}

function varargout=dti_dt_regress(varargin)

$\%$ Tensor(regression)

$\%$

$\%$ Compute diffusion tensor components from at least 6 diffusion weighted

$\%$ images $+1 \mathrm{~b} 0$ image. Gradient directions and strength are read from the

$\%$ corresponding .mat files. If applicable, normalisation parameters will be

$\%$ taken into account. The resulting B matrix is then given to spm_spm_ui as

$\%$ a Design matrix for a multiple regression analysis.

$\%$

$\%$ If there are repeated measurements or one measurement, but different $b$

$\%$ value levels, then options for non-sphericity correction will be

$\%$ offered. The rationale behind this is the different amount of error

$\%$ variance contained in images with different diffusion weighting.

$\%$ If you want to take advantage of non-spericity correction, you should use

$\%$ an explicit mask to exclude any non-brain voxels from analysis, because

$\%$ the high noise level in non-brain-areas of diffusion weighted images will

$\%$ render the estimation of variance components invalid.

$\%$

$\%$ If there are more than 6 diffusion weighting directions in the data

$\%$ set, tensors of higher order can be estimated. This allows to model

$\%$ diffusivity profiles in voxels with e.g. fibre crossings more

$\%$ accurately than with order- 2 tensors.

$\%$

$\%$ Output images are saved as $\mathrm{D}^{*}$ basename.img, where basename is derived

$\%$ from the original filenames. Directionality components are encoded by

$\%$ ' $x$ ', 'y', 'z' letters in the filename.

$\%$

$\%$ References:

$\%$ The algorithm used to determine the design matrix for SPM2 stats in this

$\%$ routine is based on

$\%$ [BML94] Basser, Peter J, James Mattiello and Denis LeBihan. Estimation

$\% \quad$ of the Effective Self-Diffusion Tensor from the NMR Spin Echo.

$\% \quad$ Journal of Magnetic Resonance, B(103):247--254, 1994.

$\%$ and is developed for the general order-n tensors in

\% [ÖM03] Evren Örszalan, Thomas H. Mareci. Generalized Diffusion Tensor

$\% \quad$ Imaging and Analytical Relationships Between Diffusion Tensor

$\% \quad$ Imaging and High Angular Resolution Diffusion Imaging. Magnetic

$\% \quad$ Resonance in Medicine, 50:955 -- 965, 2003.

$\%$ although the direction scheme actually used depends on the applied

$\%$ diffusion imaging sequence.

$\%$

$\%$ Batch processing

$\%$ FORMAT [bchdefaults sessiondefaults] = dti_dt_regress('defaults') 


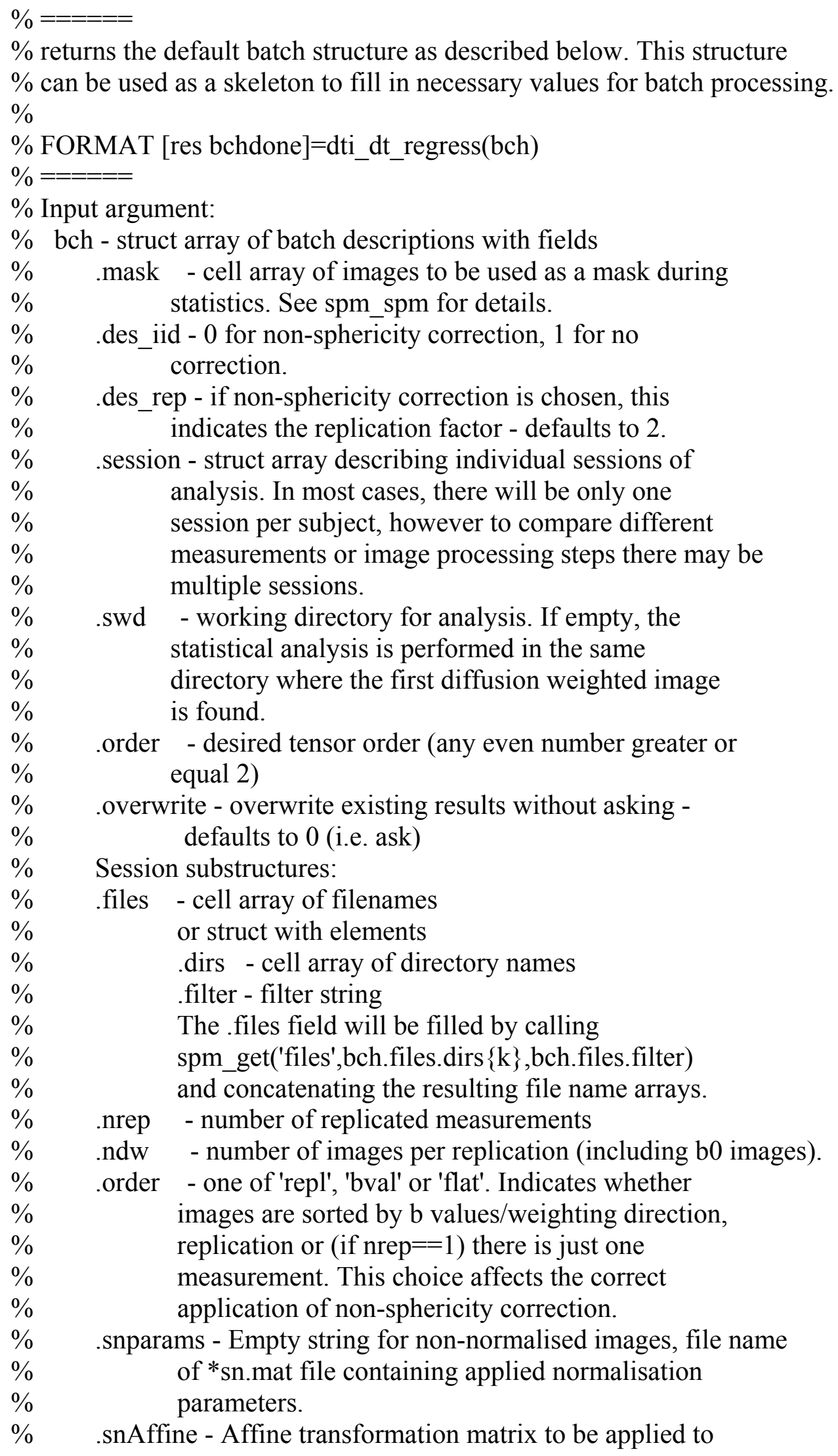




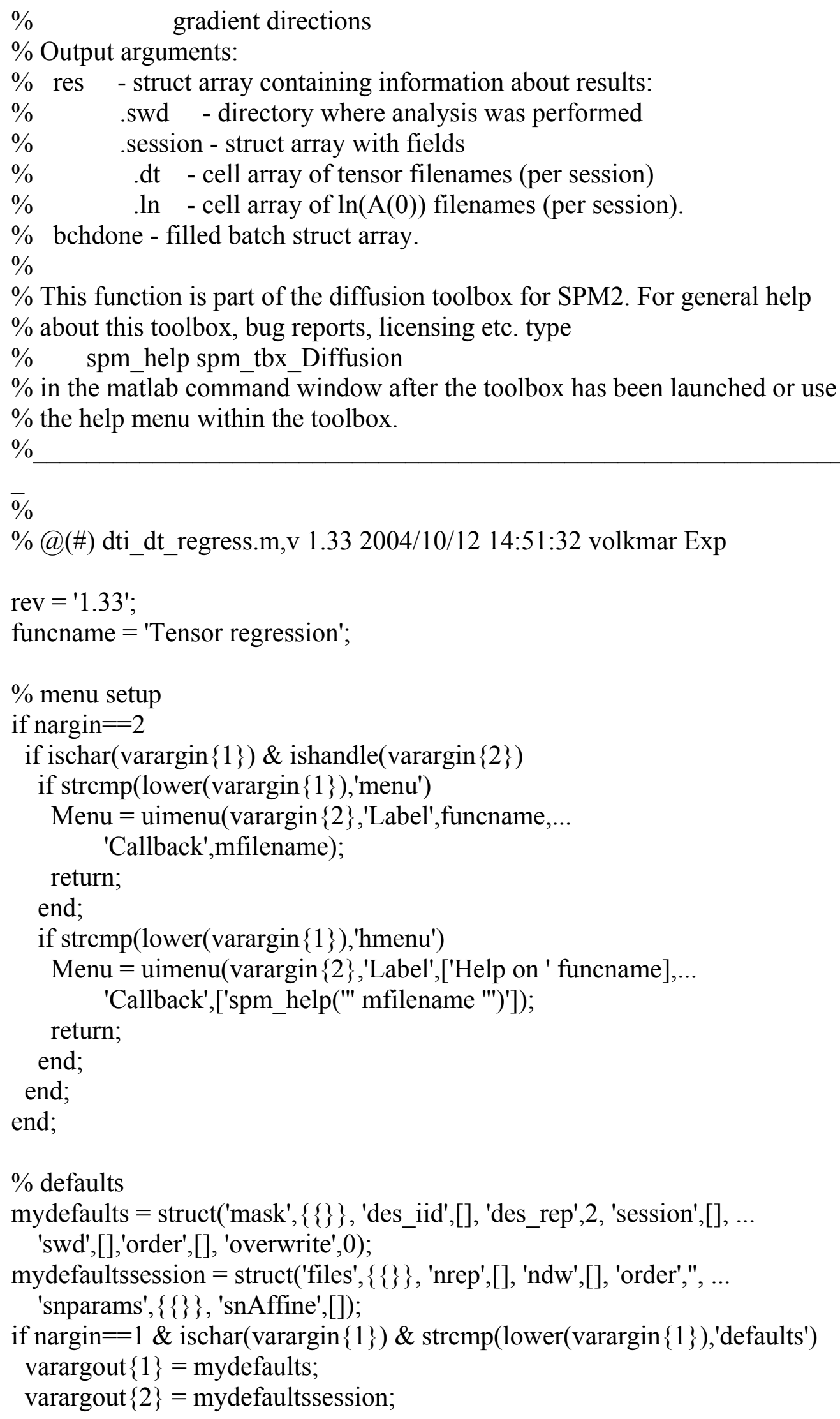


return;

end;

$\%$ function preliminaries

Finter=spm_figure('GetWin','Interactive');

spm_input('!DeleteInputObj');

Finter=spm('FigName',funcname,Finter);

SPMid = spm('FnBanner',mfilename,rev);

$\%$ function code starts here

$\%$ Check input arguments

if (nargin==1) \& isstruct(varargin $\{1\})$

$\%$ Check for spmr_pp-batch

if isfield(varargin $\{1\}$,'step')

bch $=$ checkfields(mydefaults, varargin $\{1\}$.step);

else

bch $=$ checkfields (mydefaults, varargin $\{1\})$;

end;

nana $=\operatorname{numel}(\mathrm{bch})$;

else

nana $=$ spm input('\# analyses','!+1','e',"');

bch=repmat(mydefaults,[nana 1]);

end;

for $\mathrm{ca}=1$ :nana

spm_input(sprintf('Analysis \%d', ca),1,'d',funcname);

if isempty(bch(ca).order)

bch(ca).order $=$ spm_input('Which tensor order?', '!+1', 'be1', ...

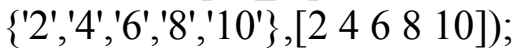

end;

if isempty(bch(ca).mask)

bch(ca).mask $=$ spm_get $\left(I n f,{ }^{*}\right.$ IMAGE', ..

\{sprintf('Analysis \% $\mathrm{d}$ - mask images (optional)',... ca) $\}$ );

if isempty(bch(ca).mask) \% no mask supplied

bch(ca).mask $=\{"\}$;

end;

end,

def_iid=isempty(bch(ca).mask $\{1\})+1$;

if isempty(bch(ca).des_iid)

bch(ca).des_iid = spm_input('non-sphericity correction?', '!+1', 'y/n', ...

end; [0,1],def_iid); 


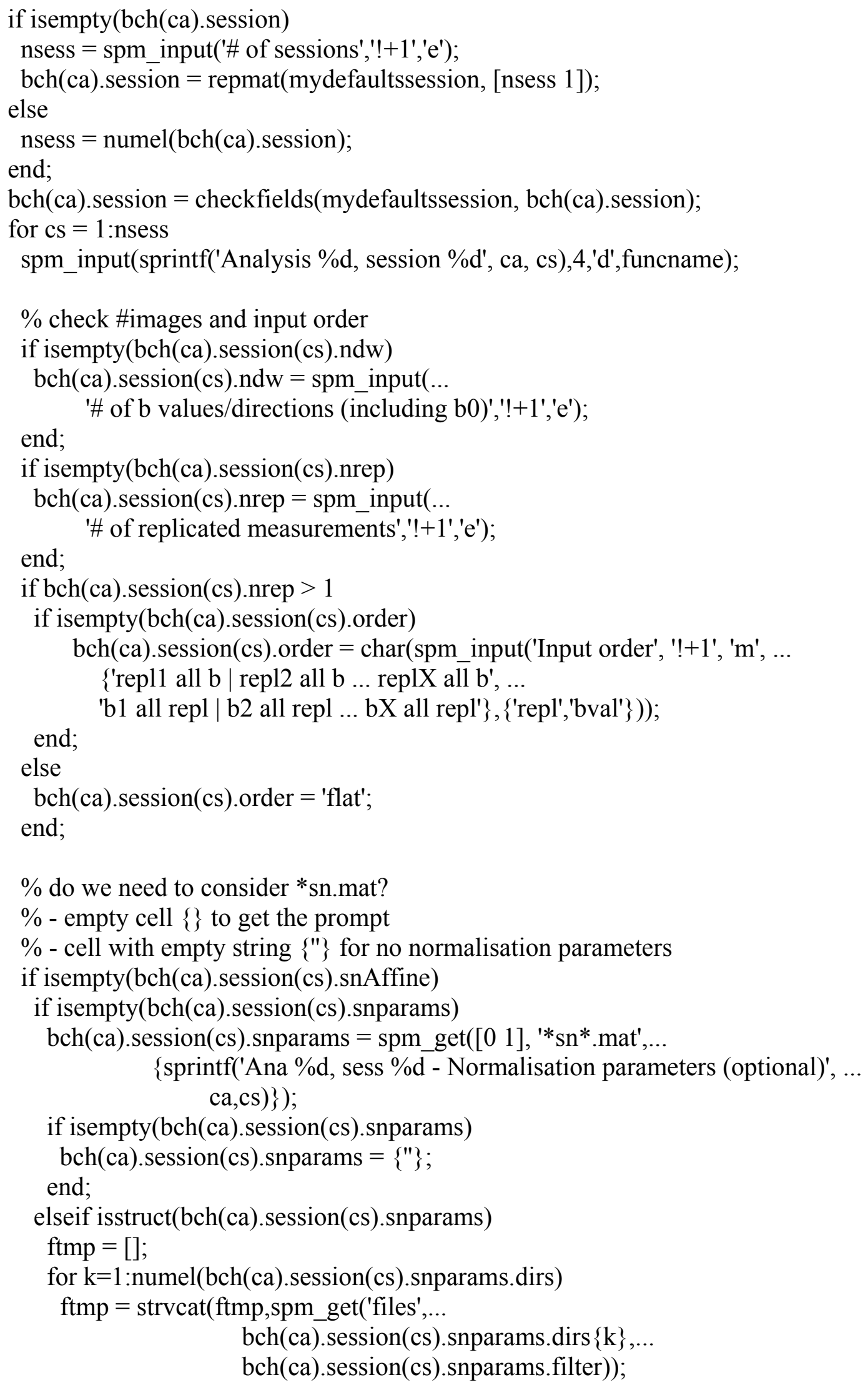


end;

bch(ca).session(cs).snparams = cellstr(ftmp);

end;

$[\mathrm{bch}(\mathrm{ca}) \cdot \operatorname{session}(\mathrm{cs}) \cdot \operatorname{snAffine} \mathrm{pref}]=\ldots$

dti_get_snaffine(bch(ca).session(cs).snparams $\{1\})$;

end;

$\%$ get images

if isempty(bch(ca).session(cs).files)

bch(ca).session(cs).files $=$ spm_get(bch(ca).session(cs).nrep*...

bch(ca).session(cs).ndw, [pref '*IMAGE'], ...

$\{$ sprintf('Ana \%d, sess \%d - All images',ca,cs)\});

elseif isstruct(bch(ca).session(cs).files)

$\mathrm{ftmp}=[]$;

for $\mathrm{k}=1$ :numel(bch(ca).session(cs).files.dirs)

$\mathrm{ftmp}=\operatorname{strvcat}\left(\mathrm{ftmp}, \mathrm{spm} \_\right.$get('files',... bch(ca).session(cs).files.dirs $\{k\}, \ldots$

bch(ca).session(cs).files.filter));

end;

bch(ca).session(cs).files $=$ cellstr(ftmp);

end;

end;

end;

$\%$ save working directory

cwd=pwd;

$\%$ get design definition

$\mathrm{D}=$ spm_spm_ui('DesDefs_Stats'); \% Get design definitions

ind $=$ find $(\operatorname{strcmp}($ lower(strvcat(D.DesName) $),\{$ 'multiple regression' $\}))$;

if isempty(ind)

error('Design definition for multiple regression analysis not found');

end;

$\mathrm{D}=\mathrm{D}$ (ind); \% Use Multiple regression as template

for $\mathrm{ca}=1$ :nana

$\%$ get beta filename stub \& new working dir

if isempty(bch(ca).swd)

[res(ca).swd betafname e v] = fileparts(bch(ca).session(1).files $\{1\})$;

betafname $=[$ '_' betafname $]$;

if isempty(res(ca).swd)

$\operatorname{res}(\mathrm{ca}) . \mathrm{swd}=\mathrm{pwd}$;

end;

else

betafname $=\operatorname{sprintf('\_ ana\% d',ca);}$

res(ca).swd = bch(ca).swd;

end; 


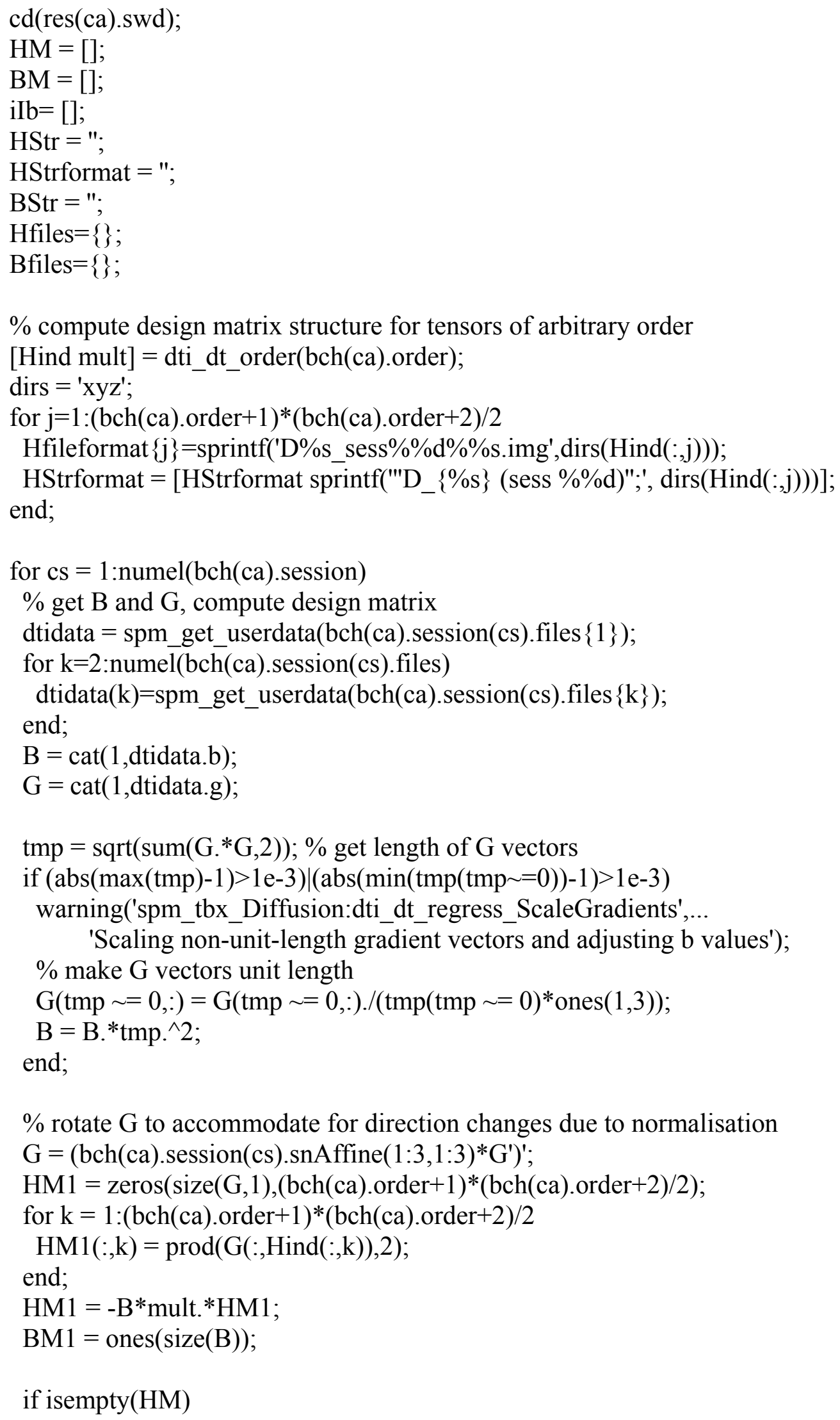




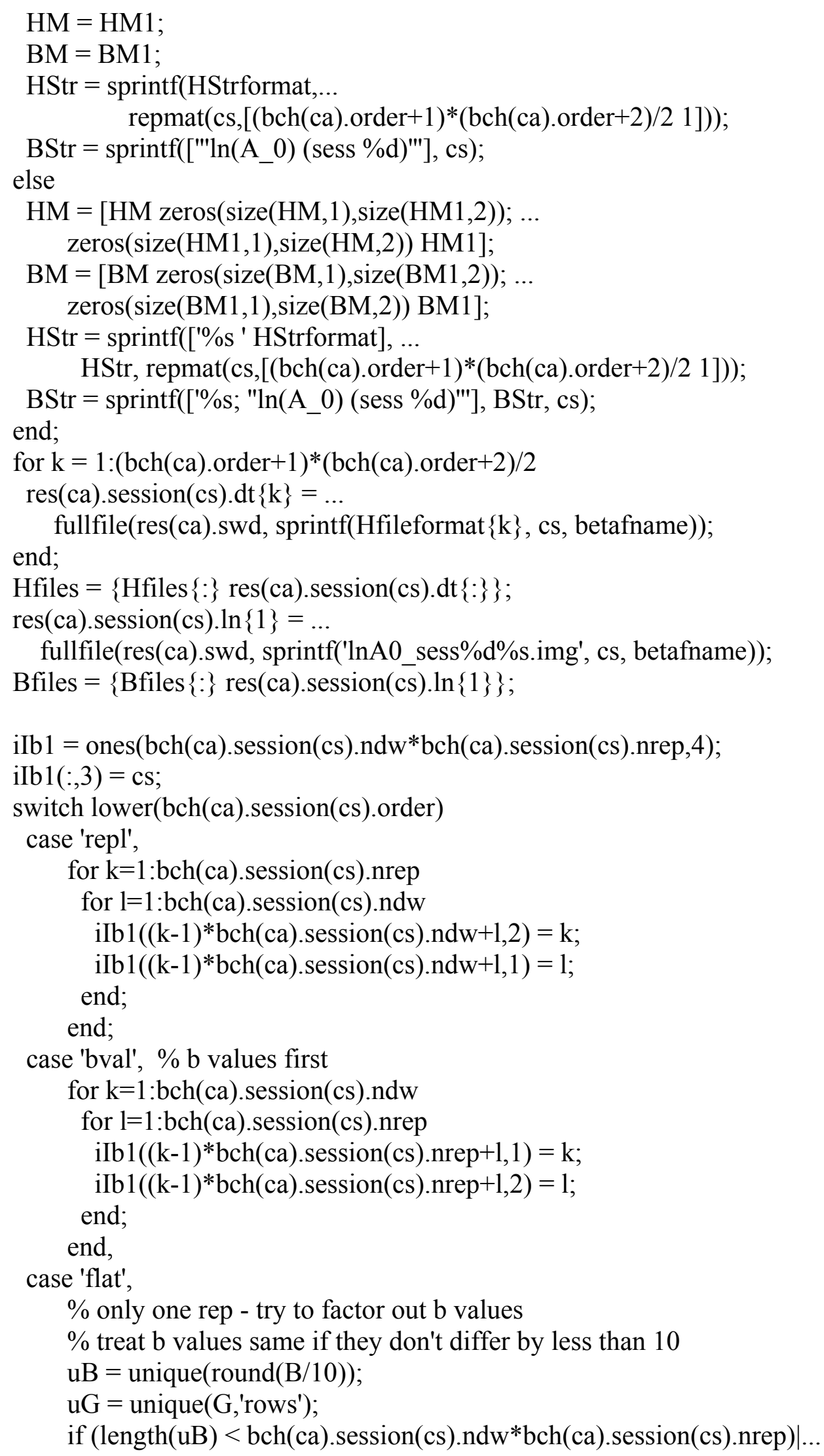




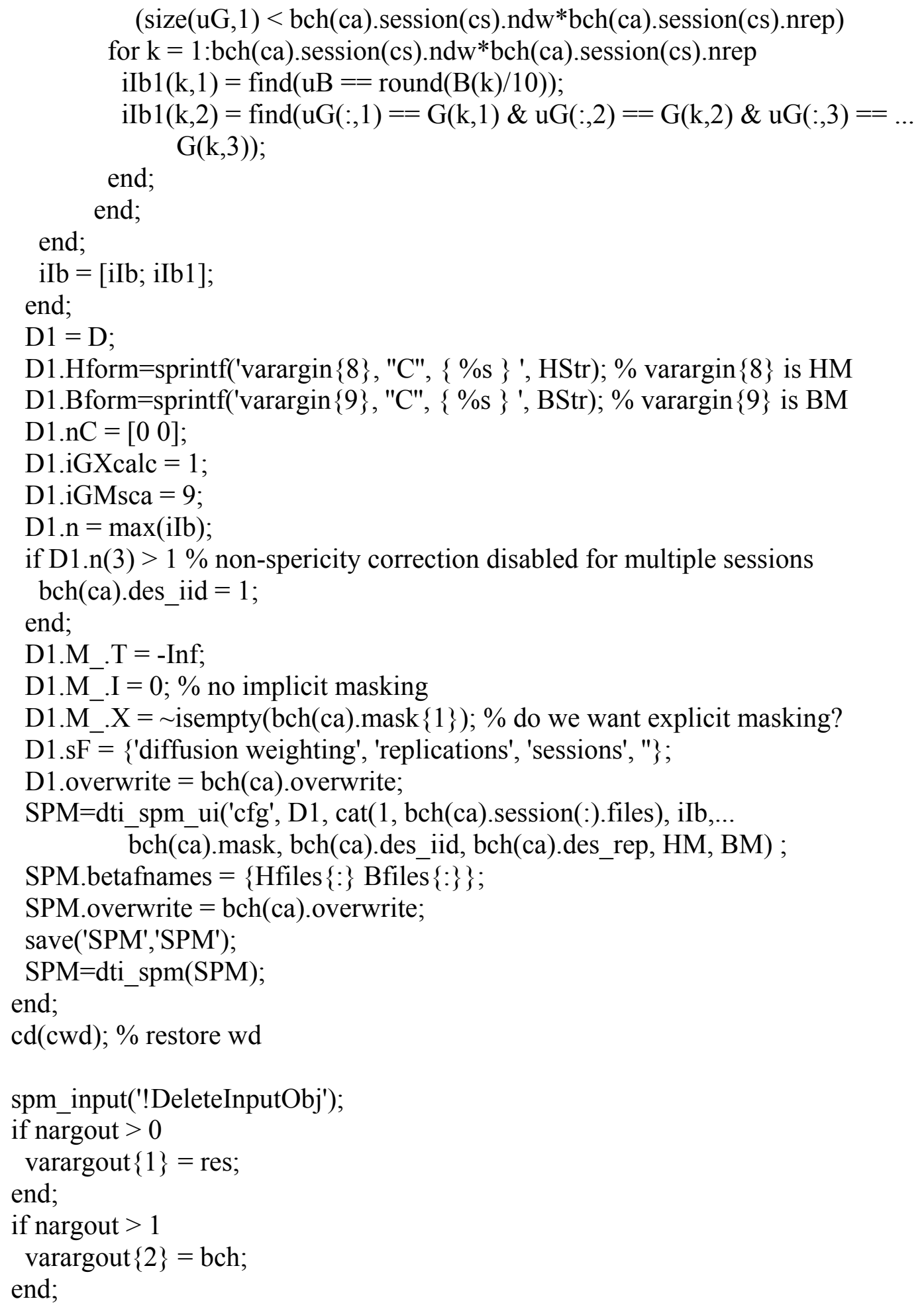




\section{Appendix E. TBSS Preprocessing Batch Files for Parallel Processing}

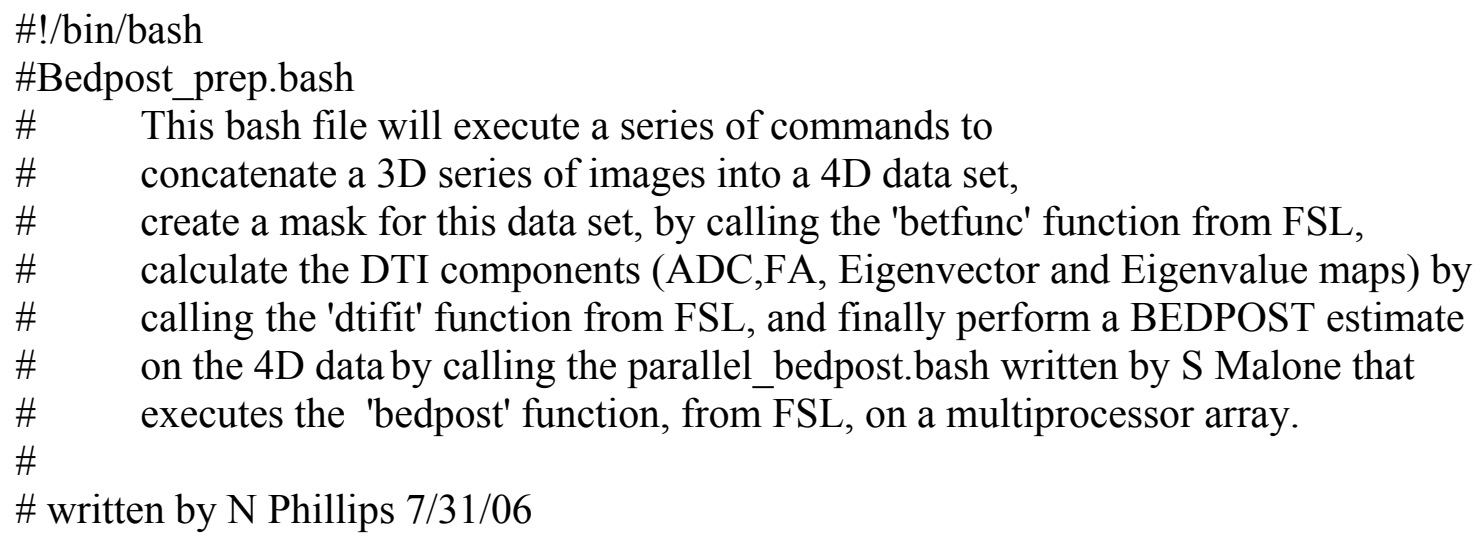

\#Copy template 4D header and rename data.hdr cp \$baseDir/bvecsx4 \$myPath/bvecs

cp \$baseDir/bvalsx4 \$myPath/bvals

done $\}<$ \$baseDir/DTI-copy-list

$\operatorname{extract}()\{$

\# call BET function from FSl for each data set 
while read patID examDate ser1; do

$$
\begin{aligned}
& \text { export myPath="\$baseDir/\$patID/\$examDate/"analyzedata"" } \\
& \text { cd \$myPath } \\
& \text { \#\# brain extraction tool } \\
& \text { \# } \quad \text {-f,--data } \quad \text { fractional intesity threshold }<0-1>\text { default }=0.5 \\
& \text { \# } \quad-\mathrm{m}, \quad \text { generate binary mask } \\
& \text { \# } \quad-\mathrm{v}, \quad \text { verbose text output }
\end{aligned}
$$

betfunc \$myPath/nodif \$myPath/nodif_brain -m -f 0.25

done $\}<$ \$baseDir/DTI-copy-list

DTIfit() \{

\# call DTIfit function from FSL for each data set

while read patID examDate ser 1 ; do

export myPath="\$baseDir/\$patID/\$examDate/"analyzedata""

cd \$myPath

\#\# Compulsory arguments (You MUST set one or more of):

\# $\quad$-k,--data dti data file

\# -o,--out Output basename

\# -m,--mask Bet binary mask file

\# -r,--bvecs b vectors file

\# -b,--bvals b values file

dtifit -k \$myPath/data.nii.gz -o \$myPath/dti -m \$myPath/nodif_brain_mask.nii.gz -r \$myPath/bvecs -b \$myPath/bvals

done $\}<$ \$baseDir/DTI-copy-list

Bedpost() \{

\#call Bedpost function from FSl for each data set

while read patID examDate ser 1 ; do

export myPath="\$baseDir/\$patID/\$examDate/"analyzedata"" cd \$myPath 
\#call for queue on Hartwell

\#bsub -q fsl -n 32 ./parallel_bedpost.bash \$myPath

\#command to run on Gonzalez

Bedpost \$myPath

done $\}<$ \$baseDir/DTI-copy-list

catimg

extract

DTIfit

Bedpost

exit 


\section{Vita}

Nicholas Phillips was born in Columbus Ohio in 1967. He has worked as a roofer, painter, dish washer, x-ray transporter, unit secretary, phlebotomist and medical assistant (with limited x-ray licensure). He also served as a cavalry scout in the US Army and Tennessee Army National Guard for 10 years of combined service. He graduated from Pellissippi State Technical Community College in 1998 with an AS in general science. Because of his academic achievement at Pellissippi State, he was awarded a scholarship at Maryville College, where he completed his BA in Biochemistry in 2000. He then entered Medical School at the University of Tennessee Health Science Center where he completed his MD and PhD in Biomedical Engineering and Imaging in 2009. He was honored during graduation by receiving the 'Outstanding Student Award' in Neurology and the American Acadamy of Neurology medical student prize for excellence in neurology. He subsequently plans to begin training in a combined research residency program at the University of Tennessee Health Science Center and St. Jude Childrens Research Hosptial in Pediatric Neurology. 